\title{
Low-Cost Multi-Modal Wireless Sensor Platform for Smart Buildings
}

\section{Approved for public release.} Distribution is unlimited.
Teja Kuruganti

Stephen Killough Mohammed Olama Pooran Joshi Christopher Winstead Steve Fulton Dave English Christopher Ray $04 / 27 / 2018$ 


\section{DOCUMENT AVAILABILITY}

Reports produced after January 1, 1996, are generally available free via US Department of Energy (DOE) SciTech Connect.

Website www.osti.gov

Reports produced before January 1, 1996, may be purchased by members of the public from the following source:

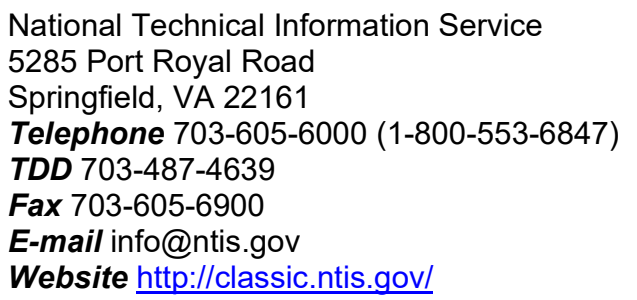

Reports are available to DOE employees, DOE contractors, Energy Technology Data Exchange representatives, and International Nuclear Information System representatives from the following source:

Office of Scientific and Technical Information

PO Box 62

Oak Ridge, TN 37831

Telephone 865-576-8401

Fax 865-576-5728

E-mail reports@osti.gov

Website http://www.osti.gov/contact.html

This report was prepared as an account of work sponsored by an agency of the United States Government. Neither the United States Government nor any agency thereof, nor any of their employees, makes any warranty, express or implied, or assumes any legal liability or responsibility for the accuracy, completeness, or usefulness of any information, apparatus, product, or process disclosed, or represents that its use would not infringe privately owned rights. Reference herein to any specific commercial product, process, or service by trade name, trademark, manufacturer, or otherwise, does not necessarily constitute or imply its endorsement, recommendation, or favoring by the United States Government or any agency thereof. The views and opinions of authors expressed herein do not necessarily state or reflect those of the United States Government or any agency thereof. 
ORNL/TM-2018/934

CRADA/NFE-14-05348

Computational Sciences and Engineering Division

\title{
LOW-COST MULTI-MODAL WIRELESS SENSOR PLATFORM FOR SMART BUILDINGS
}

\author{
Teja Kuruganti \\ Stephen Killough \\ Mohammed Olama \\ Pooran Joshi \\ Christopher Winstead \\ Steve Fulton \\ Dave English \\ Christopher Ray
}

Date Published: April 2018

\author{
Prepared by \\ OAK RIDGE NATIONAL LABORATORY \\ Oak Ridge, TN 37831-6283 \\ managed by \\ UT-BATTELLE, LLC \\ for the \\ US DEPARTMENT OF ENERGY \\ under contract DE-AC05-00OR22725
}




\section{CONTENTS}

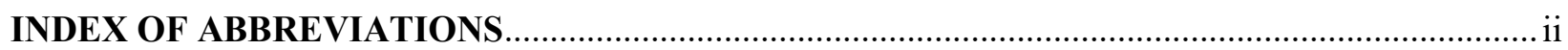

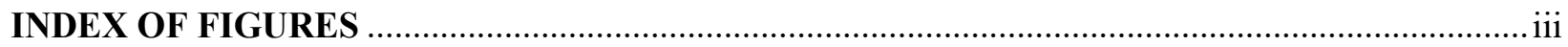

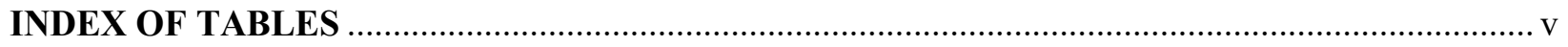

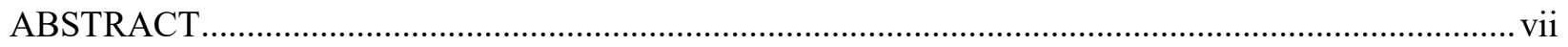

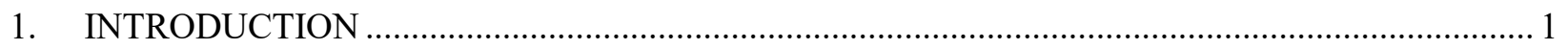

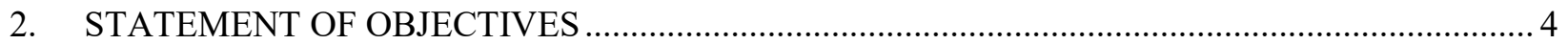

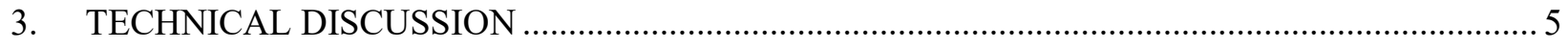

3.1 MATERIALS DEVELOPMENT AND PROCESSING ……............................................. 5

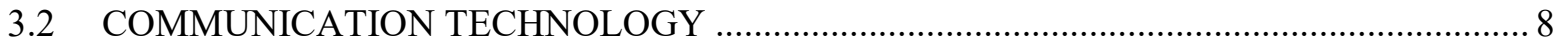

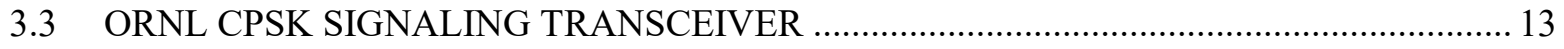

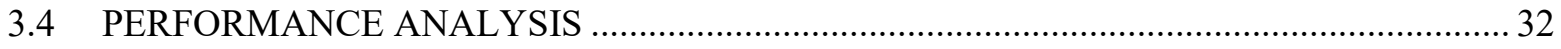

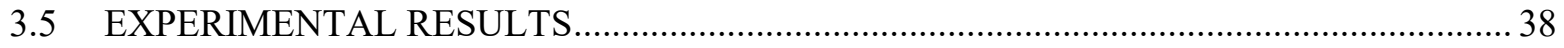

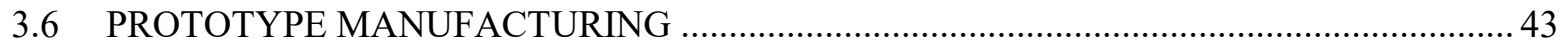

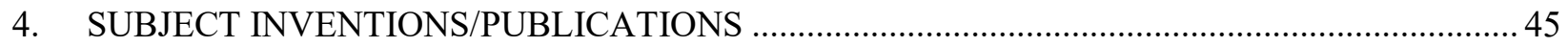

5. COMMERCIALIZATION POSSIBILITIES AND FUTURE COLLABORATION …..................... 46

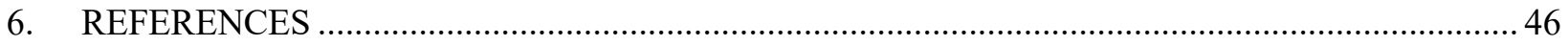

APPENDIX A. LOW-COST WIRELESS SENSORS (MOLEX) TEST DEPLOYMENT ..................... A-1 


\section{INDEX OF ABBREVIATIONS}

$\mu \mathrm{A}$ microAmp

$\mu \mathrm{m}$ micrometer

$\mu \mathrm{Ohm}$ microOhm

AC Alternating Current

AES Advanced Encryption Standard

AGC Automatic Gain Control

AM Amplitude Modulation

AP All Polyimide

ARM Advanced RISC Machines

ASIC Application Specific Integrated Circuit

AWGN Additive White Gaussian Noise

BER Bit Error Rate

bps bit per second

BPSK Binary Phase Shift Keying

C Celsius

CAD Computer Aided Design

CDMA Code Division Multiple Access

$\mathrm{cm}$ centimeter

CPSK Code Phase Shift Keying

CSMA Carrier-Sense Multiple Access

$\mathrm{dB}$ decibel

$\mathrm{dBm}$ decibel-milliWatts

DBPSK Differential Binary Phase Shift Keying

DC Direct Current

F Fahrenheit

FIR Finite Impulse Response

FM Frequency Modulation

FPGA Field Programmable Gate Array

FRP Flexible Research Platform

FSK Frequency Shift Keying

GF Gauge Factor

GPIO General Purpose Input Output

GPS Global Positioning System
HVAC Heating, Ventilation and Air-

Conditioning

$\mathrm{Hz}$ Hertz

IAQ Indoor Air Quality

IFA Inverted $F$ Antenna

IIR Infinite Impulse Response

in inch

K Kelvin

kWh kiloWatt hr

LFSR Linear Feedback Shift Register

$\mathrm{mA}$ milliAmp

MEMS Micro Electro-Mechanical Systems

$\mathrm{MHz}$ MegaHertz

MLS Maximal Length Sequences

$\mathrm{mm}$ millimeter

$\mathrm{mW}$ milliWatt

nm nanometer

OOK On Off Keying

ORNL Oak Ridge National Laboratory

pF picoFarad

PI PolyImide

PN Pseudo Noise

PTP Pulse Thermal Processing

QPSK Quadrature Phase Shift Keying

RF Radio Frequency

RH Relative Humidity

s second

SMA Sub Miniature version A

SNR Signal to Noise Ratio

TDMA Time Division Multiple Access

V Volts

VHDL Very high speed integrated circuit

Hardware Description Language

XOR Exclusive OR

xTea extended Tiny encryption algorithm 


\section{INDEX OF FIGURES}

Figure 1 A multifunctional sensor platform for smart buildings. .......................................................... 1

Figure 2 Sensor platform powered by materials and manufacturing innovations.................................... 2

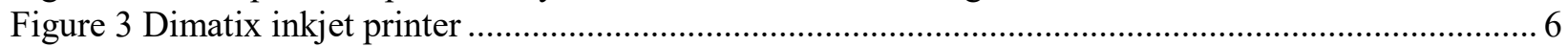

Figure 4 Sheet resistance of silver metal line printed on polyimide substrate ......................................... 6

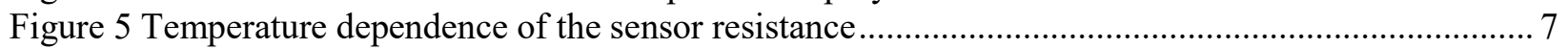

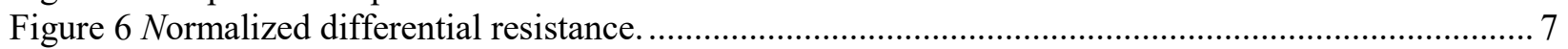

Figure 7 The capacitance of a printed RH sensor has a function of humidity content ............................. 8

Figure 8 Inkjet-printed strain gauge on flexible PI substrate ............................................................. 8

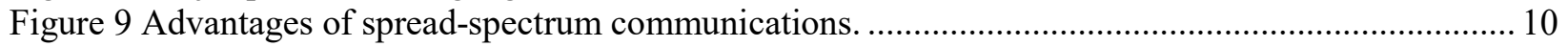

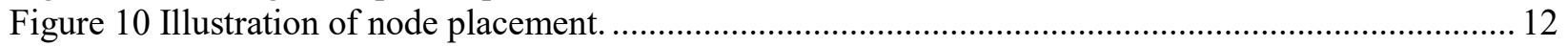

Figure 11 Schematic diagram of the implemented Gold CPSK transmitter............................................ 15

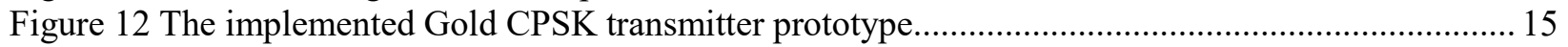

Figure 13 The implemented Kasami CPSK transmitter prototype. ..................................................... 15

Figure 14 The implemented CPSK prototype transmitter: fully functional prototype on FR4 ................. 16

Figure 15 The implemented CPSK prototype transmitter: fully functional prototype on thin film............ 16

Figure 16 Schematic diagram of the implemented Gold CPSK receiver................................................ 17

Figure 17 Schematic diagram of the implemented Kasami CPSK receiver. ............................................. 18

Figure 18 The implemented CPSK prototype of the wireless receiver.................................................. 19

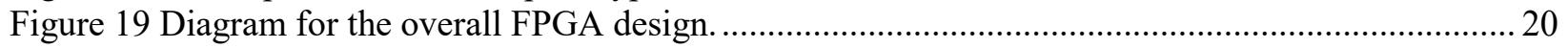

Figure 20 Zoomed diagram for the individual bits of the control functions........................................... 21

Figure 21 Zoomed diagram for the standard Vivado system that connects the Zynq Linux processor

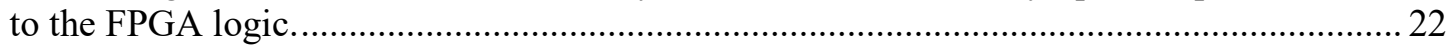

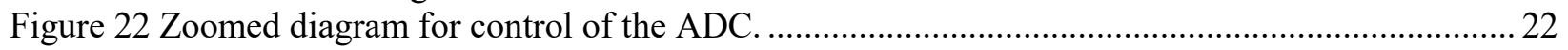

Figure 23 Zoomed diagram for the SimulationInsert block............................................................. 23

Figure 24 Zoomed diagram for generating the Gold code sequence and preamble detection................... 23

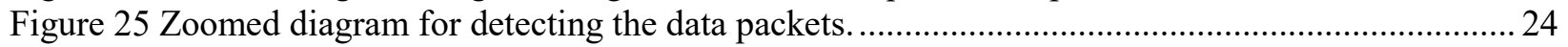

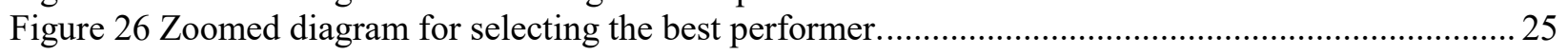

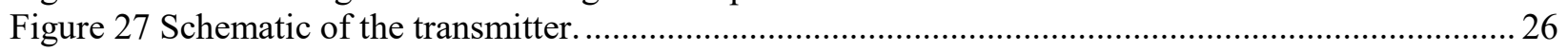

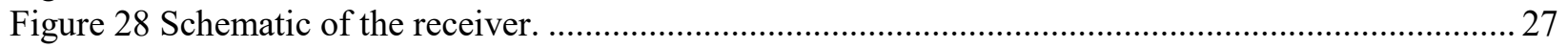

Figure 29 The recommended star-tree topology for sensor deployment. .......................................... 27

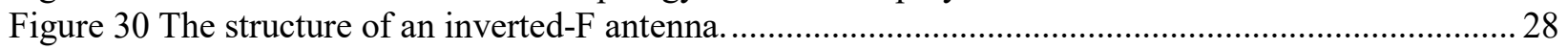

Figure 31 Frequency-dependent dielectric properties of polyimide substrate. .......................................29

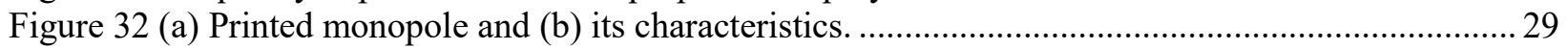

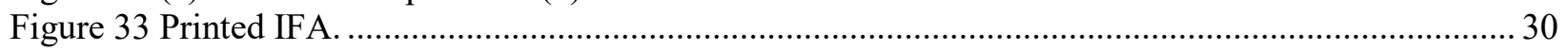

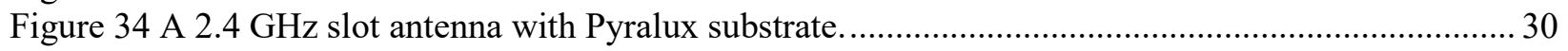

Figure 35 Simulation and measurement results for a $2.4 \mathrm{GHz}$ antenna prototype.................................. 31

Figure 36 A 433 MHz printed planar IFA and monopole............................................................ 31

Figure 37 Single-user BER performance for the CPSK signaling scheme under an AWGN

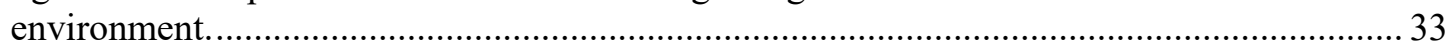

Figure 38 Single-user BER performance for the CPSK signaling scheme under Rayleigh fading-

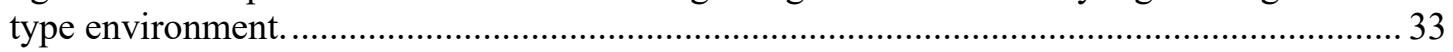

Figure 39 Success rates of the original multiple access scheme at a packet transmission time of 1

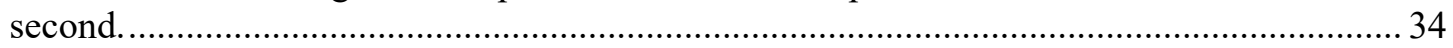

Figure 40 Success rates of the original multiple access scheme at a packet transmission time of 0.1

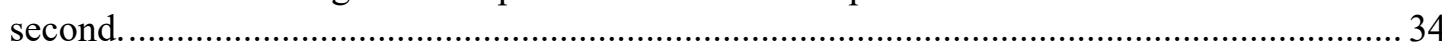

Figure 41 Success rates of the modified multiple access scheme at a packet transmission time of 1 second...... 
Figure 42 Success rates of the modified multiple access scheme under packet transmission time of

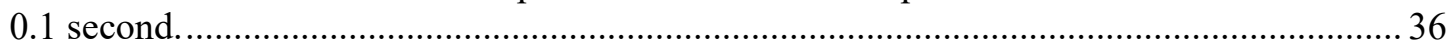

Figure 43 BER performance of the developed CPSK signaling scheme ................................................. 37

Figure 44 BER performance of the developed CPSK signaling scheme due to interference resulting from transmitters at different hubs under a Rayleigh fading-type environment. ..................... 37

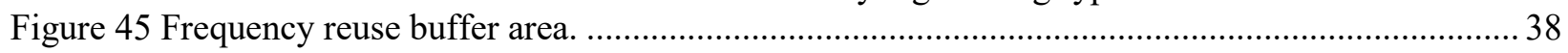

Figure 46 Nine simultaneous users for this scenario would require nine separate frequencies.................. 38

Figure 47 An example showing that frequency reuse in spread-spectrum systems would recover most of the extra bandwidth compared with conventional AM. .......................................... 38

Figure 48 Illustration of the key limitation in the randomness of the phase relationship between the

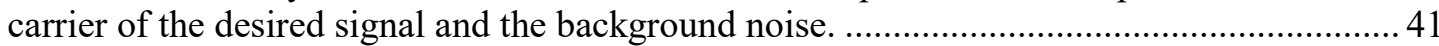

Figure 49 Illustration of the angle $\mathrm{S}$ versus the signal-to-noise ratio $\mathrm{Vs} / \mathrm{Vn}$ in decibels.......................... 42

Figure 50 Coverage range testing on the first floor: packet success rates are measured at different

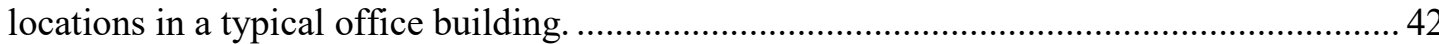

Figure 51 Coverage range testing on the second floor: packet success rates are measured at different

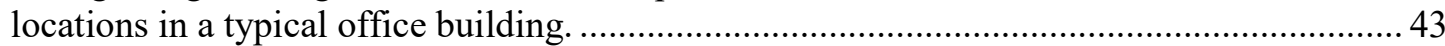

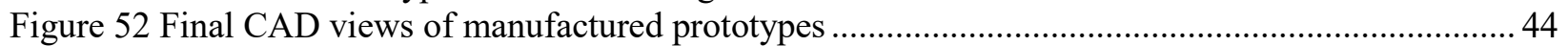

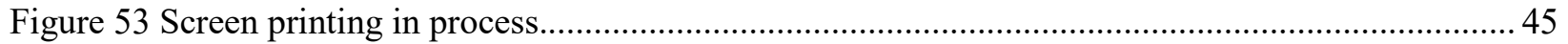

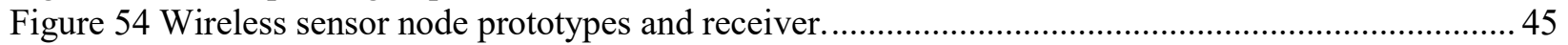

Figure 55. Initial prototype of energy harvesting wireless sensor. .................................................... A-2

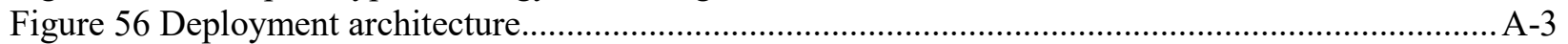

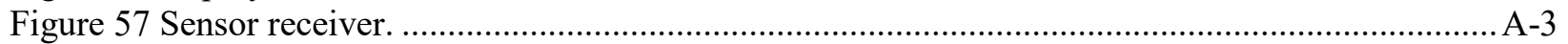

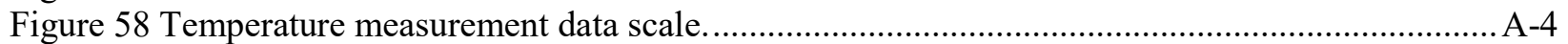

Figure 59 FRP\#1 space temperature measurement sensor locations. .............................................. A-5

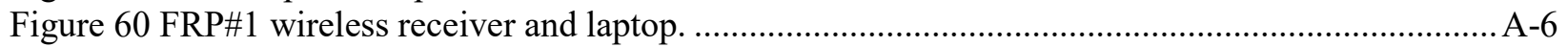

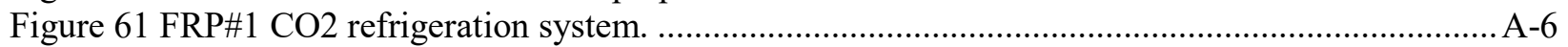

Figure 62 FRP\#1 sensor locations in low-temperature refrigeration case. ...................................... A-7

Figure 63 FRP\#1 sensor locations m medium-temperature refrigeration case..................................... A-7

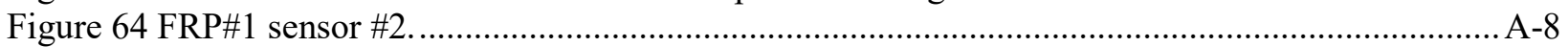

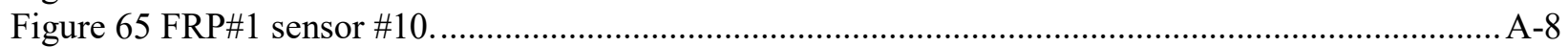

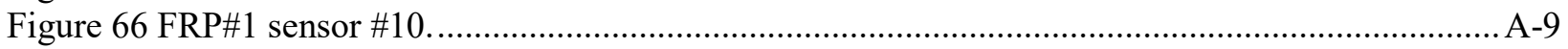

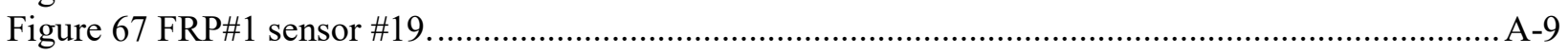

Figure 68 FRP\#1 sensor tags \#1 - \#10 temperature measurement June 30 to July 19 ........................... A-11

Figure 69 FRP\#1 sensor tag \#5 temperature measurement compared with research thermocouple........ A-11

Figure 70 FRP\#1 sensor tag \#6 temperature measurement compared with research thermocouple........ A-12

Figure 71 FRP\#1 sensor tags \#1 - \#10 humidity measurement June 30 to July $19 . \ldots \ldots \ldots \ldots \ldots \ldots \ldots . . . . . . . . . . . . . . A-12$

Figure 72 FRP\#1 sensor tag \#2 humidity compared with research instrumentation June 30 to July

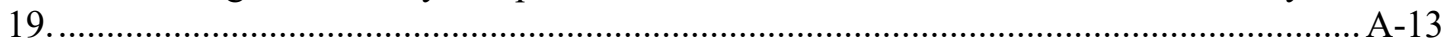

Figure 73 FRP\#1 sensor tags \#1 - \#10 luminosity (lights on/off) June 30 to July 19......................... A-13

Figure 74 FRP\#1 sensor tags \#1 - \#10 luminosity (lights on/off) compared with FRP\#1 lighting

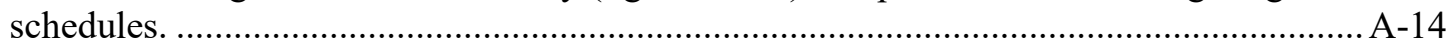

Figure 75 FRP\#1 sensor tags \#11 - \#14 temperature measurements June 30 to July 19........................14

Figure 76 FRP\#1 sensor tags \#11 - \#14 temperature measurements June 30 to July 19......................A-15

Figure 77 FRP\#1 sensor tags \#15 - \#16, \#18 - \#19 temperature measurements June 30 to July 19...... A-15

Figure 78 FRP\#1 sensor tags \#15 - \#16, \#18 - \#19 temperature measurements June 30 to July 19...... A-16

Figure 79 FRP\#1 sensor tags \#11 - \#14, 15 - \#16, \#18 - \#19 humidity measurements June 30 to July 19 in refrigerated case................................................................................... A-16

Figure 80 FRP\#1 sensor tags \#11 - \#14, 15 - \#16, \#18 - \#19 luminosity measurements June 30 to

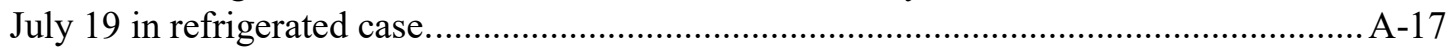

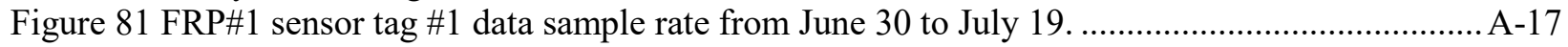

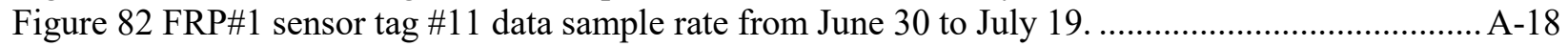


Figure 83 FRP\#1 sensor tag \#1 comparison of data sample rate versus luminosity measurement......... A-18

Figure 84 FRP\#1 sensor tag data transmission event analysis.......................................................... A-19

Figure 85 FRP\#2 space temperature measurement sensor locations on first floor................................. A-20

Figure 86 FRP\#2 space temperature measurement sensor locations on second floor. ............................ A-20

Figure 87 FRP\#2 sensor tag \#1 location (first floor Room 102) .......................................................

Figure 88 FRP\#2 sensor tag \#2 location (first floor Room 106)........................................................ A-21

Figure 89 FRP\#2 sensor tag \#3 location (first floor Room 105) ......................................................

Figure 90 FRP\#2 sensor tag \#5 location (first floor Room 104) ....................................................

Figure 91 FRP\#2 sensor tag \#6 location (first floor Room 104).....................................................23

Figure 92 FRP\#2 sensor tags \#1-\#8 (first floor) temperature measurements July 19-27.................... A-25

Figure 93 FRP\#2 sensor tags \#1, \#3, \#5, \#6 (first floor) temperature measurements July 19-27......... A-25

Figure 94 FRP\#2 sensor tags \#4, \#7, \#8 (first floor) temperature measurements July 19-27................ A-26

Figure 95 FRP\#2 sensor tags \#11-\#17, \#19, and \#20 (second floor) temperature measurements July

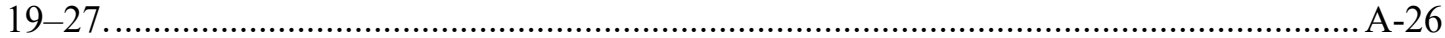

Figure 96 FRP\#2 sensor tags \#9 and \#10 (second floor) temperature measurements July 19-27.........A-27

Figure 97 FRP\#2 sensor tags \#1 - \#8 (first floor) humidity measurements July 19-27........................ A-27

Figure 98 FRP\#2 sensor tags \#9 - \#17, \#19, and \#20 (second floor) humidity measurements July

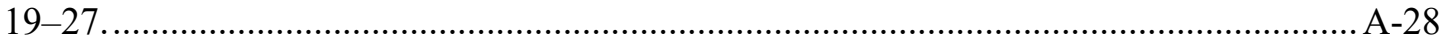

Figure 99 FRP\#2 sensor tags \#1 - \#8 (first floor) luminosity measurements July 19-27.................... A-28

Figure 100 FRP\#2 sensor tags \#9 - \#17, \#19, and \#20 (second floor) luminosity measurements July

$19-27$.

A-29

Figure 101 FRP\#2 sensor tag \#11 (second floor) luminosity measurements July 19-27....................A-29

Figure 102 FRP\#2 sensor tag \#1 data sample rate from June 30 to July 19.........................................

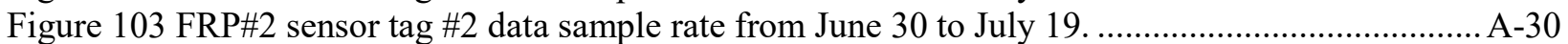

Figure 104 FRP\#2 sensor tag \#11 data sample rate from June 30 to July 19.......................................

Figure 105 FRP\#2 sensor tag \#11 data sample rate from June 30 to July 19.......................................32

Figure 106 FRP\#2 sensor tag data transmission event analysis........................................................ A-33

\section{INDEX OF TABLES}

Table 1 Comparison between state-of-the-art low-power wireless sensor technologies with the

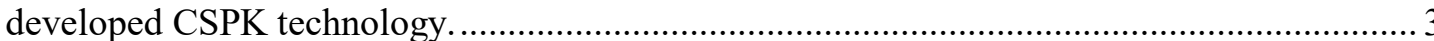

Table 2 The number of available orthogonal codes and their effective process gains for the most

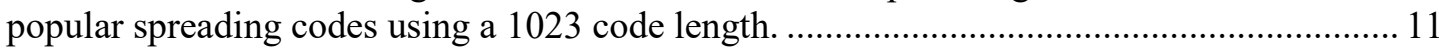

Table 3 Specifications of the implemented CPSK transmitter prototype. ............................................. 16

Table 4 Experimental receiver sensitivity testing for the CPSK prototype using AM signal with a 1023 length spread-spectrum code.

Table 5 Experimental receiver sensitivity testing for the CPSK prototype using FSK modulation signal.

Table 6 The theoretical receiver sensitivity of the CPSK signaling scheme. 



\begin{abstract}
Buildings consume $73 \%$ of the energy produced in the United States [1, 2]. Advanced sensors, controls, and communications have the potential to reduce the energy consumption of buildings by $20-40 \%[3,4]$. Currently, installation and wiring costs for sensors are quite high, making it cost-prohibitive for building managers to deploy large quantities of advanced sensors [5]. Wireless sensors have recently been deployed in buildings to provide the information necessary for optimal control of heating, ventilation, and air-conditioning and lighting systems $[6,7]$. Wireless sensors have the unique advantage of being suitable for easily retrofitting existing buildings at a minimal labor cost, and the flexibility to be placed at optimal, observable locations. However, current commercially available wireless sensors are still very expensive (\$150-300/node). Thus, revolutionary technological improvements in wireless sensors are required to promote inherently low-cost manufacturing for building applications to successfully exploit the energy efficiency opportunities in buildings.

In this report, we present the efforts carried out at Oak Ridge National Laboratory and Molex Inc. toward exploring the design, implementation, and performance evaluation of low-cost, self-powered wireless sensors - including temperature, relative humidity, occupancy, and indoor air quality sensors - to enable advanced control applications that reduce energy consumption in buildings. Our focus is to demonstrate a novel multifunctional sensor platform that will evolve with advances in materials technology, low-cost printing techniques, nano-antennas, and co-integration of monitoring, control, and communication circuitry. We also present a low-power and bandwidth-efficient wireless communication technology that can be driven by energy harvesting in self-powered wireless sensors. It is based on code-phase-shift keying (CPSK) spread-spectrum signaling that improves both transmitter range and power consumption. The objective is to minimize the number of components within the wireless transceiver and reduce power consumption to allow sensor networks powered by energy harvesting, thus eliminating the expense and effort of periodically replacing the sensor batteries. Current state-of-the-art radio technologies have demonstrated 25-30 mA current consumption for the transmitter. Our prototype demonstrations lowered the consumption to $4 \mathrm{~mA}$ using spread-spectrum-based CPSK so that solar cells can be used with indoor lighting to recharge a battery indefinitely. Therefore, the system can run with no attention or maintenance for the life of the components, which is expected to be 20 years. The developed technology has the potential to generate fully printable wireless sensors with costs on the order of $\$ 1-10 /$ node.
\end{abstract}




\section{INTRODUCTION}

Printed electronics are an increasingly mature technology [8]. The emerging industry of large-area manufacturing and organic and printed electronics is bringing new opportunities for the realization of sensors on unconventional substrates. Besides a strong potential for cost-effective production based on additive processes with reduced manufacturing infrastructure, the benefits of printing devices on plastic films include their potential to be lightweight, foldable/rollable, thin, conformal, and manufacturable by roll-to-roll processes. A wide range of sensors and potential building applications can make use of integration on plastic substrates. These advancements in sensor technology have the potential to benefit diverse technology platforms, including building retrofits.

The aim of this research and development of a sensor platform is to establish a high degree of coordination among complexity, functionality, innovation, and expected benefits. It is foreseeable that smart sensors integrated on flexible substrates will evolve toward an all-printable technological solution, taking us toward "peel-and-stick" wireless sensors (see Figure 1). The developed integrated multifunctional sensor platform addresses a multiplicity of energy functions for buildings technology while paving the way for integration of non-energy functions such as safety and security.

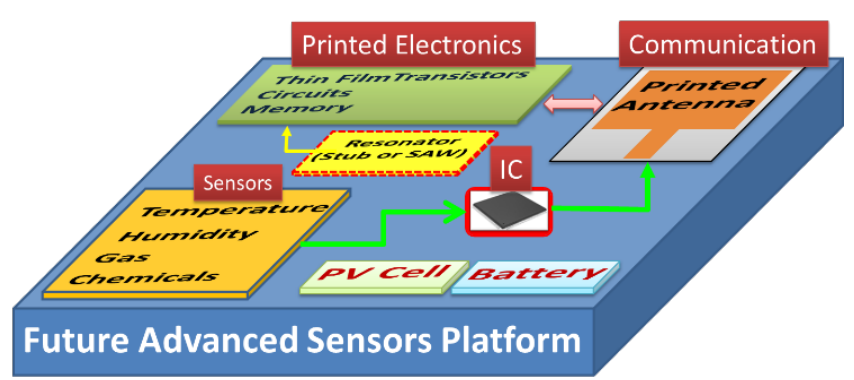

Figure 1 A multifunctional sensor platform for smart buildings.

Developing an integrated system that is fully printable has the following key functional challenges:

- Develop components of a wireless sensor that can be printed together with limited post-processing

- Innovate sensors at low cost that are specific to building monitoring requirements

- Develop low-power platforms that can be driven by energy harvesting

The main challenge for sensors integrated on plastic flexible substrates is to establish a complete technology chain: material development, processing and characterization equipment, and production in an energy-efficient and economically feasible manner compared with existing manufacturing techniques. Our focus is to demonstrate a novel multifunctional sensor platform that will evolve with advances in materials technology, low-cost printing techniques, and co-integration of monitoring, control, and communication circuitry. These are summarized in Figure 2 as core strengths: materials innovations, rollto-roll manufacturing capabilities, and pulse thermal processing. Successful integration of a multifunctional smart sensor system will address the key limitations of current materials and device technologies to realize a low-cost, multifunctional sensor platform. 


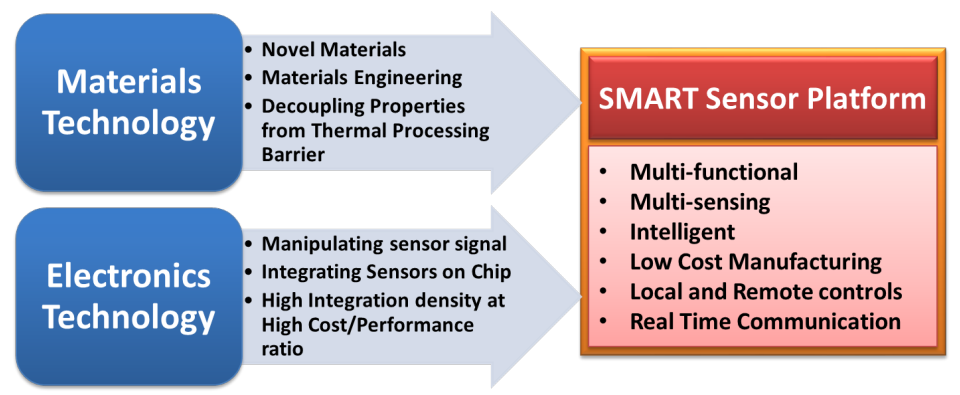

Figure 2 Sensor platform powered by materials and manufacturing innovations.

Oak Ridge National Laboratory (ORNL) has developed a prototypic sensor platform using printable technologies to enable low-cost roll-to-roll manufacturing of sensor devices. A platform with temperature and humidity sensors has been demonstrated. This technology is aimed at demonstrating the feasibility of low-cost, low-power wireless sensors - including temperature, relative humidity, occupancy and indoor air quality (IAQ) sensors - to enable advanced control applications to reduce energy consumption in buildings. These control strategies include (1) occupancy-based control of building systems to condition building spaces only when occupants are present and (2) demand-controlled ventilation to improve the air quality within buildings for a healthier environment. This research has the potential to improve energy efficiency in buildings and position US manufacturers to play a dominant role in the flexible printable sensor industry, projected to be a $\$ 44.3$ billion market in 2021 compared with $\$ 2.2$ billion in 2012 . The outcome of this research is the demonstration of a low-cost multi-modal wireless sensor network for deployment in buildings to enable next-generation controls focused on dramatically increasing the energy efficiency of existing buildings.

According to the Commercial Building Energy Consumption Survey, less than 10\% of the buildings in the United States use building automation systems or central controls to manage their building system operations [9]. Typically, large buildings $\left(>100,000 \mathrm{ft}^{2}\right)$ use building automation systems, while over $90 \%$ of existing buildings are either small or medium sized and have no cost-effective way to monitor and control building systems. Typically, dedicated thermostats that control packaged rooftop units control these buildings. Buildings with automation systems possess building automation networks that integrate sensors, actuators, and controllers within the building. However, integrating new sensors into these automation networks is challenging because of interoperability issues and oftentimes sub-optimal wiring locations (resulting in sub-optimal sensor locations for collecting observations). Buildings without automation networks would face significant expense for wiring their systems to support increased sensing. Currently, installation and wiring costs for sensors are high, making it cost-prohibitive for building managers to deploy large quantities of advanced sensors. Wireless sensors that can be used for buildings are still expensive (\$150-\$300/node).

With energy prices forecast to rise for the foreseeable future and increasing interest in environmentally responsible "green" buildings, it is critical that such buildings be energy efficient. New technologies involving self-powered wireless communication technologies along with smart sensors are key to addressing the energy and resource waste issues of current buildings. The ability to directly print many sensors in parallel leads to sensors that are very inexpensive, opening new markets and creating jobs in sensor manufacturing and many downstream industries that would benefit from deployment.

Development of this technology will enhance the energy efficiency, productivity, and agility of manufacturing processes, as well as buildings.

One important consideration is the economics of deploying smart building technologies. Smart building systems (such as sensors and actuators) cost money to deploy and maintain. Thus, the return on investment must be adequate for any organization to pay for the sensor systems. Based on a building case study, the total cost for installation can be approximately the same as the yearly energy savings, at 
$\$ 0.13 / \mathrm{kWh}$. This means a building can recover the installation costs in one year through the reduction in energy usage. Although the return will be different for every building, smart building systems are extremely economical and provide monetary value for buildings that choose to invest in them. In the United States, lighting consumes $22 \%$ of electricity and represents $\$ 40$ billion a year in energy costs. Using advanced technologies like occupancy sensors has the potential to reduce unnecessary lighting use by 13 to $90 \%$ for a significant return on investment.

Self-powered wireless sensors that can be fully integrated into a building energy management system provide a unique opportunity to rapidly deploy whole-building monitoring solutions in a retrofit fashion. Although sensors do not directly save energy, they provide the basis for enhanced control of buildings based on real-time energy usage, rather than on design-day decisions made when the building is built. Correlating the control actions taken by buildings based on indoor and outdoor air temperature, level of occupancy, holiday schedules, IAQ, and energy prices has the potential for significant reductions in building energy usage. The developed technology has the potential to realize such sensor systems at low cost to improve building energy efficiency and provide a return on investment in less than 2 years.

A key area in which wireless sensor performance improvement is advantageous is the power consumption and transmit range. Current state-of-the-art low-power wireless sensor technologies [10-15] consume 10$100 \mathrm{~mW}$; therefore battery replacement is required from time to time. Thus, current technologies require regular maintenance and so are not cost-efficient. The communication technology developed in this effort is based on code-phase-shift keying (CPSK) spread-spectrum signaling [16], which improves both power consumption and transmitter range. The objective is to minimize the number of components within the wireless transceiver and reduce power consumption to allow sensor networks to be powered by energy harvesting, thus eliminating the expense and effort of periodically replacing the sensor batteries. The battery consumption of the developed technology is very low $(1-5 \mathrm{~mW})$ so that solar cells can be used with indoor lighting to recharge a battery indefinitely. Thus, the system can run with no attention or maintenance for the life of the components, which is expected to be 20 years. The developed technology has the potential to generate fully printable wireless sensors with costs on the order of $\$ 1-10$ /node.

In this report, we present the effort carried out by ORNL and manufacturer Molex Inc. in exploring the design, implementation, and performance evaluation of low-cost, self-powered wireless sensorsincluding temperature, relative humidity, occupancy and IAQ sensors - to enable advanced control applications that reduce energy consumption in buildings. Our focus was to demonstrate a novel multifunctional sensor platform that can evolve with advances in materials technology, low-cost printing techniques, nano-antennas, and co-integration of monitoring, control, and communication circuitry. We also present a low-power, bandwidth-efficient wireless communication technology that can be driven by energy harvesting in self-powered wireless sensors. It is based on CPSK spread-spectrum signaling that improves both transmitter range and power consumption. Experimental testing was conducted, in addition to end-to-end system-level simulation-based studies, conducted to evaluate the performance of the proposed Gold/Kasami CPSK scheme. The realized performance metrics include receiver sensitivity, coverage range in free space and typical office building environments, average probability of bit-error over standard Gaussian and Rayleigh fading-type channels, and multiple access capabilities. The specific engineering tradeoffs are also discussed. Table 1 illustrates the major advantages of the implemented Gold/Kasami CPSK transceiver.

Table 1 Comparison between state-of-the-art low-power wireless sensor technologies with the developed CSPK technology.

\begin{tabular}{|l|l|l|}
\hline \multicolumn{1}{|c|}{ Feature } & \multicolumn{1}{c|}{ State-of-the-art } & \multicolumn{1}{c|}{ CPSK } \\
\hline Current consumption & $\sim 20-30 \mathrm{~mA}$ per Tx & $\sim 3-5 \mathrm{~mA}$ per Tx \\
\hline Frequency & $2.4 \mathrm{GHz}$ & $433 \mathrm{MHz}$ \\
\hline Output power & $10-100 \mathrm{~mW}$ & $1-5 \mathrm{~mW}$ \\
\hline
\end{tabular}




\begin{tabular}{|l|l|l|}
\hline Receiver sensitivity & $-95 \mathrm{dBm}$ & $-144 \mathrm{dBm}$ \\
\hline Modulation & CSMA, OQPSK & CDMA, CPSK \\
\hline Processing gain & $\sim 9 \mathrm{~dB}(16)$ & $\sim 30 \mathrm{~dB}(1023)$ \\
\hline Range (free space) & $\sim 0.5$ mile & $\sim 0.68$ mile \\
\hline
\end{tabular}

\section{STATEMENT OF OBJECTIVES}

The objective of this project is to work with a manufacturer to demonstrate a path toward low-cost manufacturing of the wireless sensor networking technology developed at ORNL and improve the performance of the laboratory prototype to meet deployment requirements within buildings. The targeted improvements for the final prototype include 1) ultra-low power consumption of the wireless communication, 2) multi-parameter sensing platform, and 3) on-board computational data processing

Wireless sensor designs that leverage advanced low-cost manufacturing techniques along with innovative self-powered techniques have the potential to improve the ease of deployment of sensors and controls within buildings to optimize energy use along with identifying and diagnosing faults. This project provides a path toward driving down costs and potentially inviting "non-traditional suppliers" into the market by increasing the size of the market and improving competition and the rate of innovation. Wireless sensors have the unique advantages of suitability for easy retrofitting of existing buildings at a minimal labor cost, and the flexibility to be placed at optimal observable locations. However, current commercially available wireless sensors are still too expensive for building owners to make a business case for installing them, and their use requires expertise in installation and configuration management. The platform resulting from this project will offer direct and immediate value in the following ways:

1. Building owners/tenants can deploy these systems to monitor the environment in buildings and can modify control systems to manually or automatically include the sensor data for optimal energy usage by heating, ventilation, air conditioning (HVAC) and lighting equipment.

2. Building energy services companies can use these sensors as part of energy savings performance contracts to identify energy efficiency improvement opportunities.

3. HVAC systems, control systems, and lighting manufacturers can use these sensors as retrofit kits to better optimize their packaged rooftop units or lighting control systems based on occupancy and the indoor building environment.

4. Wireless sensor manufacturing companies can produce these sensors for retrofit installation into existing buildings.

The objectives of the project are

1. Evaluate manufacturing requirements and design options for developing self-powered, multi-sensor wireless platforms, including commercially available, temperature, humidity, and light sensors for use in buildings.

2. Produce manufacturing prototypes of the wireless sensors using innovative manufacturing techniques.

3. Evaluate the performance of the prototypes and improve platform design to achieve compact, lowcost, self-powered wireless platform applicable to building requirements. 


\section{TECHNICAL DISCUSSION}

\subsection{MATERIALS DEVELOPMENT AND PROCESSING}

Inkjet printing, currently one of the cheapest direct-write techniques, is moving from the laboratory scale to the manufacturing scale. There is real interest in producing sensors on plastic foil using large-area manufacturing techniques. Besides a strong potential for cost-effective production based on additive processes with a reduced infrastructure, the benefits of printing devices on flexible substrate include their potential to be lightweight, foldable/rollable, transparent, thin and conformal, wearable, and produced at large scale, depending on the processing technology involved.

The additive inkjet printing process involves direct deposition of a precursor on a suitable substrate to form functional components in a user-selected, well-defined area. Multiple techniques are available for depositing functional materials on suitable substrates. The main techniques of interest for integration on flexible substrate include drop casting, screen printing, spin coating, and semiconductor microfabrication. All these techniques allow flexible spatial control of the material deposition process. However, when it comes to achievable resolution, there are significant differences: Drop casting is suitable for rapid material processing and evaluation; however, it is not scalable and does not offer device geometry control. Drop casting is also inefficient in terms of material usage compared with inkjet printing. In screen printing, the target material is deposited onto the substrate by forcing the "ink" through the pores of the "screen," which defines the outline of the printed feature. It is a very fast and a low-cost printing method. The feature size can be controlled down to $20 \mu \mathrm{m}$ with the currently available technology. However, it has several drawbacks compared with inkjet printing: low achievable film thickness control, limited choice of shape and layout, no spatial control using a single mask, and substantial material wastage. In some cases, screen printing can be applied in combination with inkjet printing to profit from the advantages of both technologies. Spin coating is a fast and simple method for depositing thin, uniform layers of a material on comparably large areas. However, it does not offer the possibility of spatial control and therefore cannot compete with inkjet printing in terms of resolution and flexibility. Furthermore, it is not very economical in material use because a significant amount of the coating liquid does not remain on the substrate but is wasted. In contrast, various lithographic techniques are superior to inkjet printing in terms of achievable resolution. However, their subtractive character calls for a larger number of processing steps; and the selective removal of pre-deposited material requires a specific mask for every pattern, resulting in lower flexibility, throughput, and cost-efficiency.

Drop-on-demand inkjet printing technology, with its efficient use of ink, has become an attractive alternative for the fabrication of interconnects and sensors, especially for research and prototyping applications for which pattern flexibility is highly desired. Research and development efforts worldwide are focused on exploiting additive printing techniques to integrate an entire sensing system with a strong potential for cost reduction. If inkjet deposition is combined with low-cost substrates, the cost of a final sensor would be mostly determined by the measurement electronics, rather than the sensing materials. Drastically reducing the prices of electronic devices would enable the development of a next generation of ubiquitous electronic systems.

The inkjet printing technique was explored for the development of low-cost resistive and capacitive sensors for buildings applications. Figure 3 shows a picture of the Dimatix materials printer used in this project. FUJIFILM Dimatix has leveraged its piezoelectric inkjet technology and MEMS (micro-electromechanical systems) fabrication processes to produce a materials printer specifically designed for research and development and feasibility testing. The DMP-2831 printer allows the deposition of fluidic materials on an $8 \times 11$ inch or A4 substrate using a disposable piezo inkjet cartridge. This printer can create and define patterns over an area of about $200 \times 300 \mathrm{~mm}$ and handle substrates up to $25 \mathrm{~mm}$ thick with an adjustable $\mathrm{Z}$ height. The temperature of the vacuum platen, which secures the substrate in place, can be adjusted up to $60^{\circ} \mathrm{C}$. The DMP-2831 system with waveform editor and a drop-watch camera 
allows manipulation of the electronic pulses to the piezo jetting device for optimization of the drop characteristics as the drop is ejected from the nozzle. This system enables easy printing of structures and samples for process verification and prototype creation. The printer uses $1.5 \mathrm{ml}$ ink cartridges. Each single-use cartridge has 16 nozzles linearly spaced at 254 microns with typical drop sizes of 1 and 10 pico-liters. Figure 4 shows the electrical performance of a $1 \mu \mathrm{m}$ thick silver line printed on a flexible polyimide (PI) substrate. A sheet resistance value of $1.6 \mathrm{ohm}^{2}$ was obtained for silver lines after curing at $100^{\circ} \mathrm{C}$. The sheet-resistance value was found to decrease rapidly with an increase in annealing temperature in the range of $100-250^{\circ} \mathrm{C}$, and a value close to the bulk value was obtained after annealing at $250^{\circ} \mathrm{C}$. The bulk resistivity of the printed silver was found to be lower than $100 \mu \mathrm{Ohm} / \mathrm{cm}$ after annealing at $150^{\circ} \mathrm{C}$, which is suitable for printed sensor and circuit development.

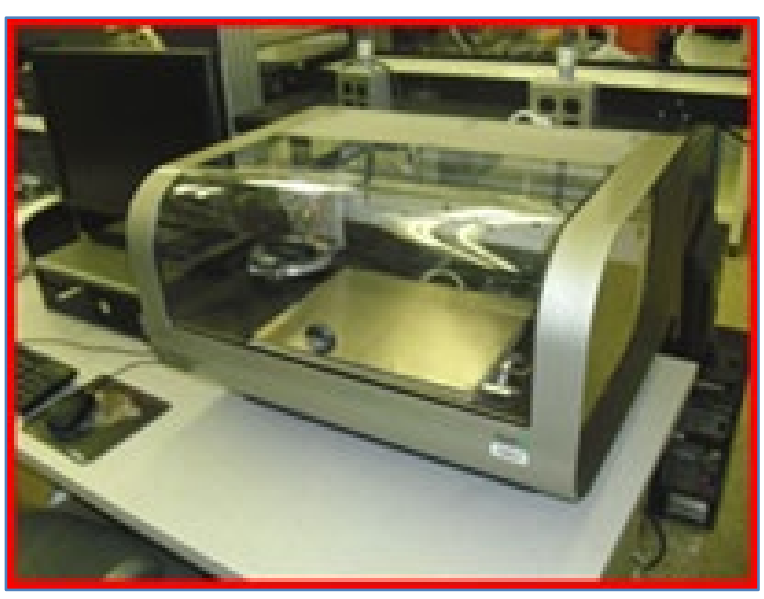

Figure 3 Dimatix inkjet printer

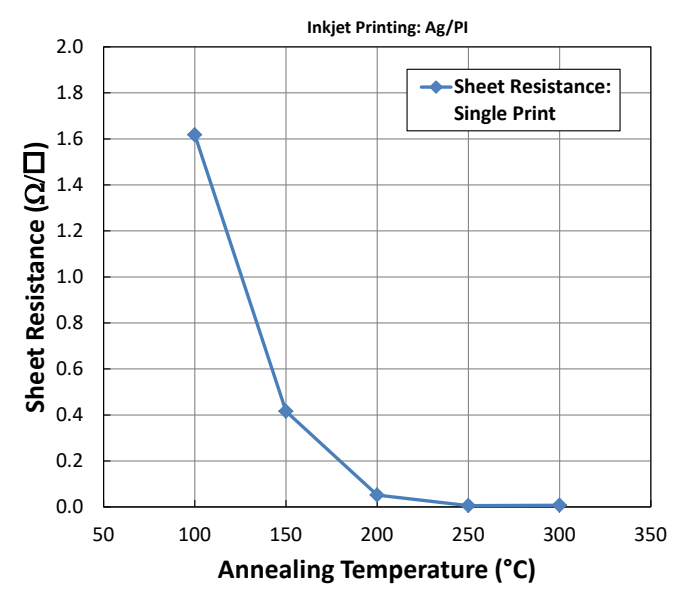

Figure 4 Sheet resistance of silver metal line printed on polyimide substrate

The high-density plasma arc lamp facility at ORNL offers world-leading pulse thermal processing (PTP) capabilities. The plasma arc lamp can produce extremely high power densities of up to $20 \mathrm{~kW} / \mathrm{cm}^{2}$ over areas beyond $1000 \mathrm{~cm}^{2}$. The stable plasma can achieve a temperature of more than $10,000 \mathrm{~K}$ and produces a broad radiant spectrum with wavelengths ranging from 0.2 to $1.4 \mu \mathrm{m}$ (ultraviolent to infrared). The general benefits of PTP with the plasma arc lamp are low thermal budget, increased throughput, higher heating rates, and the ability to process on lower-temperature substrates. ORNL's processing and manufacturing equipment complements its characterization equipment and is versatile enough to work with a variety of material compositions and chemistries. The instruments available are shared among a variety of projects, with prioritization based on milestone deadlines and communication with the instrument scientist. ORNL has developed and patented PTP technology that uses intense radiant energy to heat the surfaces of materials by as much as $600,000^{\circ} \mathrm{C} / \mathrm{s}$ over a large area $\left(\sim 1,000 \mathrm{~cm}^{2}\right)$. The energy can be delivered in pulses of less than $1 \mathrm{~ms}$, providing a steep thermal gradient near the material surface that can be used for high-temperature processing of thin film materials on low-temperature substrates. This technology is commercially available through an industry partner, NovaCentrix, and has demonstrated the ability to dry and sinter printed metallic lines on low-cost polymer substrates in a rollto-roll format. This research has extended the PTP technology to functional electronic devices.

Roll-to-roll technology has long been envisioned as a low-cost manufacturing technology for a number of electronic systems. However, the realization of this potential has been hampered by a lack of material systems that are of sufficient quality and that deliver performance comparable to that of roll-to-roll processing when they are made into devices. Most notably, the lack of high-performance and solutionprocessable semiconductor, insulating, and conductor materials is a key requirement that has still not been adequately met. Current efforts in this area revolve around the development of inorganic and organic 
semiconductors (most notably amorphous oxide materials and small molecule polymers) by solution processing. However, current best results are still constrained by limitations in both material synthesis and device integration. Therefore, the emphasis of this work is on improving the quality of sensor materials and optimizing the integration process toward constructing a device that rivals the performance of devices made by conventional fabrication technology.

Inkjet printing technology is suitable for additive integration of high-performance materials on low-cost, low-temperature flexible substrates. The printing technique allows for rapid material development and evaluation, and prototype and sample generation. The DMP-2831 offers a variety of patterns using a pattern editor program. This system enables easy printing of structures and samples for process verification and prototype creation. Some of the important advantages of inkjet printing that make it an alternative to photolithography and other fabrication methods for fabricating micro- and nano-electronic devices are its compatibility with various substrates, ability to deposit a precise amount of material in a quick and reproducible manner, computer-controlled ability to print on specific locations, lowtemperature processing with no need for vacuum, noncontact and low-cost fabrication method, possibility of finer design and details than screen printing, and ability to print more than one material simultaneously.

Temperature sensor: Resistive temperature sensors were printed on polyimide substrates at a silver drop-spacing of $15 \mu \mathrm{m}$. The line width was maintained at $500 \mu \mathrm{m}$ for the present samples. Figure 5 and Figure 6 show the temperature dependence of a resistive sensor. As illustrated in Figure 5 the resistance of the sensor was found to scale well with the length of the resistive line. The resistance value showed a linear dependence on the measurement temperature in the range of $20-80^{\circ} \mathrm{C}$. The temperature coefficient of the resistance value was found to be the same for both the single-element and series-connected temperature sensors. The temperature coefficient of the resistance was calculated to be $1.5 \times 10^{-3} /{ }^{\circ} \mathrm{C}$, indicating the formation of a dense silver line using nanoparticle ( $<150 \mathrm{~nm}$ in size) ink.

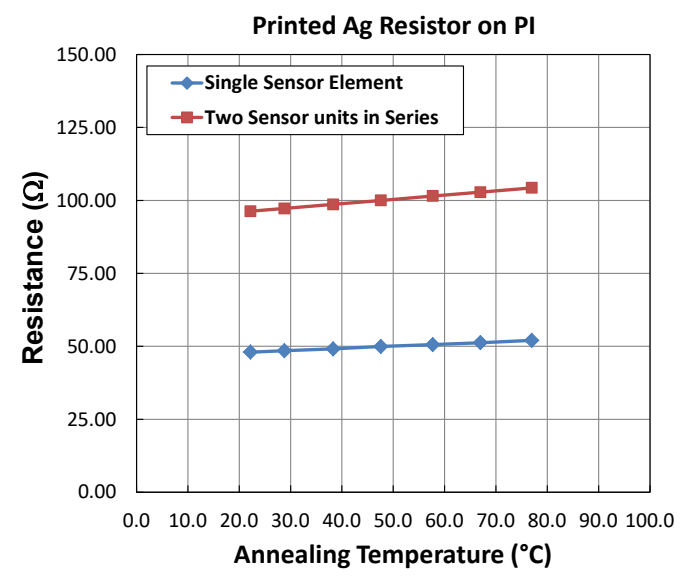

Figure 5 Temperature dependence of the sensor resistance

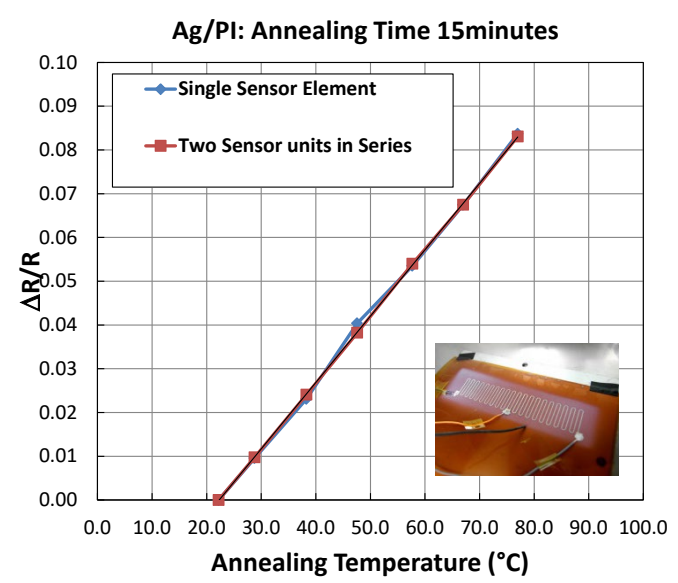

Figure 6 Normalized differential resistance.

Humidity sensor: Capacitive relative humidity (RH) sensors were developed on 7. $5 \mu \mathrm{m}$ thick PI films. PIs have both chemical stability and long-term stability in the presence of moisture and heat, in addition to good hygroscopic and dielectric properties. Operation of capacitive RH sensors is based on the change of the dielectric constant of a sensitive material as a function of the RH in the surrounding environment. The inkjet technique offers the significant advantage of additive integration of an electrode mesh for sensor capacitance design, eliminating the metal patterning and etching steps that would be required in a semiconductor clean-room setup. The sensor capacitance was defined by the top electrode-mesh area. The humidity sensor design incorporated two different configurations using the inkjet printing approach: (1) single capacitive sensor with absolute capacitance value for detection and (2) printing of both a reference 
sensor and a capacitive sensor to use the differential capacitance for humidity detection. Figure 7 shows the sensor capacitance as a function of humidity content. The measurements were made in a customized chamber using a humidifier with no control of the humidity content. The sensor showed a rapid response on the time scale of $120 \mathrm{~s}$. The measurements conducted on different days showed similar responses, indicating stable RH sensing characteristics. The capacitive value does not depend on the resistance of the printed electrode and is expected to be stable as a function of time. The sensitivity of the RH sensor was found to be about $0.69 \mathrm{pF} / \% \mathrm{RH}$. Further measurements were conducted in an environmental chamber to control the rate of rise of humidity content to better define the RH response in the discrete and differential modes.

Strain gauge: Additive printing techniques offer cost-effective sensor and actuator integration. Rapid prototyping manufacturing techniques and novel materials offer the unique possibility of combining functional and structural elements. We tried to evaluate the strain gauge characteristics of a printed strain gauge, as shown in Figure 8, integrated on a polyimide substrate and mounted on a thin sheet of Al 606. The gauge factor (GF) of the printed gauge was about 2.22, which matches well with the typical GF (2-5) of metallic foils. The observed results are promising for the realization of a print-on-demand technology for diverse commercial and industrial applications.

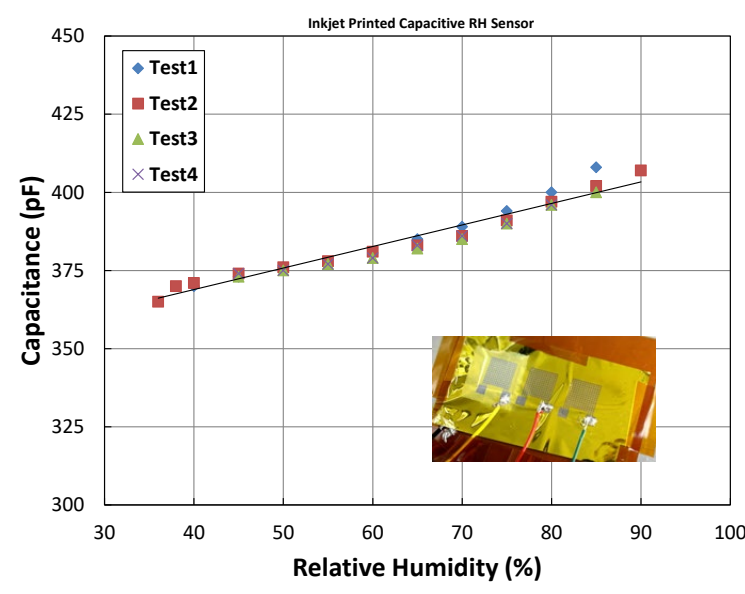

Figure 7 The capacitance of a printed RH sensor has a function of humidity content

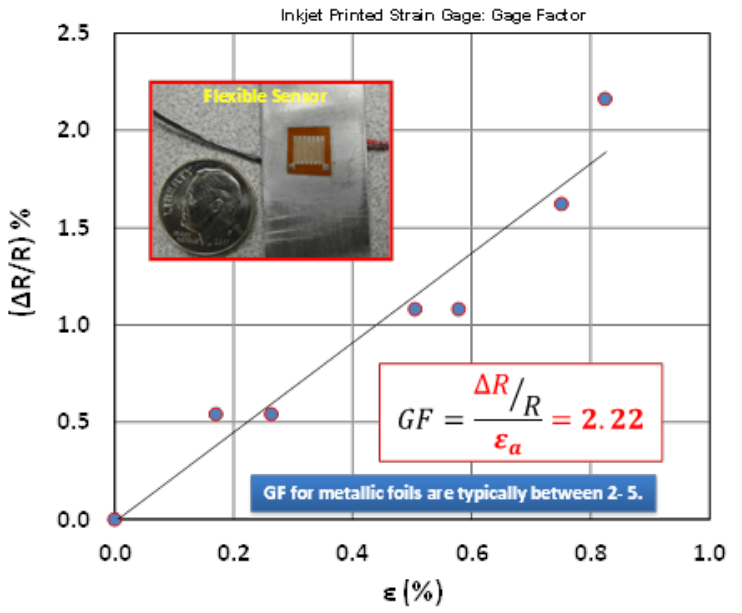

Figure 8 Inkjet-printed strain gauge on flexible PI substrate.

\subsection{COMMUNICATION TECHNOLOGY}

Advanced sensors, communications, and controls can play an important role in reducing the energy consumption of smart buildings. Currently, installation and wiring costs for sensors are quite high, making it cost-prohibitive for building managers to deploy large quantities of advanced sensors. Wireless sensors have recently been used in buildings to provide the information necessary for optimal control of HVAC and lighting systems. Wireless sensors have the unique advantages of suitability for easy retrofitting of existing buildings at a minimal labor cost and the flexibility to be placed at optimal observable locations. Thus, revolutionary technological improvements in wireless sensors are required to promote inherently low-cost manufacturing for building applications to successfully exploit the energy efficiency opportunities in buildings. In this report, we illustrate a low-power bandwidth-efficient communication scheme that can be driven by energy harvesting in self-powered wireless sensors.

The proposed communication technology is based on Gold/Kasami CPSK spread-spectrum signaling that improves both transmitter range and power consumption at a reasonable cost. The objective is to minimize the number of components within the wireless transceiver and reduce power consumption to 
allow sensor networks powered by energy harvesting, eliminating the expense and effort of periodically replacing the sensor batteries. Current state-of-the-art radio technologies have demonstrated 25-100 mA current consumption of the transmitter. Our prototype demonstrations lowered the consumption to $4 \mathrm{~mA}$ using spread-spectrum-based CPSK.

In this section of the report, we present the efforts to explore the design, implementation, and performance evaluation of a hardware prototypic Gold/Kasami CPSK spread-spectrum radio transceiver. Experimental testing in addition to end-to-end system-level simulation-based studies are conducted to evaluate the performance of the Gold/Kasami CPSK in terms of the receiver sensitivity, coverage range in free space and typical office building environments, average probability of bit-error over standard Gaussian and Rayleigh fading-type channels, and multiple access capabilities. The specific engineering tradeoffs are also discussed.

Spread-spectrum CPSK signaling: In this section, we briefly present spread-spectrum CPSK concepts relevant to our work. We show how spread-spectrum modulation can improve both power consumption and transmitter range. For more details on this topic, we refer the reader to references [16, 18-20]. Power consumption and transmitting range are two key areas in which wireless sensor performance improvement is valuable. Current state-of-the-art low-power wireless sensor technologies [6, 7, 10-15] consume 10-100 $\mathrm{mW}$, so batteries must occasionally be replaced. These current technologies, with their requirements for regular maintenance, are not cost-efficient.

The spread-spectrum CPSK signaling technique was selected because it has low peak power requirements - $4 \mathrm{~mA}$ for data transmission in prototype demonstrations [21]. A tenfold improvement in power level and battery consumption was achieved, compared with quadrature phase shift keying (QPSK). Reducing peak power requirements for the transmitter would significantly reduce the transmitter cost as well as reduce interference with other radios. CPSK also has a lower radio frequency (RF) bandwidth requirement than standard spread-spectrum techniques. Other benefits inherent in from spread-spectrum systems include resistance to interference, difficulty of eavesdropping, and reduced requirements for oscillator frequency stability. Figure 9 shows the advantages of spread-spectrum communications with regard to sources of interference. Inherent to spread-spectrum methodology is that several radios can use the same frequency at the same time if different radios use different spread-spectrum codes. This technology, called code division multiple access (CDMA), is central to cell phone and global positioning system (GPS) applications. Although spread-spectrum modulation initially increases the bandwidth required for the transmitter, it has been shown that CDMA techniques can be used to recover much of the increased bandwidth so that the overall system does not consume more spectrum than a comparable QPSK system. 


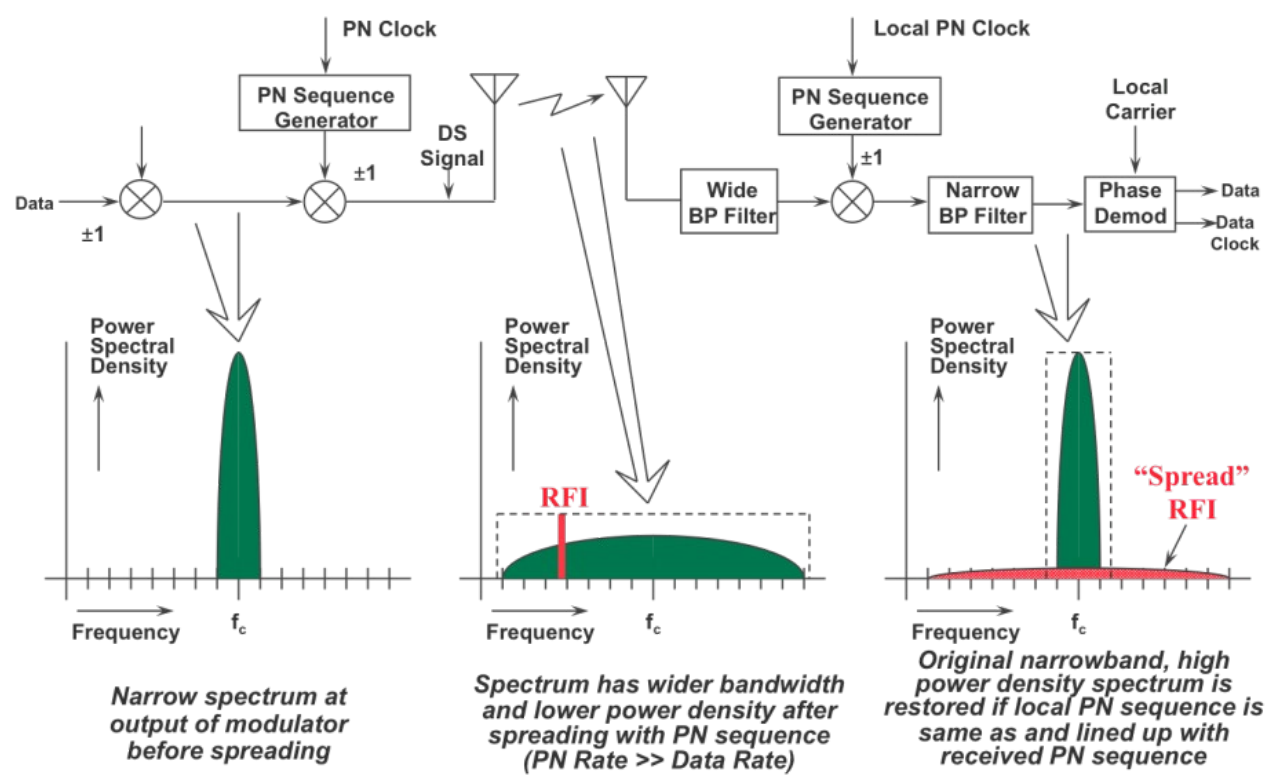

Figure 9 Advantages of spread-spectrum communications.

Basic spread-spectrum systems use a pseudo-noise (PN) random bit stream XORed with the data stream to widen the bandwidth of the signal. At the receiving end, the same pseudorandom signal is XORed (exclusive ORed) with the received signal to reproduce the original data stream. The PN random bit stream rate is usually several times higher than the data rate. A ratio of 1023:1 is typical for spreadspectrum signals and is the rate used in this paper. The XOR process at the receiver acts as a correlator, which performs an accumulating integral on the signal, resulting in a process gain that is equal to the ratio of the spread-spectrum rate to data rate. For example, the 1023:1 spread-spectrum rate produces a process gain of 1023:1, or roughly 30dB. However, since the receiver must be 1023 times wider to accept the modulated signal, the received background noise is also 1023 times greater, so the overall signal-to-noise ratio (SNR) is the same as a regular (single-level logic) QPSK signal. The process gain does help in rejecting interference, which will be rejected by the 1023:1 ratio. This process gain will also allow simultaneous transmissions using CDMA by providing better rejection to interference from neighboring nodes.

The CPSK signaling technique is specially designed to increase the transmission efficiency of spreadspectrum systems and to overcome the spreading gain versus data rate limitation [21]. Circularly shifting the start time (phase) of the spread-spectrum PN bit stream (code) within the packet allows additional modulation. This technique implies circularly shifting each transmitted PN code to represent, with each circularly shifted version of the PN code, a different CPSK symbol mapping a fixed quantity of bits. Therefore, if each period of the data channel PN-code is equal to the duration of the data symbol, the bit transmission rate is increased proportionally to the number of bits mapped by a CPSK symbol [19]. Basic CPSK allows the spread-spectrum stream to be shifted in increments of 1 bit, so in the 1023 bit example, there would be 1023 possible positions with which 10 bits of data could be encoded. Since the signal will include both an in-phase and quadrature components, 20 bits of data can be encoded. Because a comparable signal-strength QPSK signal encodes only 2 bits of data, the CPSK system can provide 10 times the throughput versus a QPSK signal of the same strength. This improvement contributes to the energy required per delivered bit—or the energy efficiency — of the transmission.

Another advantage of spread-spectrum systems is that the wide bandwidth signal requires less accuracy for the center frequency of the signal. Continuing with the 1023 CPSK example, the bandwidth of the spread-spectrum signal will be 102.3 times wider than a QPSK system of the same throughput. If the receiver bandwidth must be a certain percentage ( $10 \%$ for example) greater than the signal bandwidth to 
accommodate the transmitter versus receiver frequency mismatch, the spread-spectrum system will require 102.3 times less frequency accuracy than an equivalent QPSK system. Although this advantage is initially about reducing the cost of the radio oscillators, it also allows the spread-spectrum system to operate at extremely low bit rates if the needed throughput rate is low. The key advantage of the lower bit rate is that, as the radio bandwidth is reduced, the transmitter power required to achieve the same SNR is correspondingly reduced. For example, the experiments conducted at ORNL transmit a single 1023 bit Gold code sequence at a chip rate of 2048 chips per second using amplitude modulation (AM) and require $4000 \mathrm{~Hz}$ of bandwidth. This transmission requires 0.5 seconds and delivers approximately 20 bits of data for an effective data rate of 40 bits per second. An equivalent-rate QPSK system would require $40 \mathrm{~Hz}$ of bandwidth. Good design practice would require that the QPSK system have a carrier tolerance of better than $\pm 10 \mathrm{~Hz}$, which at $433 \mathrm{MHz}$ (for example) would require a 23-part-per-billion oscillator. Although available, such oscillators are more expensive and consume more power and thus are unsuitable for our sensors.

Currently deployed spread-spectrum communication systems, like cell phones and GPS, precisely coordinate the transmission power of individual devices to maximize the sharing capacity of the channel; the coordination requires expensive central stations [16, 21]. In contrast, our wireless sensor network uses spread spectrum to reduce the cost of the sensors; therefore, precise coordination is undesirable because of its high cost. Implementing power control would significantly increase the cost of our wireless sensors because the coordination would require a bidirectional link, whereas the sensor itself requires only a simplex link. Including a receiver in the sensors would also significantly increase the power consumption. However, for static systems that will not be changed once installed, it is possible to adjust the output power of the sensors so that all the sensors will send approximately the same level of signal to the central station. Examples of systems that remain static are HVAC sensors for office buildings and sensors for process controls in factories.

The proposed CPSK technique, along with advanced coding schemes (Gold and Kasami), were employed for multiple-access network demonstration. Classical CDMA calculations are based on having a large set of different spread-spectrum codes to assign individually to each transmitter. Table 2 shows the number of available orthogonal codes using preferred pairs and their effective processing gains for the most popular spreading codes using a 1023 code length.

Table 2 The number of available orthogonal codes and their effective process gains for the most popular spreading codes using a 1023 code length.

\begin{tabular}{|l|l|l|}
\hline Code type & Number of codes & Effective process gain (dB) \\
\hline PN sequence & 60 & 24.211 \\
\hline Gold sequence & 1025 & 24.211 \\
\hline Kasami sequence (large set) & 32800 & 24.211 \\
\hline Kasami sequence (small set) & 32 & 30.370 \\
\hline
\end{tabular}

The data encoding was performed in software on a commercially available embedded computer to enable economical hardware and allow options for upgrading the system. Alternate configurations can be easily programmed for power-saving modes, periods between transmissions, and sensitivity. The programmable design also allows encryption options. The proposed improvement to the system is to add small repeaters to the network that could be used to improve the utility and reliability of the network without significantly increasing the cost of the sensors themselves. 
Typical data packets for building monitoring systems contain measurements of the temperature, humidity, and ambient light and are thus very small; furthermore, these parameters change slowly and thus need be reported only once every several minutes [5]. However, the overall data rate is significant because a large office building could contain hundreds of sensors. For a given amount of data capacity and range that a particular sensor network needs, there are two metrics that describe the spectrum efficiency of the system. The first metric is the bandwidth per data rate, or $\mathrm{Hz}$ per bit-per-second (Hz/bps). The second metric is the land area, e.g., square meters, that the system occupies. These two metrics are interrelated in that for a given geographical area, if a system is designed to occupy only half of the land area, the other half could be used by another system, doubling the capacity of the geographical area. Alternatively, these could be combined into a single metric of "area $\times$ bandwidth per bits-per-second." In this section, this metric is simplified by crediting the effective bandwidth by the ratio of reduced land area required.

There are two major methodologies for allowing simultaneous transmissions for the simple spreadspectrum transmitters. One is using the static power adjustment, as described above, and the other is the closer spacing allowed between neighboring systems compared with QPSK systems. Continuing with the closer spacing example, Figure 10 illustrates the minimum separation distance between neighboring systems that are sharing the same frequency.

For a standard QPSK system, the rule of thumb is that the signal requires an SNR of $6 \mathrm{~dB}$ (4:1 power). For base station $\mathrm{A}$ to receive a good signal from
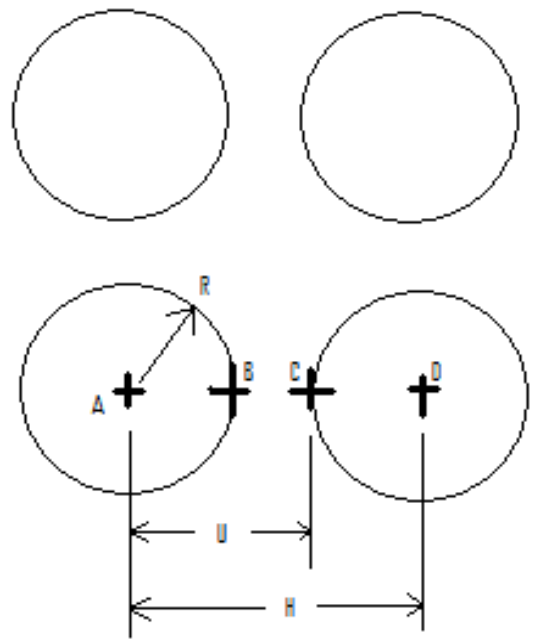

Figure 10 Illustration of node placement. transmitter B without being jammed by transmitter $\mathrm{C}$, distance $U$ is calculated by

$$
4=\frac{\frac{1}{R^{2}}}{\frac{1}{U^{2}}} \text { or } U=2 R ; \text { thus } H=3 R \text {. }
$$

Because of the processing gain provided by spread spectrum, the system will be more tolerant of the neighboring signal and the neighborhoods can thus be closer together. Given a process gain $G$ that rejects the neighbor signal, the spacing can now be calculated as

$$
4=\frac{\frac{1}{R^{2}}}{\frac{1}{G U^{2}}} \text { or } U=\frac{2 R}{\sqrt{G}} ; \text { thus } H=\frac{2 R}{\sqrt{G}}+R \text {. }
$$

In comparing QPSK with spread-spectrum systems, the goal is to increase the number of systems that can be operated in a given geographical area, i.e. reduce $H^{2}$. This area improvement ratio versus process gain, or spread-spectrum versus QPSK, can now be described by 


$$
\text { Area Ratio }=\frac{9}{\frac{4}{G}+\frac{4}{\sqrt{G}}+1} .
$$

For the 1023:1 spread-spectrum gain, $G=1023$ and the area ratio improvement is 8:1. This situation describes the neighboring systems to be overlapping or essentially co-located. For the building HVAC example, this is not possible. However, it still allows the neighboring systems to be next to each other, thus $H=2 R$ instead of $H=3 R$, which gives a 2.25:1 improvement.

For the static power adjustment methodology, the ideal situation would be for the installer to test the recently installed sensor and get a signal strength report from the base station. The sensor could then be adjusted to achieve the desired signal strength, and then the test and adjustment procedure repeated until the desired strength is obtained. A less rigorous method would be to install a low/medium/high version of the sensor based on approximate distance to the base station. Even if the signal levels are precisely adjusted, the environment will not necessarily remain completely static because of weather changes and movement of furniture and equipment. If the power mismatch is poor, the sensors will still get through but with a higher percentage of lost packets.

To determine the best possible number of simultaneous transmissions if the power levels are well matched, the SNR is calculated based on the number of other transmitters and the process gain. Using the rule of thumb that a digital signal requires an SNR of $6 \mathrm{~dB}$ (4:1 power) and using the $30 \mathrm{~dB}$ process gain (1000:1 power) from a 1023:1 spread-spectrum system, the number of simultaneous transmitters $N$ is described by

$$
6 d B=\frac{\text { Desired } T X \text { Power }}{N *(\text { Neighbor } T X \text { Power }) / 30 d B}
$$

This gives $N=250$. Combining the 2.25:1 area improvement computed in Eq. (3) with the additional number of transmitters just described, the overall improvement would be 562:1. Since the extra bandwidth required for spread spectrum over QPSK in this case is 102.3:1, the 562:1 factor will recover all the extra bandwidth consumed and even provide a further benefit. However, there are several reasons why this ideal case is not approachable. There are several practical limitations, such as the ability to match the transmitter power levels, but there are fundamental reasons why the $30 \mathrm{~dB}$ process gain cannot be reliably achieved.

Available literature in the spread-spectrum field does not describe the general case where the different signals are displaced in time and the signals have various CPSK modulations. Coordinated spreadspectrum transmitters that have their CPSK start-stop boundaries begin at the same time would still obey

the classical cross-correlation characteristics within these boundaries, but generalized systems such as our sensors would not have this coordination. Because CPSK performance for uncoordinated transmitters is not as well analyzed in the available literature, it is necessary to perform simulations for these systems to determine how many simultaneous transmitters the system can reliably support. Furthermore, the simulations should study situations in which the transmitters are not well matched in power, situations in which there are potentially other interfering systems in the neighborhood, and whether there are a sufficient number of spread-spectrum codes available to support systems of this size. The answers to all these concerns are addressed in Section 4.4.

\subsection{ORNL CPSK SIGNALING TRANSCEIVER}

\subsubsection{Fundamentals of Gold and Kasami CPSK}

This section presents the fundamentals of the implemented Gold and Kasami CPSK signaling transceiver, on which data encoding is performed in software on a commercially available embedded computer to enable economical hardware and allow options for upgrading the system. Alternate configurations can be easily programmed for power saving modes, periods between transmissions, and sensitivity. 
The proposed communication scheme is based on frequency modulation (FM), which is designed to fit into the P25 Land-Mobile infrastructure. It does not have the same encoding as P25, so it is not compatible with it; but it will have the same FM deviation and symbols-per-second rate and thus consume the same spectrum resources. Since P25 is encrypted, a different P25 user could regard our signal as another P25 signal for which the encryption keys are unknown. It would also be possible to contrive a way for one of our packets to be fed into a P25 packet. It would be contrived to resemble one of our original packets when it went over the air. There are several reasons why operating in the Land-Mobile band would be beneficial to some customers. One is that several entities, such as police, railroads, and utilities, already use these bands. They could use them for typical voice operations during the day and use the same frequencies for sensors during the night. Their frequencies are considered licensed "private channels," so they would not have to worry about interference as they would with unlicensed bands. Land-Mobile users also have a need for long transmission distances that traditional unlicensed band radios (such as WiFi) do not serve. There are many Land-Mobile bands available, such as $30-50 \mathrm{MHz}$, 150--174 MHz, 406-420 MHz, 450-512 MHz, and around $950 \mathrm{MHz}$. These represent a lot of bandwidth; and as many users have switched to cell phones, there should be available channels in these bands.

There are some subtle differences in designing a receiver for AM or for FM besides the obvious detector. AM systems require an automatic gain control (AGC) to get sufficient strength at the detector yet not too much signal where the signal clips. However, FM systems still work even if the signal is clipped because the frequency of the clipped signal is still there. Therefore, most FM radios eliminate the AGC, not only to reduce cost but also because the clipping "clips off" some of the noise and improves the radio sensitivity. Clipping also enhances the FM capture effect to reduce interference from other transmitters. For our spread-spectrum case, our signal will be below the noise level, so we must retain the AGC even if we are using FM. This also means that we will not be able to get the full $30 \mathrm{~dB}$ processing gain advantage for our FM version of the system.

The ORNL wireless tag sensors have progressively improved from spread-spectrum codes based on maximal length sequences (MLS) to Gold codes and finally Kasami codes. We have settled on using the Kasami version from now on, with the further option of having more than one Kasami packet after the preamble. The baseline system now has one 1023-bit-length preamble based on a Gold code, followed by one or more 1023-bit-length Kasami codes for the data packet. Information is encoded into the spreadspectrum sequence using the CPSK method, which involves pre-loading the shift registers with the data to be transmitted. The code generator will still send the same code when loaded with different data, but it will be shifted down in time.

The CPSK transmitter consists of two standard linear feedback shift registers (LFSRs) that generate the Gold coded spread-spectrum sequence. An LFSR is the heart of any digital system that relies on pseudorandom bit sequences [21], with applications ranging from cryptography and bit-error-rate measurements, to wireless communication systems employing spread-spectrum or CDMA techniques. A schematic diagram of the implemented Gold CPSK transmitter is illustrated in Fig. 9. It consists of two sets of 10 LFSR systems with an XOR gate. There are pairs of feedback tap combinations for each of the LFSR systems that will generate a new Gold sequence that does not repeat itself within the sequence and has good autocorrelation characteristics. For Gold codes, if the two MLS halves are shifted by the same amount, then we will get the same Gold code shifted in time. If the halves are shifted by a different amount, we will additionally have a different Gold code. For our purposes, we are using different Gold codes to indicate different customers; yet we do not want a particular Gold code to be shifted because the subsequent data packet is synchronized to the Gold code timing. A key point to note is that the two seed values can be chosen independently from each other; thus, we can encode 20 bits of data in this 1023-bitlong sequence. For this system to work, a transmission should consist of an initial blank sequence followed by one or more of the data sequences. The blank sequence is needed as a time reference to show when the start time of the data sequence is. The blank sequence does not have to be a Gold code but can be a simpler LFSR code. To ensure proper operation, one MLS half is always assigned all "ones" while 
the other MLS preload indicates the customer number. The implemented Gold CPSK prototype transmitter is illustrated in Figure 12. The schematic diagram of the implemented Kasami CPSK transmitter is illustrated in Figure 13, in which the Kasami data packet changes the loaded data corresponding to the data to be sent. The implemented CPSK prototype transmitter is shown in Figure 14 and Figure 15, and its specifications are summarized in Table 3.

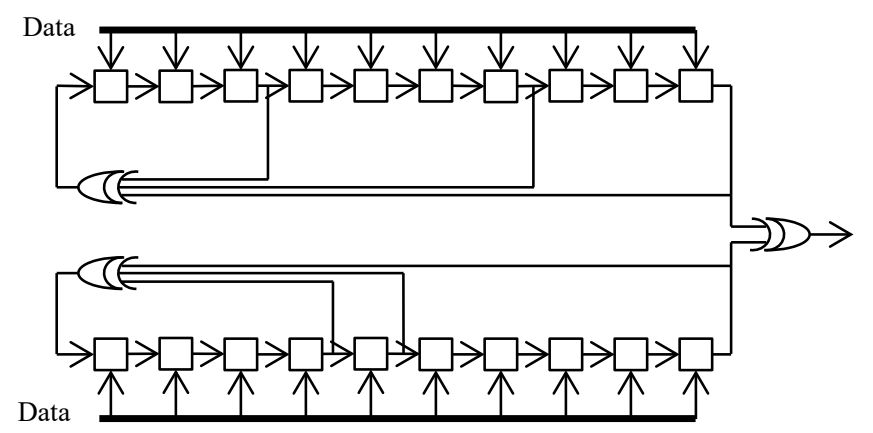

Figure 11 Schematic diagram of the implemented Gold CPSK transmitter.

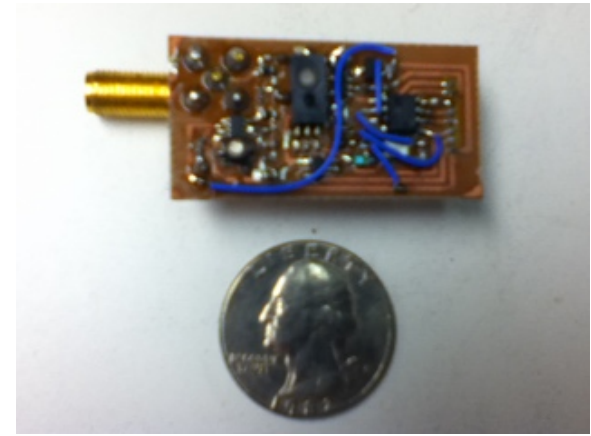

Figure 12 The implemented Gold CPSK transmitter prototype.

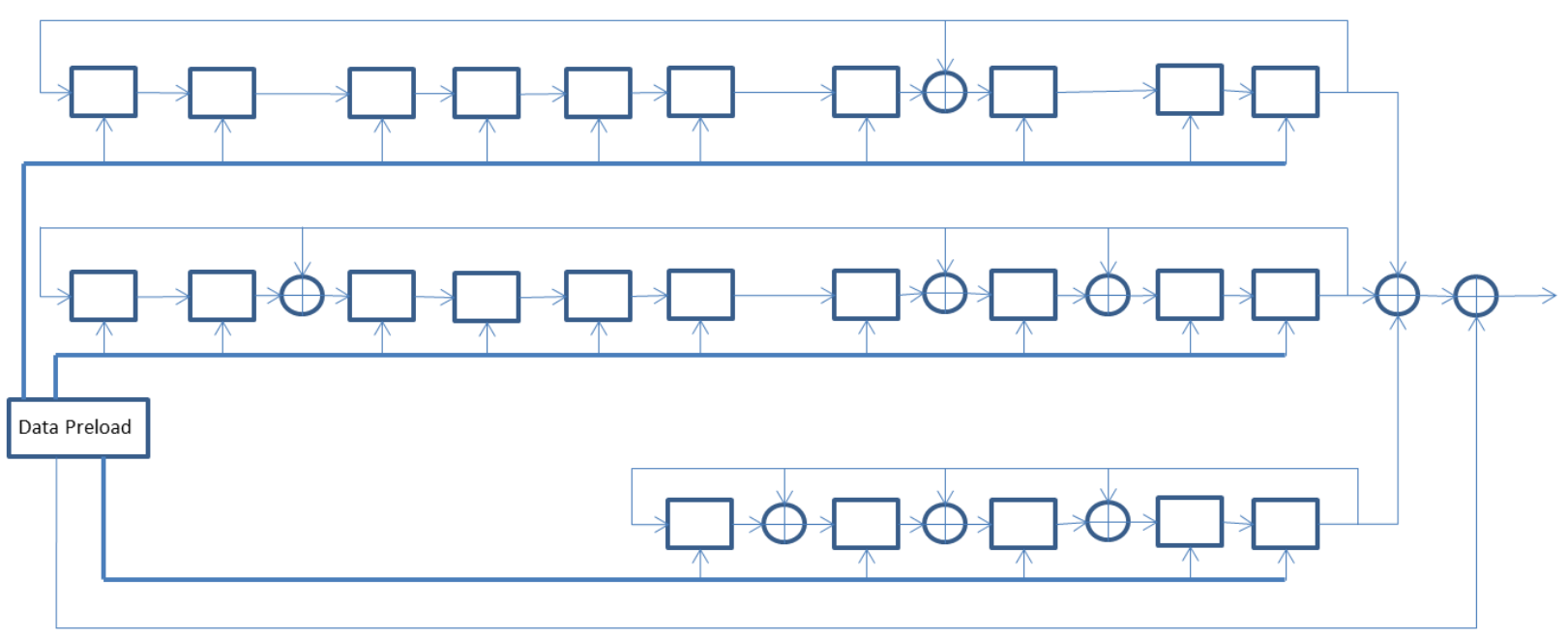

Figure 13 The implemented Kasami CPSK transmitter prototype. 


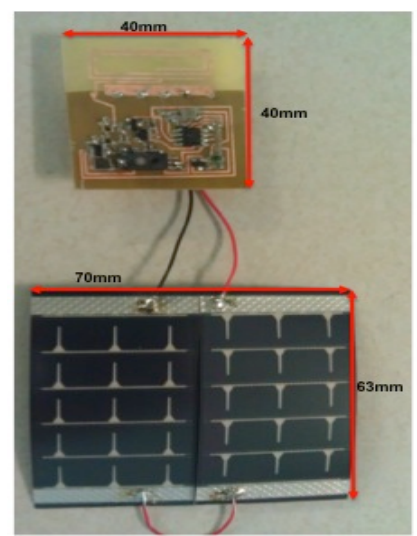

Figure 14 The implemented CPSK prototype transmitter: fully functional prototype on FR4.

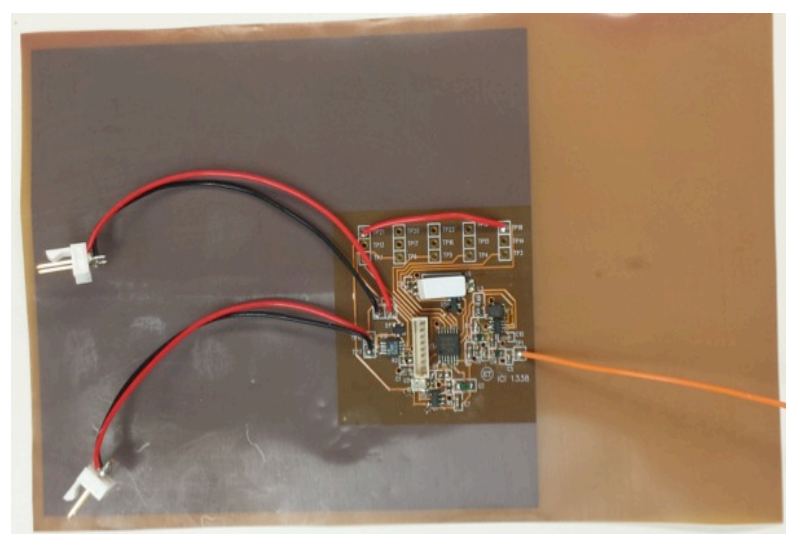

Figure 15 The implemented CPSK prototype transmitter: fully functional prototype on thin film.

Table 3 Specifications of the implemented CPSK transmitter prototype.

\begin{tabular}{|c|c|}
\hline Transmitter frequency & $433.92 \mathrm{MHz}$ \\
\hline Transmitter power & $+7 \mathrm{dBm}$ \\
\hline Receiver sensitivity & $-145 \mathrm{dBm}$ \\
\hline Chip rate & $2000 \mathrm{bps}$ \\
\hline Data rate & $40 \mathrm{bps}$ \\
\hline
\end{tabular}

On the receiver side, the signal is decoded by looking for a pattern that looks like the expected transmitted signal. This is done by conducting a one-to-one bit comparison between the last received 1023 bits and the expected pattern. In looking for the blank sequence at the beginning, there is one expected pattern to look for. After the blank pattern is detected, the next 1023 bits are recorded and data patterns are sought. However, because of the varying data, there is not just one pattern to look for but a whole family of patterns, with each family member pattern being a possible data packet. Unfortunately, our method of finding which family member packet was sent is to perform an exhaustive search, as illustrated in Figure 16. This means that for 20 bits of data, there are about one million possibilities for the seed values, and thus we need to compare one million test case patterns against the pattern that was received. Although this sounds unreasonably difficult, a standard PC laptop can perform this search in about 10 seconds with a moderately sized C program. Using a field programmable gate array (FPGA), the search can be performed in 10 milliseconds. Since the HVAC application requires a sensor reading only once a minute at most, these performance results are acceptable. 


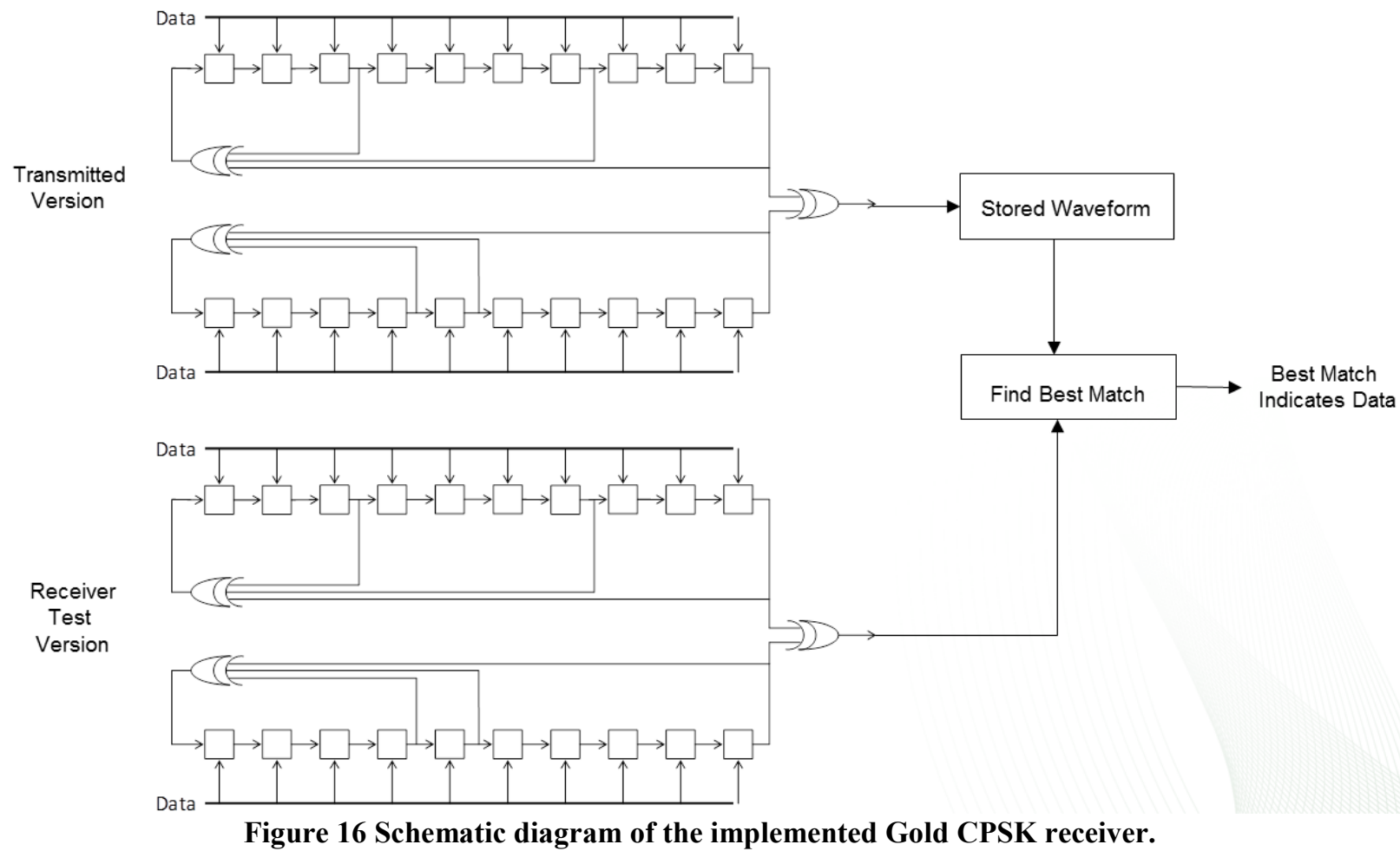

Correspondingly, the Kasami receiver determines what the preloaded data are by conducting a trial correlation for each data stream possibility, as illustrated in Figure 17. Since the waveform is stored, this process does not need to run in real time. There will be 25 bits of data, so there will be $67,108,864$ possibilities to try. The input data are four times oversampled, so for each possibility there will be 4092 additions/subtractions, for a total of 274,609,471,488 operations. The latest FPGA design implementation can perform these computations in about 0.5 seconds. If there is more than one data frame, the total decode time will be 0.5 seconds per frame. An important question is whether there are more efficient techniques than doing a full exhaustive search. There have been a few trials in the literature regarding some limited cases in which a more efficient technique is possible, but to date we have not found anything that works other than an exhaustive search for the generalized case.

Currently, we have two versions of the spread-spectrum CPSK system, the first of which is unencrypted with one preamble frame and one data frame. The other is encrypted using the xTea code and has one preamble frame and three data frames. The transmitters are based on Microchip PIC16LF1823 computers that are programmed in $\mathrm{C}$. The $\mathrm{C}$ program does all the data acquisition, data formatting, encryption, spread-spectrum calculations, and final bit-banging of the transmitter chip. At the receiver, a custom ORNL radio receives the signal, converts it to baseband, and then sends it to the FPGA. The FPGA correlates this signal to determine the received data that are decoded in a $\mathrm{C}$ program on the Zynq processor. The MLS calculations are done using the Galois method. For the preamble, the first LFSR is always $0 \times 3 \mathrm{FF}$, and the second LFSR is 1 to $0 \times 3 \mathrm{FF}$, corresponding to the customer number. In searching for the data, we note that we should contrive the data so that none of these states will be zero, because this would lock the MLS to always be zero. There is one technical modification-inverting the entire data sequence via XOR to indicate the least significant bit (LSB) of the address to give a total of 26 bits. Figure 55 shows the implemented CPSK prototype receiver. In this design, the tentative 26-bit data format consists of 7 address bits for transmitter identification, 7 bits for temperature, 5 bits for humidity, 1 bit for lighting, and 6 bits for parity check. 
The xTea packets are 64 bits in length; this is much more than we need, although it allows us to thoroughly send the data at their full resolution and allows more robust parity checking. To deliver 64 bits, we will need three 25-bit frames. To eliminate the 0 problem with the MLS starting point, we will not use the LSB of each MLS starting state but instead put a "1" there. That will leave us 22 bits for the first frame, 22 bits for the second, and 20 bits for the third. The xTea standard reminds us to always add some dithering to data to prevent the encrypted stream from looking the same if the input data, such as temperature, is a steady value. An 8-bit counter was added for this purpose. Also, xTea does not include any parity checking; but since we can expect the encoded stream to look dramatically different if we lose a single bit, then looking for a special number in the data can serve as this parity check. The 10-bit number was added for this purpose. The sensor tag address was expanded to 12 bits, and the maximum resolutions for the temperature and humidity from the sensor chip are sent, which are 14 and 12 bits, respectively. Finally, the light intensity resolution is 8 bits worth. The 64-bit data consist of two 32-bit words.

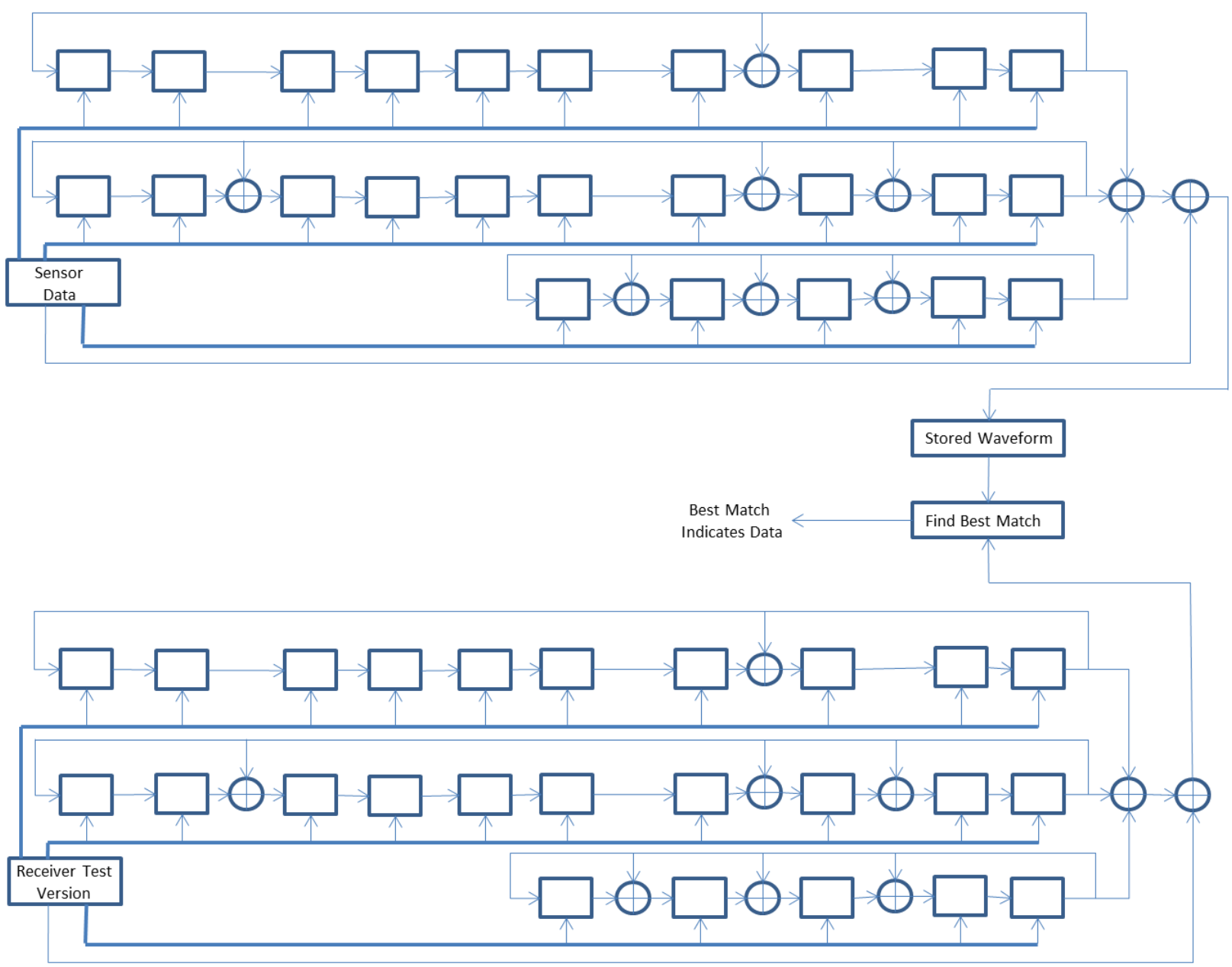

Figure 17 Schematic diagram of the implemented Kasami CPSK receiver. 


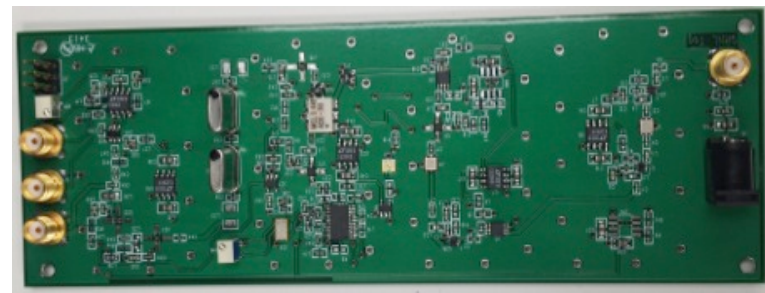

Figure 18 The implemented CPSK prototype of the wireless receiver.

In summary, the implemented low-power CPSK spread-spectrum transceiver has the following features:

- Unidirectional

- TDMA style packet bursts

- 5 milliwatt output power

- 1023-bit length Gold code

- 2048 bits per second chipping rate

- Data of 20 bits to preload registers

- Spread-spectrum length of 1023 bits to deliver the 20 bits (51:1 expansion) - a $30 \mathrm{~dB}$ gain

- More than 1 million "orthogonal" shifted Gold codes generated

- Neighboring cells can be closer together without requiring power control

- $1 \mathrm{~W}$ performance from a $1 \mathrm{~mW}$ transmitter (battery consumption reduced by a factor of 10 compared with a regularly modulated system of the same range)

- Smaller battery, low-current operation

- Relaxed tolerance $2 \mathrm{KHz}$ vs. $40 \mathrm{~Hz}$ for $40 \mathrm{bps}$

- Normal frequency accuracy requirements

- Bandwidth recovery-CDMA

- $-144 \mathrm{dBm}$ receiver sensitivity

- 0.6 mile demonstrated free space range

- Can operate once a minute, 24 hours a day from a 3 by 4 inch solar cell illuminated with 8 hours of office lighting

The initial version of the tag receiver system was implemented on a Windows PC; and although it performed accurately, it was very slow. An effort then began to implement the receiver on an FPGA. Although doing so took a lot of time and effort, the result is now a viable product that decodes the data in near real time and is faster than the PC by a factor of more than 1000. The Zynq FPGA used is a fairly expensive part, and some potential customers have expressed concern about the cost. However, if the technique becomes a stable industry standard, then the design could be implemented on a less expensive ASIC or even a full custom mass-produced chip. Achieving this speedup on the FPGA required trying all the pipelining and parallelizing tricks that were available.

The latest version is nearly the final version because it has consumed almost all of the FPGA resources but also implemented essentially all the needed features. As FPGA technology improves so that more computational power becomes available, the method can be easily extended to make decoding time faster or reduce system costs for a given rate.

\subsubsection{Information Flows and Methodology}

This section presents the information flows and methodology for pipelining and parallelizing the algorithms. Note that the architecture sets up the packet capturing and data correlation designs to be controlled and run independently. The packet capture design detects the preamble and stores the following data frame in a ring queue. Afterward, the data correlator analyzes the frame in the queue. This allows new packets to be received even when the FGPA is busy decoding the previous packet. Packets 
will not be dropped provided that they do not arrive faster than one frame per 0.5 second on average. This feature is also what allows us to receive packets that have multiple frames, such as for the xTea.

Several parameters for the system need to be set from the $\mathrm{C}$ program on the Linux side of the Zynq. After a general reset is performed, the first controls are for setting the preamble and frame-saving parameters, such as customer number, thresholding value (squelch), and number of frames to save per packet. Next are controls for inserting and latching simulated data or gathering real data with the specified chip rate $(2,048 \ldots 32,768)$. When a new packet is received, the data frame is stored in the queue and the corresponding memory block number is presented to the $\mathrm{C}$ program. When the $\mathrm{C}$ program sees that the block number has incremented, it then tells the correlator design to decode this block. Note that the block being decoded can be several blocks behind the current block being stored. The $\mathrm{C}$ program can read several bits that indicate that the decoding process is progressing or is finished. When it is finished, the program reads the starting spread-spectrum states that correspond to the best correlation. The magnitude of the correlation value is also read. The $\mathrm{C}$ program then determines the actual data from these values. The diagram of the overall design is illustrated in Figure 19.

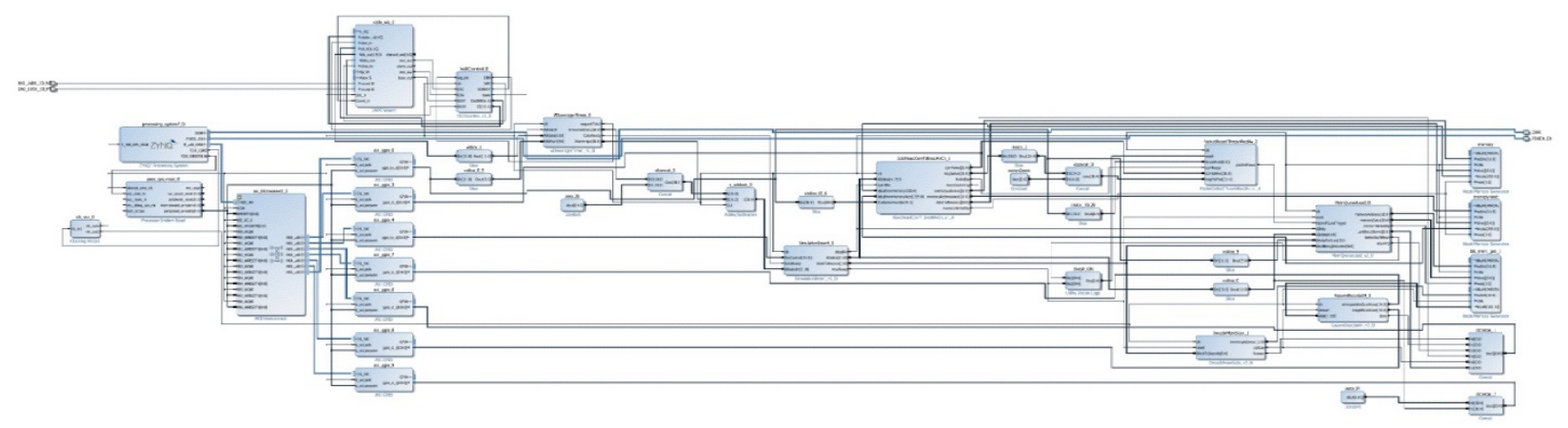

Figure 19 Diagram for the overall FPGA design. 
One important detail is the individual bits for the control functions. These bits are illustrated in Figure 20.

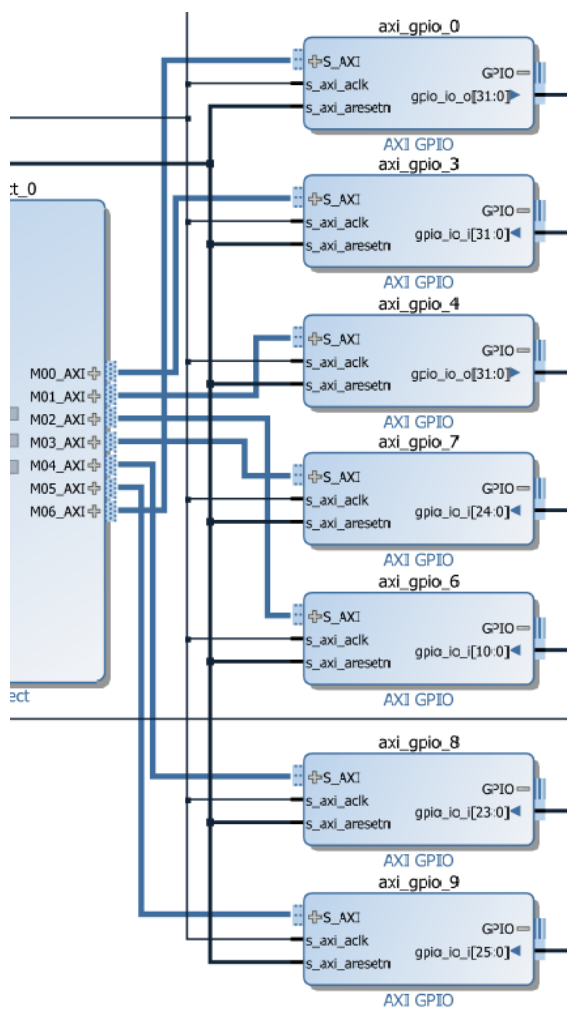

Figure 20 Zoomed diagram for the individual bits of the control functions.

\subsubsection{Custom Blocks}

This section briefly describes the custom blocks. There is a VHDL file for each block that has the same name as the block. The first part is the standard Vivado system for connecting the Zynq Linux processor to the FPGA logic (illustrated in Figure 21). It is straightforward, with the addition of the Xilink ADC (XADC) block for the $\mathrm{A} / \mathrm{D}$ and a clock wizard to provide a $50 \mathrm{MHz}$ clock for most of the system, as well as a $100 \mathrm{MHz}$ clock to run the high-speed correlator. Information crosses the clock boundary at only two locations: the ring queue memory and the AXI general-purpose input-output (GPIO). The dual port memory has separate clocks, so it accommodates this easily. The GPIO should not be a problem because a correlation starts when the BlockToDecode changes, and it is okay if this value settles out over the next clock cycle. At the end of the correlation, the $\mathrm{C}$ program looks for only one bit to change; then it reads the correlation values a few clocks later after things have settled. The next blocks are for controlling the analog-digital converter (ADC), as illustrated in Figure 22. The block XADCcontrol does the low-level handshaking for the XADC and sets it to read the pins for channel 15. There are several parameters that need to be set inside the XADC, such as the clock frequency, single channel mode, and triggering mode. $\mathrm{XADC}$ control responds to requests from ADaveragerTimer and tells it when the reading is ready. ADaveragerTimer is an interesting block that not only sets the data collection rate depending on the chip rate but also does some low-pass filtering and provides a very-low-frequency filtered signal for DC estimation purposes. The block always calls the A/D at 328,947 (50e $/ 152)$ SPS and averages these values to get an output sampling rate that is four times the chip rate. The averaging is a square window type; in other words, it simply totals up all the input samples starting just after the last output is sent out. To make test points available, the DC value is subtracted from the smoothed signal outside the block to give an AC signal with no DC component. 


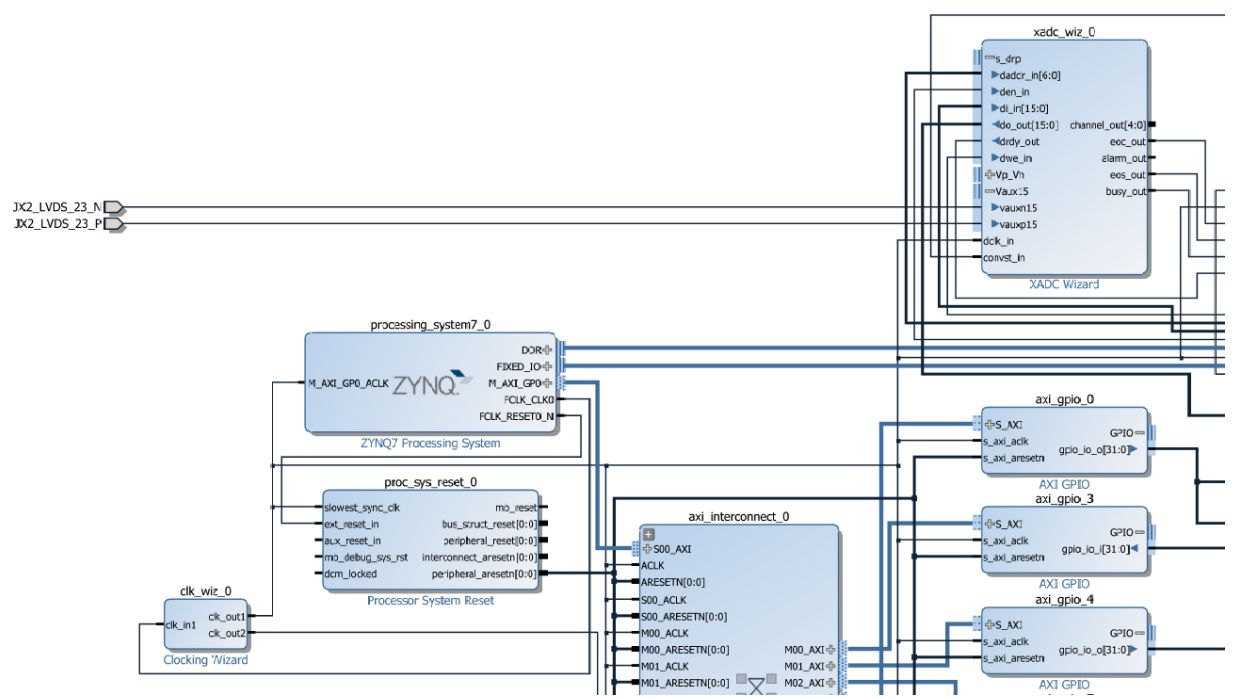

Figure 21 Zoomed diagram for the standard Vivado system that connects the Zynq Linux processor to the FPGA logic.

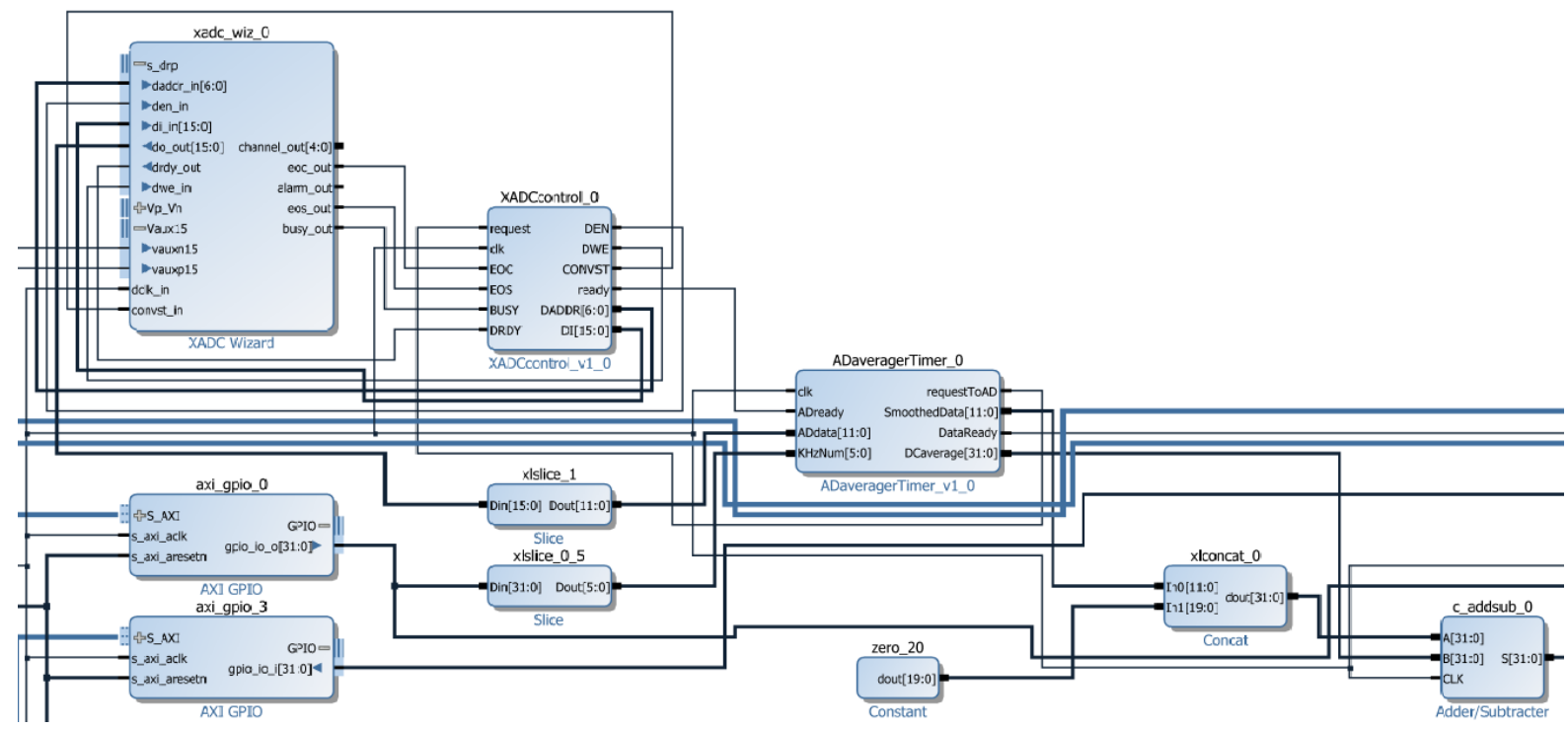

Figure 22 Zoomed diagram for control of the ADC. 
The block SimulationInsert intercepts the input signal path to put a simulation instead (Figure 23). It also passes through the BlockToDecode information. The simulation features can probably be removed later.

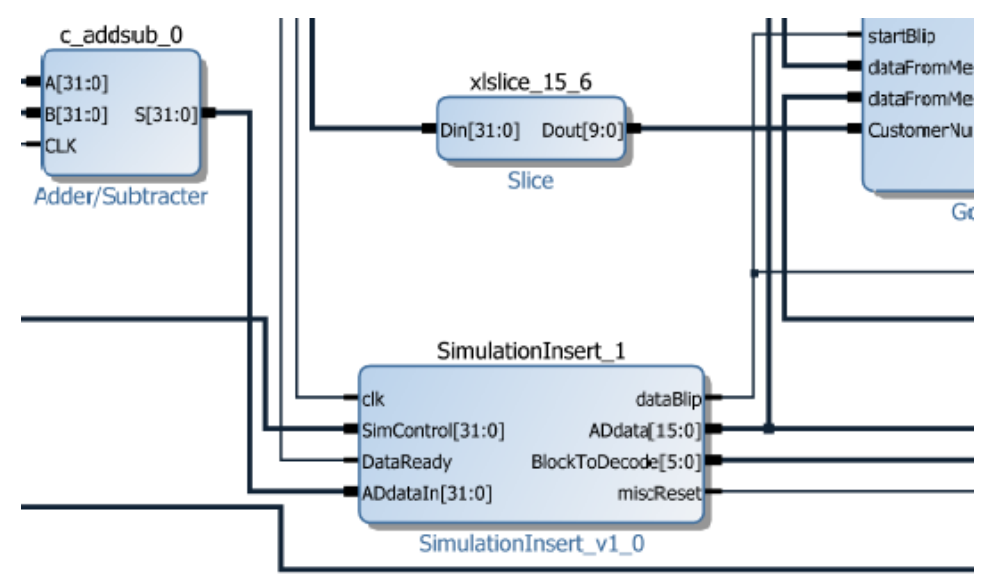

Figure 23 Zoomed diagram for the SimulationInsert block.

The function of GoldHeadCorrT16rectAvCn is to take the customer number and generate the corresponding Gold code sequence, then use this sequence to correlate the last $4092 \mathrm{~A} / \mathrm{D}$ samples (Figure 24). When a packet comes in, the correlation output spikes up at the end of the preamble. Because the spike output is proportional to the overall input signal strength, this block also puts out an indication of the signal strength. This indication is a rectangular average of the last 4092 samples-hence "rectAv" in the module name. These signals go to the block PacketDetectThreshRectAv, which detects when the correlation is greater than the value of the threshold multiplier times the average value.

PacketDetectThreshRectAv is careful to note that the correlation is still increasing so it declares a detection when the correlation starts going back down. These two blocks are what determine the radio sensitivity, so it is very important that they be implemented accurately. A key discovery was that determining the overall magnitude by averaging the signal with an infinite impulse response (IIR) -style low-pass filter was not sufficient because IIR filters do not filter out transients well.

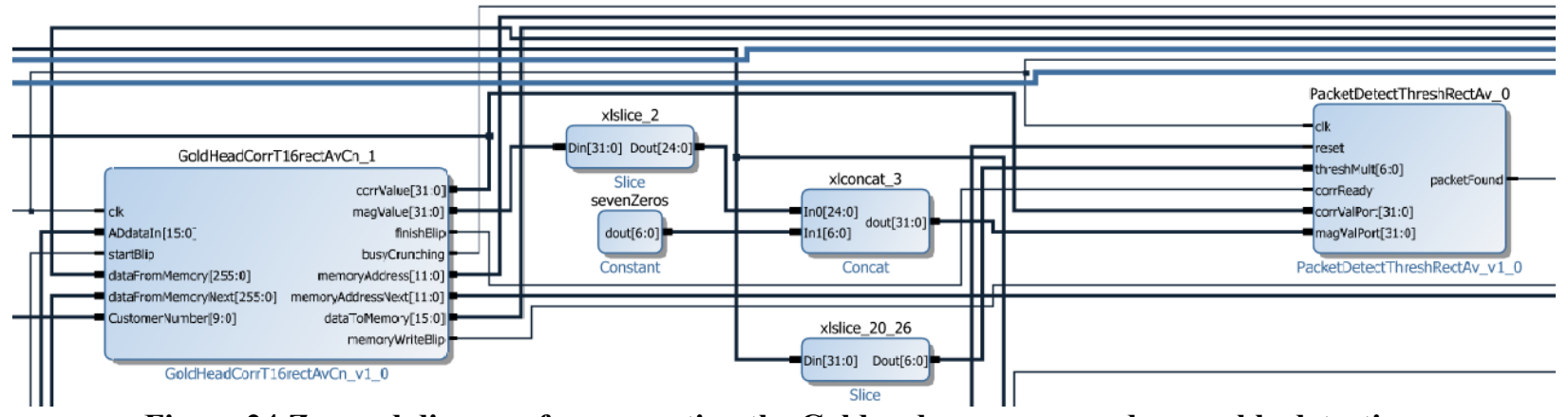

Figure 24 Zoomed diagram for generating the Gold code sequence and preamble detection.

What really makes GoldHeadCorrT16rectAvCn special is its parallel processing. For every sample that comes in, the correlator must reproduce the Gold code and use it to perform 4092 additions or subtractions. At the highest chip rate, these must be performed in $1 /(32768 * 4)$ seconds or 7.6 microseconds. To enable this speed in real time, we must parallelize the calculation by at least a factor of 16-hence "T16" in the block name. The basic plan is to take the last A/D sample and store it in one of the 
Xilinx block memories, then read back the last 4092 samples from the memory and correlate it with the regenerated Gold code stream. Storing the Gold code stream in memory instead of regenerating it was considered, but at this time we considered the regenerator code to be simple and more compact than memory interface code.

The Xilinx memory blocks have convenient dual ports at which we can input data at the A/D data width (16 bits) during the sampling phase, yet pull out 16 consecutive samples ( 256 bits worth) during the correlation phase. The 256 bits are read back using the same address as the input data but with the lower 4 address bits removed. Thus, the 256 bits will not start evenly with the last sample. Many workarounds were investigated, and we settled on having two duplicate memories that stored the same A/D data; yet, during read-back we bumped the second memory address by 1 so that we could read 512 bits or 32 samples. The correlator could then sort out the desired 16 samples from within the 32 sample window. The key reason this method was considered the best was that it simplifies the code, and a large amount of block memory is reserved on the Zynq anyway.

After the packet preamble is detected, the next step is to capture the data packets. The MemQueueLoad block does this, as illustrated in Figure 25.

Similar to GoldHeadCorrT16rectAvCn, MemQueueLoad stores 1023 bits worth of A/D data in a Xilinx memory block; but since we now know where the bit edge is, we can sum up the corresponding 4 consecutive samples for a bit to store 1023 values instead of 4092. One or more 1023-value frames (or blocks) are saved according to the parameter BlocksPerGrab, and the number of the last block is posted with LastBlockStored. The input BlockBeingDecoded is used to have the system hold off on grabbing more data if the decoding system gets far behind. Up to 63 frames can be stored in the memory at a given time, which corresponds to 63 old-style packets or 21 xTea packets. These numbers represent how far the decoder can get behind, because normally the ring queue just wraps around.

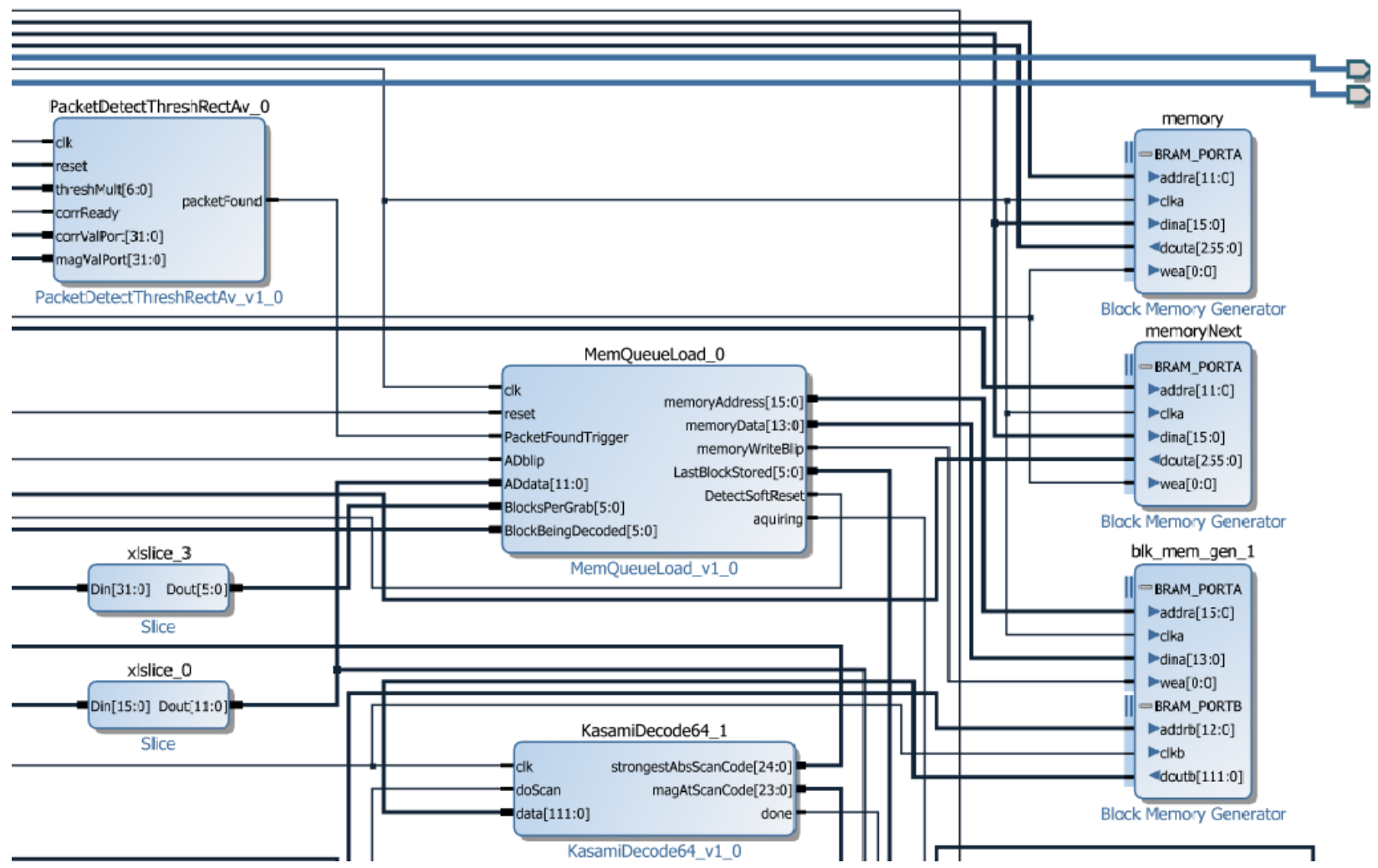

Figure 25 Zoomed diagram for detecting the data packets. 
Kasami decoding is the most time-consuming portion of the system, so here is where we see the most parallelization efforts. There are several levels of parallelization, and some unusual coding arrangements were made to reduce the number of additions and subtractions to save code space. New methods were developed to automatically increase the parallelization, so now we simply increase the amount of parallelization until just before we run out of FPGA space. The Kasami correlation started out being similar to the GoldHead correlation. The first modification was to modify the design to generate Kasami codes. The next was to calculate a correlation on the 1023 data values for each possible starting state value for the Kasami codes. This was equivalent to exhaustively checking all the possible values with which the transmitting sensor tag could start for the three LFSR variables. As each possible correlation was calculated, it was compared with the current best correlation to see if this new correlation was the strongest. The LFSR values corresponding to the strongest correlation were the actual data.

Next, we wanted to perform this task faster. The first improvement was to double the clock speed to $100 \mathrm{MHz}$. The next was to sum up all the A/D samples corresponding to a particular bit; this was done in MemQueueLoad. The next task was to calculate as many churns of the Kasami code as we could in a single clock tick. Currently, we are churning it 8 times because previous attempts for the next possibility, 16 , could not make time. The outputs from these eight churns are saved to be used on the next clock cycle, which is a pipelining method of parallelization. An eight-sample accumulation of the A/D data (112 bits) are read in on each clock cycle, and the corresponding eight outputs from the Kasami generator are used for correlating this portion of the data. The next parallelization effort was based on multiple copies of singleCorrKasamiOcto working on separate portions of the LFSR state possibilities. Currently, we have 64 copies of singleCorrKasamiOcto running in parallel-hence the "64" in KasamiDecode64. Note that all of these 64 copies are working with the same A/D values at a given time but using different Kasami state possibilities.

Finally, the outputs from those 64 correlators needed to be compared to select the best performer. At this point, we designed a set of simple daisy-chain connected shift registers that serially fed the results to a final routine that recorded the strongest correlation and its corresponding LFSR state values. This is a slower speed concept, but it only needed one greater-than $(>)$ design and it took only 64 clock cycles. Greater-than designs take up a significant amount of space, so it was important to minimize them. The blocks that implement these functions are illustrated in Figure 26.

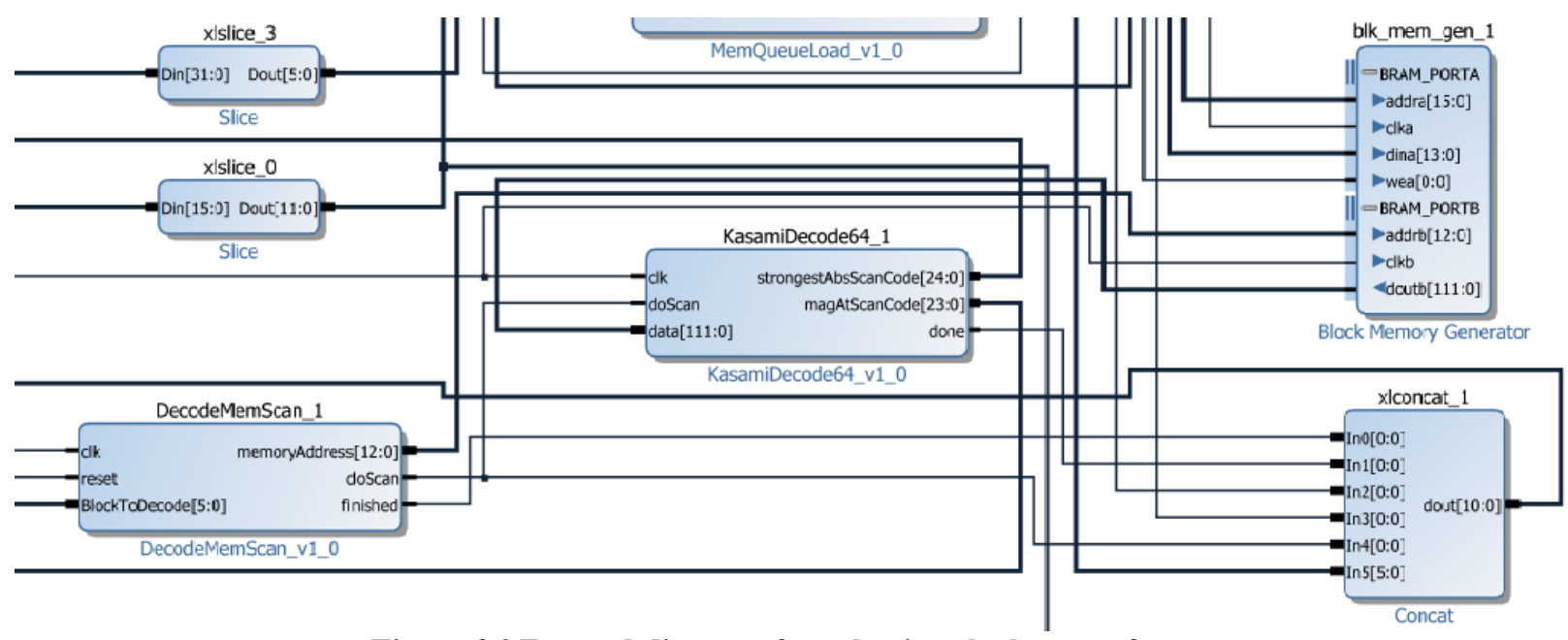

Figure 26 Zoomed diagram for selecting the best performer.

DecodeMemScan starts the process by seeing that the BlockToDecode value has changed. It then tells KasamiDecode64 that the process is starting by setting the doScan bit. DecodeMemScan keeps addressing 
the memory to get it to play back data for KasamiDecode64 to use. There are $2^{25}$ or $33,554,432$ Kasami state possibilities, but we reduced this number by 32768 to get 33,521,664 because of the non-usable zero states at the beginning. And since we had 64 correlators operating in parallel, DecodeMemScan only needed to do 523,776 playbacks of the frame A/D values. There are 63 frame storage possibilities in the memory, and DecodeMemScan addresses the appropriate one based on the BlockToDecode value.

Although AES is the world standard for encryption, AES proved to require too many resources to fit on our current transmitters. So we employed xTea, recommended by Microchip. The xTea code barely fits on the transmitter. The packet size for xTea is 64 bits, which is more than are needed. Implementing xTea required sending twice the number of bits as in our original system, so the battery life was reduced by two. The error rate for the larger payload seemed to be higher. The receiver could then accommodate longer packets, so we could transmit more payloads if necessary, such as $2 * 64=128$ bits.

Figure 27 and Figure 28 show the latest schematic diagrams for the transmitter and receiver designs.

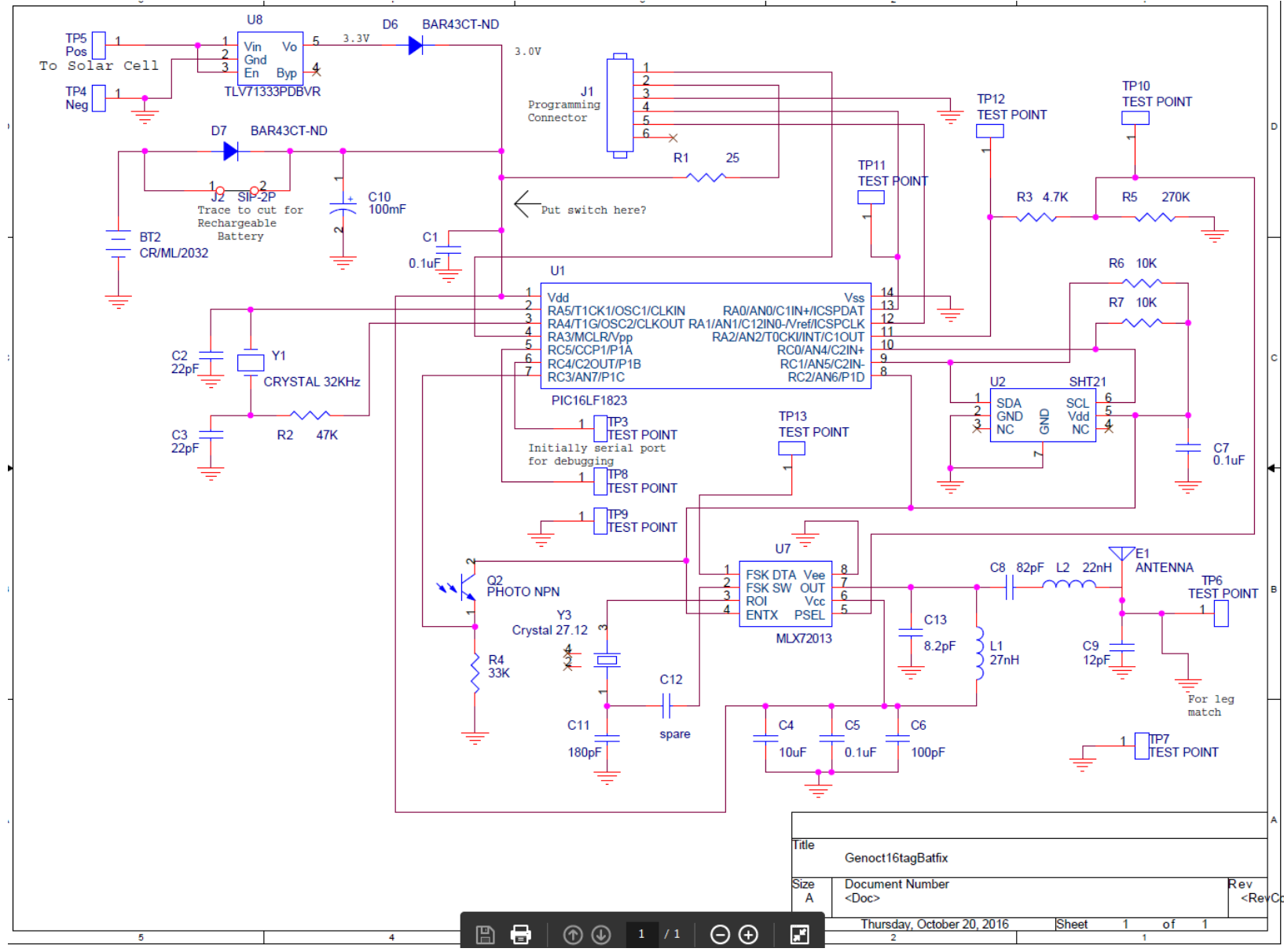

Figure 27 Schematic of the transmitter.

The implemented CPSK spread-spectrum transceivers can be deployed in buildings using the extendedstar topology (sometimes referred to as Star-Tree) communication network [22]. The star topology connects all nodes to a central point of concentration; this point is usually called a hub. The star-tree topology links individual stars together by linking the hubs to a central hub that extends the length of the network as illustrated in Figure 29. We recommend using this structure topology since it maximizes the transmission coverage area under a limited number of Gold/Kasami codes and transmission power. 


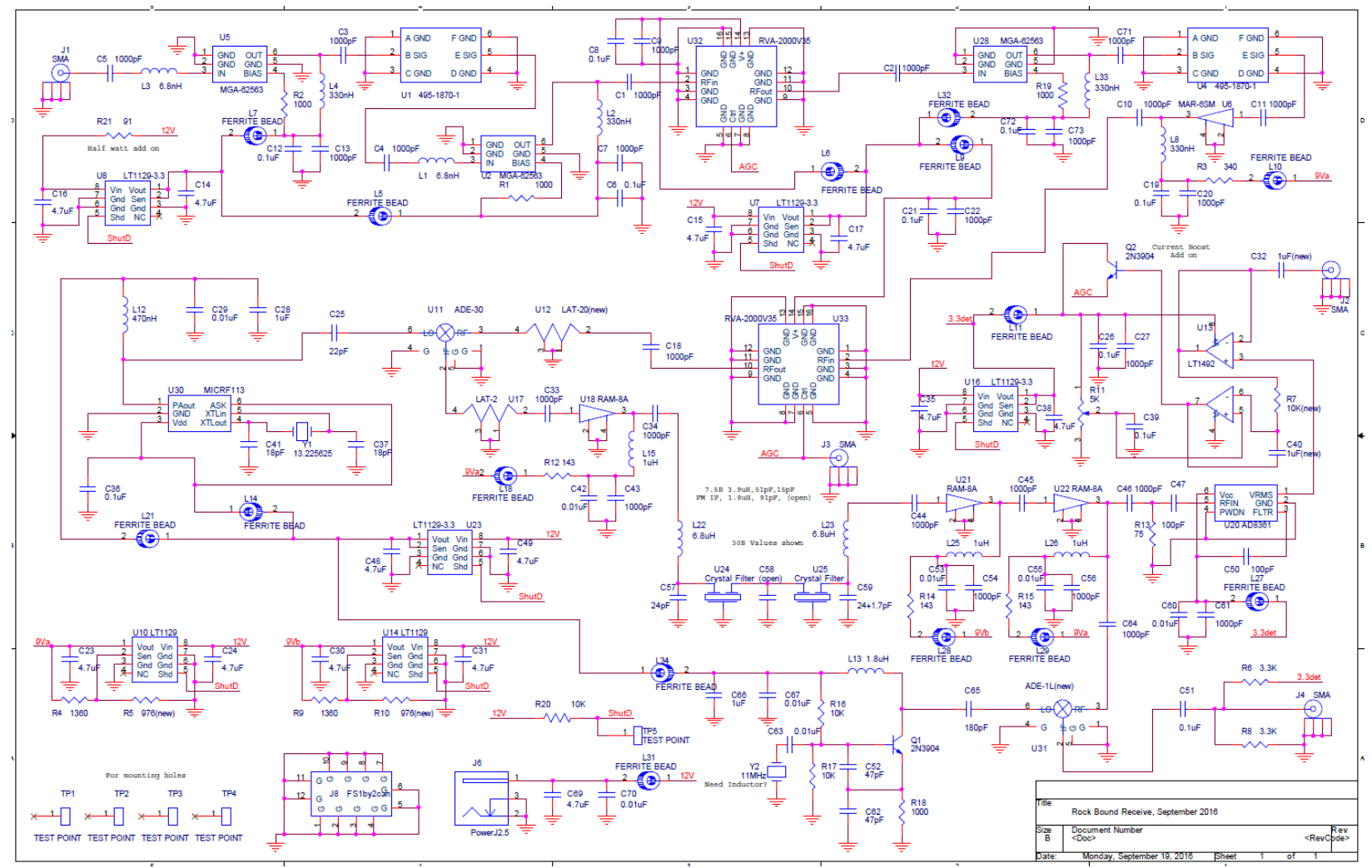

Figure 28 Schematic of the receiver.

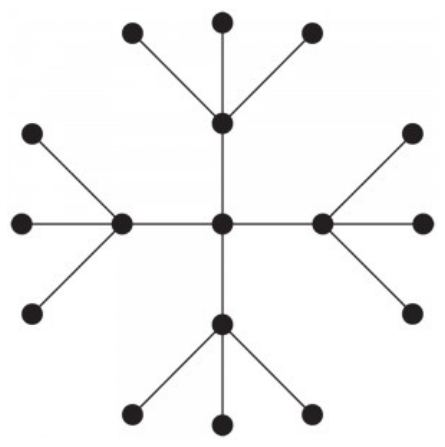

Figure 29 The recommended star-tree topology for sensor deployment.

In the star-tree topology, each node is connected to a central hub with its own communication link. If one link fails, then only one device that is connected through that link will be affected, while the rest of the network will remain operational. This topology has many advantages - such as the ease of installing it, detecting faults, and removing parts - and it causes no disruptions to the network through the connection or removal of devices. Its disadvantage is that if the hub or concentrator fails, all nodes attached to it are disabled [22].

In our design, the nodes represent the sensor transmitters and the hubs represent the central receivers. Each receiver hub has a different LFSR feedback tap combination (the g's in Fig. 23 and Fig. 24) so that each receiver has its own set of Gold/Kasami codes. Since each transmitter within a hub can send any of the shifted Gold/Kasami codes, the multiple access capability within one hub does not depend on CDMA but on a special type of time division multiple access (TDMA), which is a random multiple access scheme based on asynchronous TDMA. Pure TDMA won't work well in our application because the timing clocks for transmitters are not synchronized and usually drift after some time; the drift leads to continuous 
collisions among transmitters in the same hub. The multiple access capability among different hubs depends on CDMA because LFSR different tap combinations are employed among different hubs. The design parameters of interest are number of hubs, maximum number of nodes per hub, and coverage area per hub. The maximum number of hubs is determined by the maximum number of feedback tap combinations for Gold/Kasami codes; for example, it is 8 tap combinations in the case of the 023-bit length Gold code used in our design. The maximum number of transmitter nodes per hub is determined by the acceptable success rate for the employed multiple access scheme. And the coverage area per hub is determined by the maximum permissible transmitted power in addition to the interference from other nodes (sensors) in adjacent hubs represented by the cross-correlation among Gold/Kasami codes from different groups or sets. Those design parameters and their performance analysis are discussed in Section 3.4 .

\subsubsection{Antenna Development}

The antenna development effort was focused on various antenna structures and geometries optimized for printing on PI sheets in three frequencies of operation- $2.4 \mathrm{GHz}, 900 \mathrm{MHz}$, and $433 \mathrm{MHz}$. The group of candidate antenna structures for use in short-range radio devices included the printed dipole, the microstrip patch, and variants of monopoles. Of particular interest were antenna designs that are not dependent on the separation from the ground plane. Inverted-F antennas (IFAs), shown in Figure 30, with co-planar ground planes and antennae, were chosen as the best design formulation for printing over 1020 mil PI sheets. IFAs are vertically polarized with a donut-shape pattern aligned with the axis coinciding with the vertical axis. Figure 31 shows the dielectric properties of the PI sheet used for printing.

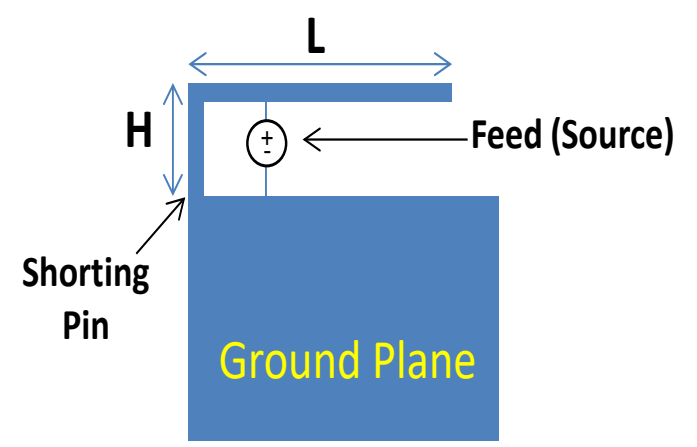

Figure 30 The structure of an inverted-F antenna. 


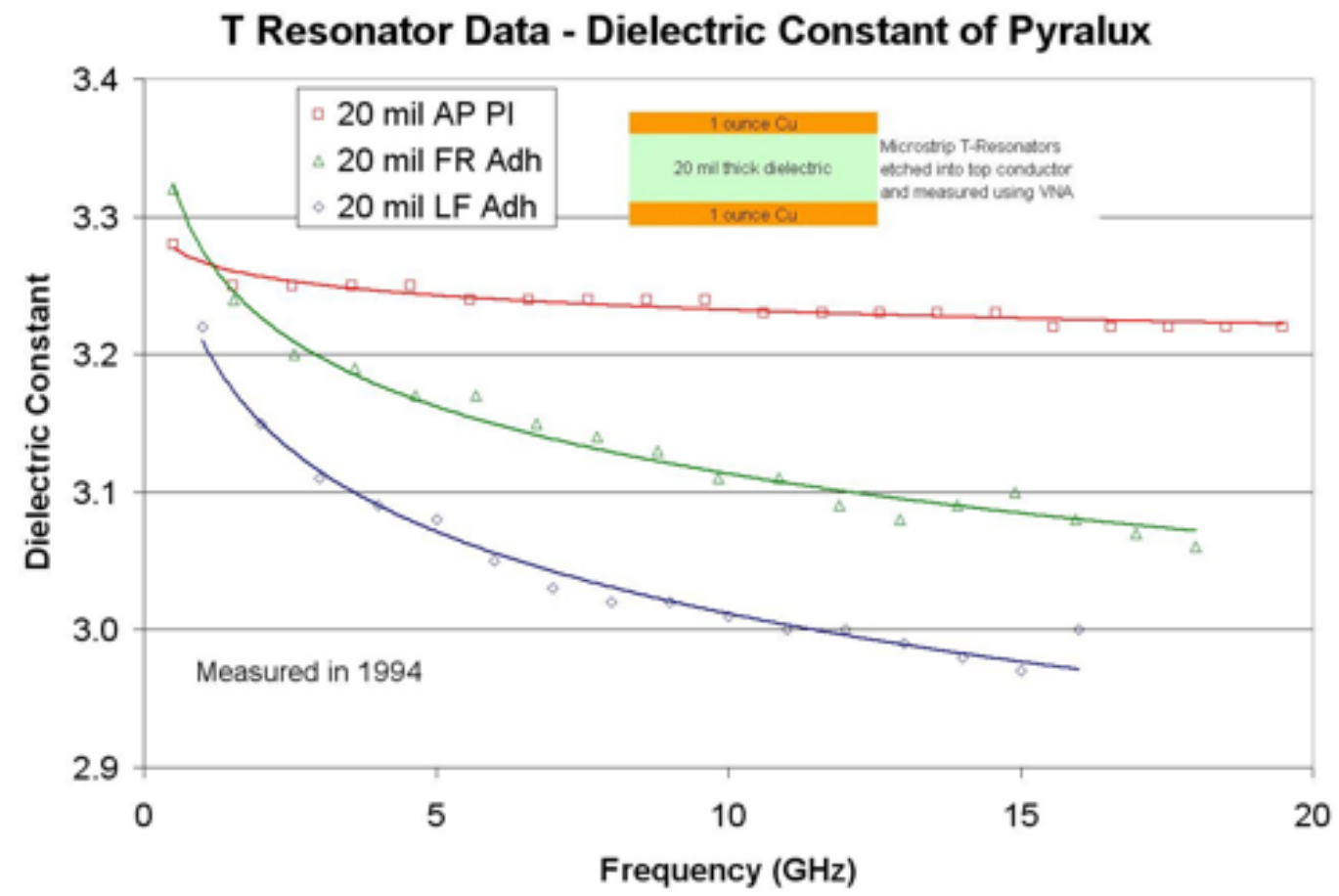

Figure 31 Frequency-dependent dielectric properties of polyimide substrate.

A printed monopole antenna addresses the demands for small size, ease of fabrication and tunability, and low cost for short-range applications. A printed monopole antenna and its high-frequency performance in the $2-3 \mathrm{GHz}$ frequency range are shown in Figure 32. Figure 33 shows the printed IFA. A return loss below $-10 \mathrm{~dB}$ was easily achieved for the monopole design with resonant frequency tuning in the frequency range of 2.0-5.5 GHz. The combination of tunable antenna design, printed conductor performance, and integration on plastic and paper substrates is showing promise for low-cost wireless sensor platform development.
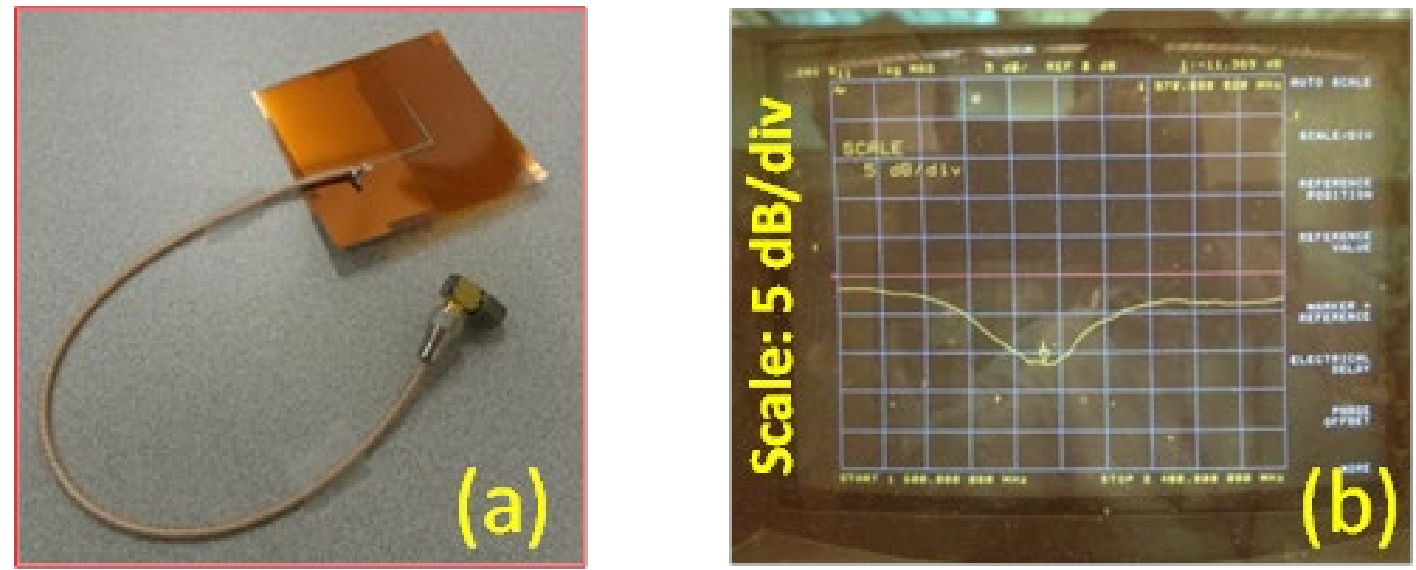

Figure 32 (a) Printed monopole and (b) its characteristics. 


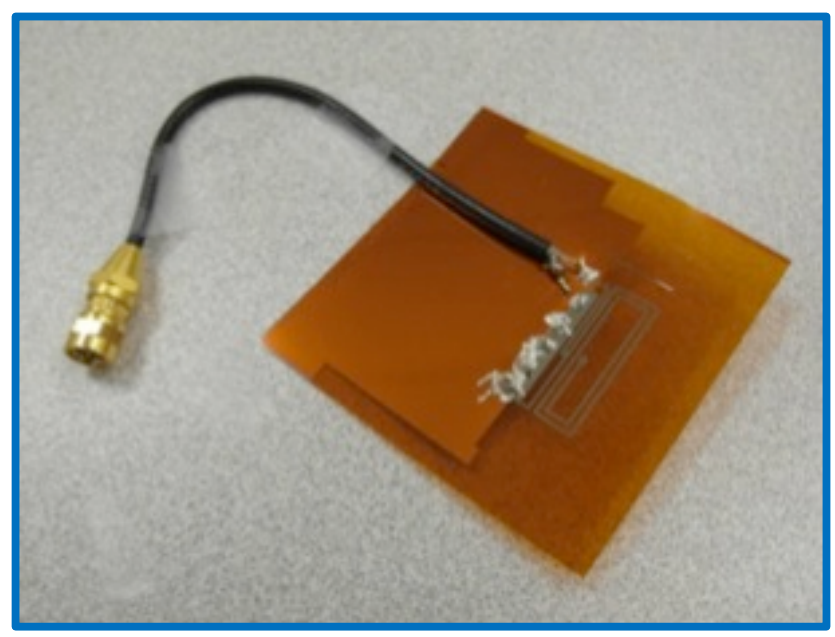

Figure 33 Printed IFA.

The inkjet-printed microwave circuit has been actively investigated by various research groups because of its high potential for building low-cost microwave devices. Various materials can be used for the substrates of this printed microwave circuit. Pyralux AP flexible circuit material had the most interest for us because of its robustness in high-temperature furnaces and its flexibility. Therefore, it can expand the application area of traditional printed microwave circuits to wearable and smart sensor devices.

A $2.4 \mathrm{GHz}$ antenna prototype was built to validate the RF performance of a printed circuit on a Pyralux AP substrate. The slot antenna was used for this design because of its wide bandwidth. Eventually, this design will be optimized to cover a $2.4 \mathrm{GHz}$ and $5.8 \mathrm{GHz}$ dual band. Figure 34 shows the prototype design of a $2.4 \mathrm{GHz}$ antenna with a Pyralux substrate. An SMA (SubMiniature version A) RF connector was attached with conductive epoxy glue. The S-parameter and antenna gain were measured at the University of Tennessee antenna test facility. The S-parameter measurement showed good agreement with the simulation, as shown in Figure 35. The measured antenna gain showed $85 \%$ agreement with the simulated antenna gain. More design optimization of $2.4 \mathrm{GHz}$ for compact size and construction of another prototype of $433 \mathrm{MHz}$ are currently being investigated using these designs, as shown in Figure 36 .

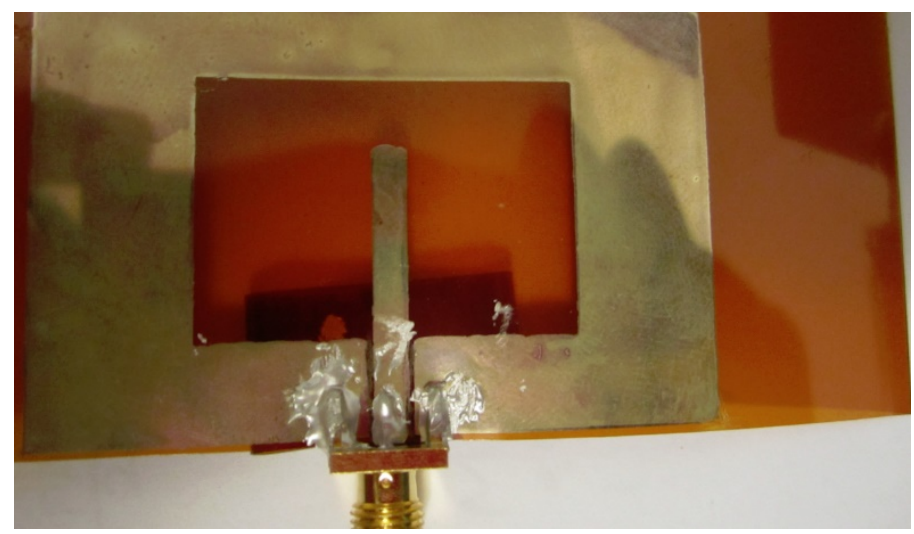

Figure 34 A 2.4 GHz slot antenna with Pyralux substrate. 

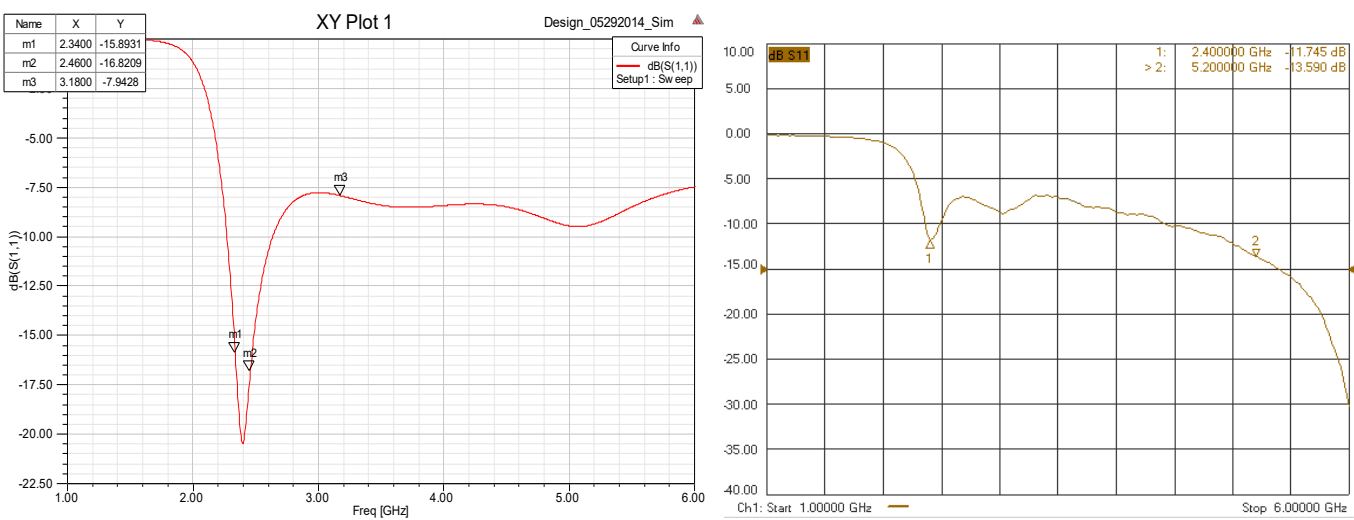

Figure 35 Simulation and measurement results for a $2.4 \mathrm{GHz}$ antenna prototype.

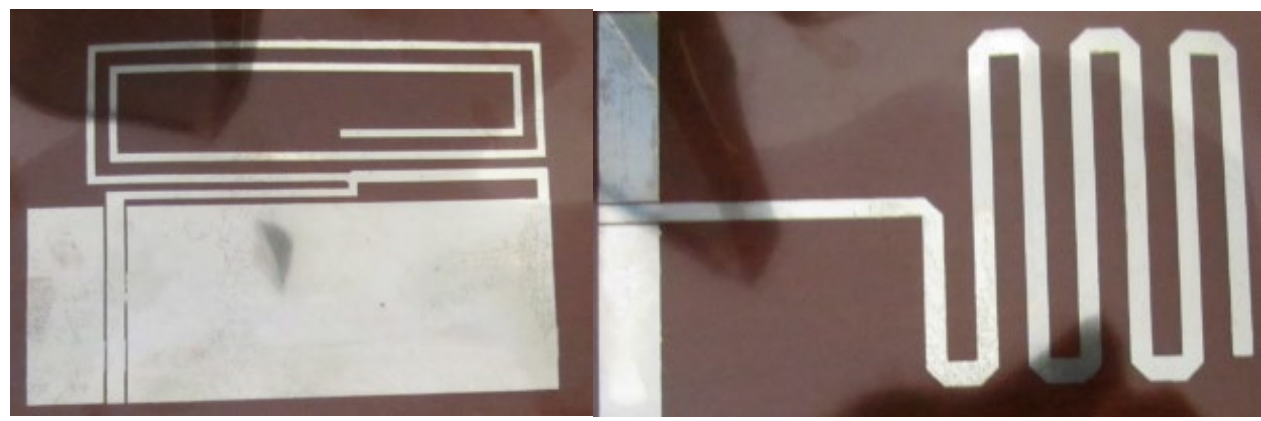

Figure 36 A 433 MHz printed planar IFA and monopole.

\subsubsection{Power Consumption}

The solar cell used in the design is a PowerFilm SP4.2-37. It is specified for outdoor use to produce $4.2 \mathrm{~V}$ at $22 \mathrm{~mA}$, but for typical office indoor use, it produces only $3.5 \mathrm{~V}$ and $350 \mu \mathrm{A}$.

Power can be delivered to the system from the solar cell, supercapacitor, CR2032 battery, or ML2032 battery. When a sensor tag is transmitting a bit, the transmitter chip is supposed to draw $16.8 \mathrm{~mA}$, but this will vary depending on how well the antenna is tuned. And since the bits are on for half the time, the average current draw will be about $9 \mathrm{~mA}$. The following analysis considers the two extreme chip rate cases, those being 2,048 chips/second for 1 second on-the-air time and 32,768 chips/second for 0.062 seconds on-the-air time. It is worth mentioning that the idle current for the sensor tag is significant at about $2 \mu \mathrm{A}$. This is equivalent to one transmission every 1.1 hours at the 2,048 rate or 4.1 minutes at the 32,768 rate.

In this section, the theoretical lifetimes for the various power sources are computed. In all cases, the supercapacitor is still in the circuit because the solar cell will have limited drive, and the recommended load for the CR2032 and ML2032 batteries is a $15 \mathrm{~K} \mathrm{ohm}$ or $0.19 \mathrm{~mA}$ draw. The supercapacitor itself has a $25 \mathrm{ohm}$ internal resistance, but it will cause a drop of only about $0.22 \mathrm{~V}$ during transmission.

For only the solar cell and supercapacitor case, the average current draw must be less than $350 \mu \mathrm{A}$, which equates to a $4.375 \%$ duty cycle. For the 2,048 rate, we could transmit every 23 seconds; and at the 32,768 rate, we could transmit every 1.5 seconds. To determine the running time for the supercapacitor once the lights go out, we computed the available charge in the 0.1 farad supercapacitor when it goes from $3.0 \mathrm{~V}$ to the rated lower voltage of the computer and transmitter, which is $2.0 \mathrm{~V}$. 


$$
\begin{gathered}
q_{3}=C \times V=0.1 \times 3.0=0.3 \text { coulombs } . \\
q_{2}=0.1 \times 2.0=0.2 \text { coulombs } \\
q=q_{3}-q_{2}=0.1 \text { coulombs }
\end{gathered}
$$

A transmission at the 2048 rate requires $1.0 \times 0.008=0.008$ coulombs, so we should be able to transmit 12 times from just the supercapacitor. For the 32,768 rate, we should be able to transmit 192 times. As a result of the idle current, this charge will be consumed in 14 hours. Note also that the solar cell usually charges up the supercapacitor in about 10 minutes.

The CR2032 battery has $240 \mathrm{~mA}$-hours of capacity. Initially, consider the situation where there is no solar cell. In that case, the idle current would deplete the battery in 13 years. At the 2048 chip rate, there could be 100,000 transmissions, and the battery would last for 2.8 years transmitting 4 times per hour. At the 32,768 rate, there could be 1,600,000 transmissions, and the battery would last 90 years. The idle current would obviously be the bigger limitation. The ML2032 battery has $65 \mathrm{~mA}$-hours of capacity. Scaling down the numbers from the CR2032 case, the idle current limits us to 3.5 years. For a 4 times-per-hour transmission rate, the 2,048 rate would deplete the battery in 9 months and the 32,768 rate would deplete it in 24 years. Also note that the recharge time from the solar cell would be $65 / 0.35$ or 185 hours ( 8 days) even if the tag were turned off.

\subsection{PERFORMANCE ANALYSIS}

The correlation properties of Gold/Kasami codes play a major role in the receiver design and performance of the developed CPSK system because they determine not only the level of multiple access interference - i.e., the interference arising from other users of the channel and self-interference due to multipath propagation - but also the code acquisition properties. The interference arising from other users is affected by the cross-correlation properties among different codes of the Gold/Kasami code family; whereas the self-interference due to multipath fading is affected by the auto-correlation properties, that is, the correlation between time-shifted versions of the same code. The code acquisition process is affected by both the cross-correlation and auto-correlation properties.

We initially investigated the correlation properties of the employed 1023-length Gold code set and found out that the cross-correlation function of any pair of sequences has three-valued possibilities $\{-1,63$, $-65\}$. The off-peak auto-correlation function is also three-valued and takes values from the same set. Next, we conducted end-to-end system-level simulation-based studies to investigate the performance of the developed Gold/Kasami CPSK scheme. Figure 37 illustrates the single-user bit-error rate (BER) performance under an additive white Gaussian noise (AWGN) environment and compared it with the conventional BPSK modulation scheme. It can be observed that an effective processing gain of approximately $24 \mathrm{~dB}$ was attained using the proposed approach. Figure 38 illustrates the single-user BER performance under a multipath Rayleigh fading-type environment compared with the conventional DBPSK modulation scheme. The same observation holds as in the AWGN channel case. 


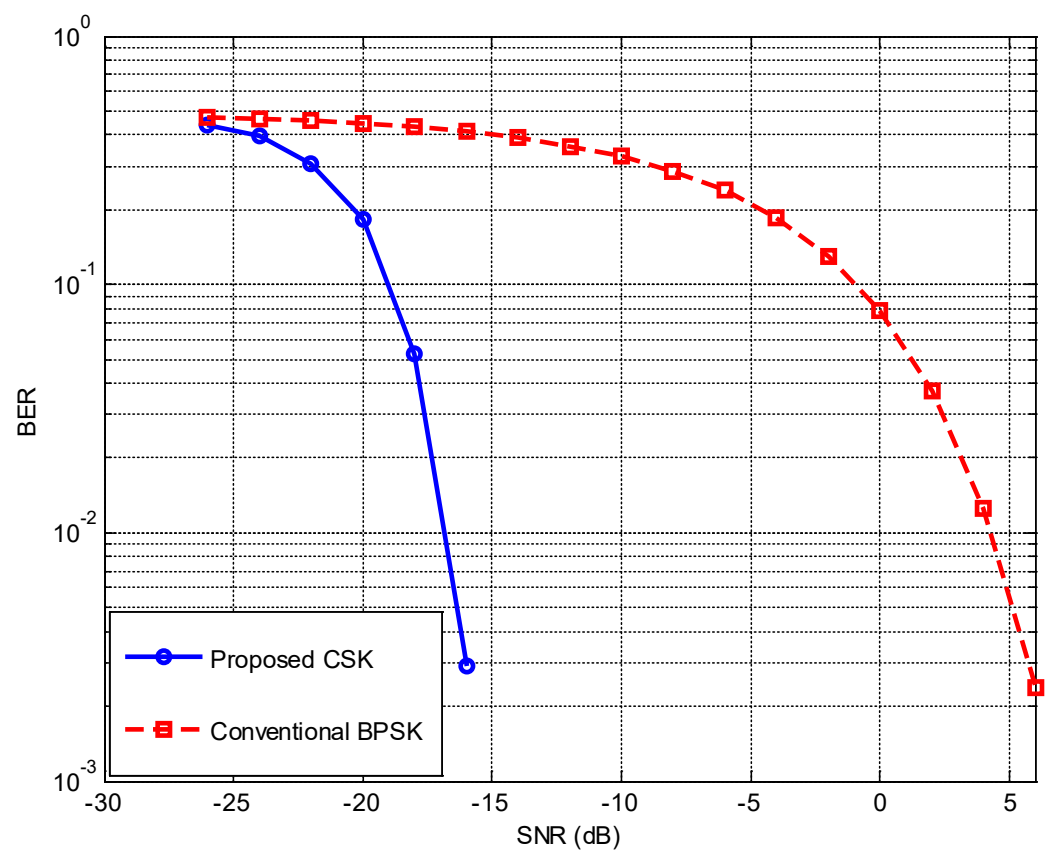

Figure 37 Single-user BER performance for the CPSK signaling scheme under an AWGN environment.

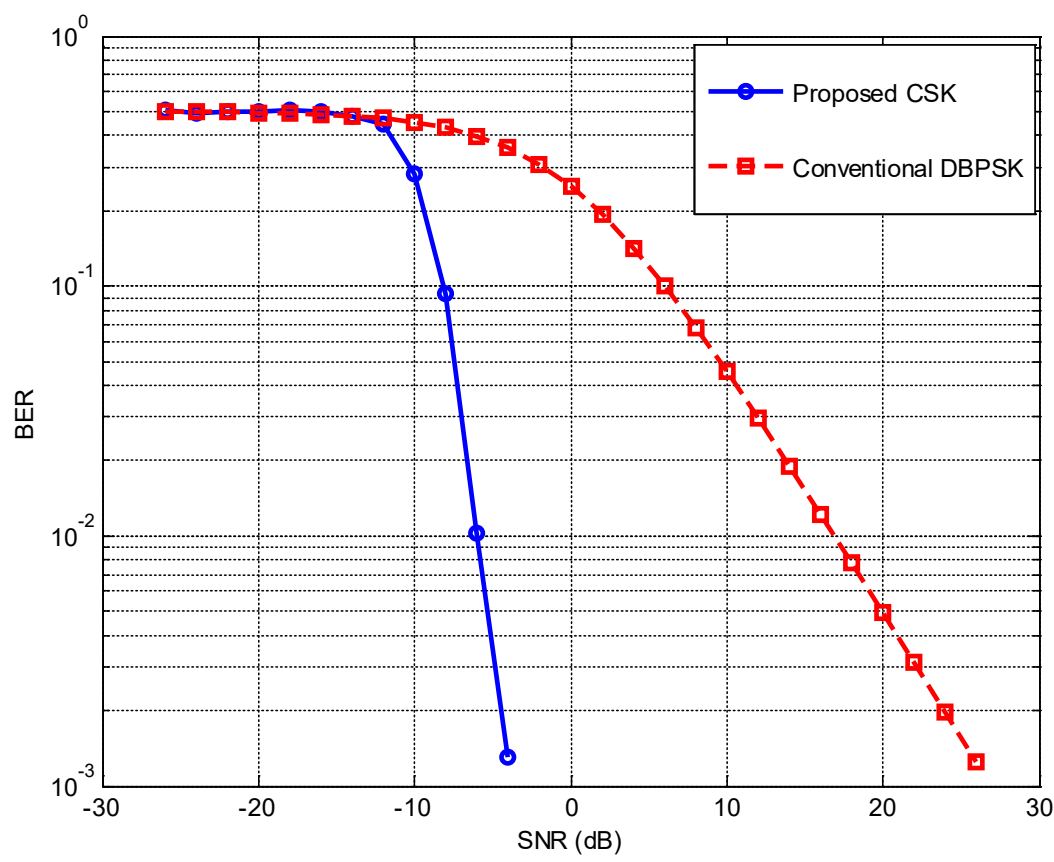

Figure 38 Single-user BER performance for the CPSK signaling scheme under Rayleigh fading-type environment.

Next, we wanted to investigate the multiple access capability of the developed CPSK scheme, which is based on asynchronous TDMA. This scheme can be implemented as random access, where channel assignment is performed in a distributed, random fashion. In this case, a collision may happen if two or more transmitters transmit packets at the same time. To compute the success rate for such an approach, let the number of users in the network be $N$, let all users transmit packets with a fixed (average) length of $T$ 
seconds, and let each user transmit with a fixed probability $p$ in the time period $T$. A packet will be in a collision if and only if another transmission begins in the vulnerable "danger" period, which starts $T$ seconds before a sensor initiates its transmission and ends $T$ seconds after the sensor completes its packet. So, for a transmission to be successful, no other sensor should transmit during the time period of $2 T$. During this time period, the average number of packets transmitted will be $2 \mathrm{~Np}$. If we assume that the traffic model follows a Poisson process, which is a realistic assumption [23], then the probability of a successful transmission is described by

$$
\operatorname{Pr}(\text { Successful Transmission })=\exp (-2 N p)
$$

where $\exp (\cdot)$ is the exponential function. Figure 39 and Figure 40 demonstrate the success rates of this multiple access scheme under packet transmission times of one second and 0.1 second, respectively.

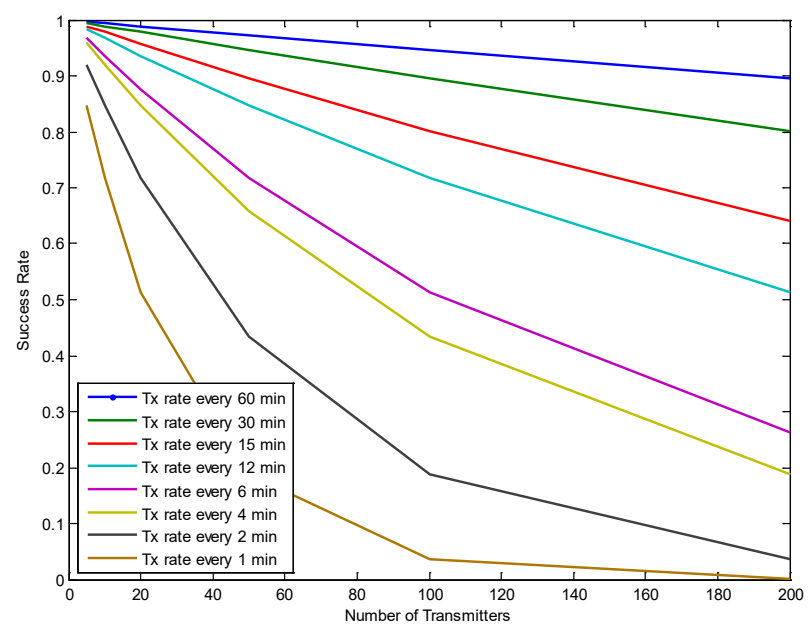

Figure 39 Success rates of the original multiple access scheme at a packet transmission time of 1 second.

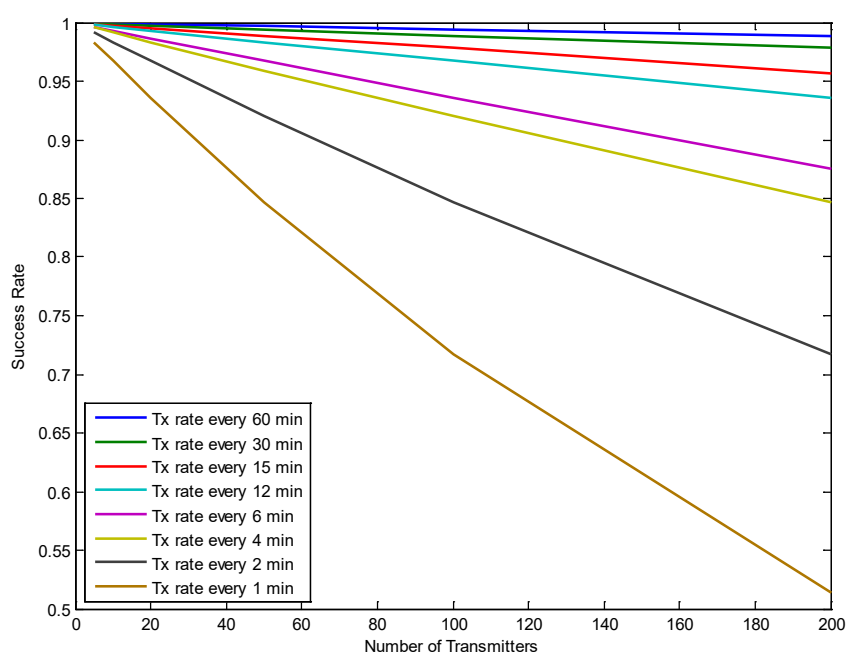

Figure 40 Success rates of the original multiple access scheme at a packet transmission time of 0.1 second.

If we consider the acceptable success rate to be 0.95 and at a packet transmission time of 1 second, we observe (from Figure 39) that 100 nodes per hub can be deployed only if the transmission rate for each node is no faster than once every 60 minutes. On the other hand, at a packet transmission time of 0.1 
second, we observe (from Figure 40) that 100 nodes per hub can be deployed only if the transmission rate for each node is no faster than once every 12 minutes.

Now, assume that the performance of the building energy efficiency mechanism will not be greatly affected if a few sensors miss only one transmission packet in sequence. We believe that the energy efficiency mechanism can tolerate this modified packet loss scheme. In this case, "failure" is defined as missing two or more consecutive transmissions; and "success" is defined as successfully receiving every other transmission packet. No closed-form expression exists for computing the probability of success for this case, so it is computed using Monte Carlo simulations. Figures 39 and 40 demonstrate the success rates of the modified packet loss scheme under packet transmission times of one second and 0.1 second, respectively. Again, if we consider an acceptable success rate to be 0.95 and at a packet transmission time of 1 second, we observe (from Figure 41) that 100 nodes per hub can be deployed only if the transmission rate for each node is no faster than once every 15 minutes. On the other hand, at a packet transmission time of 0.1 second, we observe (from Figure 42) that 100 nodes per hub can be deployed only if the transmission rate for each node is no faster than once every 2 minutes. Moreover, when there are fewer transmitters per hub, higher transmission rates can be achieved.

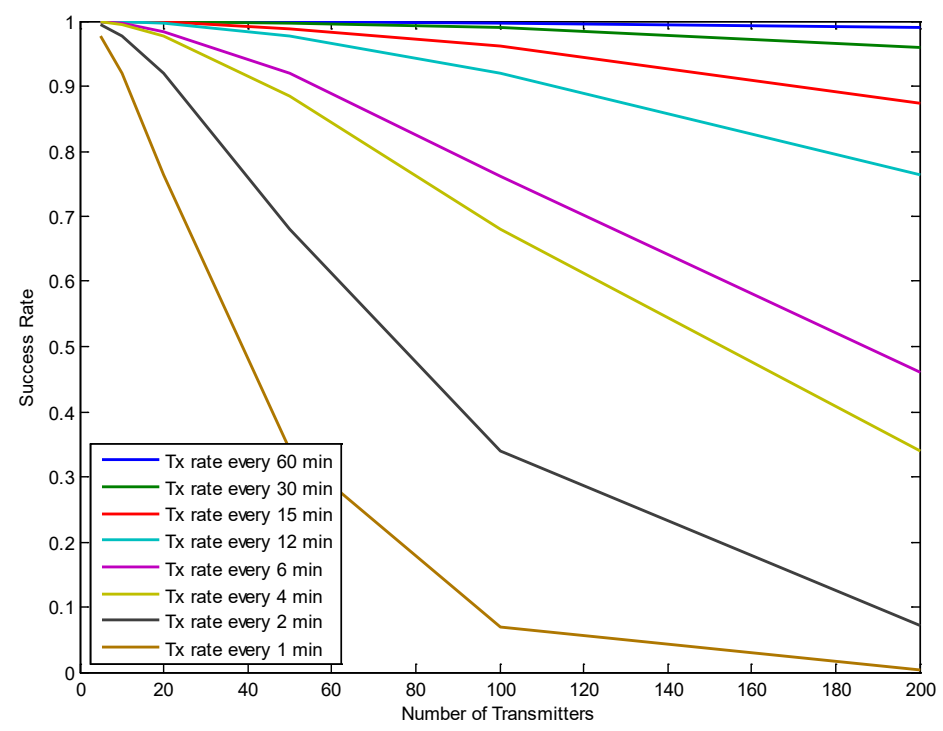

Figure 41 Success rates of the modified multiple access scheme at a packet transmission time of 1 second. 


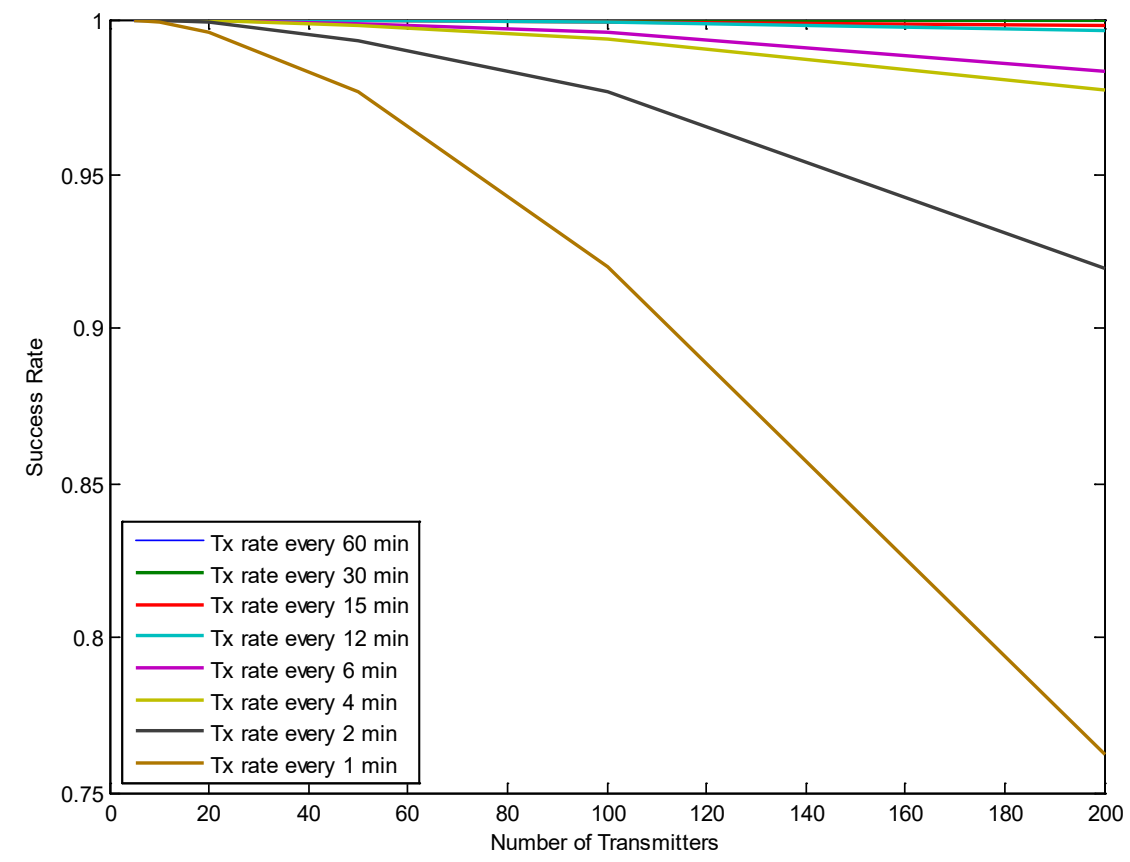

Figure 42 Success rates of the modified multiple access scheme under packet transmission time of 0.1 second.

Now we consider the interference among transmitters across different hubs. It depends on the crosscorrelation among shifted Gold/Kasami codes from different sets, since different LFSR tap combinations are employed among different hubs. This aspect has an impact on the size and coverage area of each hub. Figure 43 and Figure 44 demonstrate the BER performance due to interference resulting from transmitters at different hubs under AWGN and Rayleigh fading-type environments, respectively. The worst-case scenario is considered in the analysis by assuming equal received powers from the intended transmitter and the interference transmitters from other hubs (assuming the near-far problem always exists). The high interference rejection capability of such an approach can be seen under the different environments, especially the multipath fading that represents a highly dense indoor environment, such as a typical office building. This fading is because cross-correlations among different Gold/Kasami codes from different tap combinations are still low (processing gain is about $18 \mathrm{~dB}$ ), but not as good as cross-correlations among different Gold/Kasami codes from the same set (processing gain is about $24 \mathrm{~dB}$ ). Improved performance is anticipated in real scenarios since the near-far problem does not always (but often) exists. 


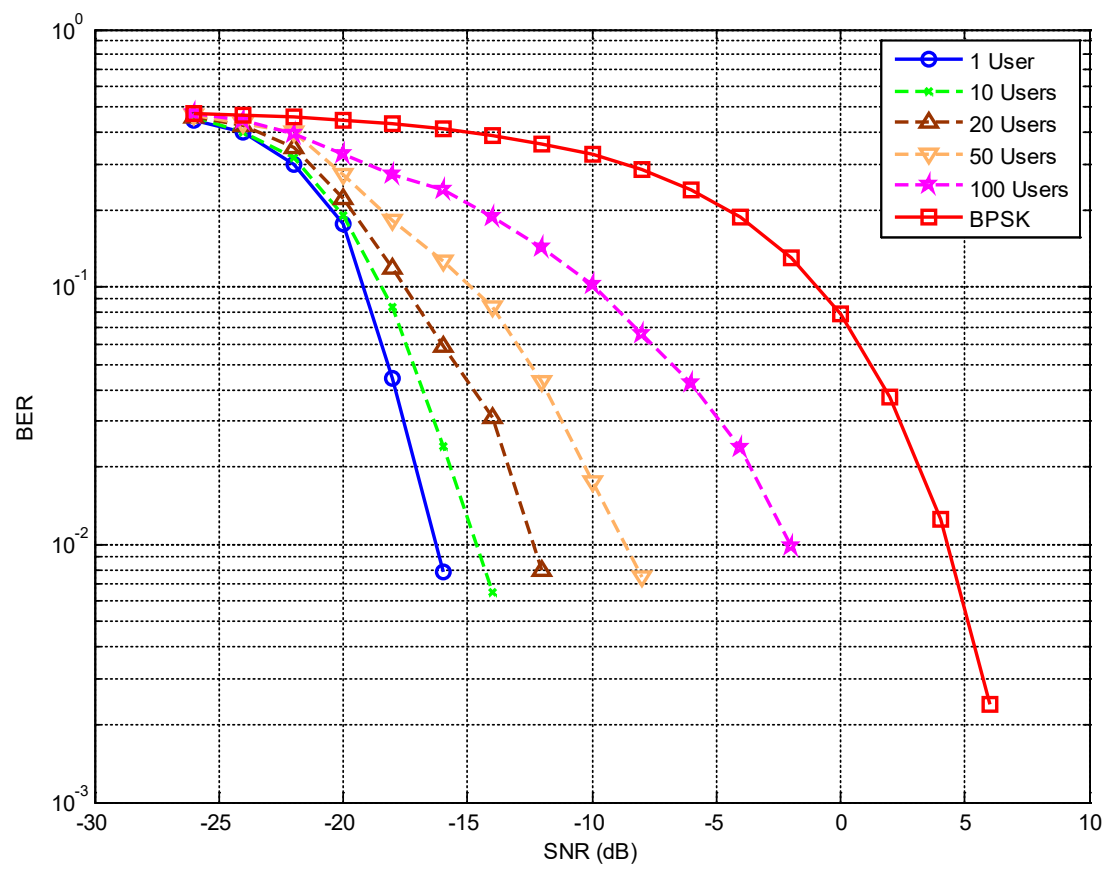

Figure 43 BER performance of the developed CPSK signaling scheme

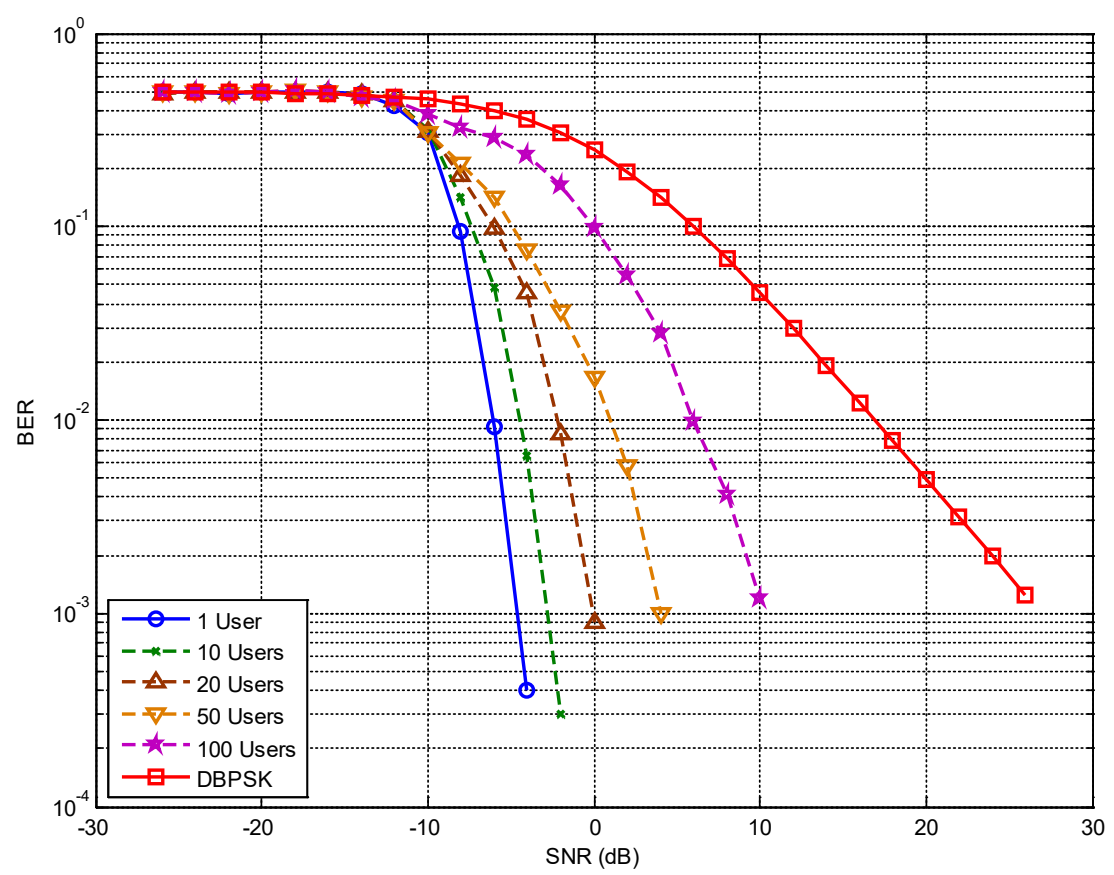

Figure 44 BER performance of the developed CPSK signaling scheme due to interference resulting from transmitters at different hubs under a Rayleigh fading-type environment.

Next, we analyze the additional consumption of spectrum to implement CPSK. The encoded data frame delivers 26 bits of data but transmits 1023 bits on the channel. For our purposes, this factor-of-40 consumption increase is offset by the smaller power requirements of the transmitter. Achieving the full process gain of $30 \mathrm{~dB}$, or a power factor of 1000 , would reduce the overall energy requirement by a factor 
of 10. At this time, we have not obtained the full $30 \mathrm{~dB}$ process gain, yet the $23 \mathrm{~dB}$ gain allows us to reduce the cost of the transmitter and use energy harvesting. Although this is very beneficial for our objective, we still need to address whether we are wasting the public's spectrum resources. If we use the interference rejection properties of spread spectrum, neighboring systems could be closer together, resulting in an equivalent consumption of spectrum compared with a conventional system within a given geographical area. To describe the spectrum consumption, we introduce the concept of bandwidth*area. For a conventional system, there must be a buffer area between a user and a neighboring user on the same frequency, as illustrated in Figure 45. Nine simultaneous users for this scenario would require nine separate frequencies, as shown in Figure 46. For the spread-spectrum system, if spread spectrum allows nine users on the same frequency, the radio could use nine times as much bandwidth for the same overall spectrum usage. Thus, the area*bandwidth would be the same for both cases.

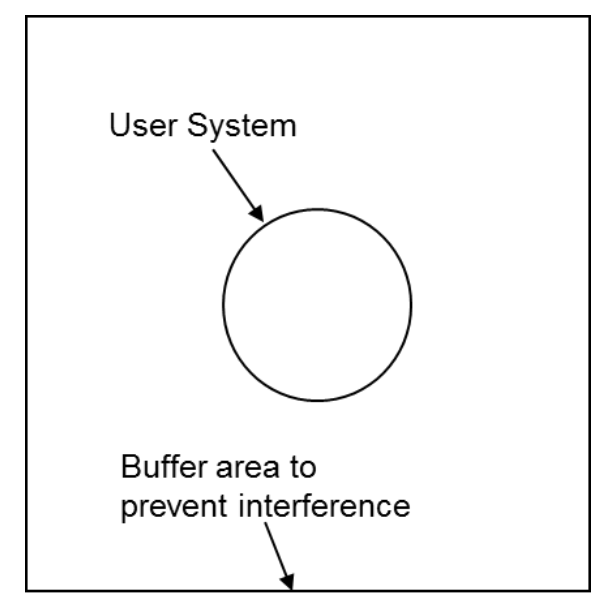

Figure 45 Frequency reuse buffer area.

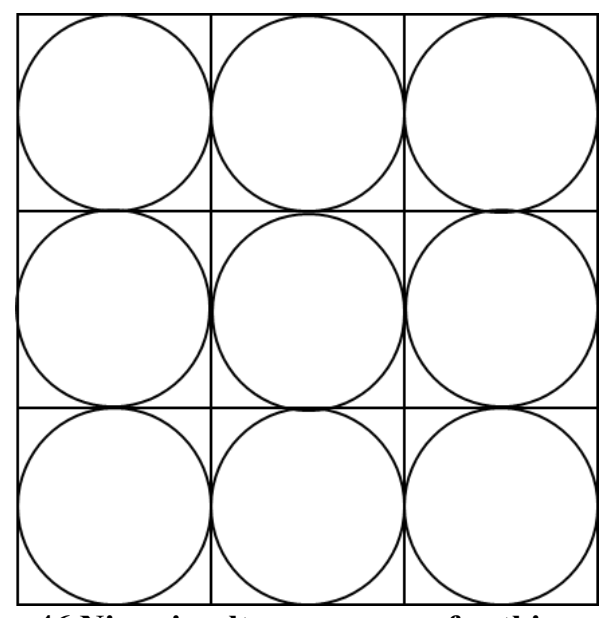

Figure 46 Nine simultaneous users for this scenario would require nine separate frequencies.

\section{Required SNR for $10 \mathrm{E}-6$ error rate is $17 \mathrm{~dB}$ for standard $\mathrm{AM}$}

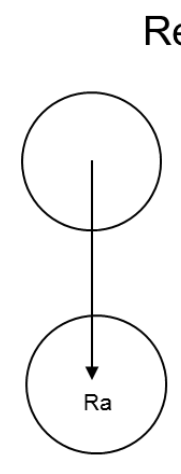

Regular AM

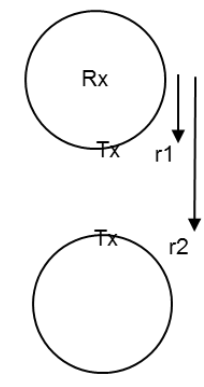

Let $\mathrm{r} 1=1$

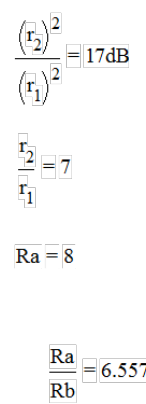

Spread Spectrum $30 \mathrm{~dB}$ Process Gain

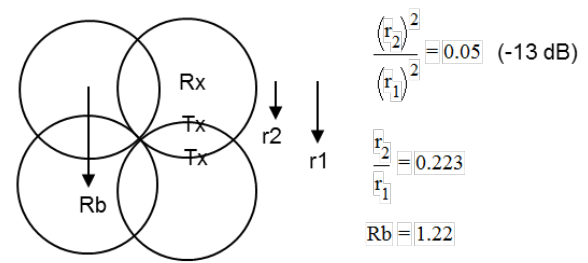

Figure 47 An example showing that frequency reuse in spread-spectrum systems would recover most of the extra bandwidth compared with conventional AM.

\subsection{EXPERIMENTAL RESULTS}

In addition to performance verification via simulations, several practical experiments were conducted to evaluate the performance of the implemented CPSK prototype. The key experiments measured the 
receiver sensitivity, throughput (baud rate), bandwidth, battery life, data decode times, and real testing in a typical office building.

Receiver sensitivity is measured by using a Rohde and Schwarz SME 03 signal generator. It can be modulated using AM or FM signals by feeding the baseband signal from one of the tags. The available tag chip rates are 2048, 4096, 8192, 16384, and 32768 chips per second. We used 100\% modulation AM depth, or on-off-keying (OOK). Note that the power level stated by the signal generator represents the power at the $50 \%$ midpoint. For OOK, the peak is twice the voltage, or $6 \mathrm{~dB}$ higher, so the true average power is $3 \mathrm{~dB}$ greater that what is indicated. We adjusted the power until about half the packets came through; this power level is called the "sensitivity." There was also an adjustable threshold setting that we typically set at 30, which means we triggered on signals that were a factor of 30 above the average signal level. This approach was like a squelch, and it was set depending on how much false triggering we could accept. We typically used the value 30 when a real antenna was connected to the receiver because it was the level needed to reject available real-world traffic $f$ we were connected only to a signal generator for sensitivity tests, we set the threshold to 18 . The FM FSK version was based on $2.5 \mathrm{KHz}$ deviation and a chip rate of 4096 symbols per second, which was very similar to the P25 standard. As an experiment, we also demonstrated the receiver at 2048 chips per second.

We had baseline receivers that have four-pole IF filter bandwidths of 7.5, 15, and $30 \mathrm{KHz}$. Ideally, we should have had a $3.75 \mathrm{KHz}$ filter for our 2048 chip rate, but such a filter was not available. So, we used a digital signal processor-based system running on an FPGA that took a $300 \mathrm{KHz}$ IF signal, downconverted it to a baseband signal, and filtered that signal with an ideal raised-cosine finite impulse response (FIR) filter. We had a version of the FIR filter for the 2048 rate and for the 4096 rate. This FIR system should be the best possible, but the baseline system already worked almost as well. For FM use, there was another version of the $300 \mathrm{KHz}$ FIR system that implemented a frequency discriminator. And finally, we also had an op-amp version of a low-pass-filter at $2 \mathrm{KHz}$ that had four poles.

Initially, we considered the AM with a 1023 length spread-spectrum code. The transmission consisted of 1023 chips for the preamble and 1023 chips for the payload. The first baud rate considered was the 2048 chip rate, because it was the slowest rate and would provide the best sensitivity. The higher chip rates should scale to it proportionally; for example, a chip rate twice as fast should have double the noise bandwidth, so the sensitivity should be reduced by $3 \mathrm{~dB}$. There were several subtle variations of the receiver configuration, and the results for these various configurations and chip rates are presented in Table 4.

Table 4 Experimental receiver sensitivity testing for the CPSK prototype using AM signal with a 1023 length spread-spectrum code.

\begin{tabular}{|l|c|c|}
\hline \multicolumn{1}{|c|}{ Configurations } & \multicolumn{2}{c|}{ Receiver sensitivity (dBm) } \\
\hline \multicolumn{1}{|c|}{ Chip rate 2048 } & Nominal & Actual \\
\hline Baseline 7.5 BW, threshold 18 & -146 & -143 \\
\hline Baseline 7.5 BW, threshold 30 & -143 & -140 \\
\hline Baseline 7.5 BW, FIR 2 K filter, threshold 18 & -148 & -145 \\
\hline Baseline 7.5 BW, FIR 2 K filter, threshold 18, ZX60-P33ULN & -149 & -146 \\
\hline Baseline 7.5 BW, FIR 2 K filter, threshold 30 & -145 & -142 \\
\hline Baseline 7.5 BW, FIR 4 K filter, threshold 18 & -147 & -144 \\
\hline Baseline 7.5 BW, FIR 4 K filter, threshold 30 & -144 & -141 \\
\hline Chip rate 4096 & Nominal & Actual \\
\hline
\end{tabular}




\begin{tabular}{|l|c|c|}
\hline Baseline 7.5 BW, threshold 18 & -144 & -141 \\
\hline Baseline 7.5 BW, threshold 30 & -142 & -139 \\
\hline Baseline 7.5 BW, FIR 4 K filter, threshold 18 & -145 & -142 \\
\hline Baseline 7.5 BW, FIR 4 K filter, threshold 30 & -143 & -140 \\
\hline \multicolumn{1}{|c|}{ Chip rate 4096 } & Nominal & Actual \\
\hline Baseline 30 BW, threshold 18 & -138 & -135 \\
\hline
\end{tabular}

For the frequency shift keying (FSK) tests, we used a $2.5 \mathrm{KHz}$ deviation and demonstrated both 2048 and 4096 chip rates. We also had two different versions of the discriminator that were bandwidth optimized for the 2048 and 4096 rates. We also tried using some standard FM receivers (walkie-talkies) and fed their audio (baseband) into the FPGA decoder. The results are illustrated in Table 5.

Table 5 Experimental receiver sensitivity testing for the CPSK prototype using FSK modulation signal.

\begin{tabular}{|l|c|}
\hline \multicolumn{1}{|c|}{ Configurations } & Receiver sensitivity (dBm) \\
\hline \multicolumn{1}{|c|}{ Chip rate 2048 } & -142 \\
\hline Baseline 7.5 BW, FIR 2 K filter, threshold 18 & -140 \\
\hline Baseline 7.5 BW, FIR 2 K filter, threshold 30 & -141.5 \\
\hline Baseline 7.5 BW, FIR 4 K filter, threshold 18 & -140 \\
\hline Baseline 7.5 BW, FIR 4 K filter, threshold 30 & -138 \\
\hline $\begin{array}{l}\text { Radio Shack scanner with MiniCircuits ZX60-P33ULN preamp } \\
\text { and 4.5 KHz deviation, threshold 18 }\end{array}$ & \\
\hline \multicolumn{1}{|c|}{ Chip rate 4096 } & -141 \\
\hline Baseline 7.5 BW, FIR 4 K filter, threshold 18 & -139 \\
\hline Baseline 7.5 BW, FIR 4 K filter, threshold 30 & \\
\hline
\end{tabular}

It is typical for a radio to miss the theoretical value by few decibels. Theoretically, the code length of 1023 should provide $30 \mathrm{~dB}$ of processing gain. Table 6 illustrates the theoretical receiver sensitivity calculations.

Table 6 The theoretical receiver sensitivity of the CPSK signaling scheme.

\begin{tabular}{|lc|}
\hline kTb noise (dBm/Hz) & $-\mathbf{1 7 4}$ \\
\hline 2 Hz bandwidth & +3 \\
\hline 4 times extra Rx BW & +6 \\
\hline Receiver noise figure & +3 \\
\hline Needed SNR & +17 \\
\hline Total & $-\mathbf{1 4 5}$ \\
\hline
\end{tabular}

Further investigation was conducted to determine the reasons for missing the theoretical receiver sensitivity by a few decibels. A search of the literature showed that one fundamental limitation of AM sensitivity was evoked. There is a fundamental limitation on how much a signal can degrade before process gain can be used to reconstruct the signal. If the signal is already unintelligible because of the AM detection, then no amount of process gain will reconstruct the signal. 
The key limitation is the randomness of the phase relationship between the carrier of the desired signal and the background noise. Figure 48 shows the total envelope voltage $V t$, which is the vector sum of the signal voltage $V s$ and the noise voltage $V n$. There will be a net gain in the total voltage if $V n$ and $V s$ combine constructively, or a net loss if they combine destructively. The angle $S$ represents the probability that the net gain will be positive. When the value of $S$ approaches $50 \%$ there will be no net gain. This limitation is independent of the amount of processing gain from the spread-spectrum system; therefore, this limitation cannot be improved by adding more processing gain.

Figure 49 illustrates the angle $S$ versus the SNR $V s / V n$ in decibels. It is observed that the -10 to $-20 \mathrm{~dB}$ SNR range is where the net gain becomes zero and the signal becomes unrecoverable. This agrees with our observation that the signal was not recoverable with a $-18 \mathrm{~dB}$ SNR but instead needed an SNR in the range of $-8 \mathrm{~dB}$. A key point to note is that this limitation could be overcome if the phase of the carrier were known. The system could observe the signal at just that phase and reject the noise from the other phases. GPS is a special case where the satellites send a coarse/acquisition (C/A) signal that does not require as much processing gain to receive. The $\mathrm{C} / \mathrm{A}$ signal provides information about the carrier phase of the P-code, which enables the full processing gain to be used in recovering the P-code signal.

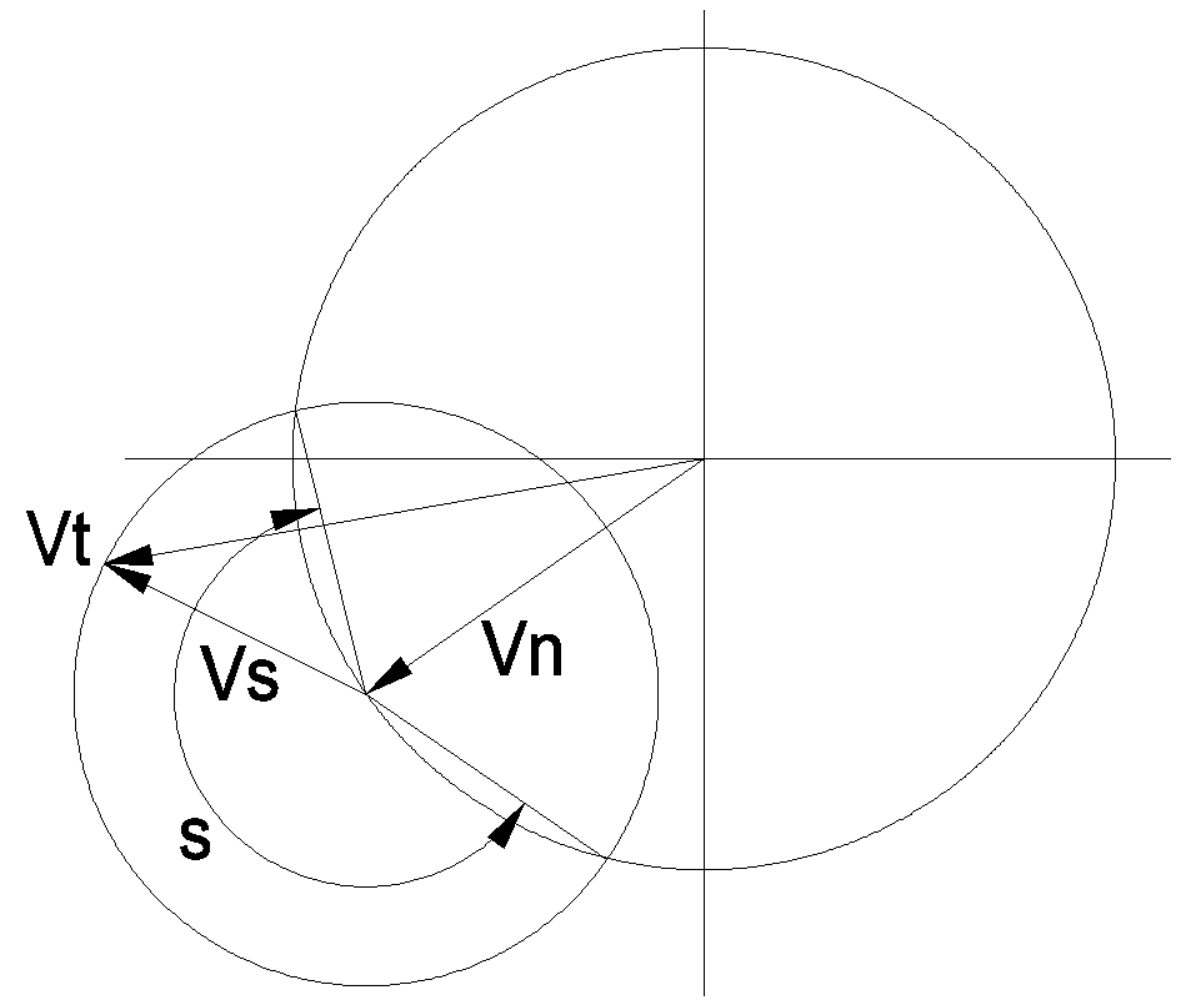

Figure 48 Illustration of the key limitation in the randomness of the phase relationship between the carrier of the desired signal and the background noise. 


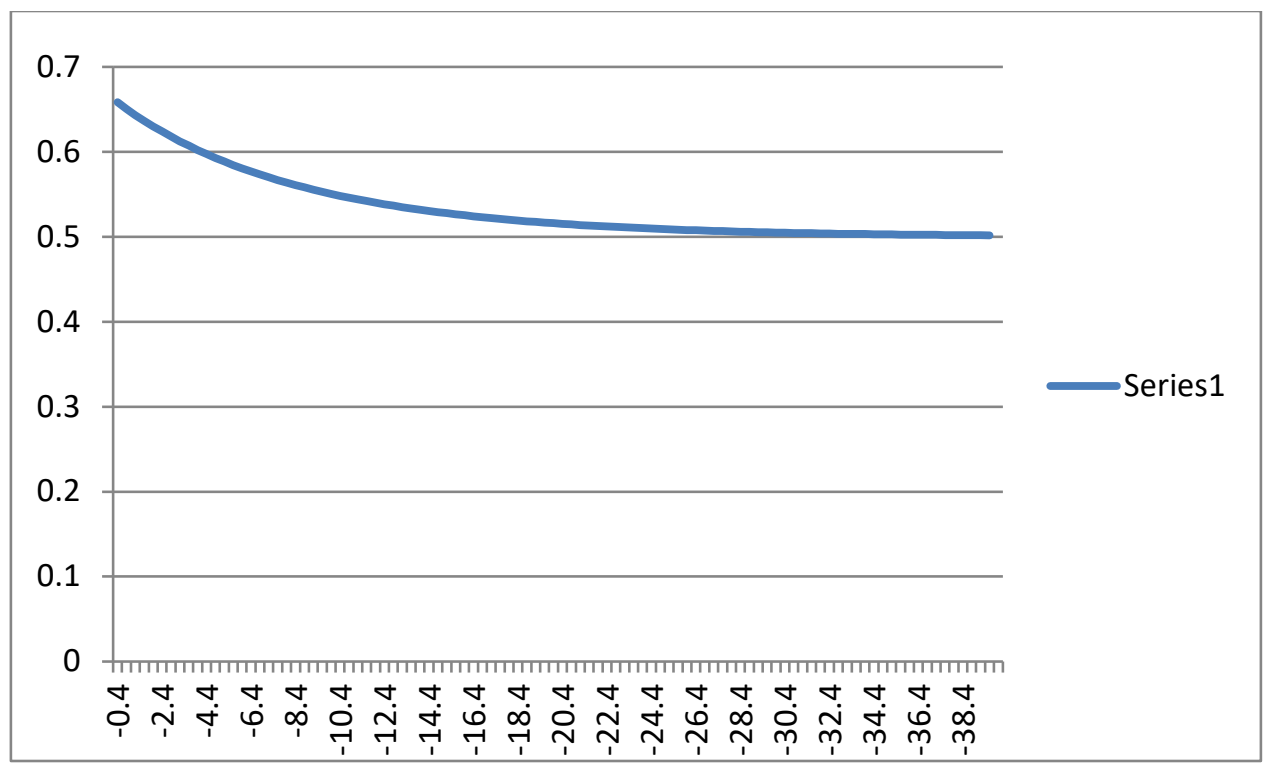

Figure 49 Illustration of the angle $S$ versus the signal-to-noise ratio $\mathrm{Vs} / \mathrm{Vn}$ in decibels.

The second experiment involved conducting coverage range testing of the implemented CPSK transceiver prototype in a typical office building. A highly dense three-story office and laboratory building at ORNL was selected for the experiment. It consists of brick walls for the labs and metal walls for the offices. The CPSK transmitter prototype with an output power of seven $\mathrm{dBm}$ was placed in a fixed location as shown in Figure 50 (represented by the red star). The receiver prototypes were placed at different locations in the building (represented by the green lightning shapes in Figure 50) and measured the packet success rates (represented in numbers) at those locations. It was found that the transmitted packets were correctly detected at the receiver at most locations, and the measured coverage range was approximately 300 feet. The same experiment was repeated on the second floor of the same building (while the transmitter was kept at the same location on the first floor). This scenario is demonstrated in Figure 51. The same observations held as in the first floor experiments, and again the measured coverage range was about 300 feet.

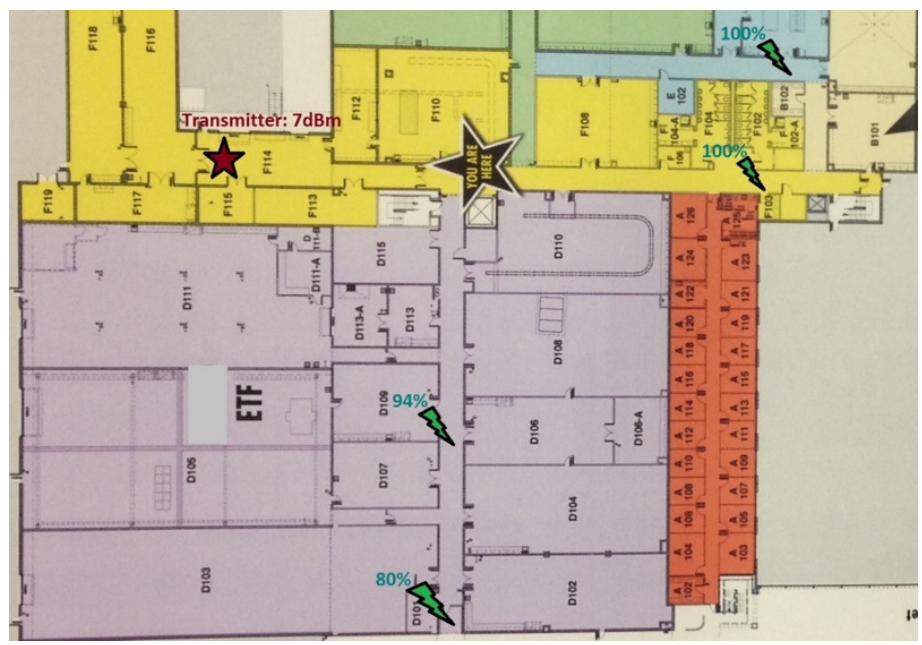

Figure 50 Coverage range testing on the first floor: packet success rates are measured at different locations in a typical office building. 


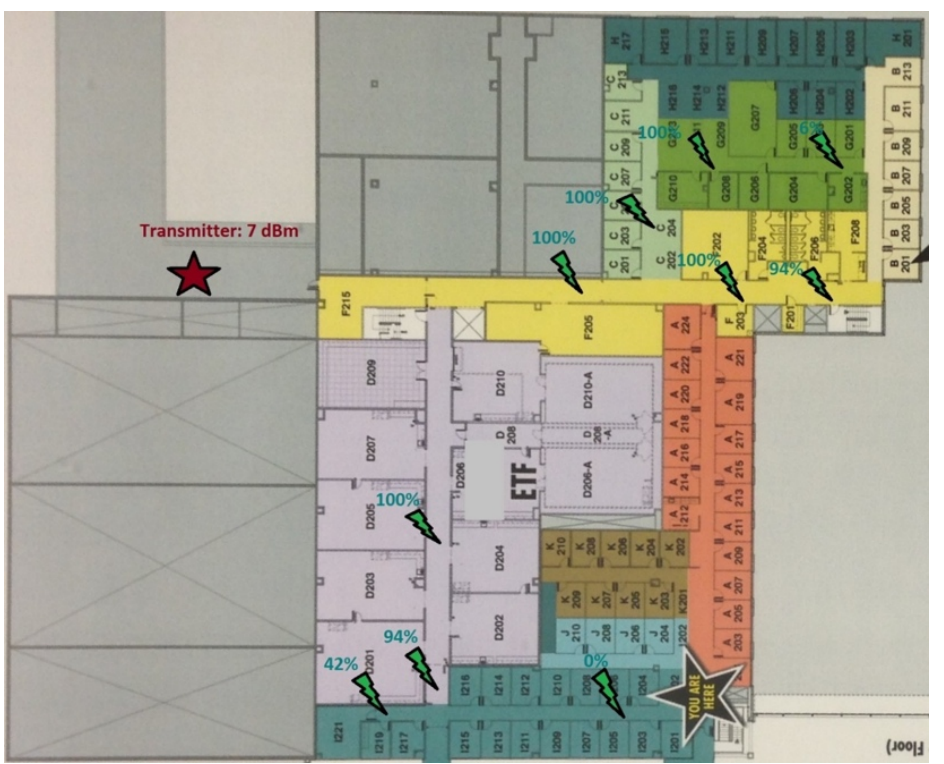

Figure 51 Coverage range testing on the second floor: packet success rates are measured at different locations in a typical office building.

Furthermore, a few free space field tests were conducted for the prototype transmitter. We were able to successfully transmit 3 miles to another point in free space. At another point marginally over the horizon, we were able to sense packets at 10 miles but with bad parities.

\subsection{PROTOTYPE MANUFACTURING}

Molex successfully manufactured prototype versions of the devices (chargeable and rechargeable) and delivered them to ORNL for testing on March 24, 2017. ORNL successfully programmed the devices and tested them for data transmission. All devices met the metrics for circuit functionality, antenna performance, sensor data accuracy, and network functionality. Figure 52 shows multiple CAD views of the prototype. Figure 53 shows the sheet-to-sheet screen printing process. Figure 54 shows the nodes and new receiver prototypes. ORNL is currently conducting extensive mechanical, network, and electrical characterization of nodes. Appendix A describes the testing results for prototype nodes at the ORNL Flexible Research Platforms. 

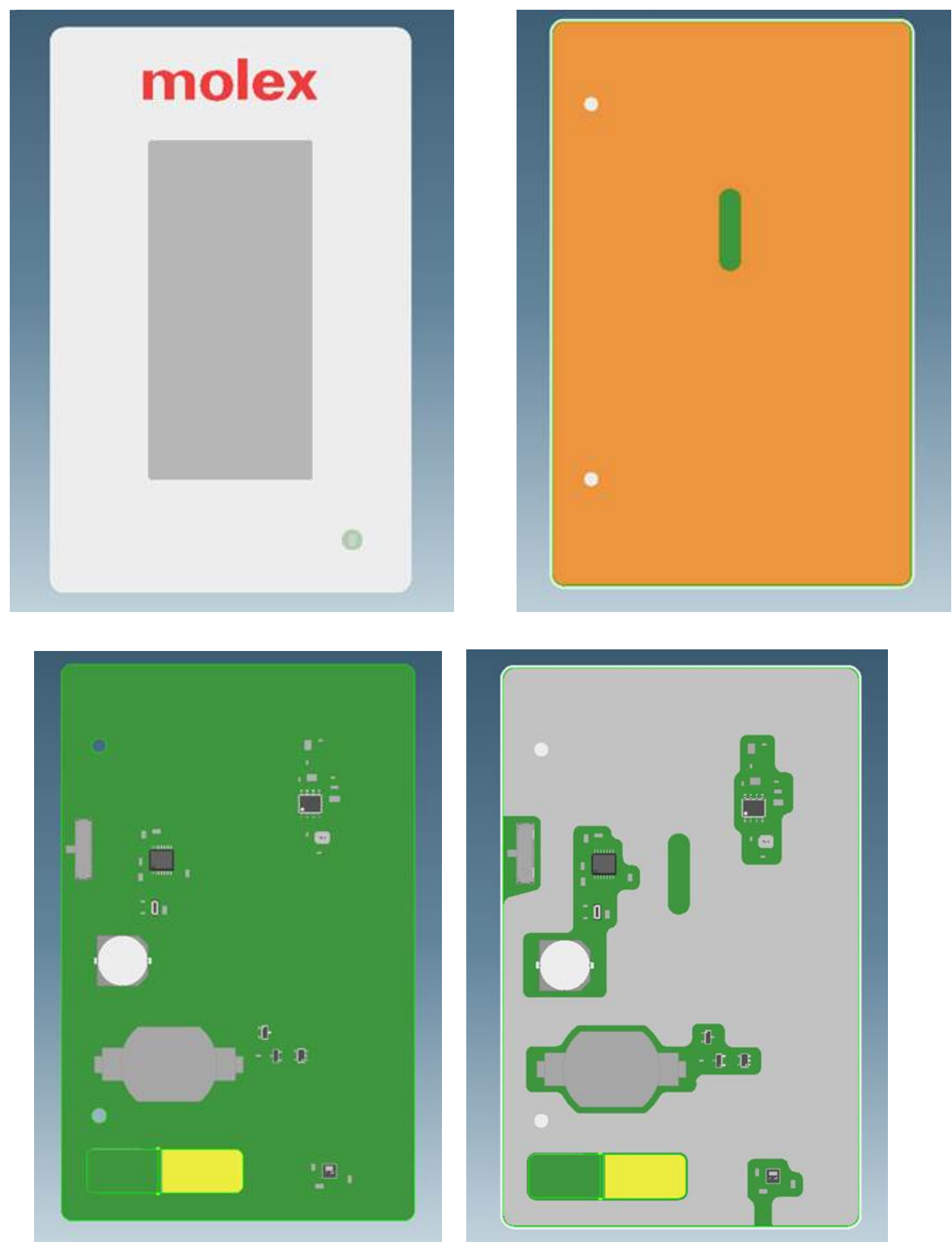

Figure 52 Final CAD views of manufactured prototypes 


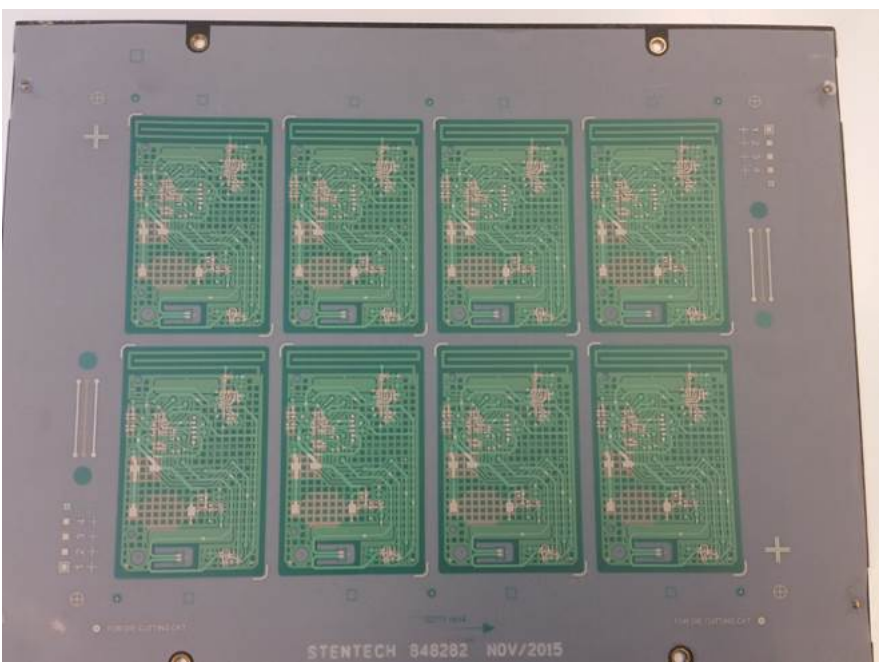

Figure 53 Screen printing in process.
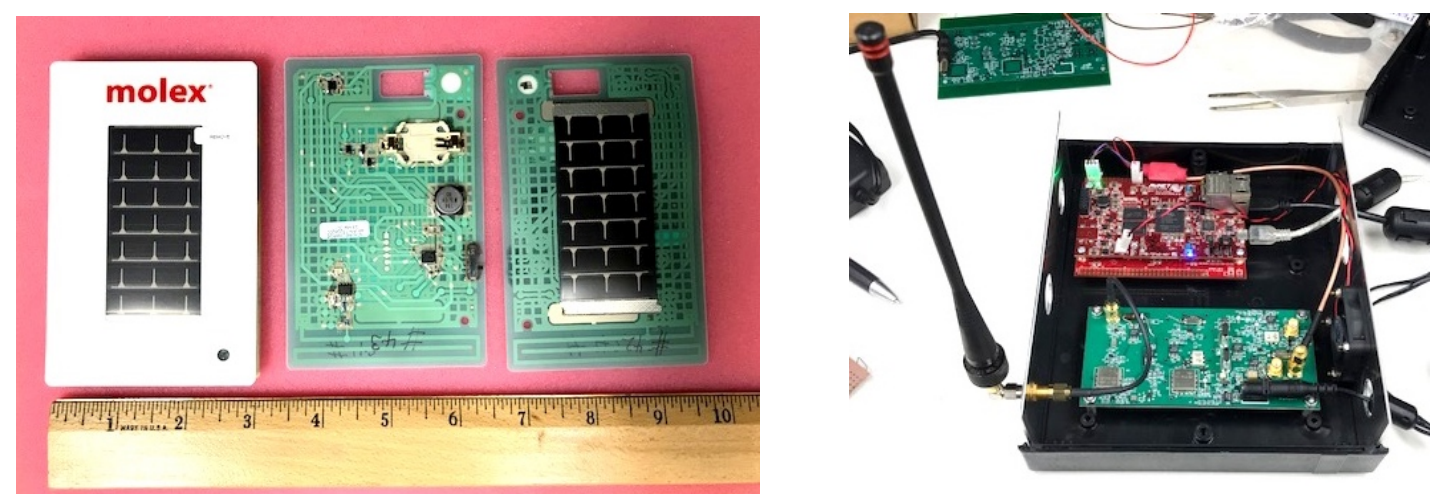

Figure 54 Wireless sensor node prototypes and receiver.

\section{SUBJECT INVENTIONS/PUBLICATIONS}

1. Pooran C. Joshi, Stephen M. Killough, and Phani Teja Kuruganti. "Low-cost printable wireless sensors for buildings applications," US Patent 9729193, August 8, 2017. The patent includes the processing for version 1 printed sensors and the CPSK-based communication scheme.

2. Pooran Joshi, Teja Kuruganti, and Chad E. Duty. "Printed and hybrid electronics enabled by digital additive manufacturing technologies," Additive Manufacturing: Innovations, Advances, and Applications, eds. T. S. Srivatsan and T. S. Sudarshan. CRC Press, 131-153, 2015.

3. Yongchao Yu, Min Chen, Shutong Wang, Curtis Hill, Pooran Joshi, Teja Kuruganti, and Anming $\mathrm{Hu}$. "Laser sintering of printed anodes for Al-Air batteries," Journal of the Electrochemical Society 165, A584-A592., 2018.

4. Tolga Aytug, Matthew S. Rager, Wesley Higgins, Forrest G. Brown, Gabriel M. Veith, Christopher M. Rouleau, Hui Wang, Zachary D. Hood, Shannon M. Mahurin, Richard T. Mayes, Pooran C. Joshi, and Teja Kuruganti. "Vacuum-assisted low-temperature synthesis of reduced graphene oxide thin-film electrodes for high-performance transparent and flexible all-solid-state supercapacitors," ACS Applied Materials \& Interfaces 10(13), 11008-11017, 2018. DOI: 10.1021/acsami.8b01938 
5. Pooran Joshi, Teja Kuruganti, and Stephen Killough, "Impact of pulse thermal processing on the properties of inkjet printed metal and flexible sensors," ECS Journal of Solid State Science and Technology 4(4), 3091-3096, 2015. DOI: 10.1149/2.0161504jss

6. J. H. Noh, P. C. Joshi, T. Kuruganti, and P. D. Rack. "Pulse thermal processing for low thermal budget integration of IGZO thin film transistors," Electron Devices Society, IEEE Journal of the Electron 3(3),297-301, May 2015. DOI: 10.1109/JEDS.2014.2376411

7. Pooran Joshi, Teja Kuruganti, and Stephen Killough, "Printed, self-powered, wireless sensor platform for future smart buildings," TechConnect World Innovation Conference, Washington, DC, May 14-17, 2017.

8. Pooran Joshi, Teja Kuruganti, and Tolga Aytug. "Photonic curing for advanced thin film and device development," TMS 2017, San Diego, California, February 26-March 2, 2017.

9. Stephen M. Killough, Mohammed M. Olama, and Teja Kuruganti "Gold code-phase-shift keying: A power and bandwidth efficient communication scheme for smart buildings" 2018 IEEE International Workshop Technical Committee on Communications Quality and Reliability (CQR), Austin, Texas, 2018.

\section{COMMERCIALIZATION POSSIBILITIES AND FUTURE COLLABORATION}

- ORNL and Molex have discussed scalable manufacturing approaches for the designed wireless sensor nodes.

- Molex has the infrastructure required for sheet-to-sheet manufacturing of the nodes for commercial use.

- The team worked with building equipment manufacturers to define requirements and use-cases required for space condition monitoring and refrigeration monitoring.

- The team is currently having discussions with potential customers for the product and demonstrated the version 2 nodes at the Sensors Expo 2018 in San Jose, California.

- The team collaborated on a follow-on project to improve node communication using the LoRa network for increased interoperability.

\section{REFERENCES}

1. Buildings Energy Data Book, US Department of Energy, Office of Energy Efficiency and Renewable Energy, Washington DC,

2. F. P. Sioshansi, Energy Sustainability and the Environment, Elsevier, Oxford, UK, 2011.

3. Energy Impact of Commercial Building Controls and Performance Diagnostics: Market Characterization, Energy Impact of Building Faults and the Energy Savings Potential, TIAX Report D0180, for US DOE Contract 030400101, November 2005.

4. S. Katipamula, R. M. Underhill, J. K. Goddard, D. J. Taasevigen, M. A. Piette, J. Granderson, R. E. Brown, S. M. Lanzisera, and T. Kuruganti, Small- and Medium-Sized Commercial Building Monitoring and Controls Needs: A Scoping Study, PNNL-22169, Pacific Northwest National Laboratory, Richland, WA, 2012.

5. T. Islam, S. C. Mukhopadhyay, and N. K. Suryadevara, "Smart sensors and internet of things: A postgraduate paper," IEEE Sensors Journal, 17(3), 577-584, February 2017.

6. I. Lobachev and E. Cretu, "Smart sensor network for smart buildings," Proc. of the IEEE 7th Annual Information Technology, Electronics and Mobile Communication Conference (IEMCON), October 2016.

7. C. Jacquemod, B. Nicolle, and G. Jacquemod, "WSN for smart building application," Proc. of the 10th European Workshop on Microelectronics Education (EWME), May 2014.

8. R. Das and P. Harrop, Printed, Organic and Flexible Electronics Forecasts, Players and Opportunities 2011-2021, IDTechEx Business Report, 2011. 
9. “Commercial Buildings Energy Consumption Survey,” Energy Information Administration, Department of Energy, Washington, DC, 2009.

10. F. Robol, F. Viani, E. Giarola, and A. Massa, "Wireless sensors for distributed monitoring of energy-efficient smart buildings," Proc. of the IEEE 15th Mediterranean Microwave Symposium (MMS), December 2015.

11. W. Wang, N. Wang, E. Jafer, M. Hayes, B. O’Flynn, and C. O’Mathuna, “Autonomous wireless sensor network-based building energy and environment monitoring system design," Proc. of the International Conference on Environmental Science and Information Application Technology (ESIAT), pp. 367-372, July 2010.

12. N. K. Suryadevara, S. C. Mukhopadhyay, S. Dieter, T. Kelly, and S. P. S. Gill, "WSN-based smart sensors and actuator for power management in intelligent buildings," IEEE/ASME Transactions on Mechatronics, 20(2), 564-571, April 2015.

13. O. R. Baiocchi, G. Mobus, F. B. S. D. Carvalho, R. M. Bacurau, and S. A. F. Soares, "Development of a smart building wireless sensors network: Cooperation between University of Washington Tacoma and Brazilian universities," Proc. of the IEEE Frontiers in Education Conference, October 2013.

14. J. Shaha and B. Mishraa, "Customized IoT enabled wireless sensing and monitoring platform for smart buildings," Procedia Technology, 23, 256-263, 2016.

15. M. A. Benatia, A. Louis, and D. Baudry, "WSN's modeling for a smart building application," Proc. of the IEEE International Energy Conference (ENERGYCON), pp. 821-827, July 2014.

16. A. G. Pena, M. L. Boucheret, C. Macabiau, J. L. Damidaux, L. Ries, S. Corazza, and A. C. Escher, "Implementation of code shift keying signaling technique in GALILEO E1 signal," Proc. of the 5th ESA Workshop on Satellite Navigation Technologies and European Workshop on GNSS Signals and Signal Processing (NAVITEC), December 2010.

17. P. Harrop, V. Zhitomirsky, and F. Gonzalez, Electrochemical Double Layer Capacitors: Supercapacitors 2014-2024, IDTechEx Business Report.

18. Y. R. Tsai, "M-ary spreading code phase shift keying modulation for DSSS multiple access systems," IEEE Transactions on Communications, 57(11), 3220-3224, November 2009.

19. G. M. Dillard, M. Reuter, J. Zeidler, and B. Zeidler, "Cyclic code shift keying: A low probability of intercept communication technique," IEEE Transactions on Aerospace and Electronic Systems, 39(3), 786-798, July 2003.

20. A. Y. C. Wong and V. C. M. Leung, "Code-phase-shift keying: A power and bandwidth efficient spread spectrum signaling technique for wireless local area network applications," Proc. of the IEEE Canadian Conference on Electrical and Computer Engineering, pp. 478-481, May 1997.

21. M. M. Olama, S. M. Killough, T. Kuruganti, and T. E. Carroll, "Design, implementation, and evaluation of a hybrid DS/FFH spread-spectrum radio transceiver," Proc. of the IEEE Military Communication Conference (MILCOM'14), October 2014.

22. T. J. Grant, "Network topology in command and control. Advances in information security, privacy, and ethics," IGI Global, pp. 228-250, 2014.

23. S. M. Ross, Stochastic Processes, Wiley, 1996.

24. J. Anthes, "OOK, ASK and FSK modulation in the presence of an interfering signal," Murata Electronics North America, Carrollton, Texas, 2007.

25. D. H. Mudarri, Building Codes and Indoor Air Quality, prepared for the US Environmental Protection Agency, Office of Radiation and Indoor Air, Indoor Environments Division, by the Cadmus Group, Arlington, VA, September 2010.

26. Energy Standard for Buildings except Low-rise Residential Buildings, ANSI/ASHRAE/IES Standard 90.1-2010, 2010 Available from ASHRAE at https://www.ashrae.org/.

27. 2012 International Energy Conservation Code, International Code Council, 2012. Available from the International Code Council at http://www.iccsafe.org/.

28. Energy-efficient Design of Low-rise Residential Buildings, ANSI/ASHRAE Standard 90.2-2007, 2007. Available from ASHRAE at https://www.ashrae.org/. 
29. 2012 International Residential Code, International Code Council, 2012. Available from International Code Council at http://www.iccsafe.org/.

30. NFPA 5000: Building Construction and Safety Code, National Fire Protection Association, 2012. Available from the National Fire Protection Association at http://www.nfpa.org/.

31. Thermal Environmental Conditions for Human Occupancy, ANSI/ASHRAE Standard 55-2010, 2010. Available from ASHRAE at https://www.ashrae.org/.

32. M. Hydeman, "Implications of current thermal guidelines for data center energy use," ASHRAE Journal, August, 30-36, 2010.

33. Telecommunications Infrastructure Standard for Data Centers, ANSI/TIA-942-1, developed by Telecommunications Industry Association, 2005.

34. Ventilation for Acceptable Indoor Air Quality, ANSI/ASHRAE Standard 62-2001, ANSI and ASHRAE, 2001. Available from ASHRAE at https://www.ashrae.org/.

35. M. Jouaneh, Codes and Controls: Commercial Building Energy Code Requirements for Light Control, Lutron, 2011. Available at www.dominionelectric.com/content/ ASHRAE\%202010\%20Requirements.pdf

36. D. Wang, E. Arens, T. Webster, and M. Shi, "How the number and placement of sensors controlling room air distribution systems affect energy use and comfort," ESL-IC-02-10-21, Proc. of the Second International Conference for Enhanced Building Operations, Richardson, Texas, October 14-18, 2002.

37. X. Liu, B. Akinci, J. H. Garrett, and O. Akin, "Requirements for a computerized approach to plan sensor placement in the HVAC systems," Proc. of the International Conference on Computing in Civil and Building Engineering, June 30-July 2, 2010, Nottingham University Press, W. Tizani, ed., no. 118, p. 235, 2010.

38. Standard for the Design of High-performance Green Buildings, ANSI/ASHRAE/USGBC/IES Standard 189.1-2011, ANSI, ASHRAE, US Green Building Council, and IES, 2011. Available from ASHRAE at https://www.ashrae.org/. 


\title{
APPENDIX A. LOW-COST WIRELESS SENSORS (MOLEX) TEST DEPLOYMENT
}

\author{
EXECUTIVE SUMMARY
}

ORNL has developed a prototype low-cost wireless sensor in collaboration with Molex. The sensor node is designed to meet requirements for measuring building space conditions. It measures temperature, humidity, and light and transmits the data at low sample rates such as 10 minutes. The prototype peel-and stick wireless sensor is manufactured on a thin film with a photovoltaic cell powering a rechargeable battery. It also includes integrated circuitry for sensor signal processing, on-board computation, and wireless communication.

Thirty-nine sensor tags were deployed in Flexible Research Platforms \#1 and \#2 (FRP\#1) (FRP\#2) at ORNL in Oak Ridge, Tennessee, in June and July in a variety of conditioned spaces, unconditioned spaces, a low-temperature refrigeration case, and medium-temperature refrigeration case. The sensor data were compared with building instrumentation to validate that the sensor measurements have appropriate accuracy for building applications. Sensor tags were compared with research thermocouples and were found to be within $0.5^{\circ} \mathrm{F}$ of the thermocouple readings. Sensor tag humidity measurements were compared with research instrumentation and were found to be consistently 5-9\% lower than the research instrumentation. The sensor tag luminosity (lights on/off) measurement was found to correlate with the building lighting schedule. The sensors were exposed to temperatures ranging from $-5^{\circ} \mathrm{F}$ (lowtemperature refrigeration case in FRP\#1) to $90^{\circ} \mathrm{F}$ (unconditioned stairwell in FRP\#2). The receiver system successfully collected transmission data and provided those data to the collection computing device. The testing on FRP\#1 and FRP\#2 will continue to provide more experience and data for additional comparisons with building instrumentation, additional wireless data transmission study, and deployment maturation.

ORNL is planning field deployment tests with commercial partners. Emerson Climate Technologies, Pilot Flying J Travel Centers, and Home Depot are all planning field tests in commercial sites or testing laboratories starting in August 2018. ORNL is developing an update to the computing device to incorporate a low-cost single-board computing device to collect the data in an embedded manner to simplify the data collection system and process.

\section{INTRODUCTION}

\section{BACKGROUND}

ORNL has developed a prototype low cost wireless sensor (Figure 55) in collaboration with Molex. The prototype peel-and stick wireless sensor is $4.75 \times 3 \times 0.23$ in. and is manufactured on a thin film with a photovoltaic cell powering a rechargeable battery. It also includes integrated circuitry for sensor signal processing, on-board computation, and wireless communication. The sensor node is designed to meet requirements for measuring building space conditions. It measures temperature, humidity, and light and transfers the data at low sample rates (e.g., 10 minutes). 


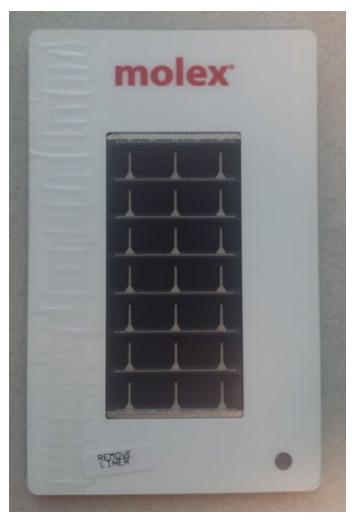

Figure 55. Initial prototype of energy harvesting wireless sensor.

\section{DEPLOYMENT ARCHITECTURE}

ORNL developed a deployment architecture that consists of an RF receiver device that provides the received measurement data to a computing device for collection (Figure 56). The sensor receiver hardware is shown in Figure 57. The sensor receiver consists of an RF processing board that converts the 433.92 MHz signal to baseband, and a Xilinx Zynq 7020 FPGA does the spread-spectrum decoding of the signal. The spread-spectrum decoding processes the 1023 bit package to deliver 20 bit data (51:1 expansion). The Xilinx Zynq FPGA combines FGPA hardware programming with an ARM-based processor to support software programming.

The ORNL team used the Node-Red programming tool to develop an application for the computing device to collect the receiver data. Node-Red is a browser-based interface that logs all data output by the receiver and displays desired data to the user. When the sensor receiver receives a transmission message from a Molex tag, the data is passed via TCP/IP to the Node-Red application on the computing device. The application on the computing device then logs this new input in a MongoDB database. Meanwhile, the customer can configure the user interface to create "rooms" with corresponding Molex tag data displayed. When the application logs new data into the database from the receiver, the display will update the corresponding tag entry with the newly received information. Initially, a laptop was used as the computing device. Later, other platforms such as single-board low-cost computing devices will be used. 

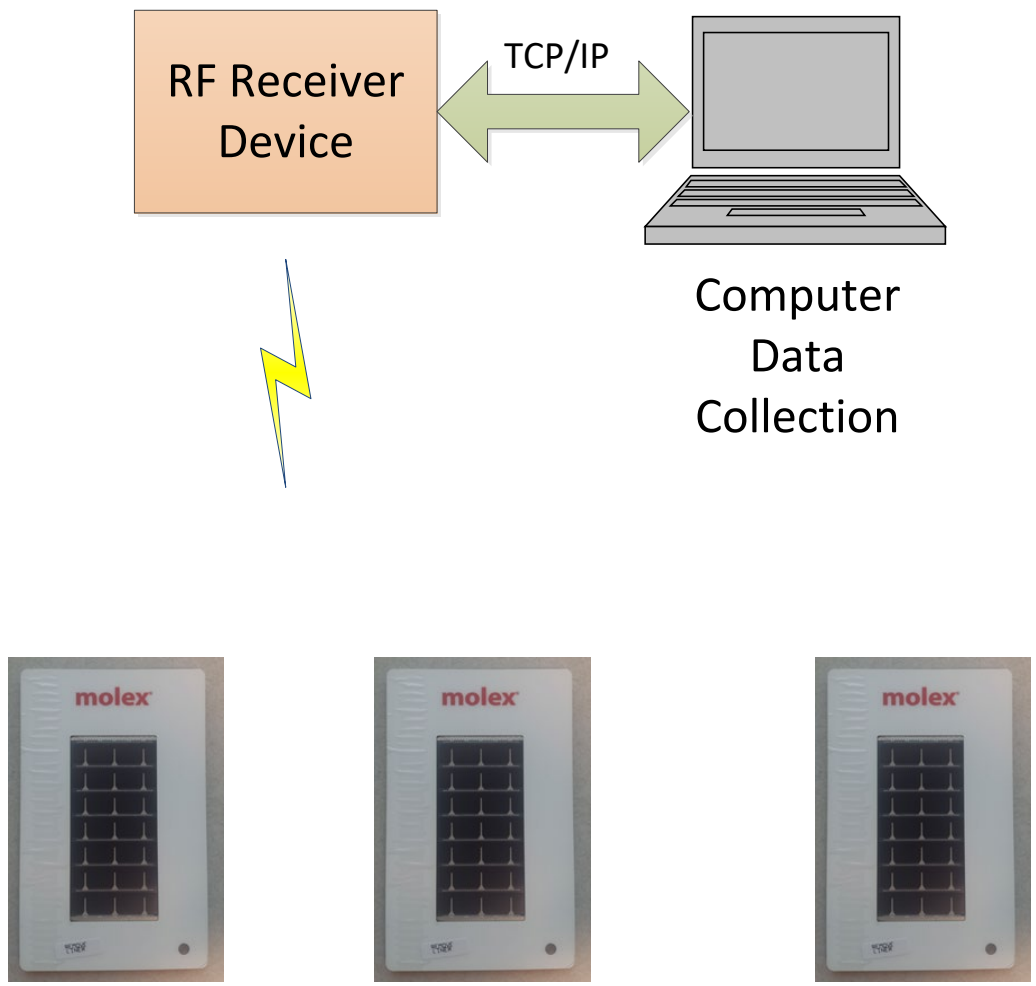

Figure 56 Deployment architecture.

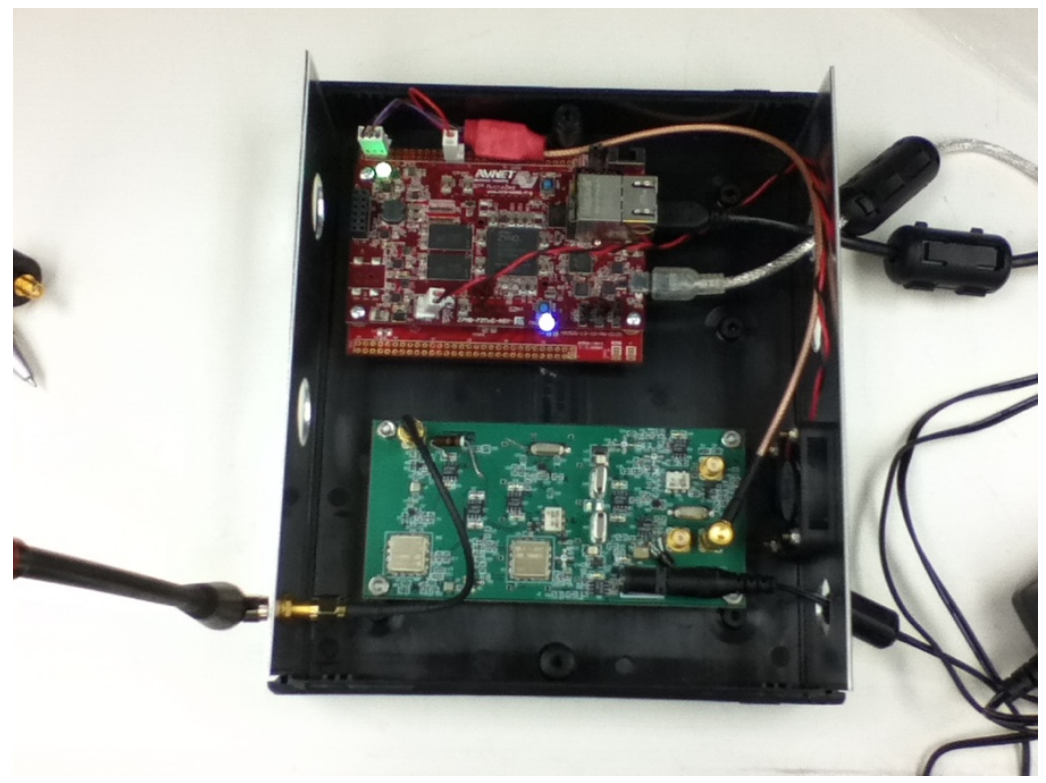

Figure 57 Sensor receiver. 


\section{SENSOR CONFIGURATION}

The target measurement temperature range for space conditioning is $60-90^{\circ} \mathrm{F}$ and for refrigeration is -20 to $45^{\circ} \mathrm{F}$. The current wireless transmission data design represents the measured temperature using a 7 bit data type. The actual analog measurement on the sensor is encoded to this 7 bit data type for transmission, and the receiver will decode this value into the actual measurement. This approach led to the development of a custom temperature scale to balance the desired temperature measurement precision in ranges of interest with the available 7 bit data resolution. Figure 58 illustrates the selection of different levels of precision, such as $1^{\circ} \mathrm{F}$ or $0.5^{\circ} \mathrm{F}$ resolution versus the measurement range. The left y-axis in Figure 58 displays the temperature range of "Scheme 2 " versus the $\mathrm{x}$-axis 7 bit measurement values. The right $y$ axis in Figure 58 illustrates the measurement prevision varying as desired over the measurement range.

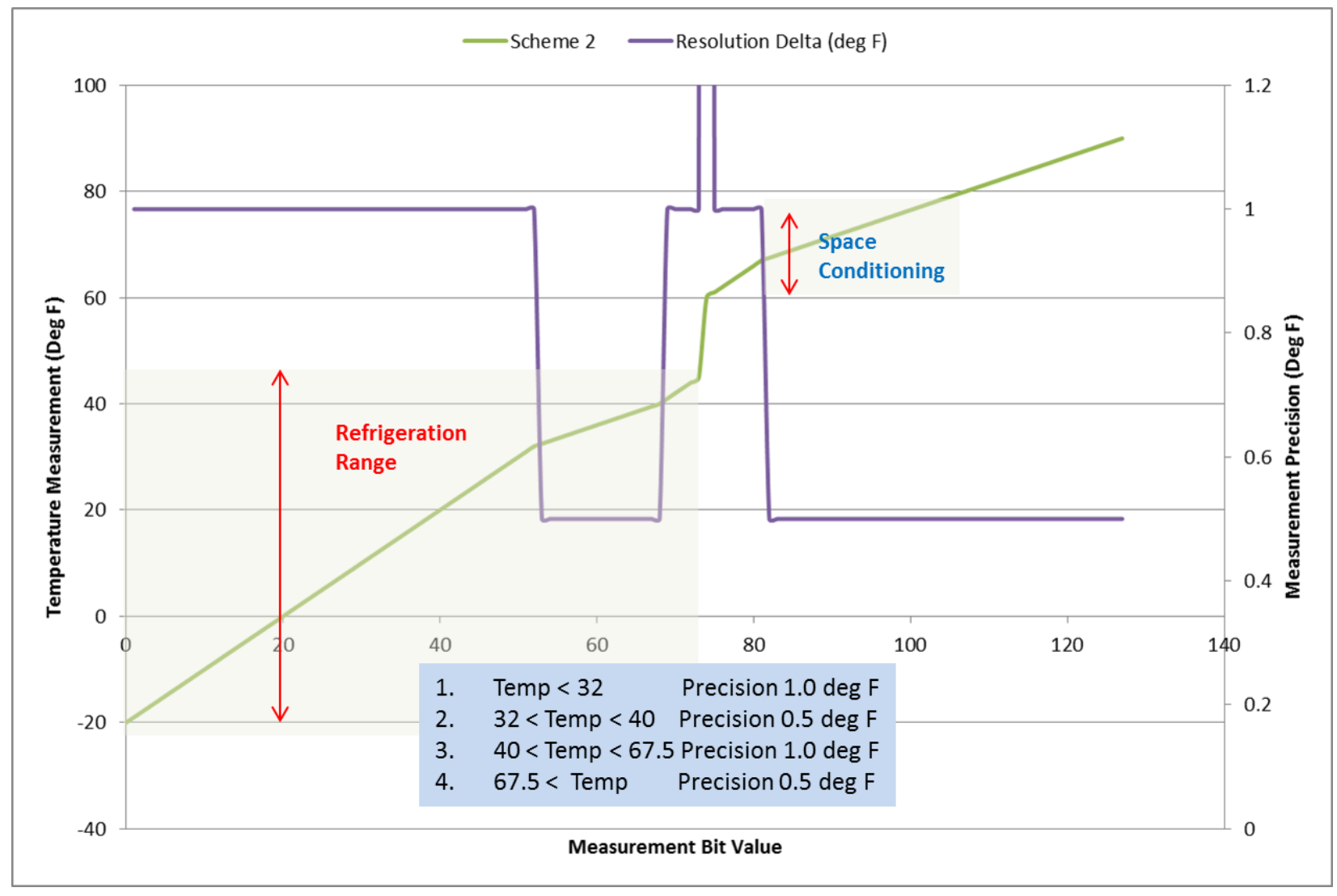

Figure 58 Temperature measurement data scale.

The energy storage for the sensors consists of either a rechargeable CR2032 battery or a supercapacitor and a nonrechargeable battery. The sensors that are intended for space temperature were configured with the rechargeable CR2032 battery, and the sensors that are intended for refrigeration measurements were configured with a supercapacitor and a nonrechargeable battery.

The sensors were configured to sample and transmit every 90 seconds during lights-on periods and every 22 minutes for lights-off conditions for the deployment testing. The sensors transmit a data package that includes the address header (to identify a specific building), sensor tag number, temperature measurement, humidity measurement, and light-level luminosity measurement for lights-on or lights-off conditions. Each sensor must be configured individually for the specific address header and sensor tag 
number. The custom temperature scale must be programmed into the sensor and into the receiver to enable the accurate encoding and decoding of the temperature measurement.

\section{FRP\#1 TEST DEPLOYMENT}

Twenty sensors were deployed in FRP\#1 in June. The sensor locations for the 11 space-conditioning sensors are shown in Figure 59. The sensors were located between 4 and 5 feet off the floor. The "RX" item in Figure 59 is the receiver location. See Figure 60 for a photograph of the receiver and data collection laptop. Nine sensors were deployed in refrigeration cases for a $\mathrm{CO}_{2}$ refrigerant research system in FRP\#1 (Figure 61). The locations of the sensors in the low-temperature case (frozen) are shown in Figure 62 and the locations of the sensors in the medium temperature case (above freezing) in Figure 63. Some photographs of installed sensors are show in Figure 64 - Figure 67. All 20 of the FRP\#1 sensors transmitted data successfully. Analysis of the measurement data is discussed in the next section.

\begin{tabular}{|c|c|}
\hline $\begin{array}{l}\text { Sensor } \\
\#\end{array}$ & Location \\
\hline 1 & Right Side of East Door \\
\hline 2 & East Thermostat \\
\hline 3 & West Thermostat \\
\hline 4 & $\begin{array}{l}\text { North West Corner, Near NW } \\
\text { Door }\end{array}$ \\
\hline 5 & $\begin{array}{l}\text { NW Quadrant Thermocouple } \\
\text { Stack }\end{array}$ \\
\hline 6 & $\begin{array}{l}\text { SW Quadrant Thermocouple } \\
\text { Stack }\end{array}$ \\
\hline 7 & South West Corner \\
\hline 8 & South Center Wall \\
\hline 9 & South West Corner \\
\hline 10 & South East Corner \\
\hline 20 & North East Corner \\
\hline
\end{tabular}

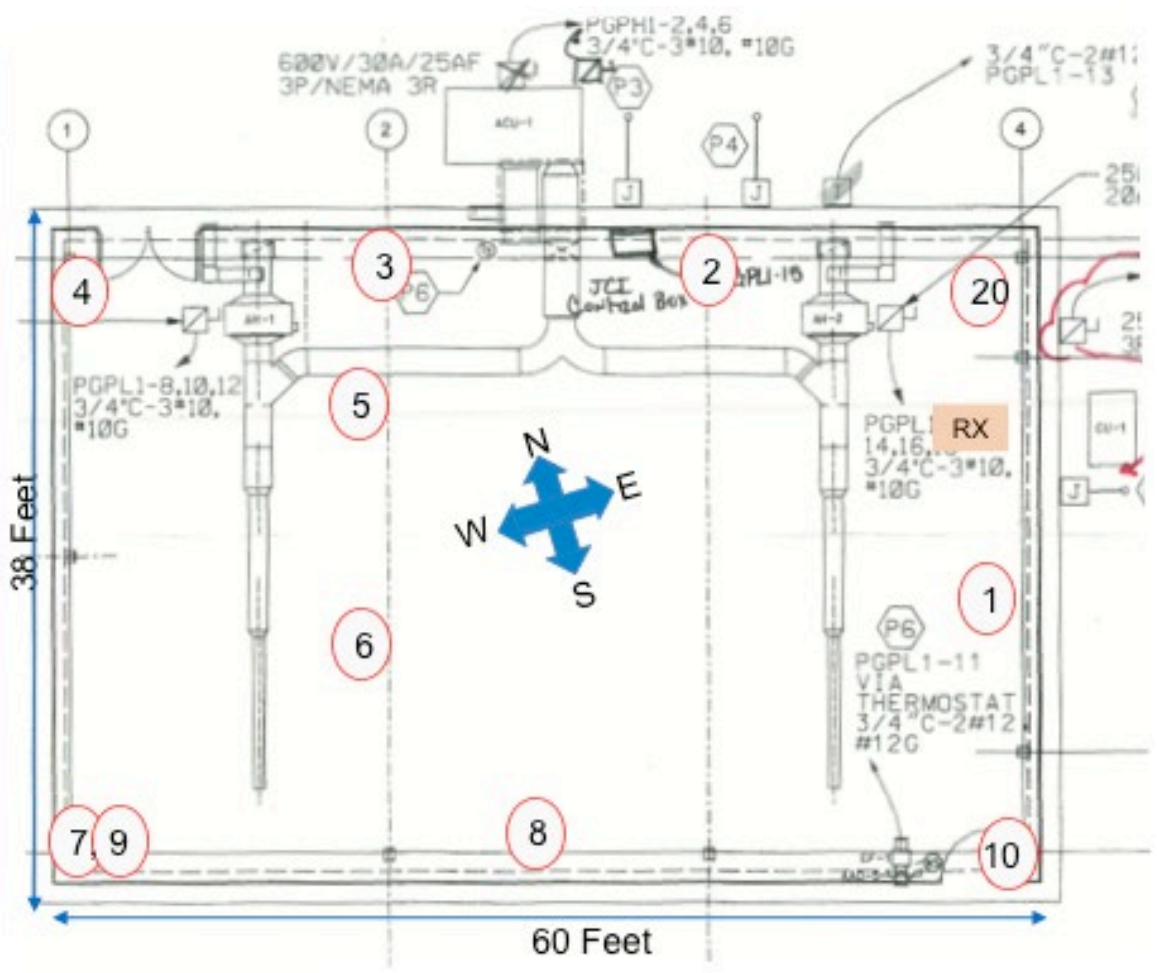

Figure 59 FRP\#1 space temperature measurement sensor locations. 


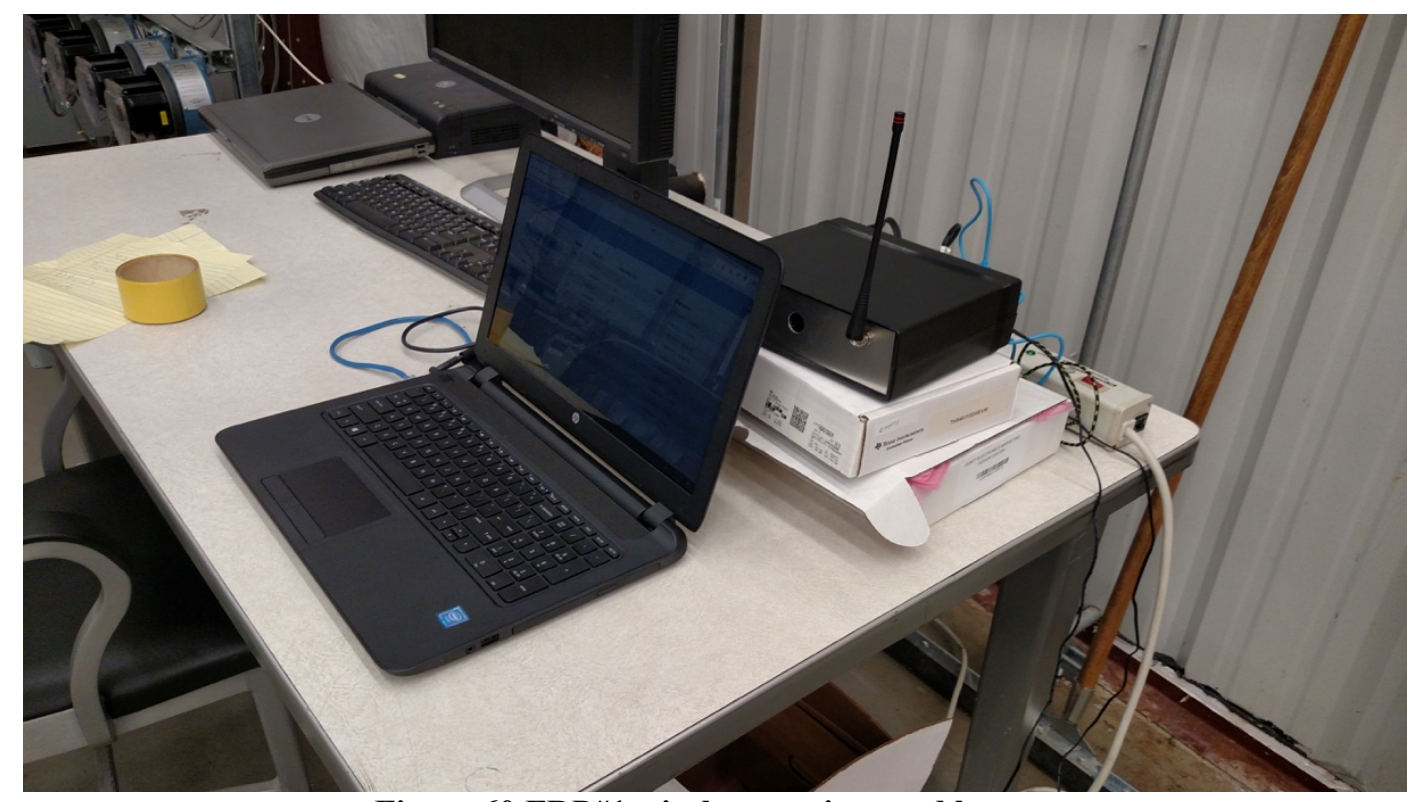

Figure 60 FRP\#1 wireless receiver and laptop.

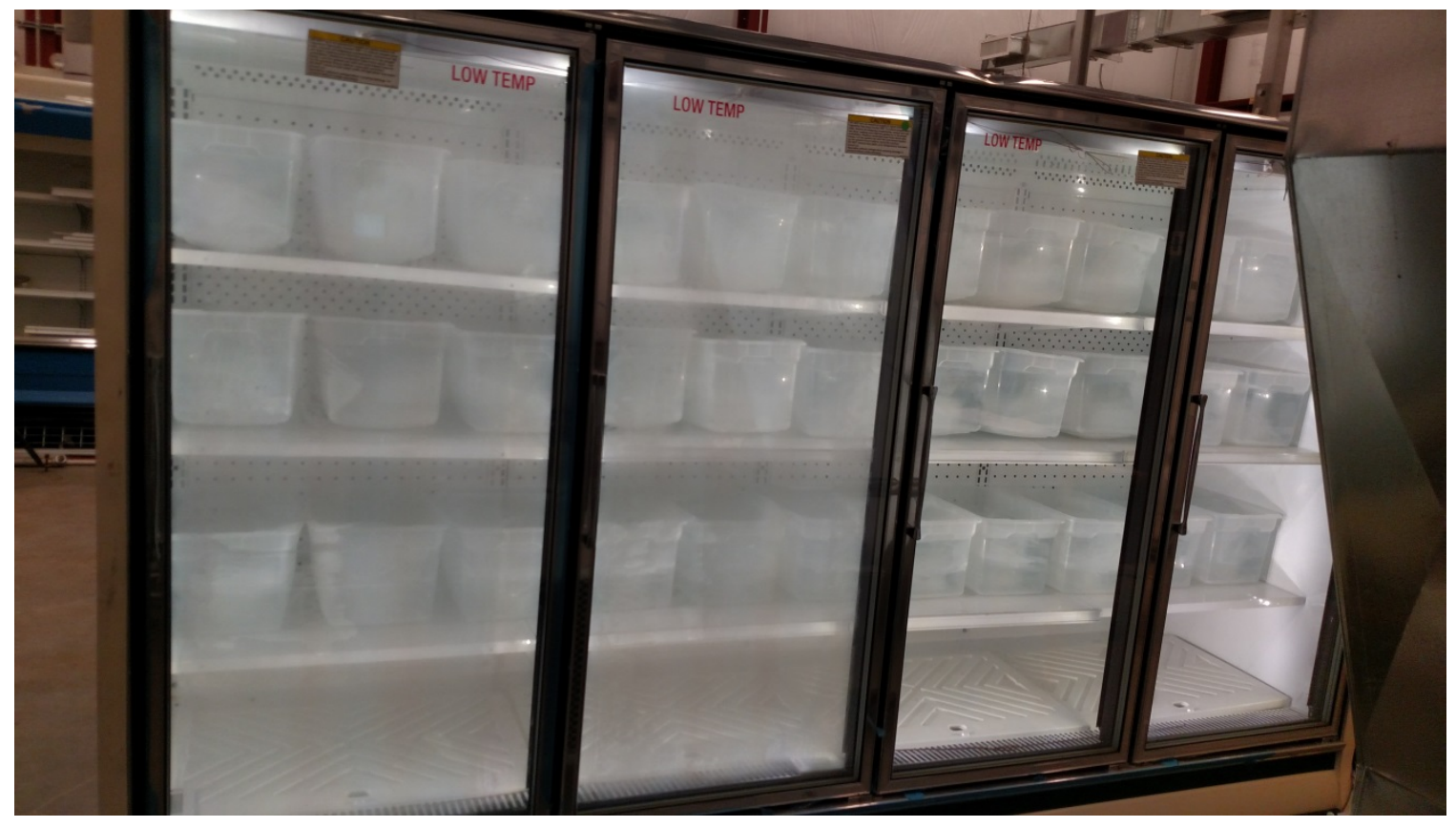

Figure 61 FRP\#1 CO2 refrigeration system. 


\begin{tabular}{|l|l|}
\hline $\begin{array}{l}\text { Sensor } \\
\#\end{array}$ & Location \\
\hline 11 & LT - Bottom Center \\
\hline 12 & LT - Top Center \\
\hline 13 & LT - Bottom Left \\
\hline 14 & LT - Top Left \\
\hline 15 & MT - Bottom Center \\
\hline 16 & MT - Top Center \\
\hline 17 & MT - Top Center Outside \\
\hline 18 & MT - Bottom Left \\
\hline 19 & MT - Top Left \\
\hline
\end{tabular}

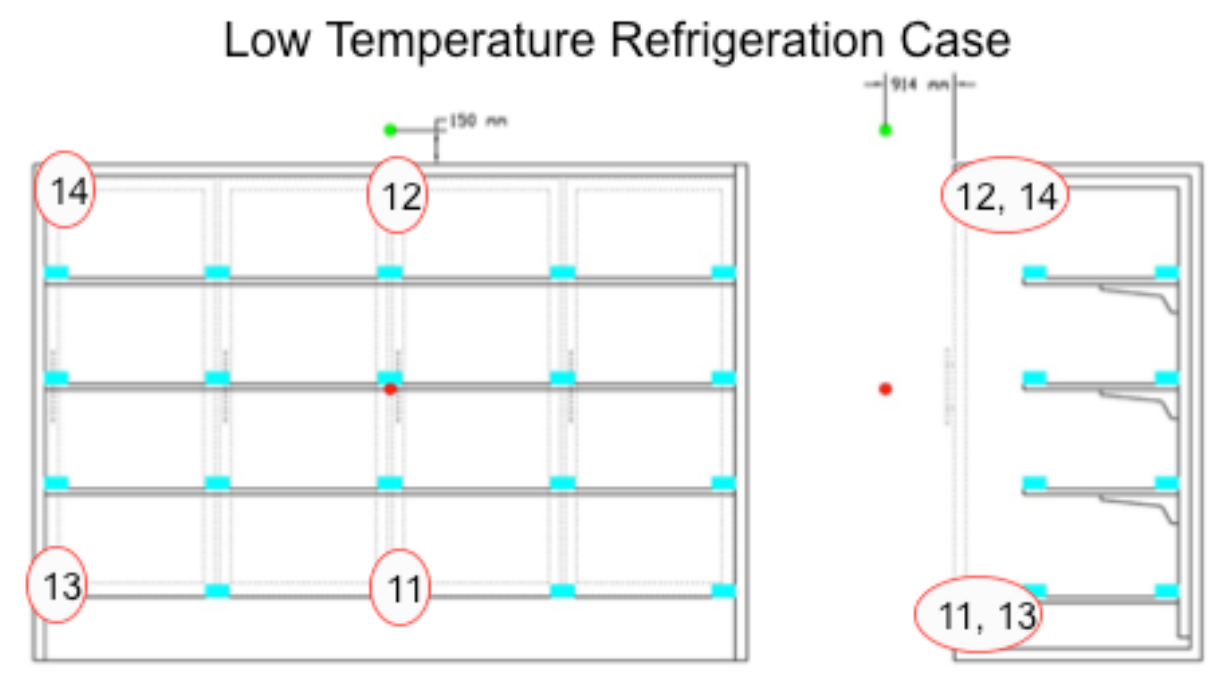

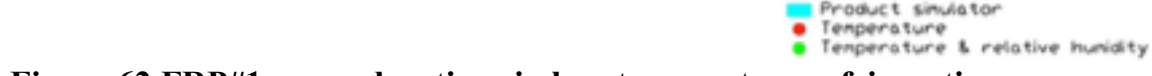

Figure 62 FRP\#1 sensor locations in low-temperature refrigeration case.

\begin{tabular}{|l|l|}
\hline $\begin{array}{l}\text { Sensor } \\
\#\end{array}$ & Location \\
\hline 11 & LT - Bottom Center \\
\hline 12 & LT - Top Center \\
\hline 13 & LT - Bottom Left \\
\hline 14 & LT - Top Left \\
\hline 15 & MT - Bottom Center \\
\hline 16 & MT - Top Center \\
\hline 17 & MT - Top Center Outside \\
\hline 18 & MT - Bottom Left \\
\hline 19 & MT - Top Left \\
\hline
\end{tabular}

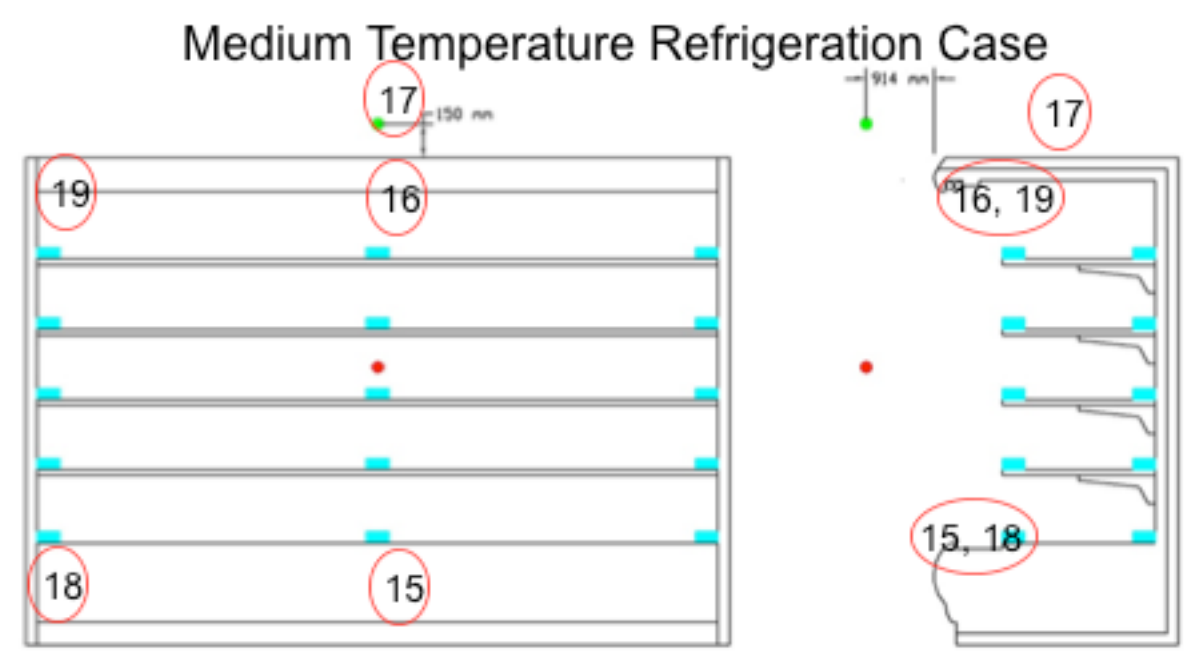

Figure 63 FRP\#1 sensor locations m medium-temperature refrigeration case. 


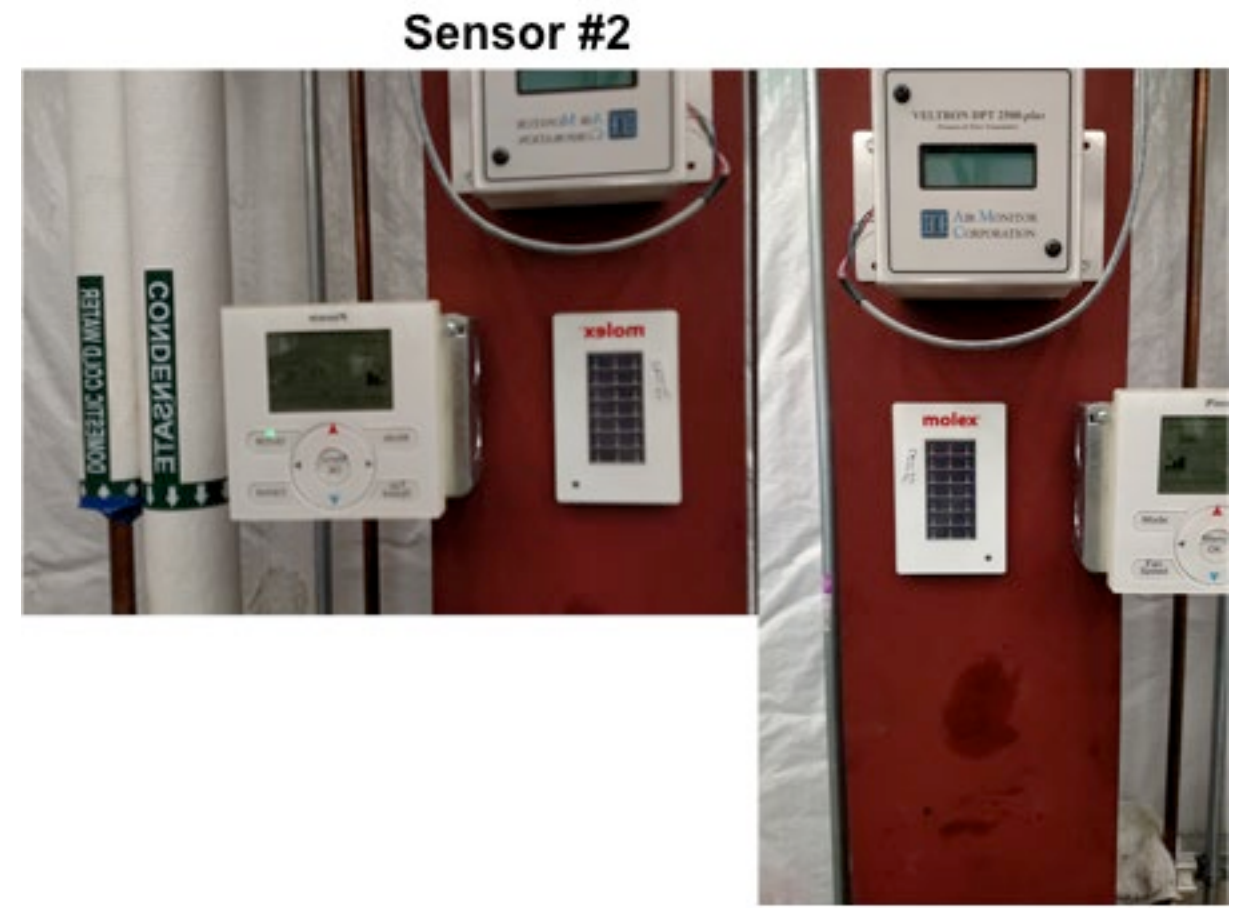

Figure 64 FRP\#1 sensor \#2.

\section{Sensor \#10}

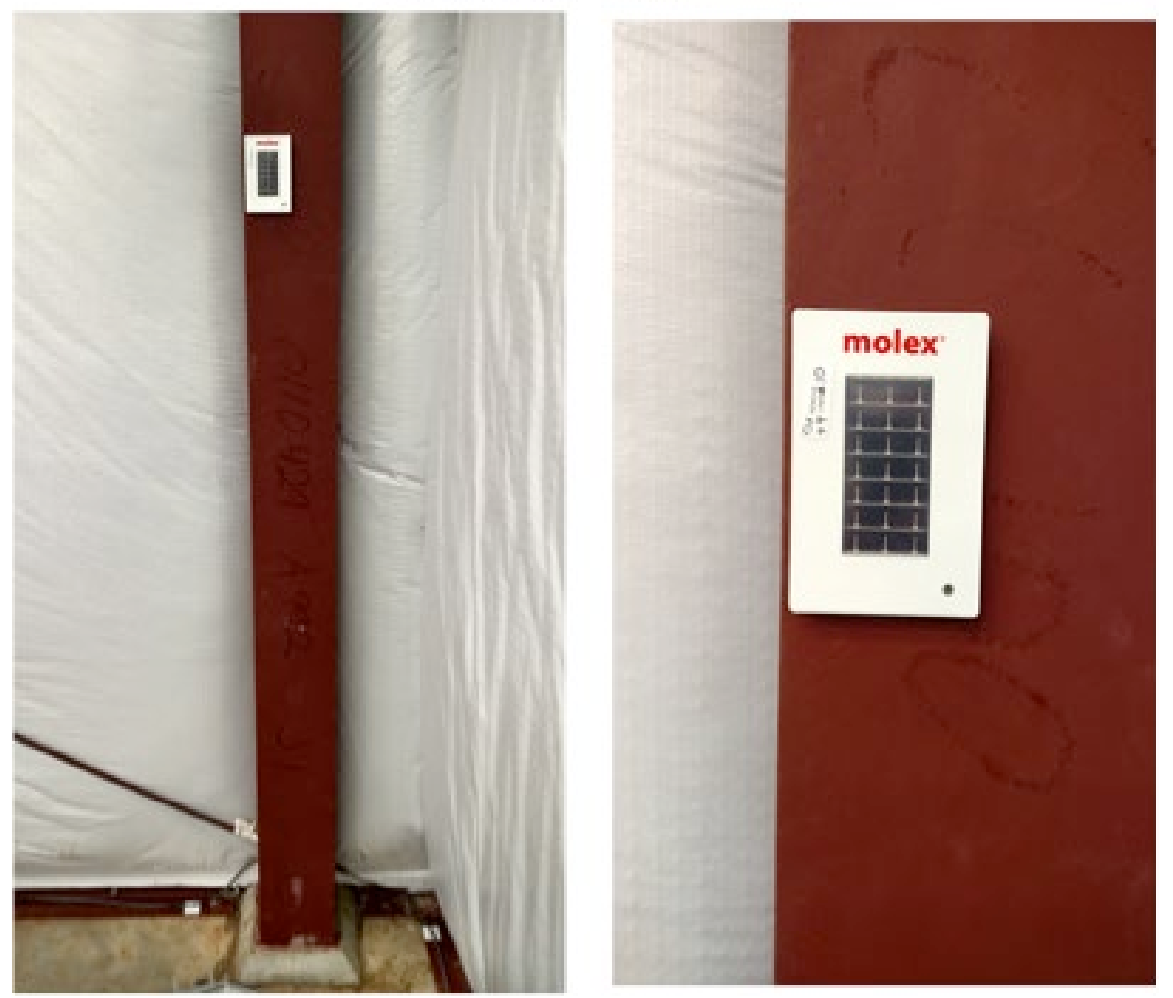

Figure 65 FRP\#1 sensor \#10. 
Low Temperature Refrigeration Case

Sensor \#12

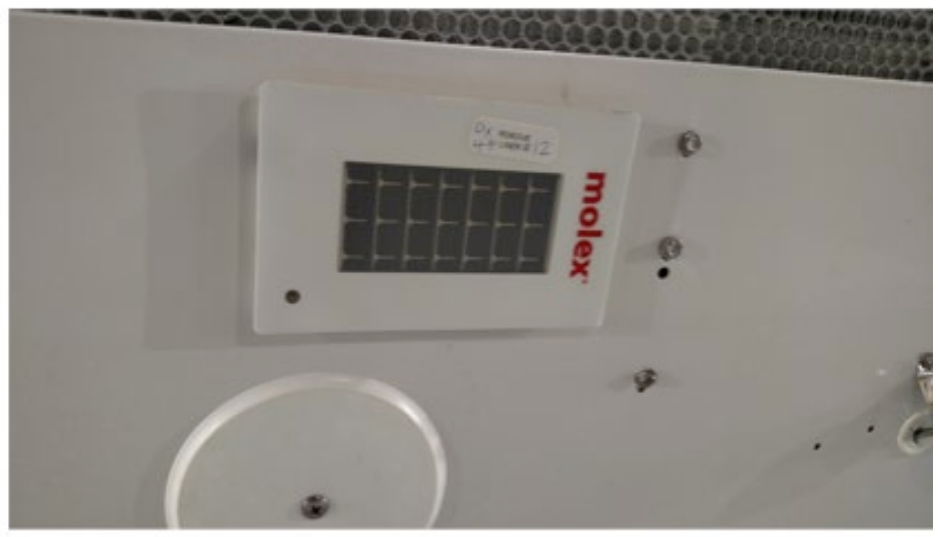

Figure 66 FRP\#1 sensor \#10.

\section{Medium Temperature Refrigeration Case} Sensor \#19

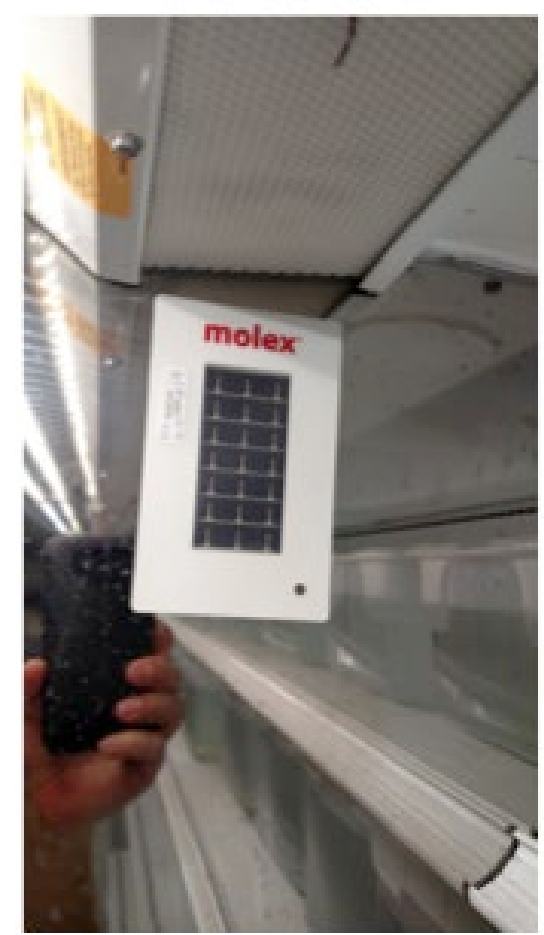

Figure 67 FRP\#1 sensor \#19. 


\section{FRP\#1 TEST DEPLOYMENT DATA ANALYSIS}

Data were collected from the FRP\#1 test and analysis was performed to quantify the sensor test performance. The analysis in this report contains data from June 30 to July 19. Figure 68 illustrates space temperature measurements over the time period for sensors \#1 through \#10. Figure 69 and Figure 70 compare sensor tags with research instrumentation for accuracy comparison purposes. The comparison indicates that the Molex sensors \#5 and \#6 space temperature measurements are within $0.5^{\circ} \mathrm{F}$ of the research thermocouples. Figure 71 illustrates the performance of sensors \#1 through \#10 for humidity measurements. Figure 72 compares sensor tag humidity measurements to research instrumentation, which indicates that the tag sensor RH measurements are consistently 5-9\% lower than the research instrumentation. Figure 73 shows that the sensor tags luminosity (lights on/off) measurement is measuring the building light scheduled on/off sequence. Figure 74 compares the luminosity closely with the building light schedule to demonstrate proper operation of the light measurement.

Sensors \#11 through \#20 were located either in the refrigeration cases (\#11 - \#16, \#18 - \#19) or near the refrigeration cases (\#17 and \#20). Sensor tags \#11 through \#14 were located inside the low-temperature refrigeration case. Figure 75 and Figure 76 illustrate the temperature measurements for sensor tags \#11 \#14. The low-temperature case refrigeration set point is $-5^{\circ} \mathrm{F}$. The periodic defrost events are easily observed in the measurement data.

Sensors \#15, \#16, \#18, and \#19 were located in the medium-temperature refrigeration case. The case air set point was $38^{\circ} \mathrm{F}$. Figure 77 and Figure 78 illustrate the sensor tag temperature measurements in the medium-temperature refrigeration case. Figure 79 illustrates the humidity measurements in the mediumand low-temperature refrigeration cases. Note that the medium-temperature case is an open case, which will have high humidity levels in the warm weather; the low-temperature case will have frozen moisture present, which will limit the ability of the sensor to accurately measure the local air humidity conditions. Figure 80 illustrates the luminosity (lights on/off) measurements for the cases that have lights always on.

The sensors operate with different sample and transmit rates, based on the lighting levels, to conserve battery power at times when the solar cell is not charging the battery. If the lights are on, the sensor transmits every 90 seconds. When the lights are not on, the sensor transmits every 22 minutes. Figure 81 illustrates the sensor varying the transmit rate during lights-on and lights-off periods for sensor tag \#1. Figure 82 illustrates that the transmit data rate for sensor tag \#11, which is located in the low-temperature refrigeration case, is consistently at a faster rate because the refrigeration case lights are always on. Figure 83 compares the sensor transmit rate with the luminosity (lights on/off) measurement to illustrate the behavior relationship of data transmissions and light levels. Figure 84 illustrates the data transmission quantities for the different sensor locations. These data transmission rates are directly related to the transmission rates shown in Figure 81 and Figure 83. Some anomalous data were observed at very low occurrence rates from the various sensors. This issue is being investigated by the team. 


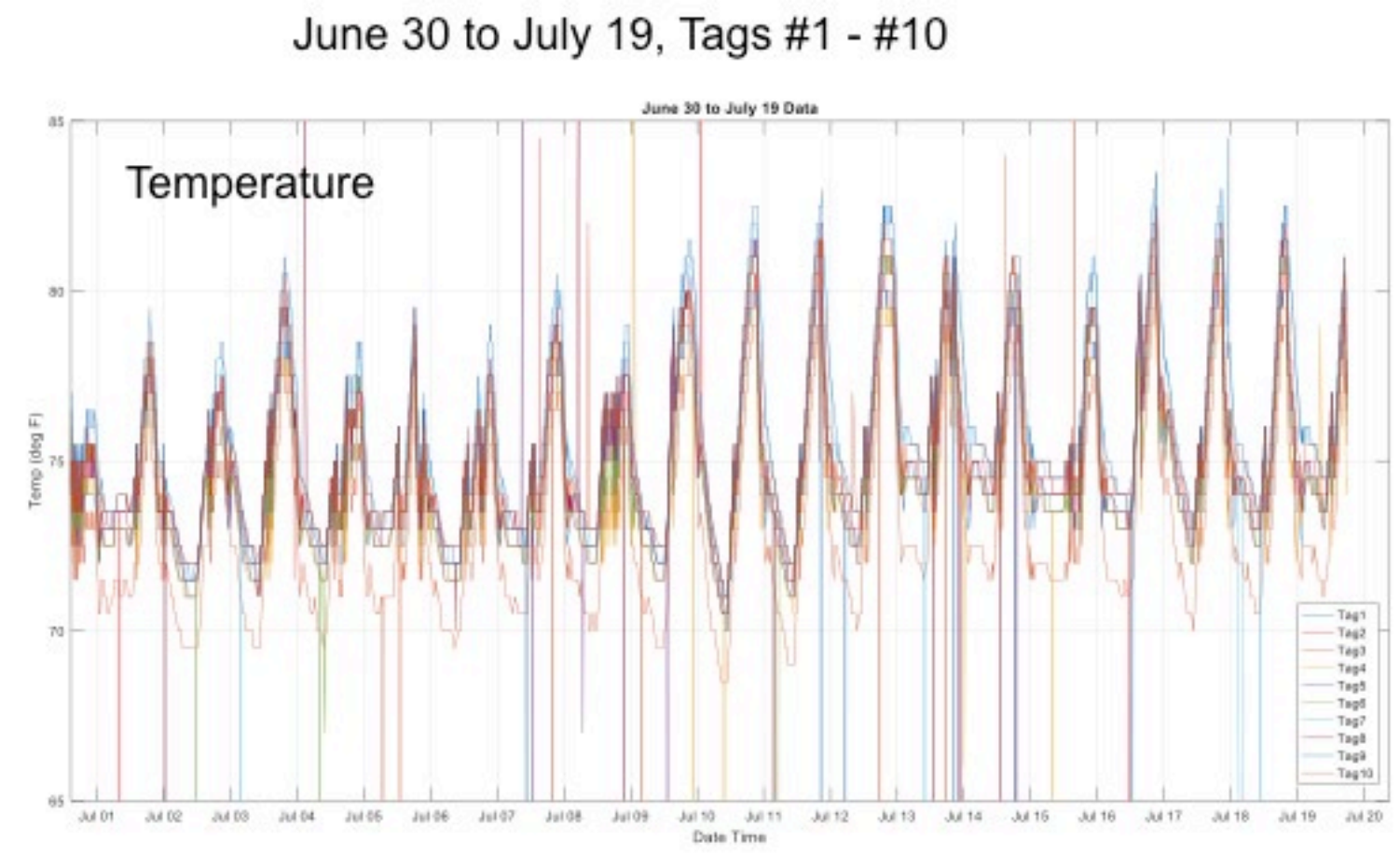

Figure 68 FRP\#1 sensor tags \#1 - \#10 temperature measurement June 30 to July 19.

Tags \# 5 Compared with Local Thermocouple T1_AMBNW

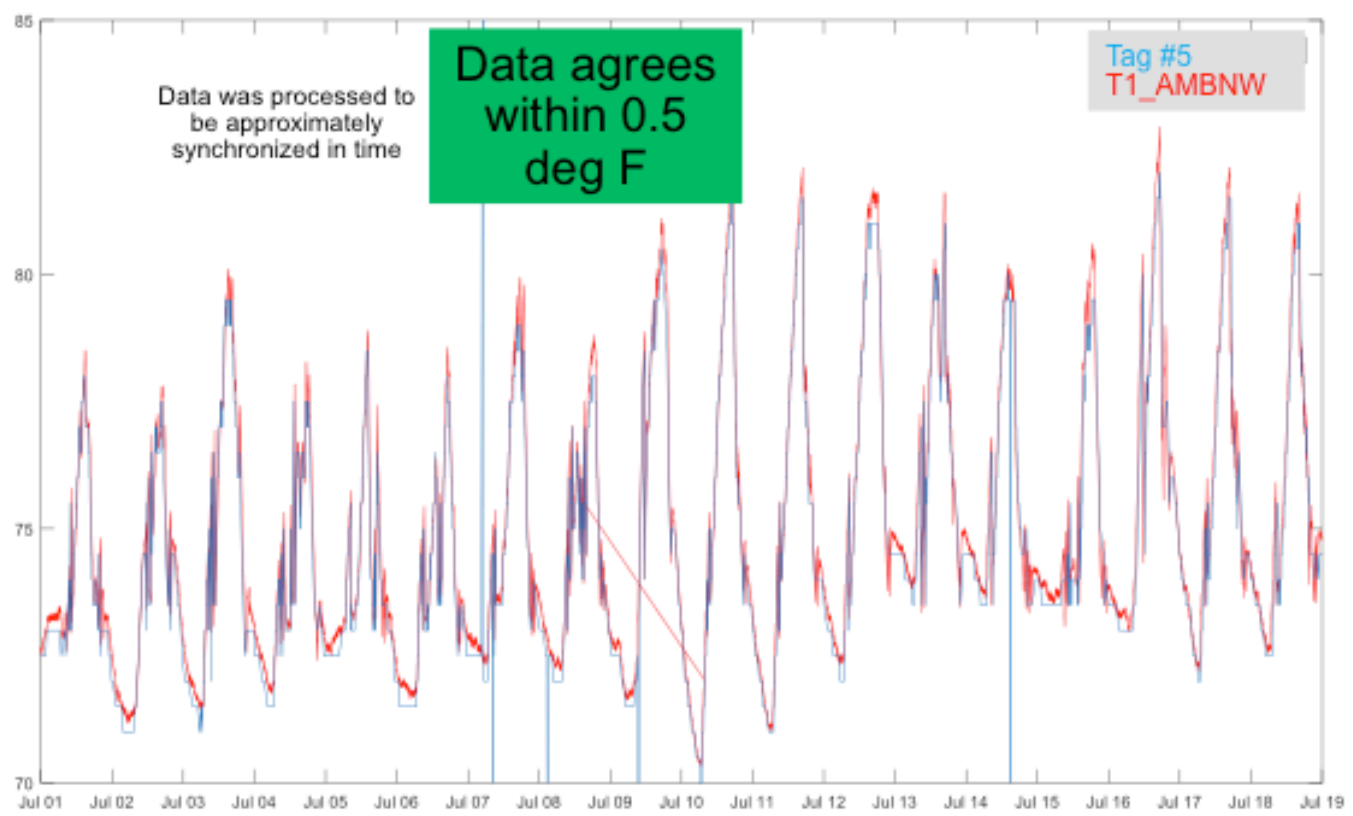

Figure 69 FRP\#1 sensor tag \#5 temperature measurement compared with research thermocouple 
Tags \# 6 Compared with Local Thermocouple T1_AMBSW

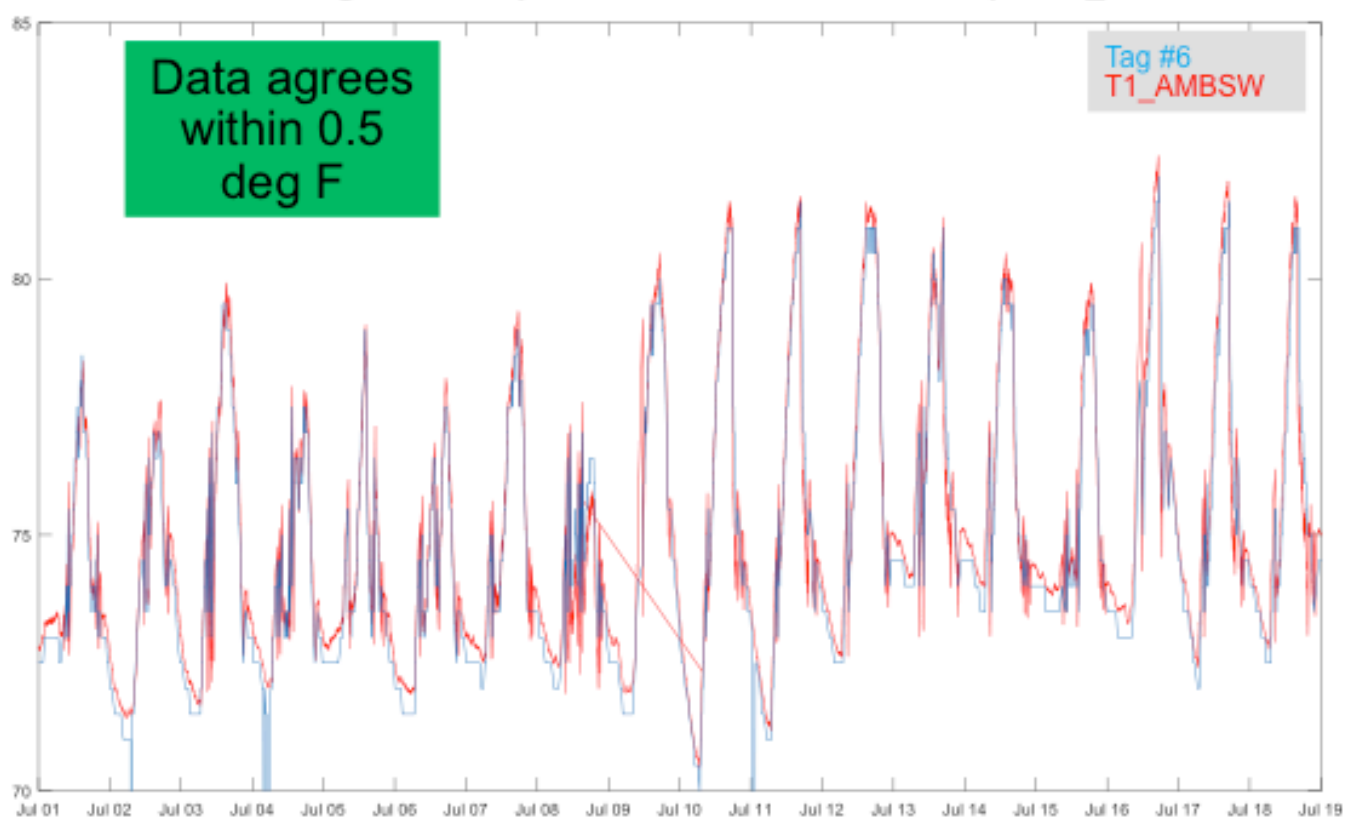

Figure 70 FRP\#1 sensor tag \#6 temperature measurement compared with research thermocouple

June 30 to July 19, Tags \#1 - \#10

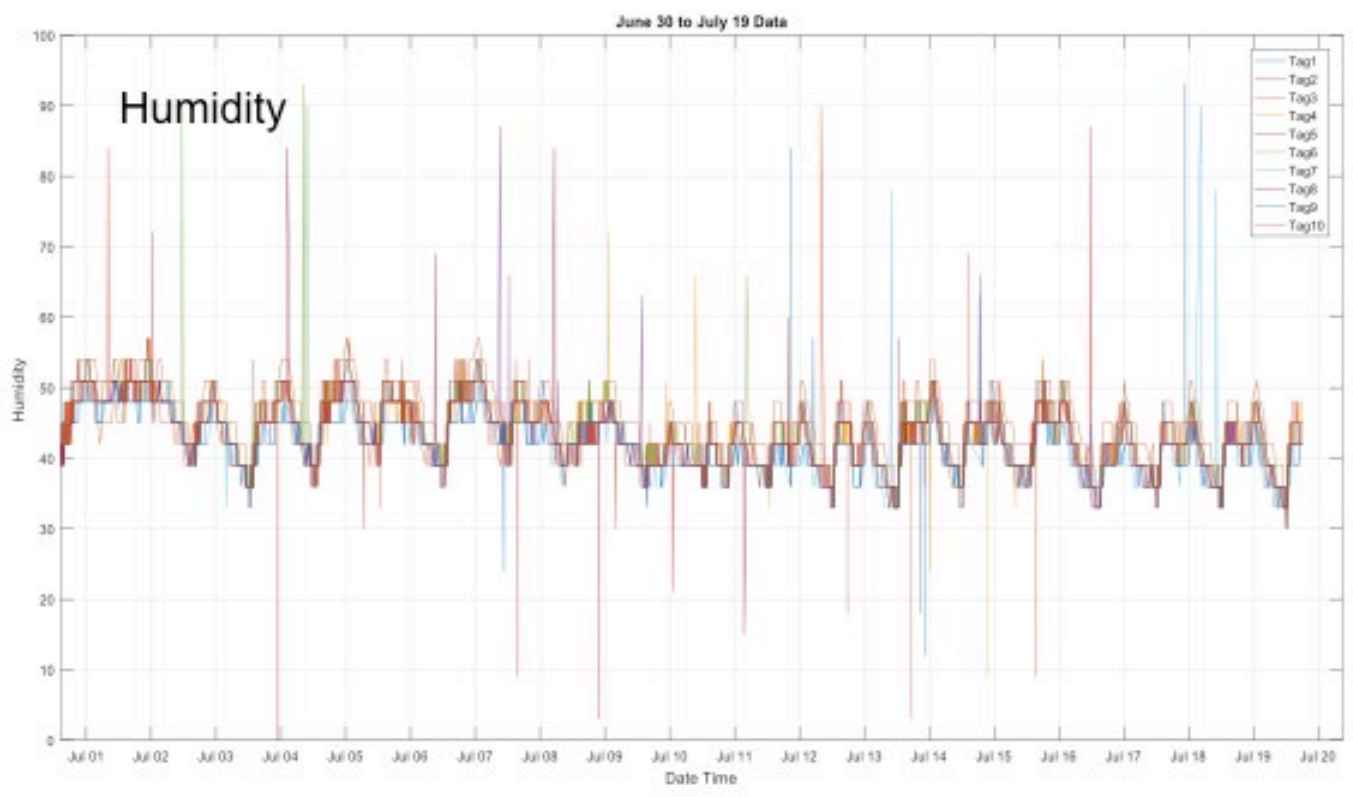

Figure 71 FRP\#1 sensor tags \#1 - \#10 humidity measurement June 30 to July 19. 
FRP\#1 July 01 to July 19 - Humidity Measurements

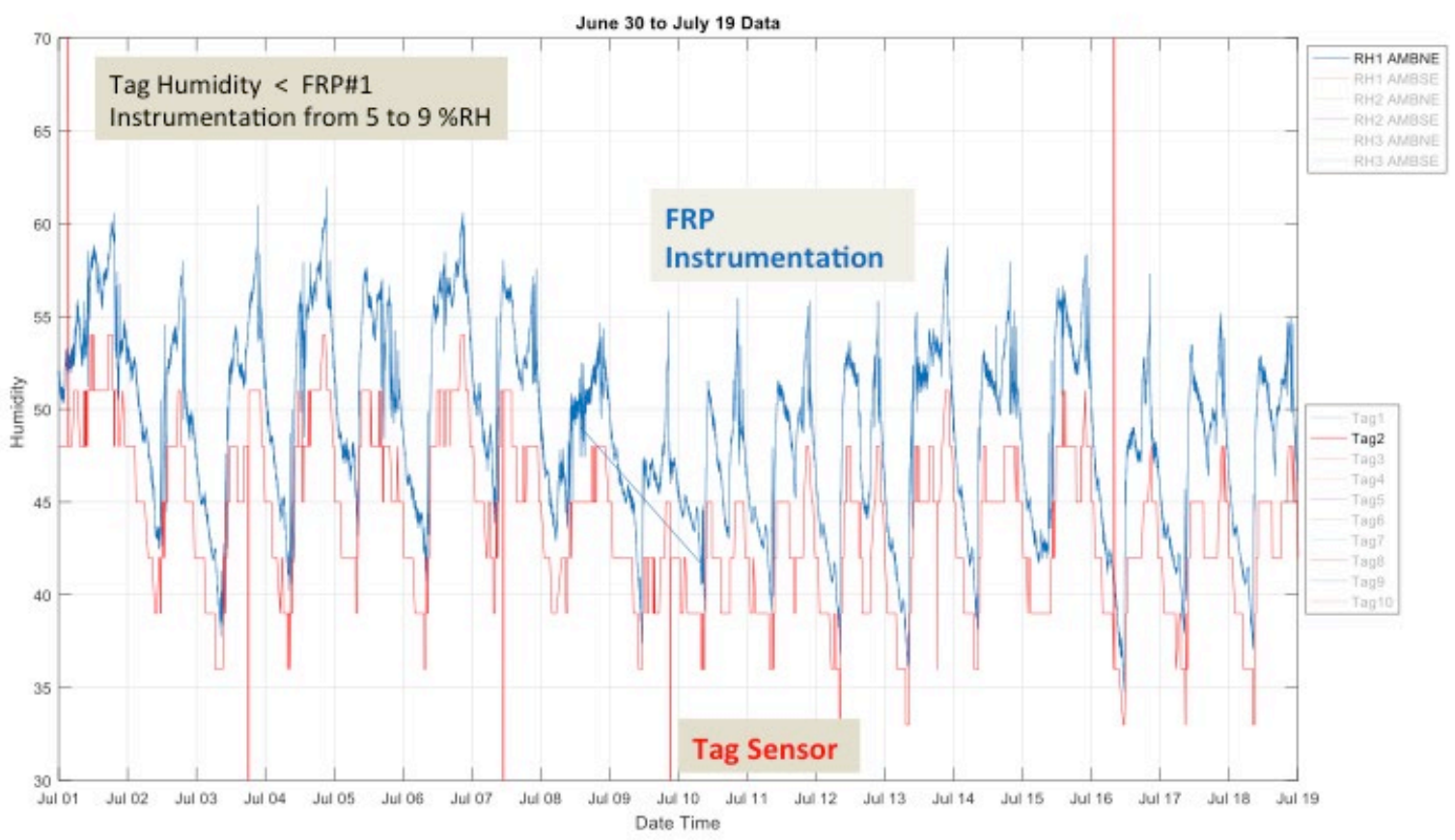

Figure 72 FRP\#1 sensor tag \#2 humidity compared with research instrumentation June 30 to July 19.

June 30 to July 19 , Tags \#1 - \#10

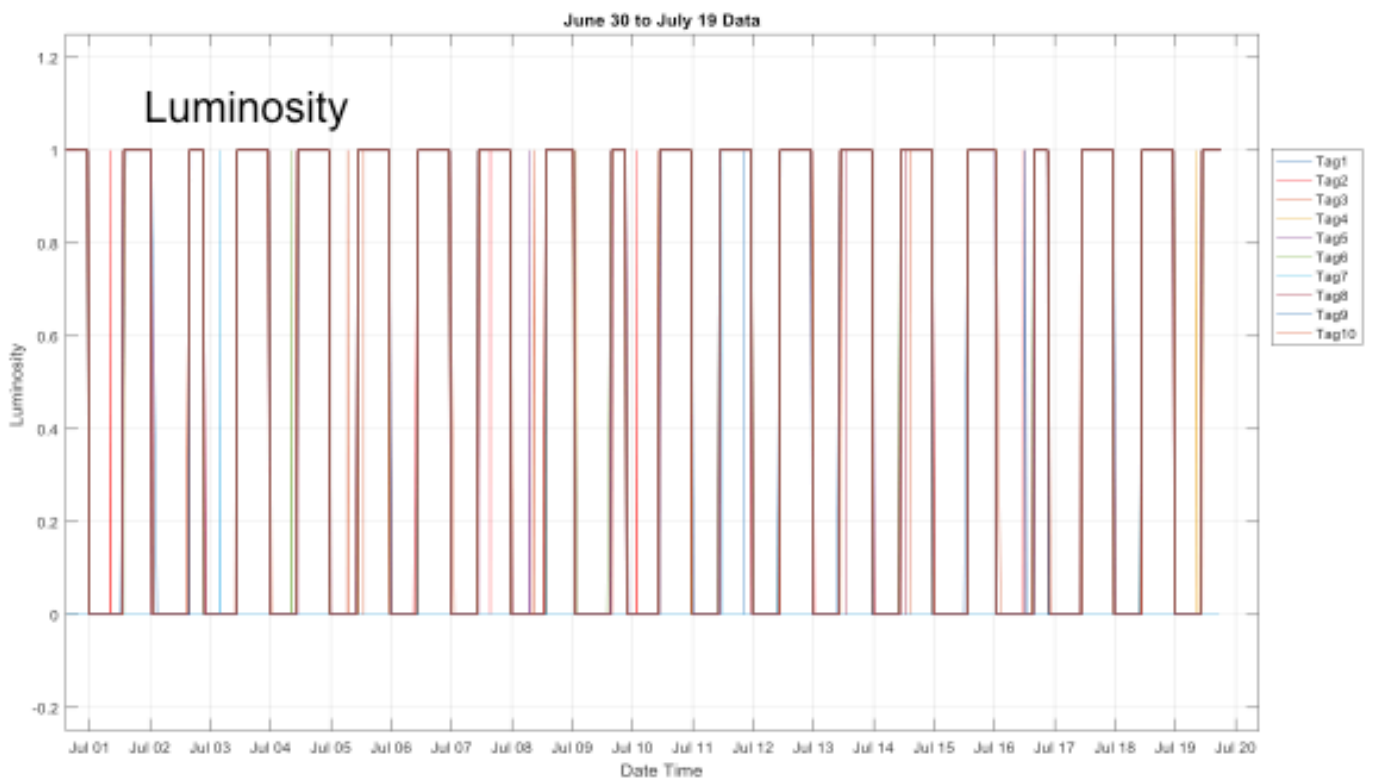

Figure 73 FRP\#1 sensor tags \#1 - \#10 luminosity (lights on/off) June 30 to July 19. 

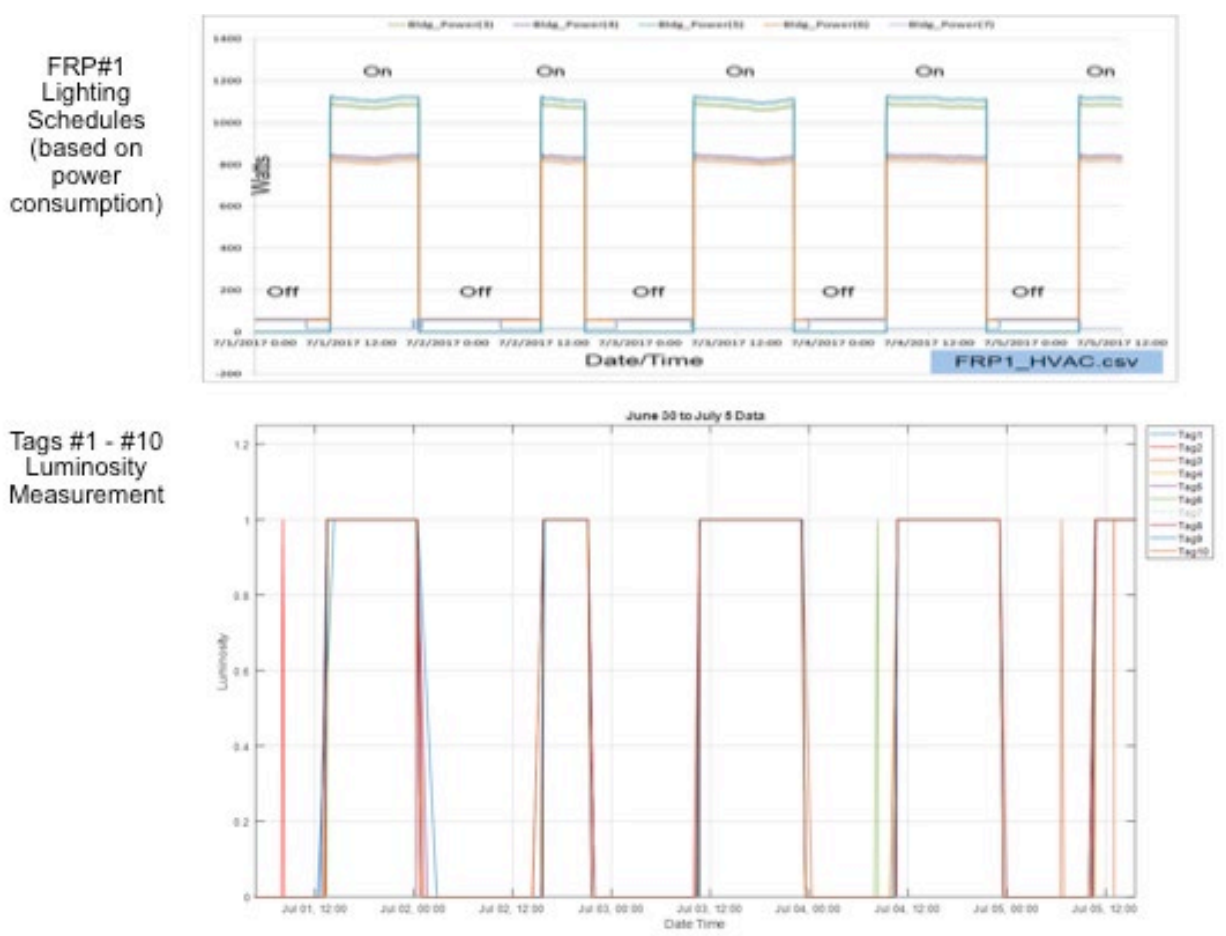

Figure 74 FRP\#1 sensor tags \#1 - \#10 luminosity (lights on/off) compared with FRP\#1 lighting schedules.

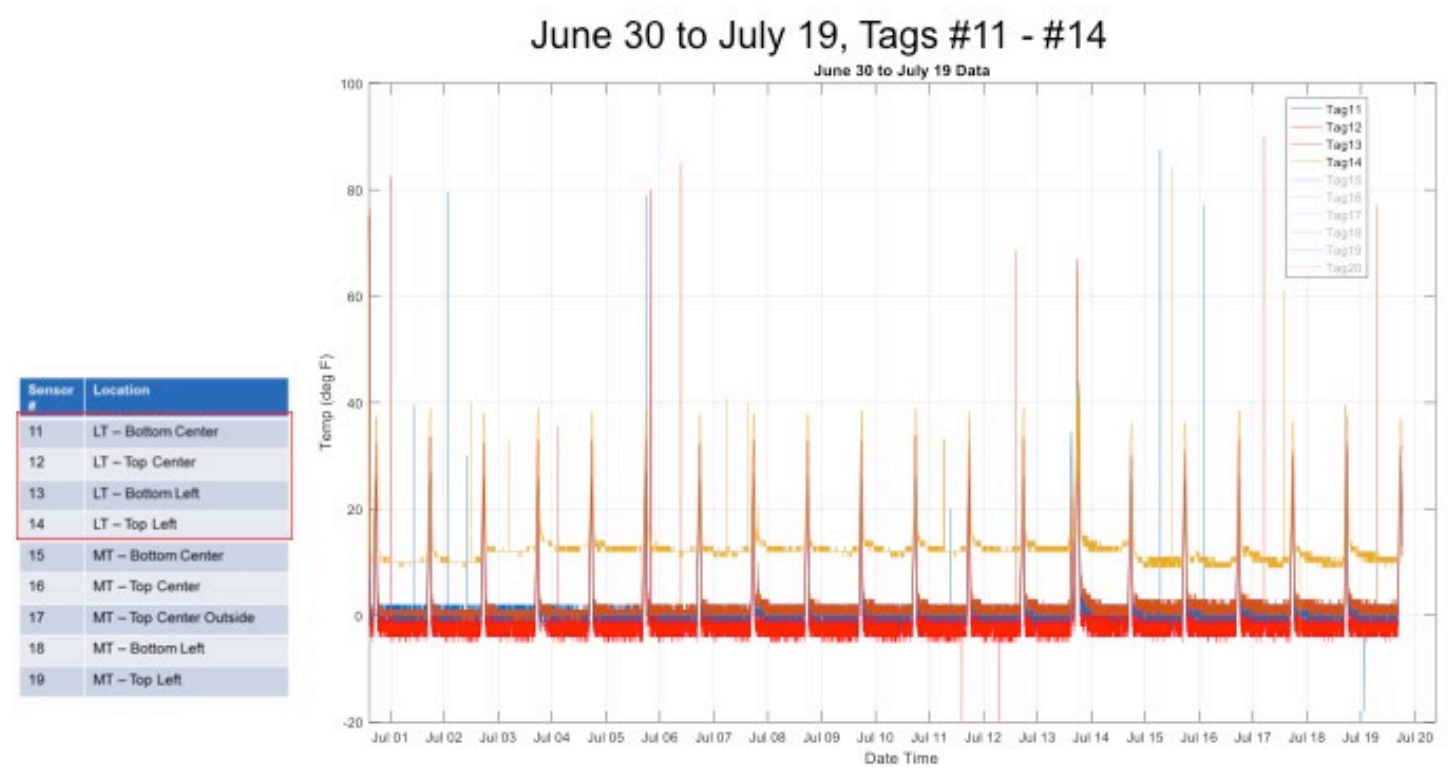

Figure 75 FRP\#1 sensor tags \#11 - \#14 temperature measurements June 30 to July 19 


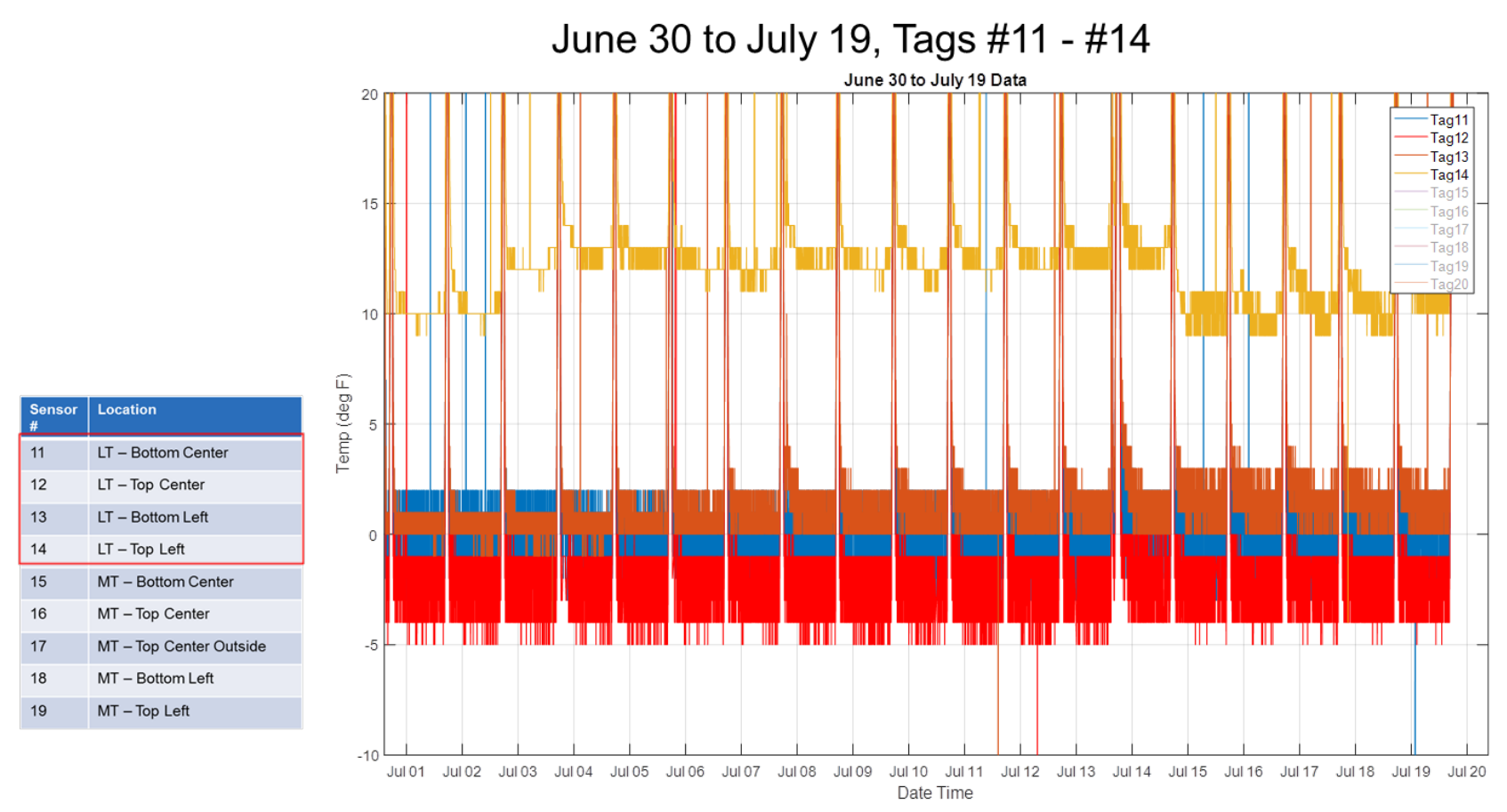

Figure 76 FRP\#1 sensor tags \#11 - \#14 temperature measurements June 30 to July 19

June 30 to July 19, Tags \#15-\#16, \#18-\#19

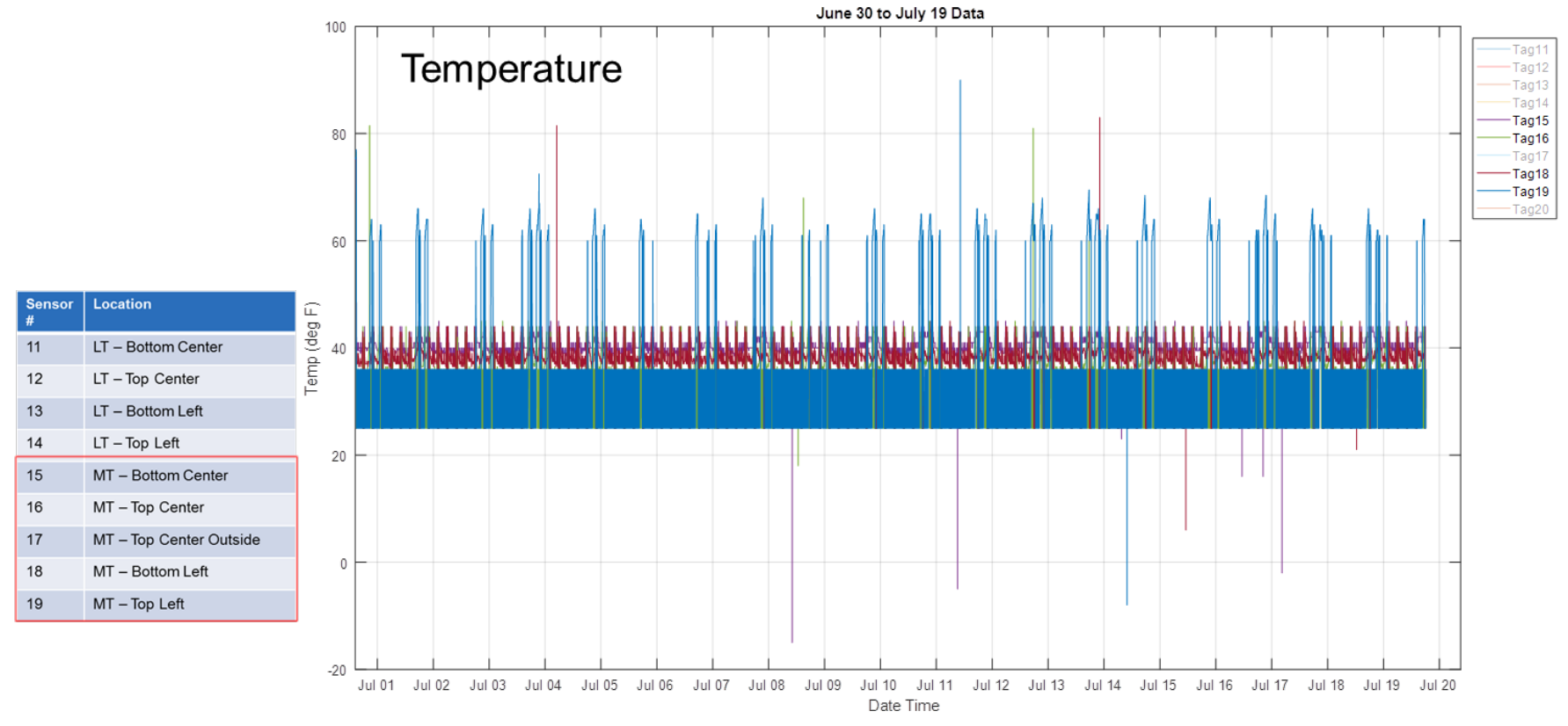

Figure 77 FRP\#1 sensor tags \#15 - \#16, \#18 - \#19 temperature measurements June 30 to July 19 


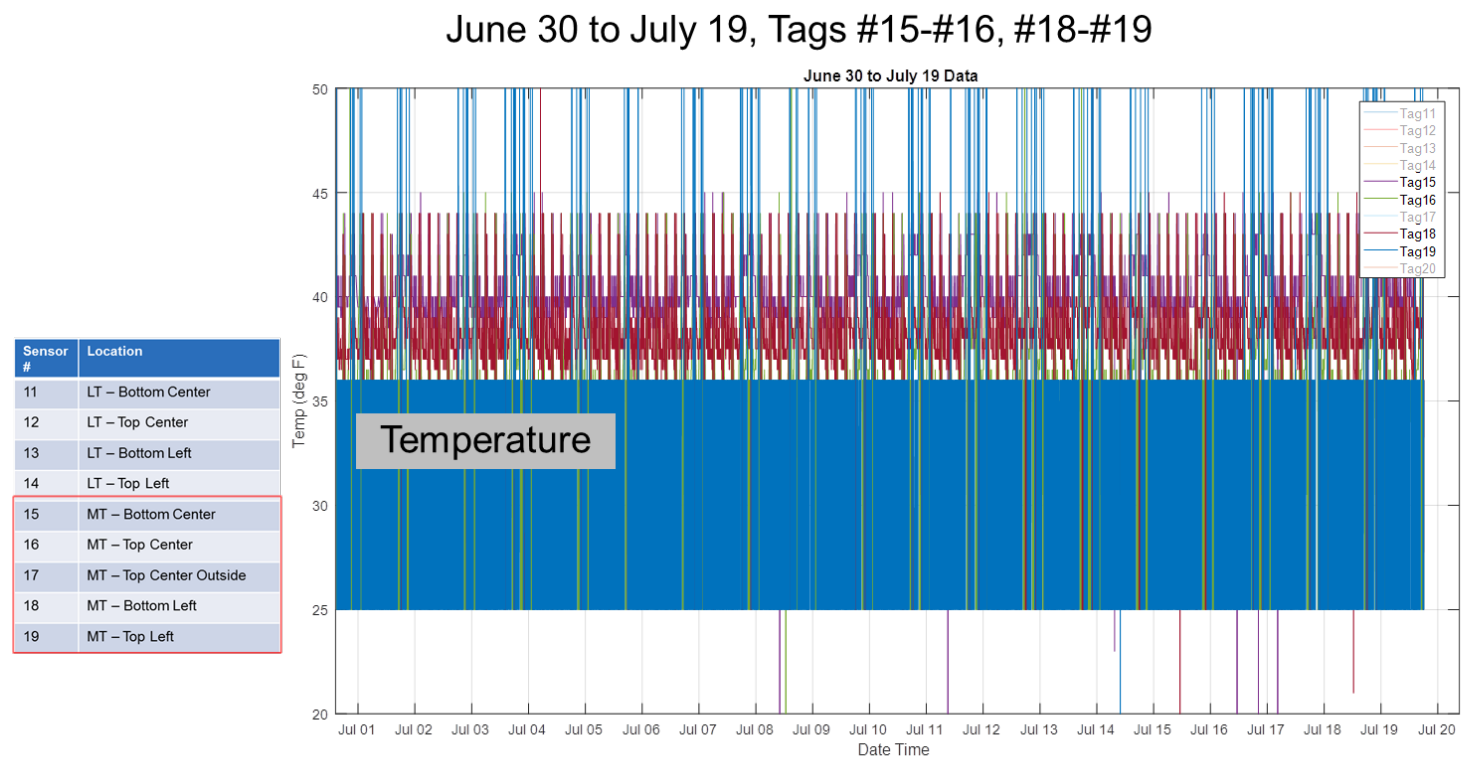

Figure 78 FRP\#1 sensor tags \#15 - \#16, \#18 - \#19 temperature measurements June 30 to July 19

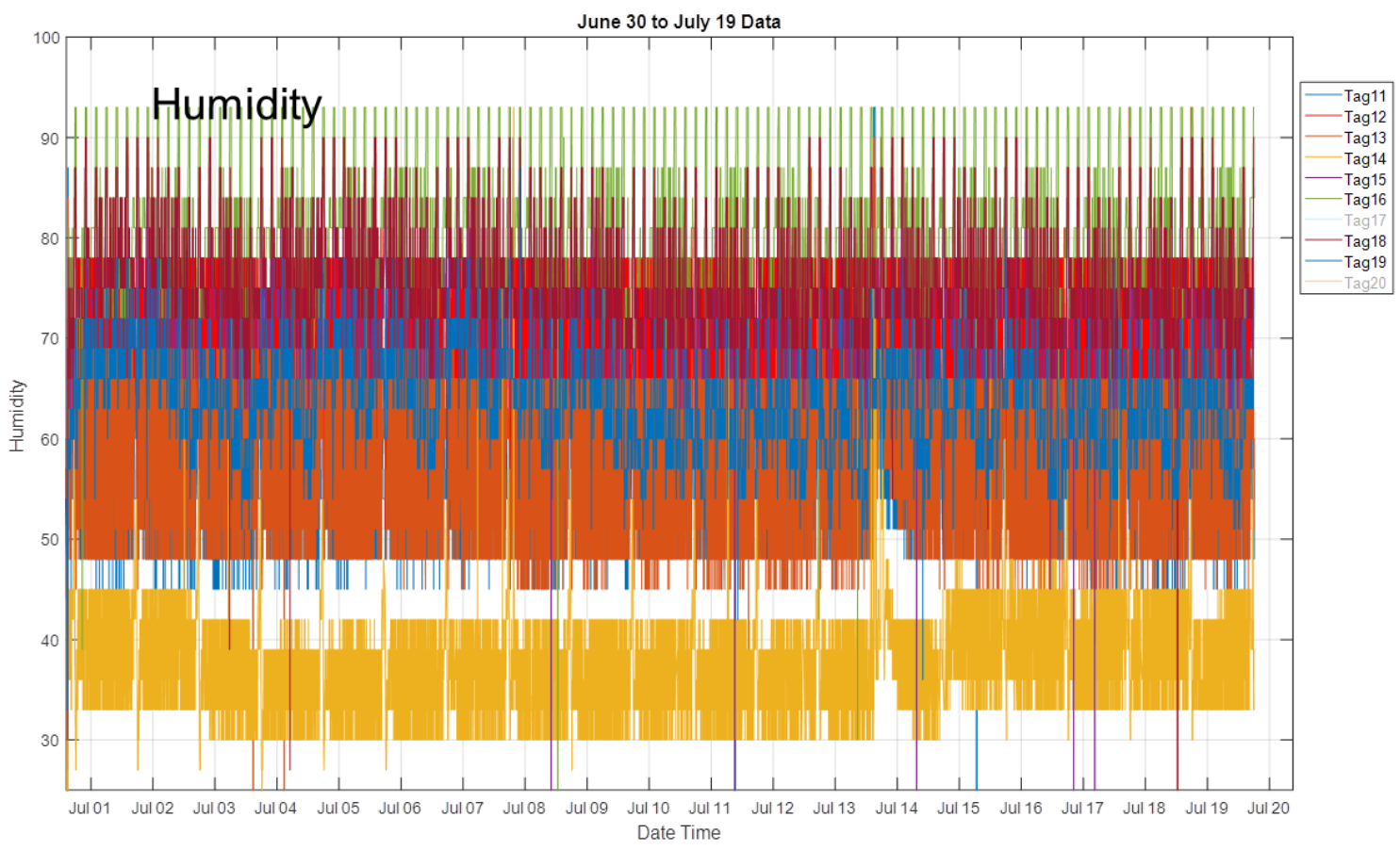

Figure 79 FRP\#1 sensor tags \#11 - \#14, 15 - \#16, \#18 - \#19 humidity measurements June 30 to July 19 in refrigerated case. 
June 30 to July 19, Tags \#11-\#16 \& \#18-\#19

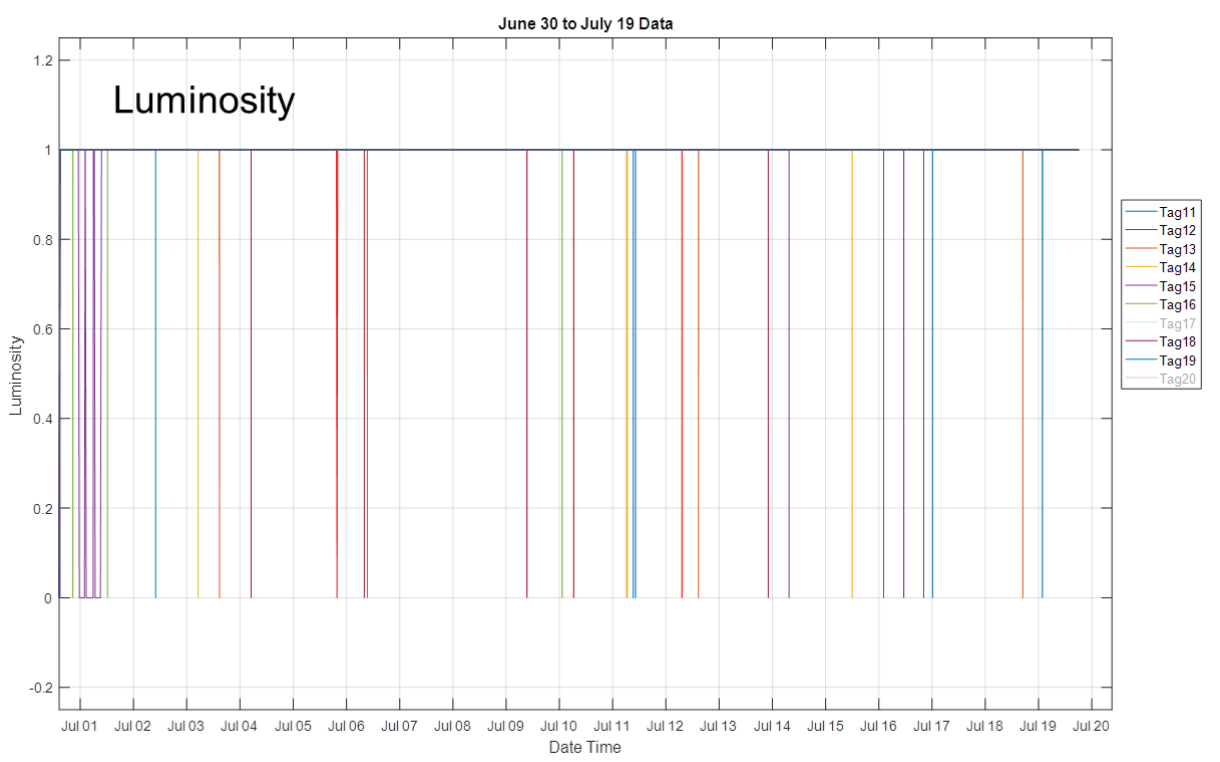

Figure 80 FRP\#1 sensor tags \#11 - \#14, 15 - \#16, \#18 - \#19 luminosity measurements June 30 to July 19 in refrigerated case.

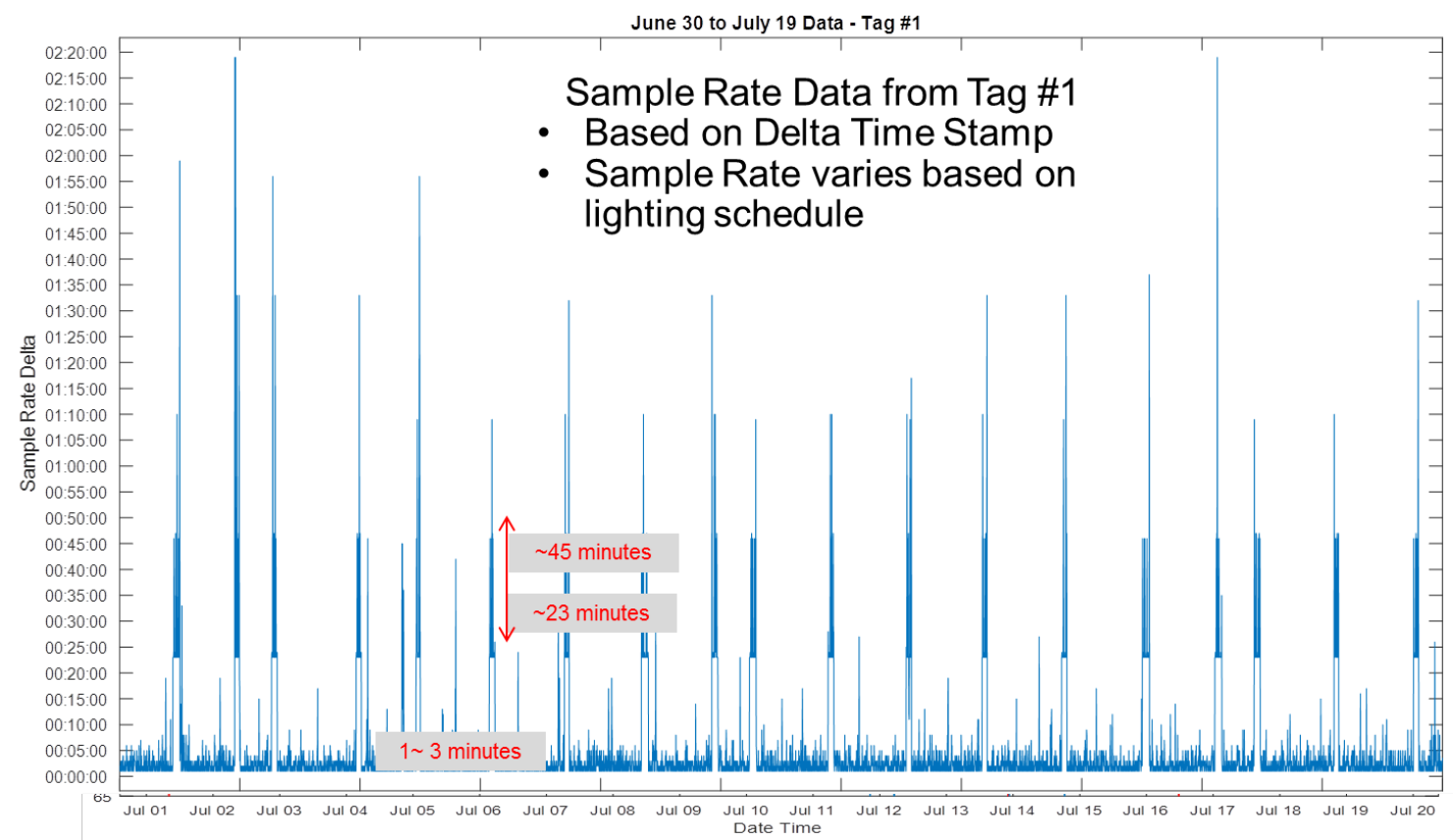

Figure 81 FRP\#1 sensor tag \#1 data sample rate from June 30 to July 19. 
June 30 to July 19, Tag \#11

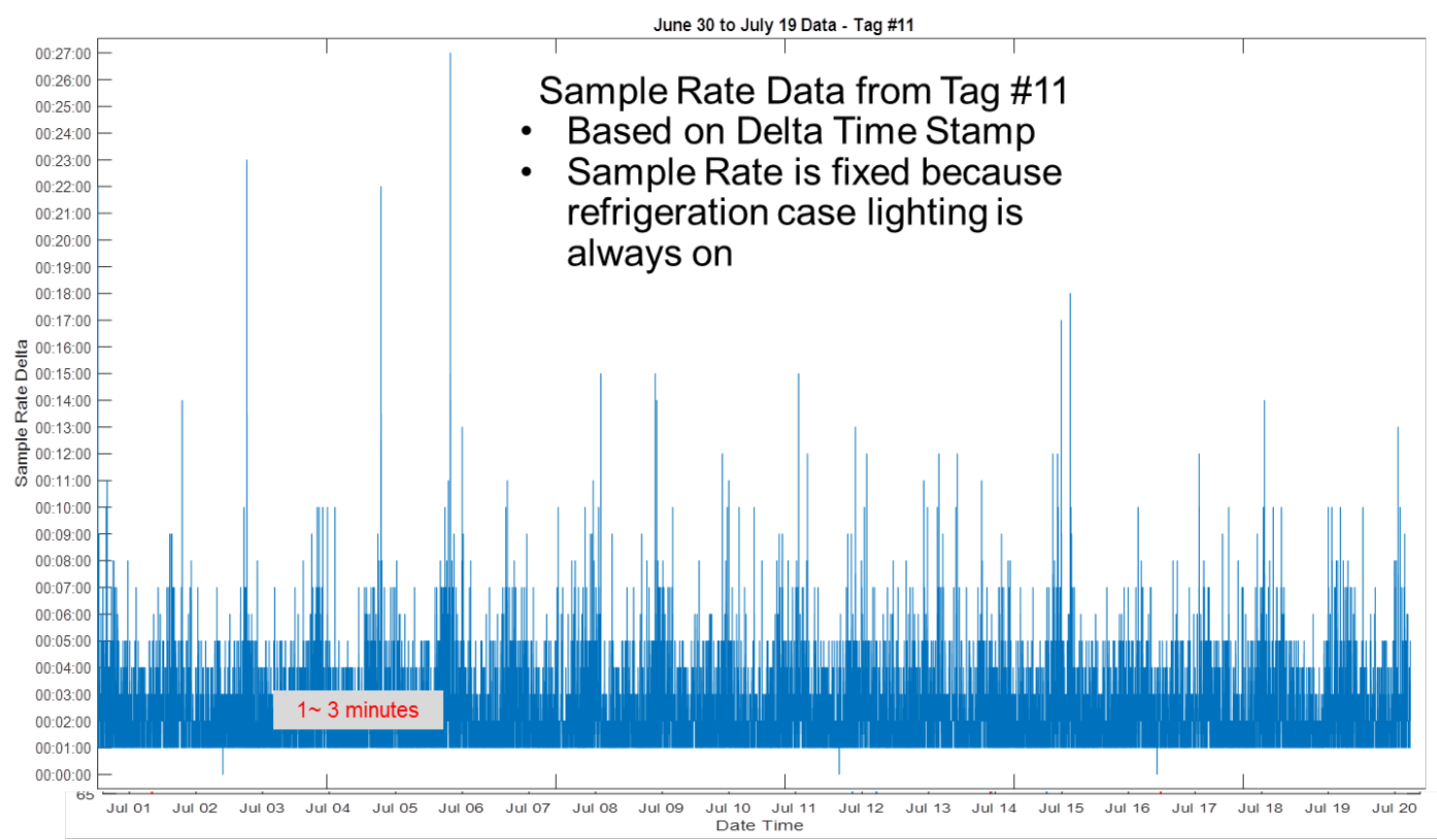

Figure 82 FRP\#1 sensor tag \#11 data sample rate from June 30 to July 19.

Data Points Synchronized to Show Data Sample Rate and Luminosity is Aligned
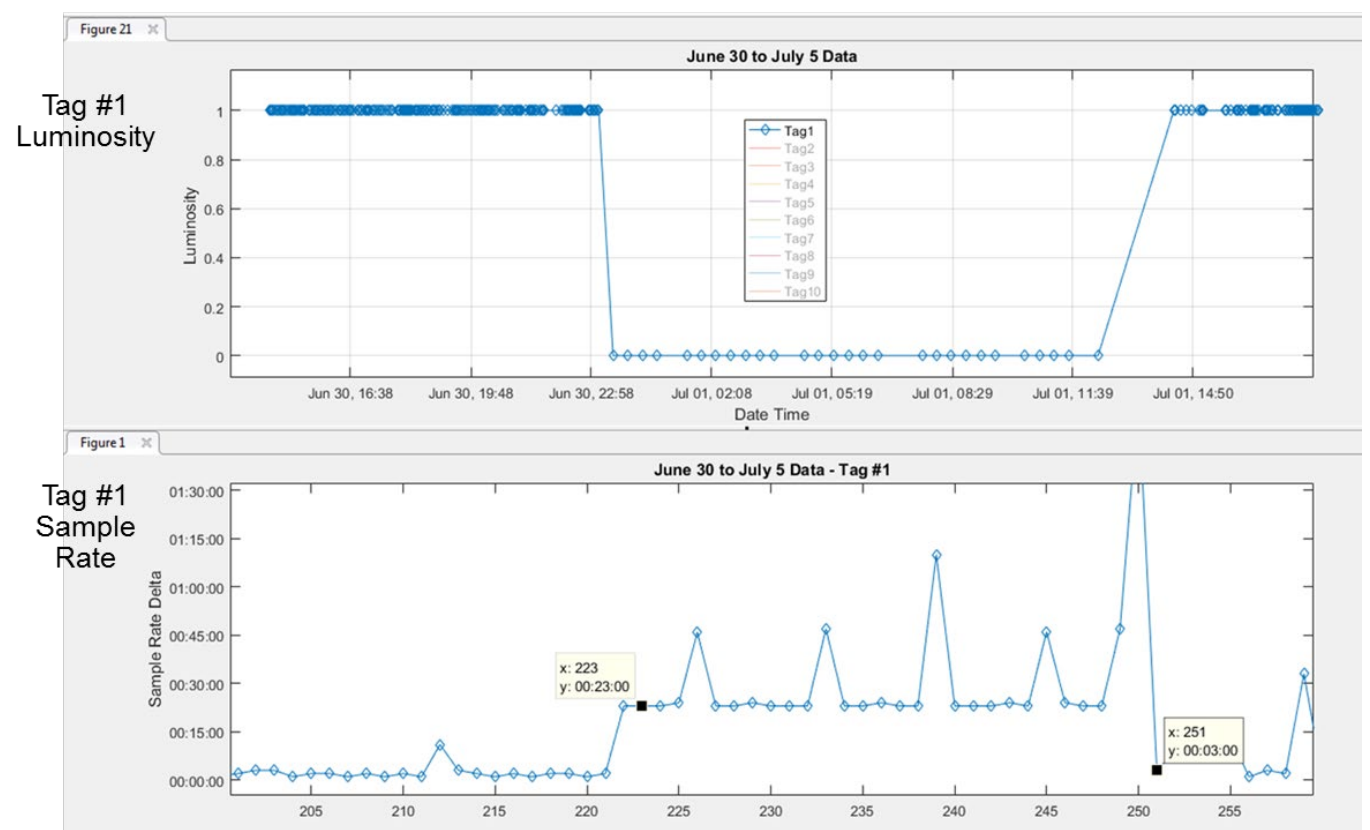

Figure 83 FRP\#1 sensor tag \#1 comparison of data sample rate versus luminosity measurement. 
Space Temperature Tags:

1. Min of 820 ( $\operatorname{Tag} \# 7)$

2. Max of 10864

3. Mean of 5545

Refrigeration Temperature Tags:

1. Min of 11317

2. Max of 12292

3. Mean of 1198

1. FRP\#1 lights are on a schedule.

2. Refrigeration Case Lights are always on

Data Transmit Rates

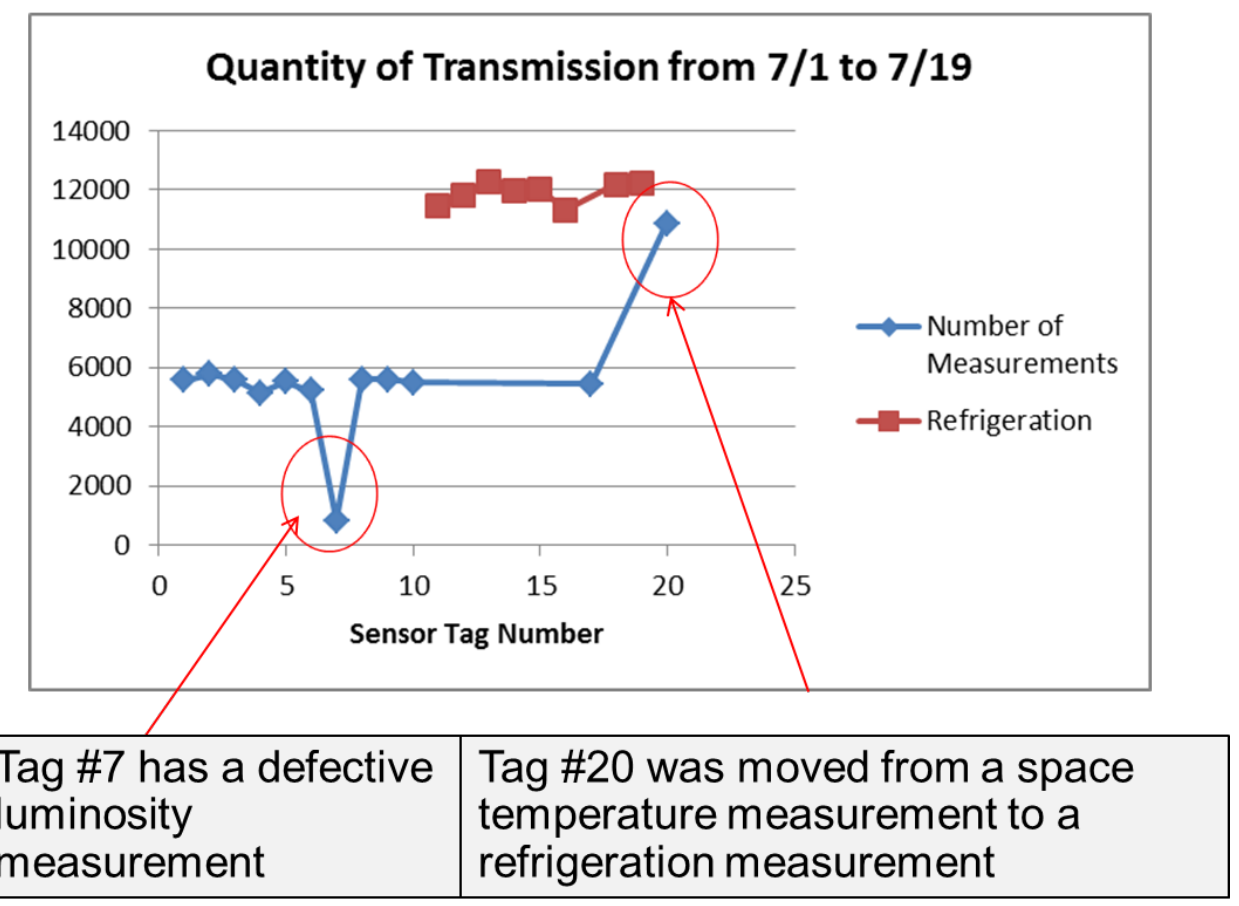

Figure 84 FRP\#1 sensor tag data transmission event analysis.

\section{FRP\#2 TEST DEPLOYMENT}

Nineteen sensors were deployed in FRP\#2 in July on the first and second floors. Eight sensor tags were located on the first floor (Figure 85) and 11 sensor tags were located on the second floor (Figure 86). The receiver was located on the second floor in Room 205 (noted as RX in Figure 86). Sensor tags \#1 - \#6 on the first floor were in conditioned spaces, and sensor tags \#7-\#8 were in the unconditioned space of the stairwell. On the second floor, sensor tags $\# 9$ and \#10 were in the unconditioned stairwell, and sensor tags $\# 11-\# 17, \# 19$, and \#20 were in the conditioned spaces. Figure 87 -Figure 91 illustrate the installation locations of some of the sensors on the first floor. The installation of the sensors and the receiver required about 1 hour for installation and documentation. 


\begin{tabular}{|l|l|}
\hline $\begin{array}{l}\text { Sensor } \\
\#\end{array}$ & Location \\
\hline 1 & Room 102 \\
\hline 2 & Room 106 \\
\hline 3 & Room 105 \\
\hline 4 & Room 105 \\
\hline 5 & Room 104 \\
\hline 6 & Room 104 \\
\hline 7 & Room 101 \\
\hline 8 & Room 101 \\
\hline
\end{tabular}

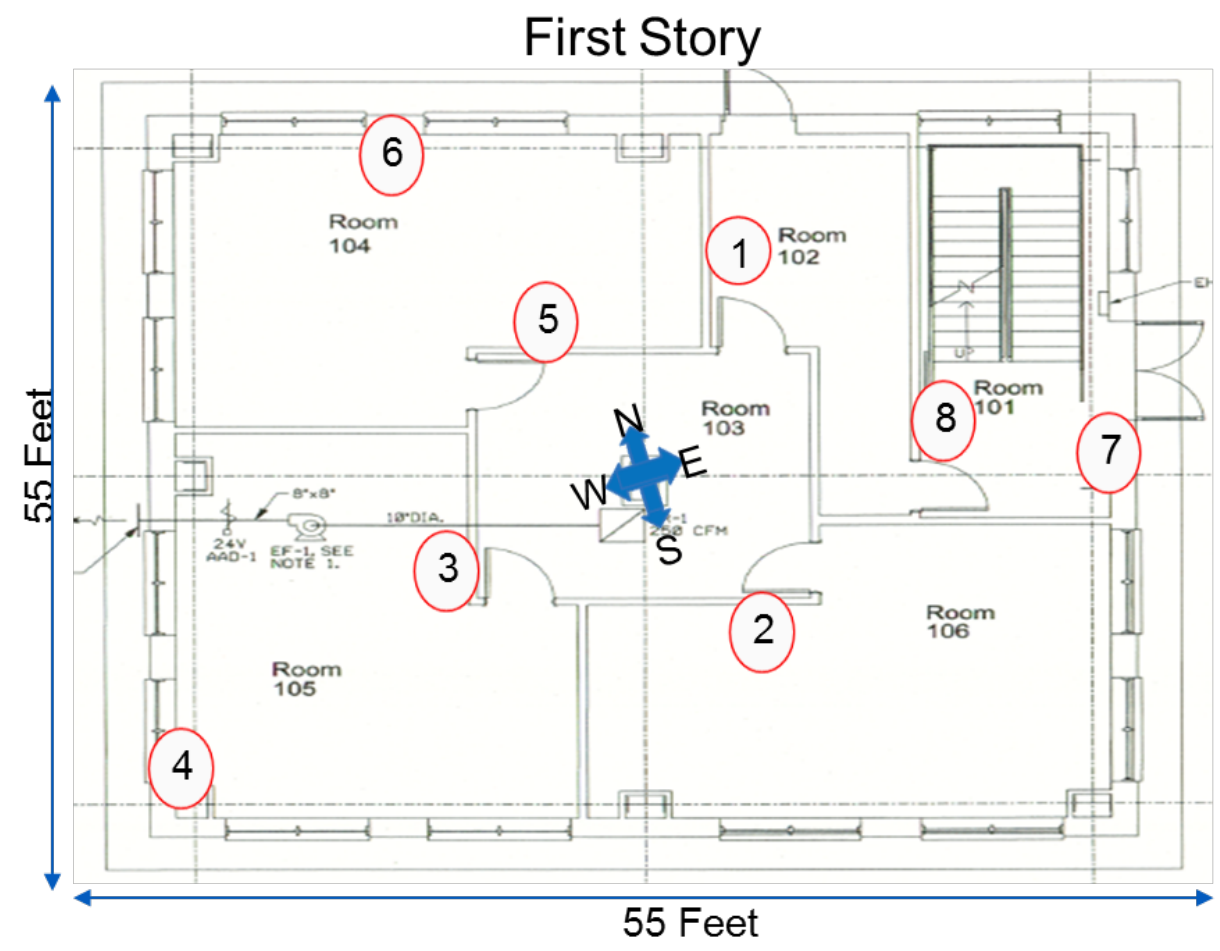

Figure 85 FRP\#2 space temperature measurement sensor locations on first floor.

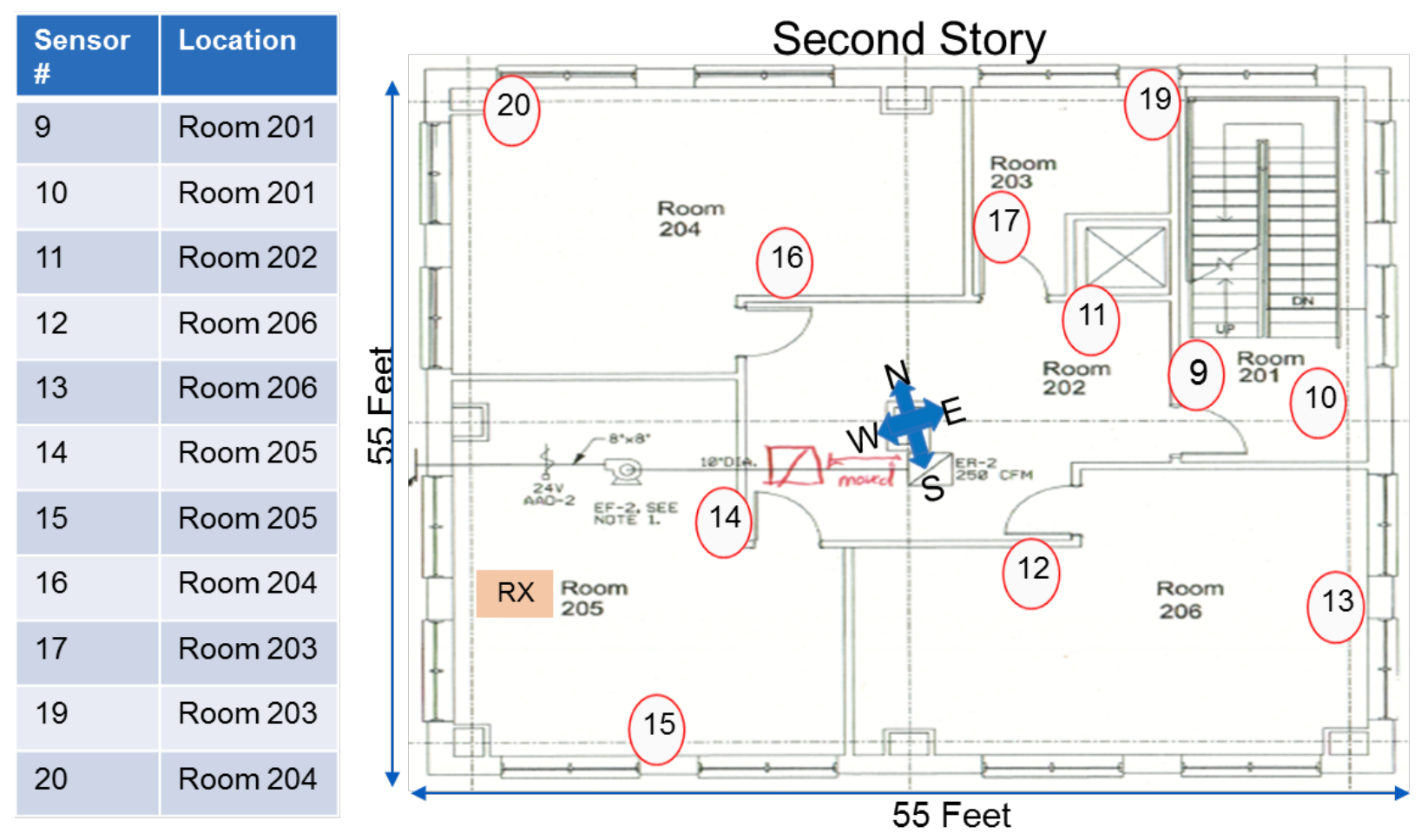

Figure 86 FRP\#2 space temperature measurement sensor locations on second floor. 


\section{Sensor \#1}
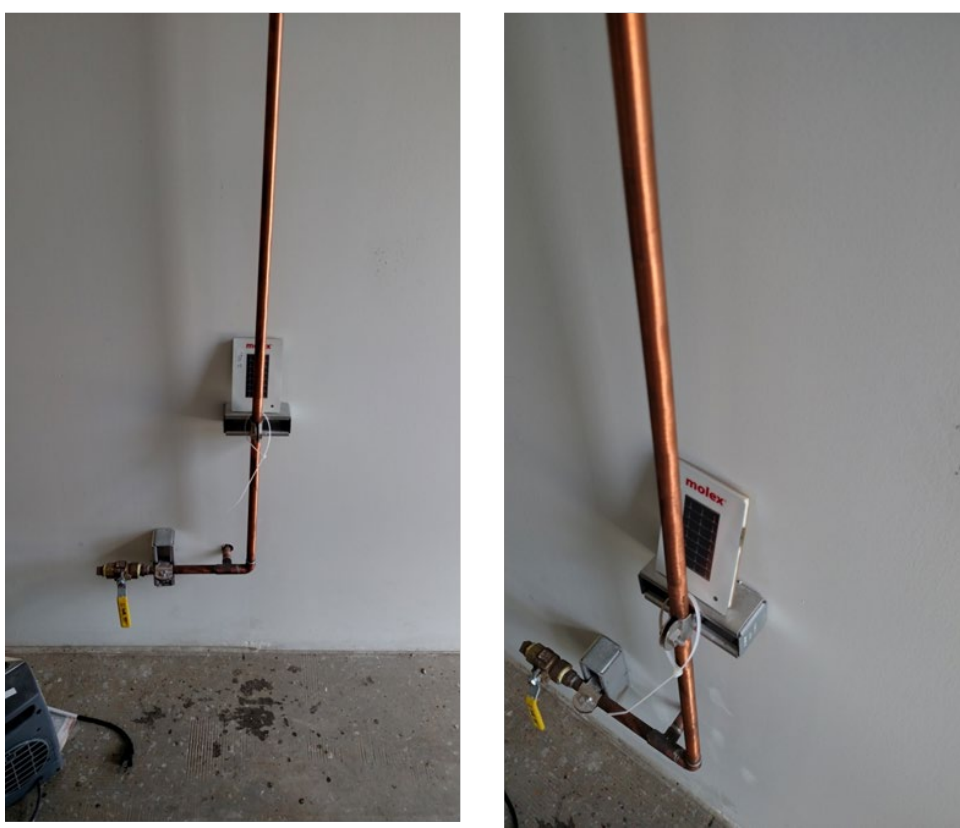

Figure 87 FRP\#2 sensor tag \#1 location (first floor Room 102).

\section{Sensor \#2}
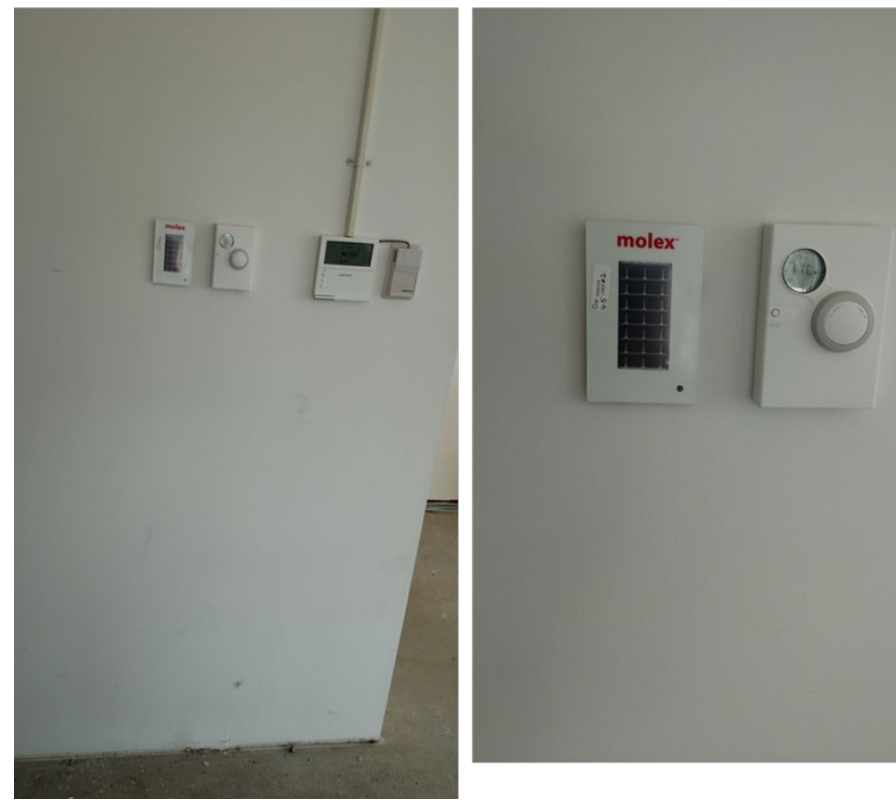

Figure 88 FRP\#2 sensor tag \#2 location (first floor Room 106). 


\section{Sensor \#3}
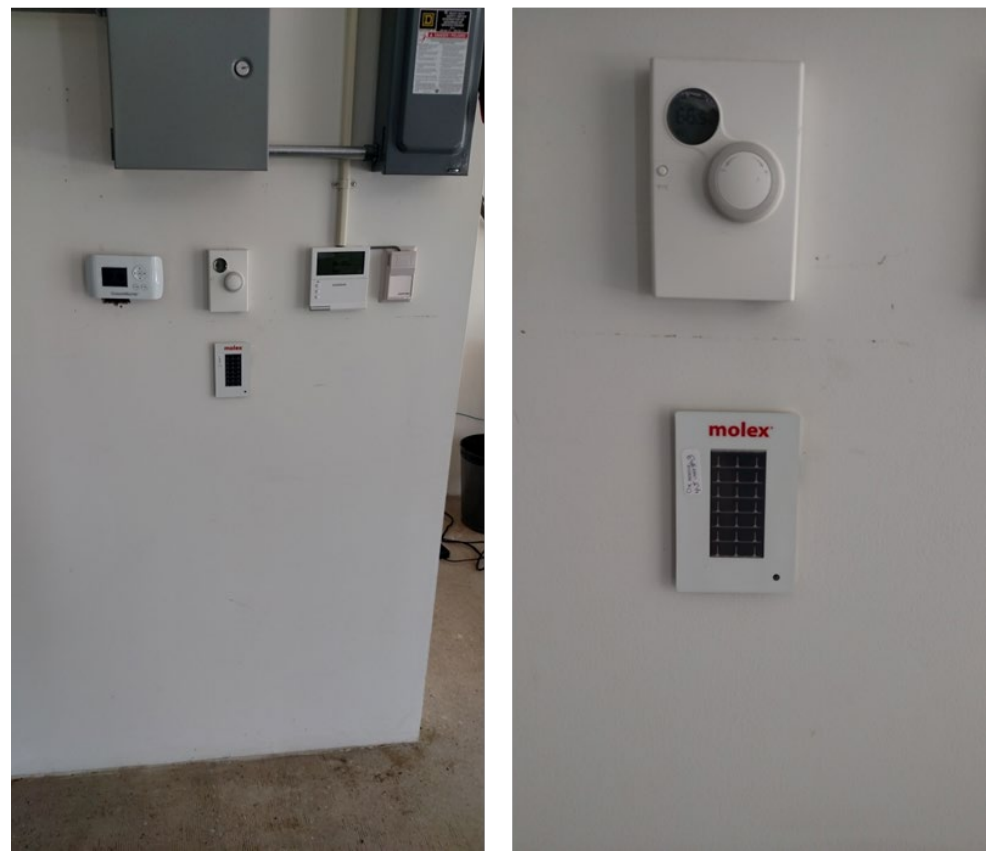

Figure 89 FRP\#2 sensor tag \#3 location (first floor Room 105).

\section{Sensor \#5}
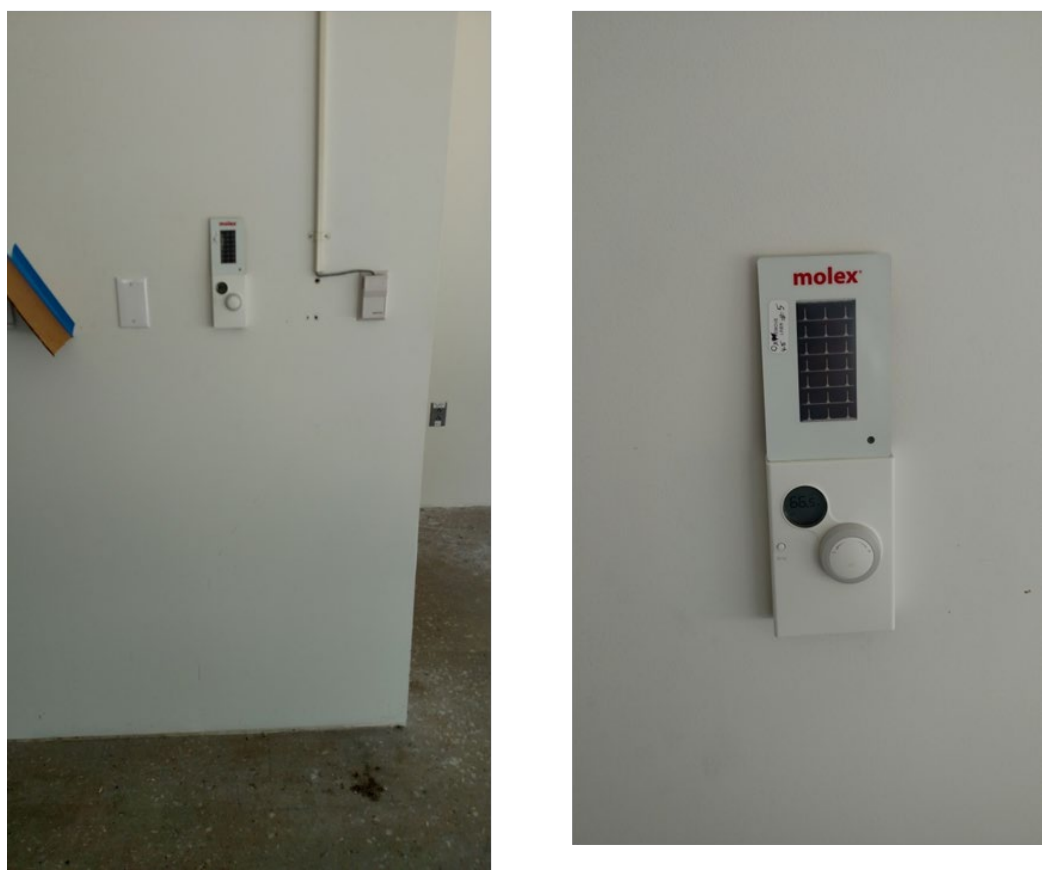

Figure 90 FRP\#2 sensor tag \#5 location (first floor Room 104). 


\section{Sensor \#6}

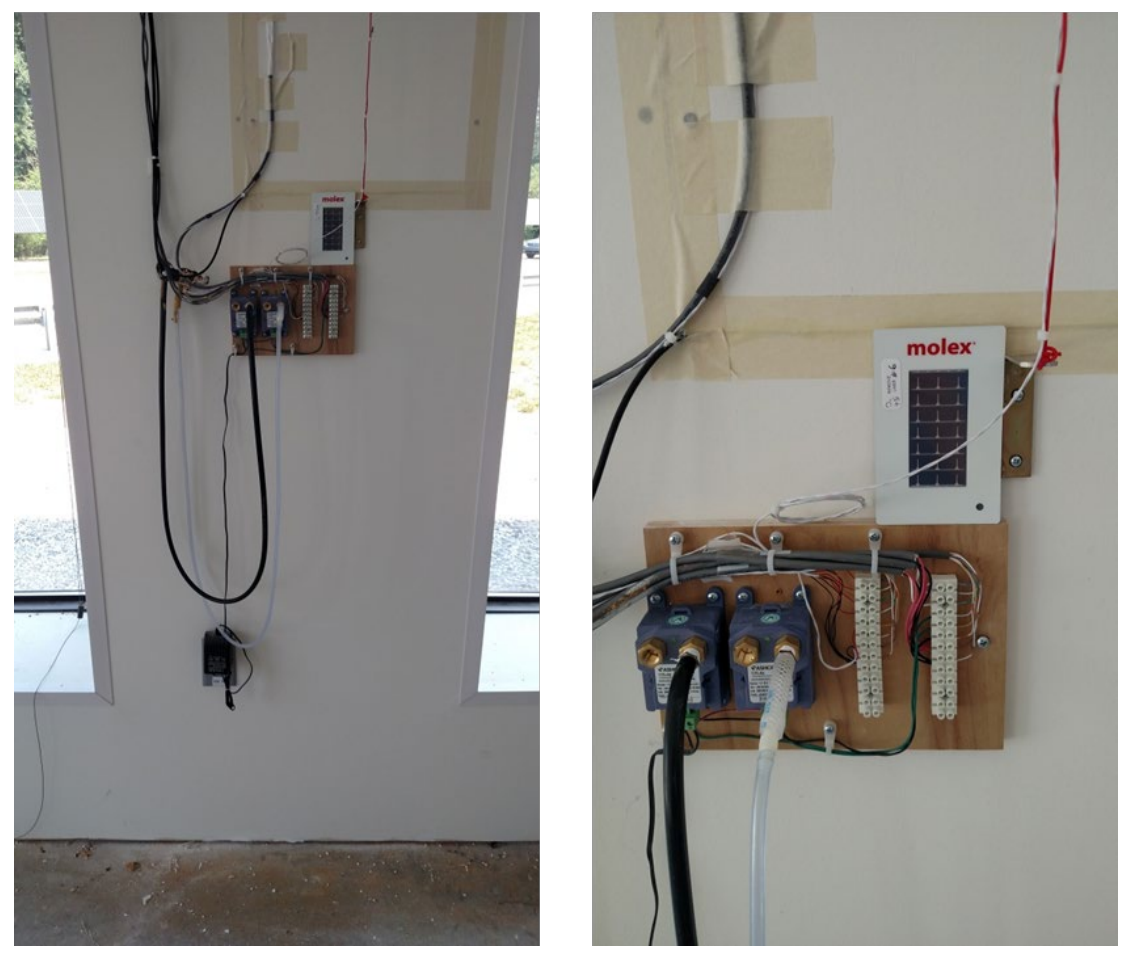

Figure 91 FRP\#2 sensor tag \#6 location (first floor Room 104).

\section{FRP\#2 TEST DEPLOYMENT DATA ANALYSIS}

Data were collected from the FRP\#2 testing for analysis to quantify the sensor test performance. The analysis in this report contains data from July 19-27. Figure 92 illustrates space temperature measurements for sensors \#1 through \#8 on the first floor. A selection of sensor tags \#1 -\#3, \#5, and \#6 space temperature measurements, which are all located in interior space locations that are well cooled, is shown in Figure 93. The first floor sensor tags \#4, \#7, and \#8 space temperature measurements are shown in Figure 94. Sensor tag \#4 is in a west-facing window and receives afternoon sun. Sensor tags \#7 and \#8 are in the first floor stairwell unconditioned space. The second floor tags $\# 11-\# 17, \# 19$, and \#20 are in well-cooled interior locations; their space temperature measurements are shown in Figure 95. The second floor tags \#9 and \#10 are in the unconditioned stairwell; their space temperature measurements are shown in Figure 96.

The first floor sensor tags \#1 - \#8 humidity measurements are shown in Figure 97. The coolertemperature locations (sensor tags \#1 - \#6) have higher relative humidity measurements versus the warmer stairwell locations (sensor tags \#7 and \#8). The second floor sensor tags \#9-\#17, \#19, and \#20 humidity measurements are shown in Figure 98. Similar to the first floor, the interior cooler locations (sensor tags \#11 - \#17, \#19, and \#20) have higher relative humidity values than the warmer stairwell locations (sensor tags \#9 and \#10).

Figure 99 illustrates the luminosity (lights on/off) measurements for the first floor sensor tags \#1 - \#8. All of these locations have windows and scheduled interior lighting, which create periods of lights-on and lights-off conditions. The data show that the different locations have differing periods of lighting. The luminosity (lights on/off) measurements for the second floor sensor tags $\# 9$ - \#17, \#19, and \#20 are 
shown in Figure 100. Sensor tag location \#11 is in Room 202 (center of the building), which does not have windows and has very low light levels. This is shown in the sensor tag \#11 luminosity data in Fig. 47.

The sensors are configured to operate with different sample and transmit rates, based on the lighting levels, to conserve battery power at times when the solar cell is not charging the battery. If the lights are on, the sensor transmits every 90 seconds and if the lights are not on, the sensor transmits every 22 minutes. Figure 102 and Figure 103 illustrate that sensor tags \#1 and \#2 vary the transmit rate during lights-on and lights-off periods. Sensor tag \#11 was noted to be in a low-light location, and the reduced

data transmit rates of 25 minutes is shown in Figure 104. This relationship is illustrated in more detail in Figure 105 with a comparison of the sensor tag \#11 data transmission rate and the luminosity (lights on/off) measurement. Another measure of data transmission is the total quantity of data transmission events. Figure 106 illustrates the data transmission quantities for the different sensor locations. Note that the two low-light locations (sensor tags \#1 and \#11) have reduced data transmit events.

\section{CONCLUSIONS AND NEXT STEPS}

Thirty-nine sensor tags were deployed in FRP\#1 and FRP\#2 in conditioned spaces, unconditioned spaces, a low-temperature refrigeration case, and a medium-temperature refrigeration case. The sensors were exposed to temperatures ranging from $-5^{\circ} \mathrm{F}$ (low-temperature refrigeration case in FRP\#1) to $90^{\circ} \mathrm{F}$ (unconditioned stairwell in FRP\#2). Sensor tags were compared with research thermocouples and were found to be within $0.5^{\circ} \mathrm{F}$. Sensor tag humidity measurements were compared with research instrumentation and were found to be consistently 5-9\% RH lower than the research instrumentation. The sensor tag luminosity (lights on/off) measurement was found to correlate with the building lighting schedule. The receiver system successfully collected transmission data and provided those data to the collection computing device. The testing on FRP\#1 and FRP\#2 will continue to provide more experience and data for additional comparisons with building instrumentation, additional wireless data transmission study, and deployment maturation. 
Tags \#1 - \#8, Temperature Measurement

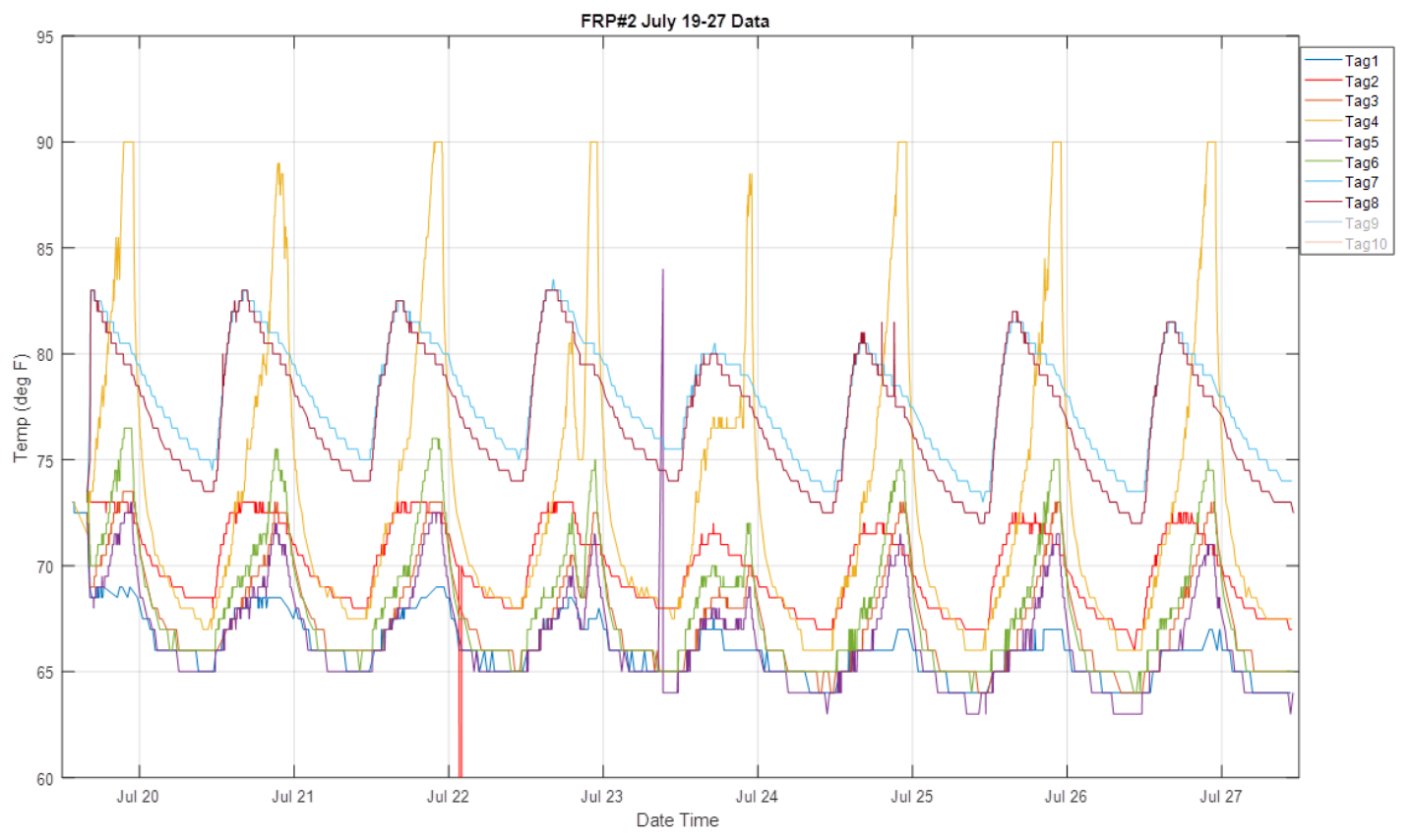

Figure 92 FRP\#2 sensor tags \#1-\#8 (first floor) temperature measurements July 19-27.

Tags \#1-\#3, \#5-\#6, Temperature Measurement

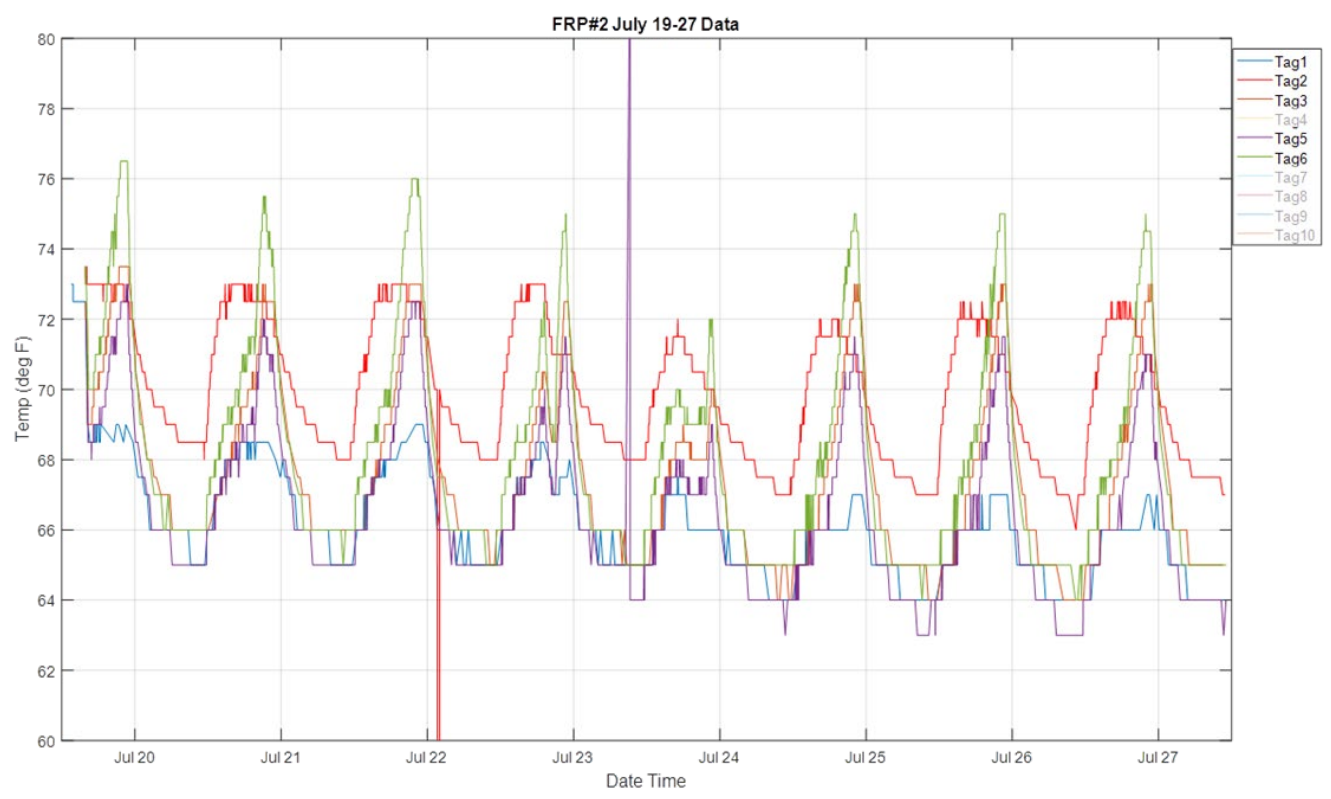

Figure 93 FRP\#2 sensor tags \#1, \#3, \#5, \#6 (first floor) temperature measurements July 19-27. 
Tags \#4, \#7-\#8 Temperature Measurement

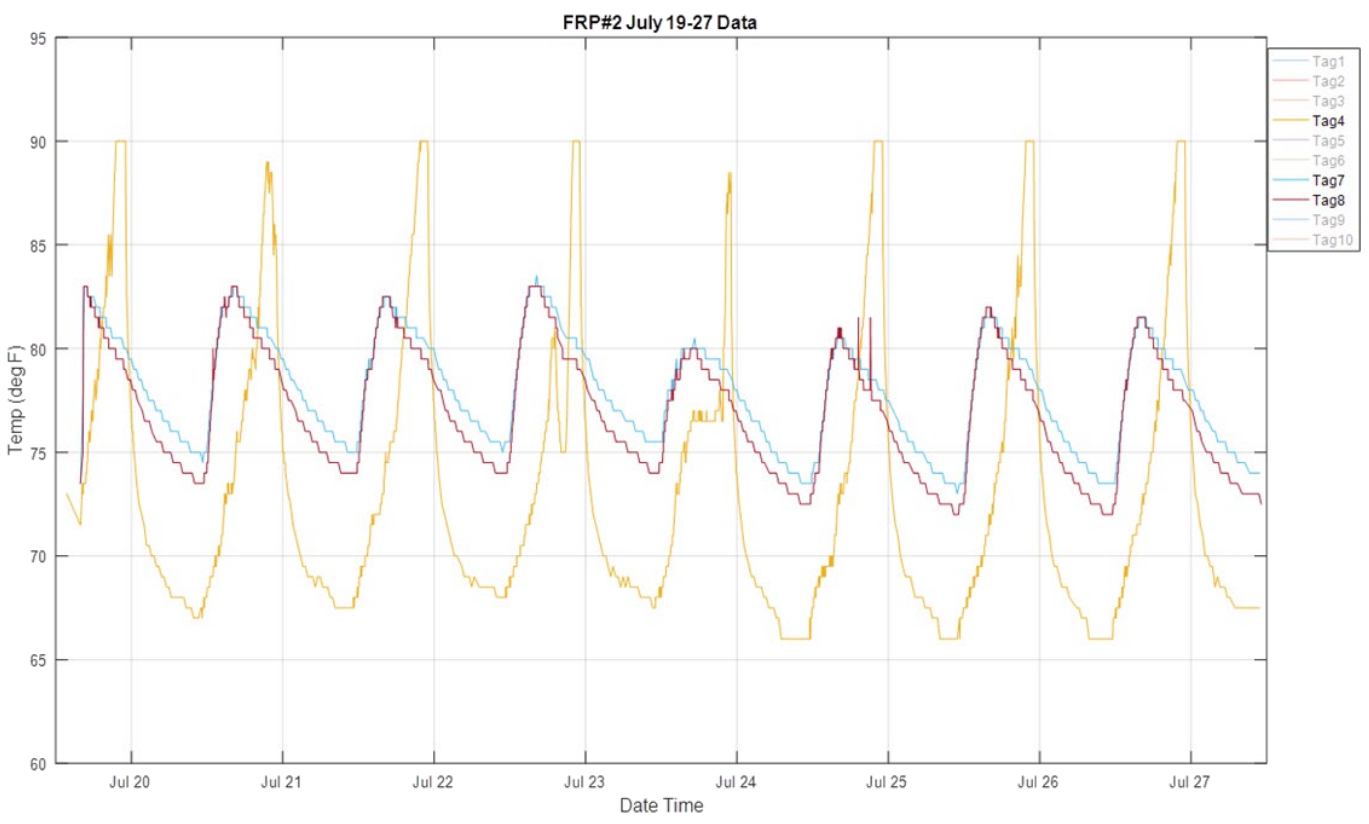

Figure 94 FRP\#2 sensor tags \#4, \#7, \#8 (first floor) temperature measurements July 19-27.

\section{Tags \#11-\#17, \#19-\#20 Temperature Measurement}

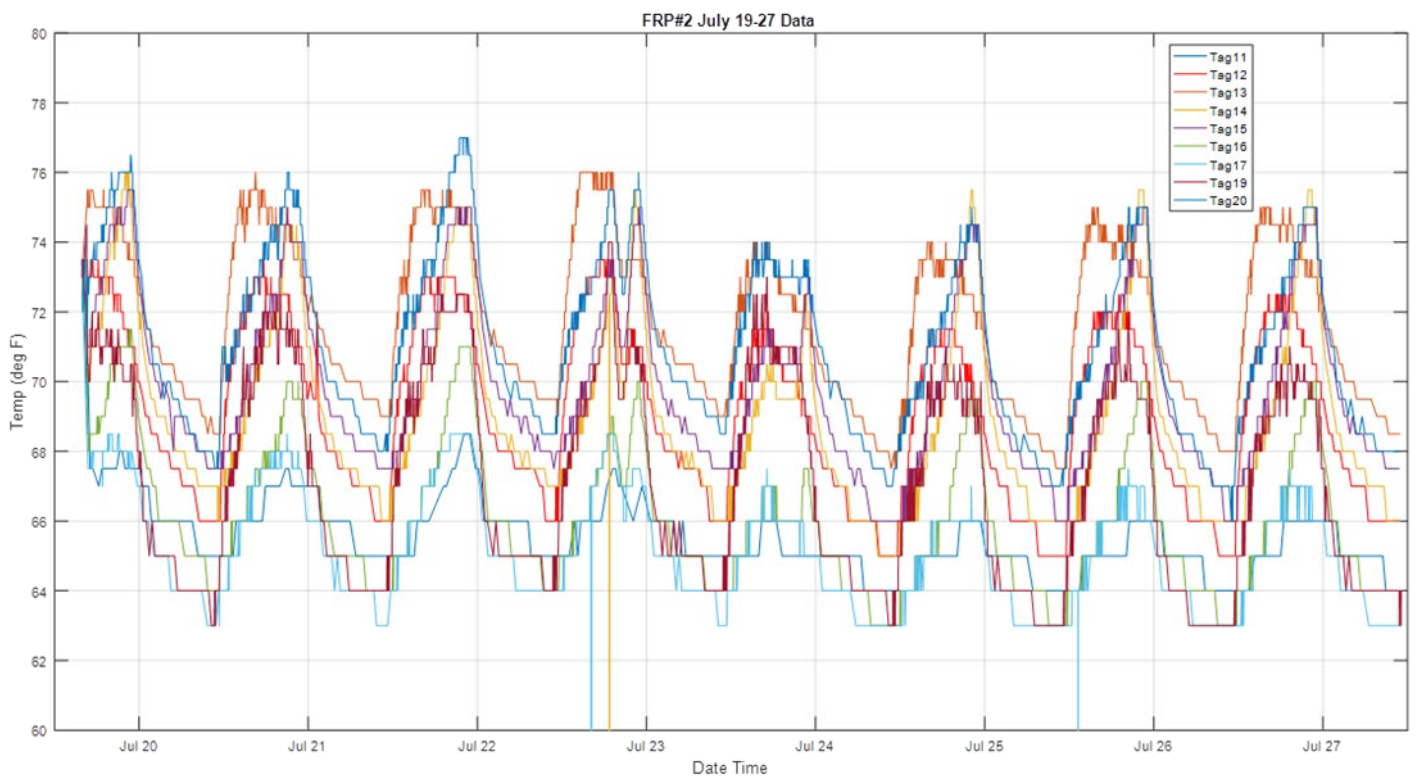

Figure 95 FRP\#2 sensor tags \#11-\#17, \#19, and \#20 (second floor) temperature measurements July 19-27. 


\section{Tags \#9-\#10 Temperature Measurements}

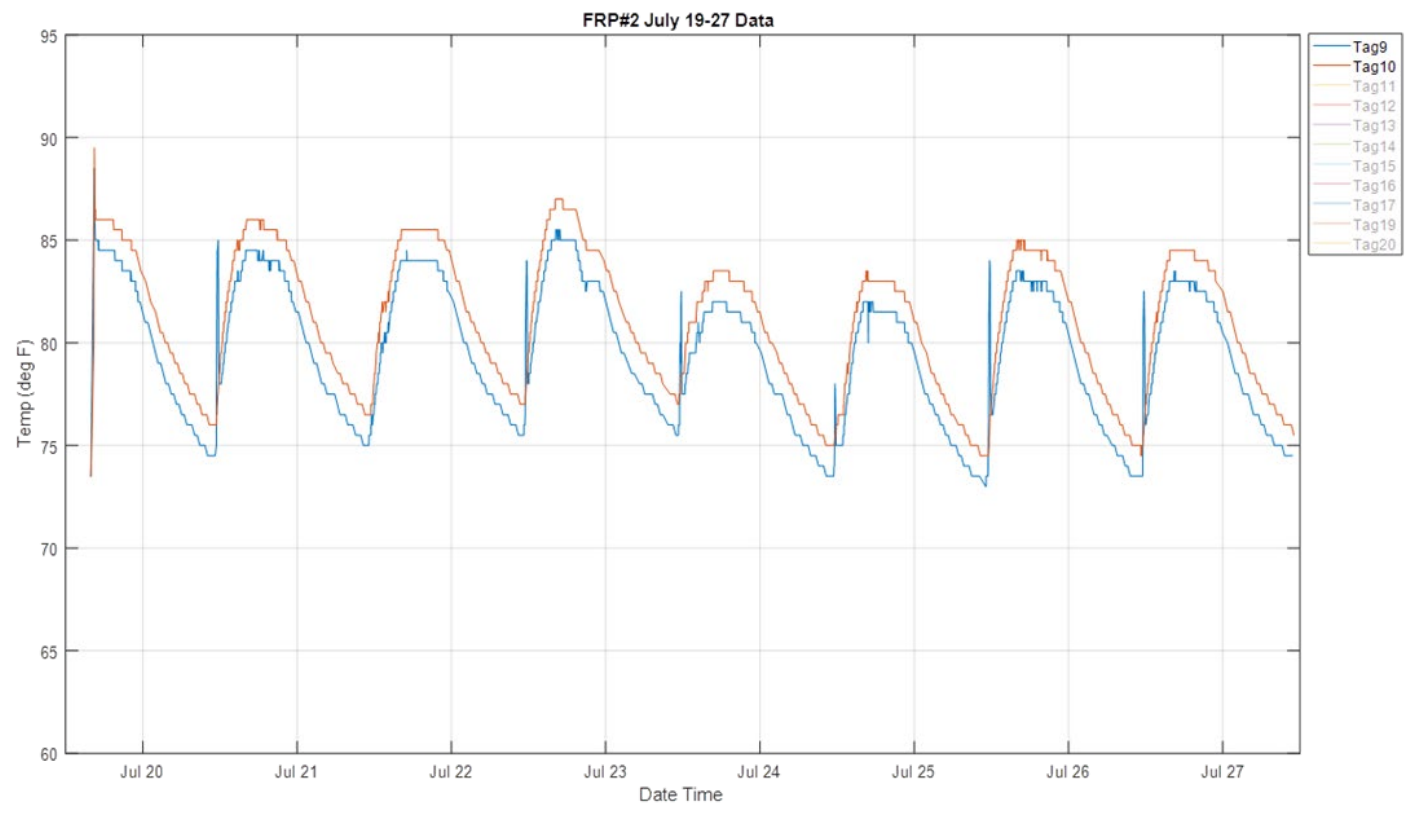

Figure 96 FRP\#2 sensor tags \#9 and \#10 (second floor) temperature measurements July 19-27.

\section{Tags \#1 - \#8 Humidity Measurements}

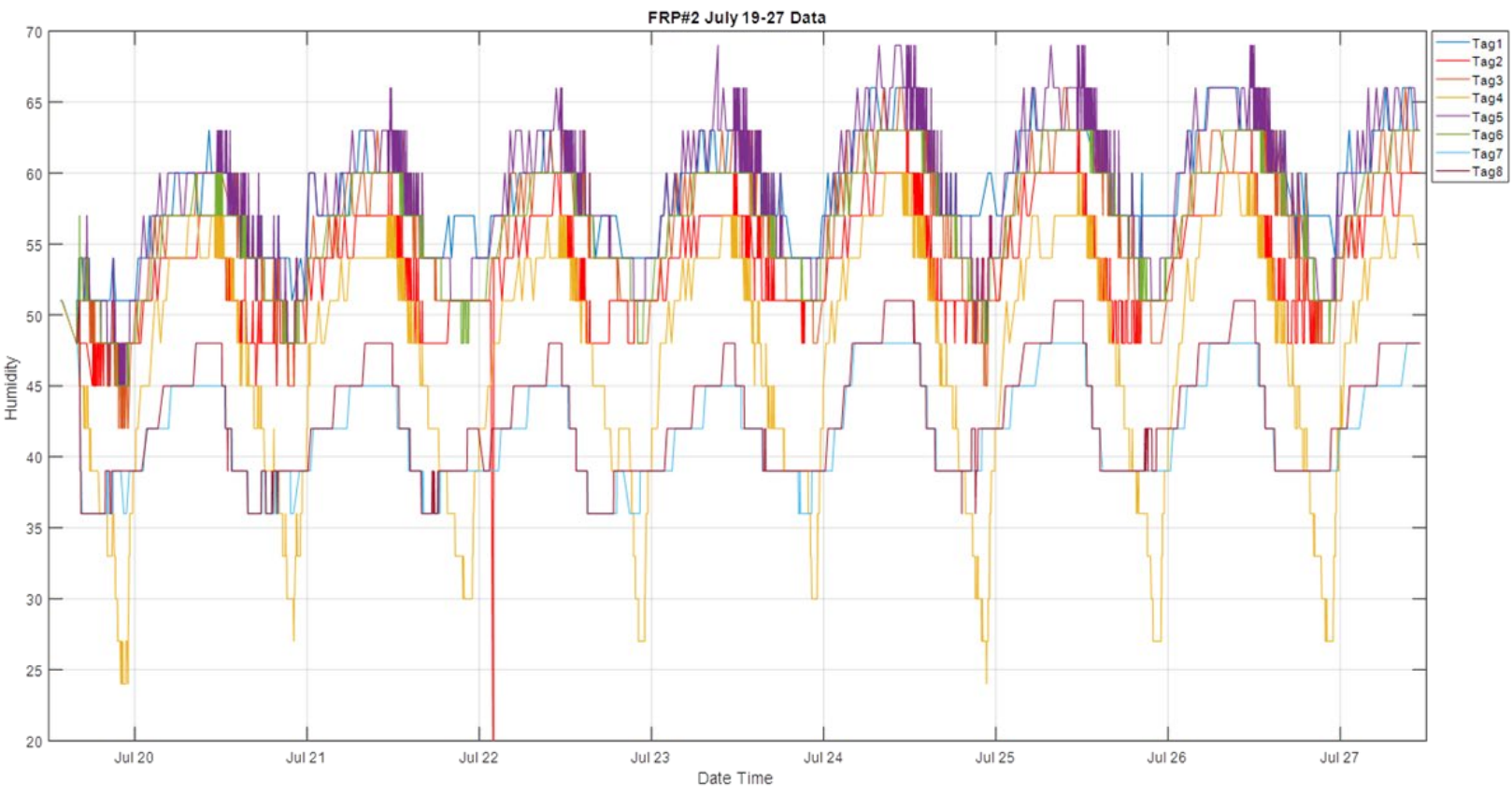

Figure 97 FRP\#2 sensor tags \#1 - \#8 (first floor) humidity measurements July 19-27. 


\section{Tags \#9-\#17, \#19-\#20 Humidity Measurements}

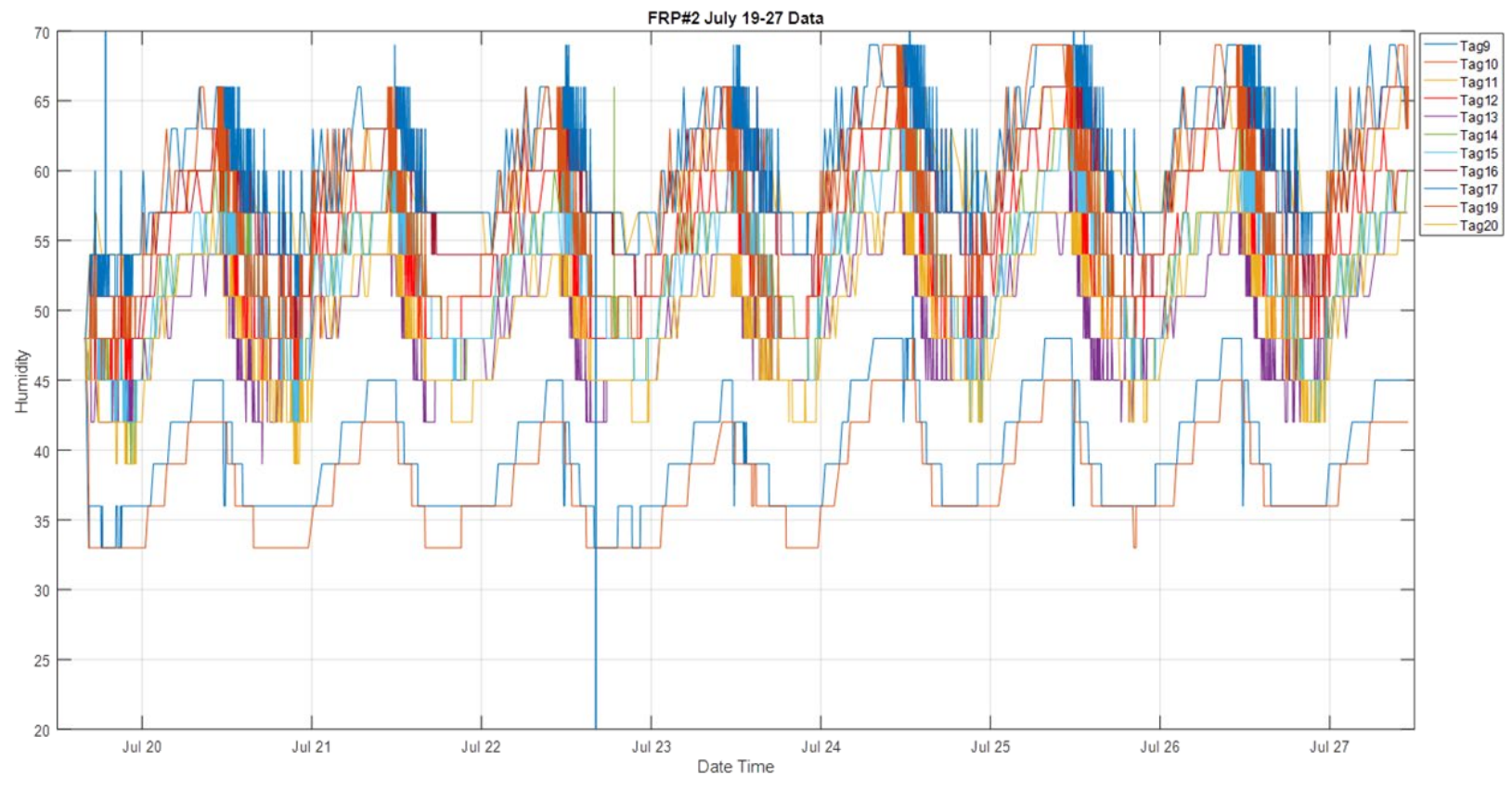

Figure 98 FRP\#2 sensor tags \#9 - \#17, \#19, and \#20 (second floor) humidity measurements July 19-27.

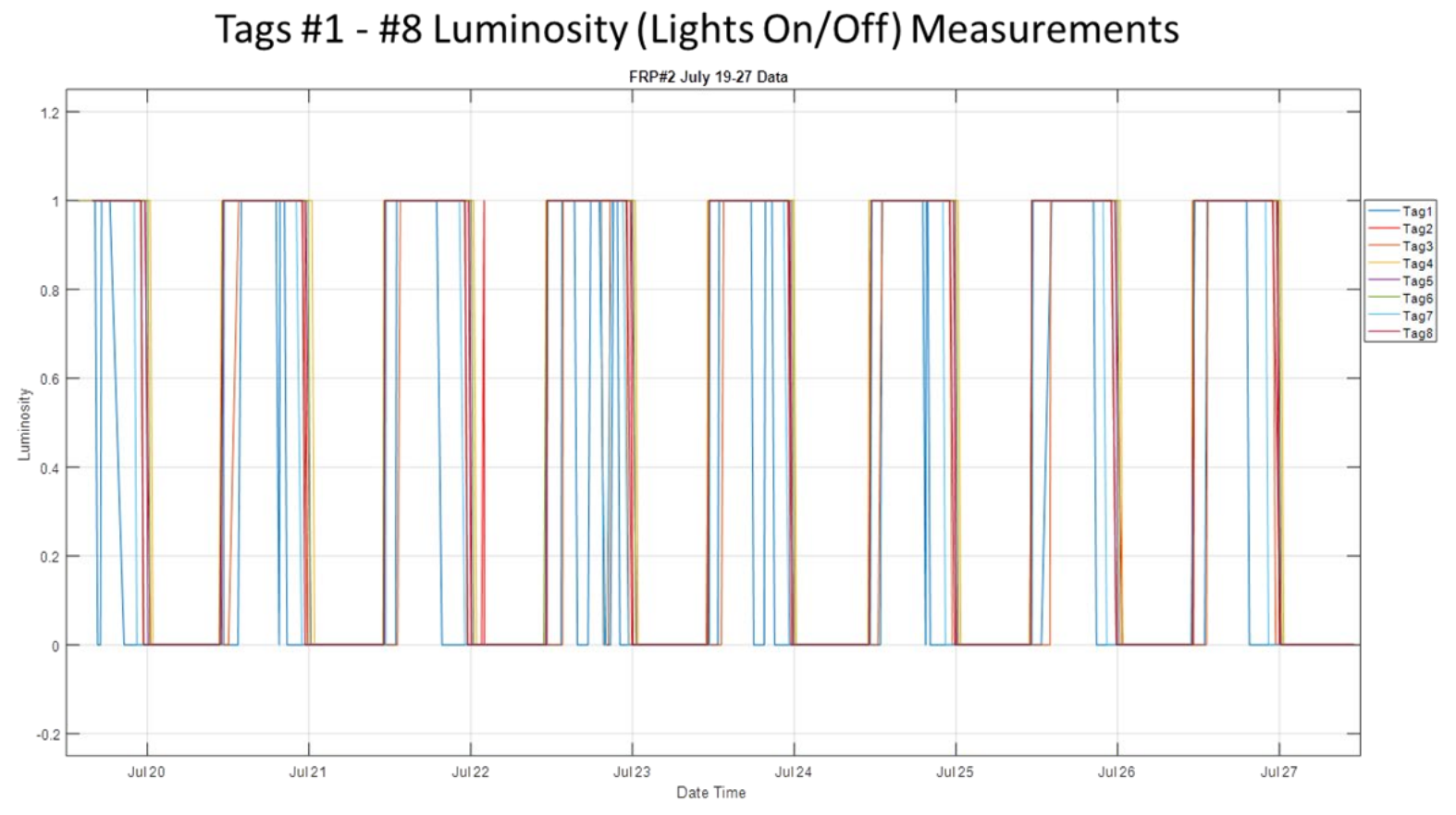

Figure 99 FRP\#2 sensor tags \#1 - \#8 (first floor) luminosity measurements July 19-27. 


\section{Tags \#9-\#17, \#19-\#20 Luminosity (Lights On/Off) Measurements}

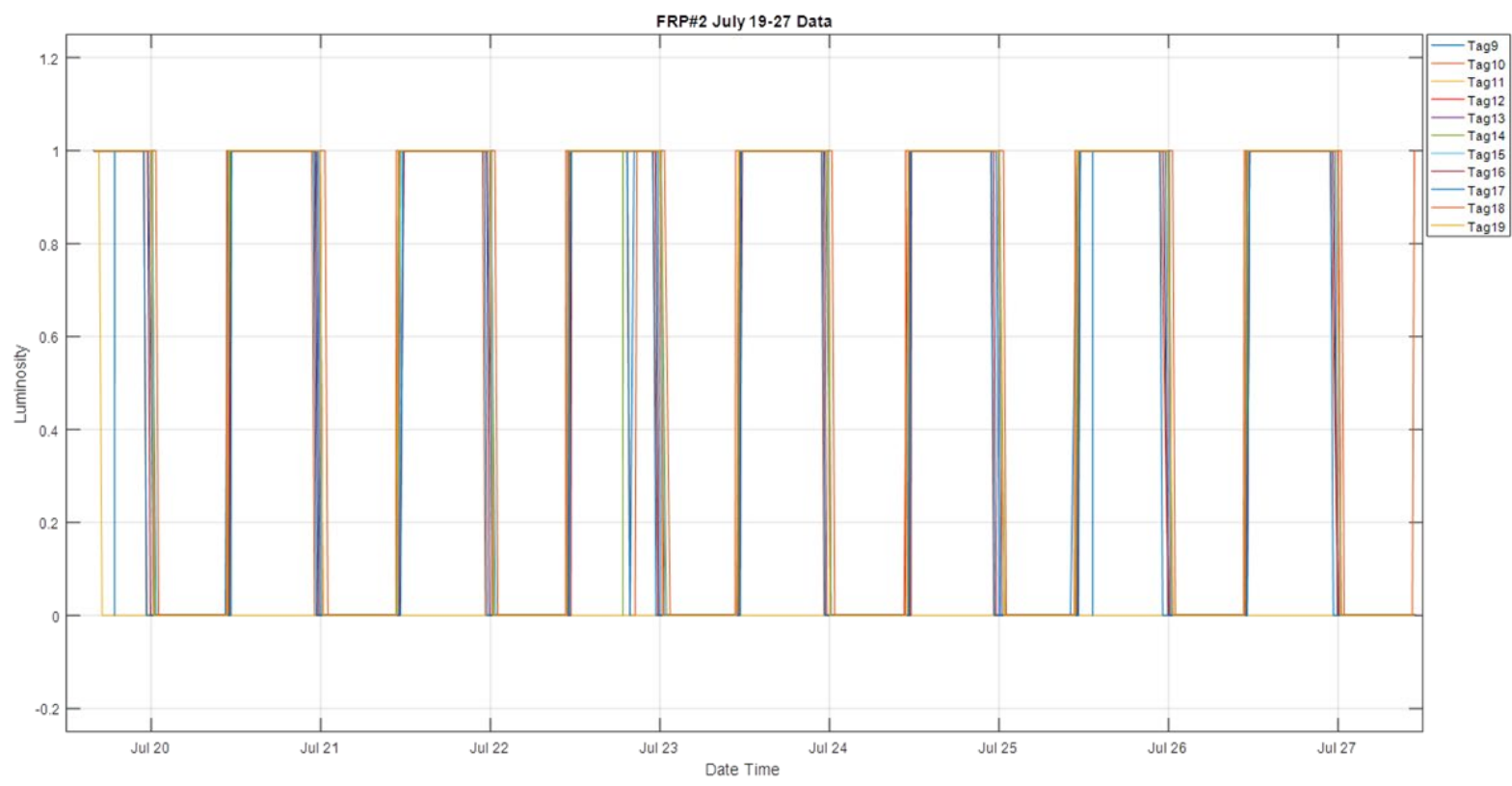

Figure 100 FRP\#2 sensor tags \#9 - \#17, \#19, and \#20 (second floor) luminosity measurements July 19-27.

Tag\# 11 Luminosity (Lights On/Off) Measurement

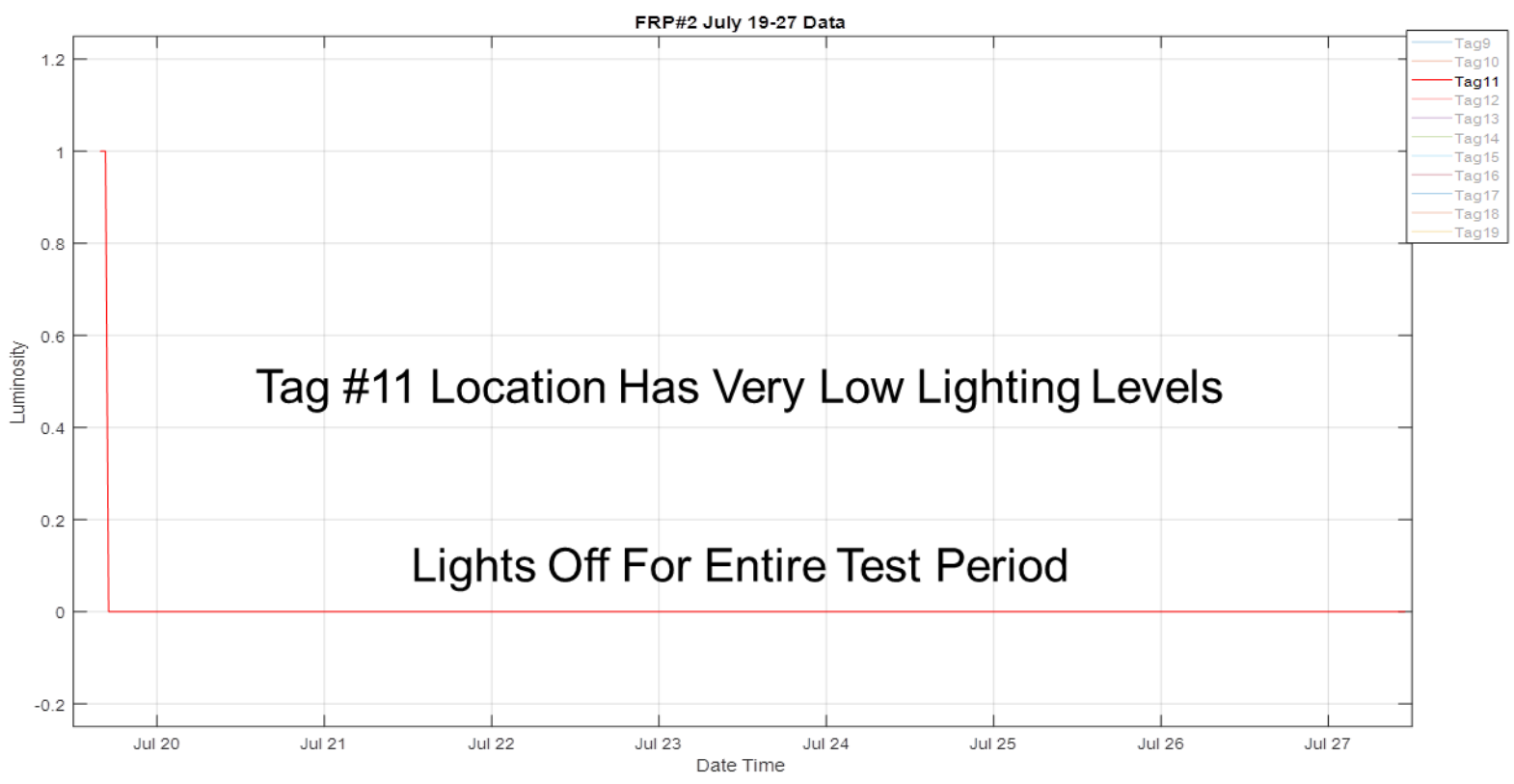

Figure 101 FRP\#2 sensor tag \#11 (second floor) luminosity measurements July 19-27. 


\section{Tag \#1 Transmit Event Rate}

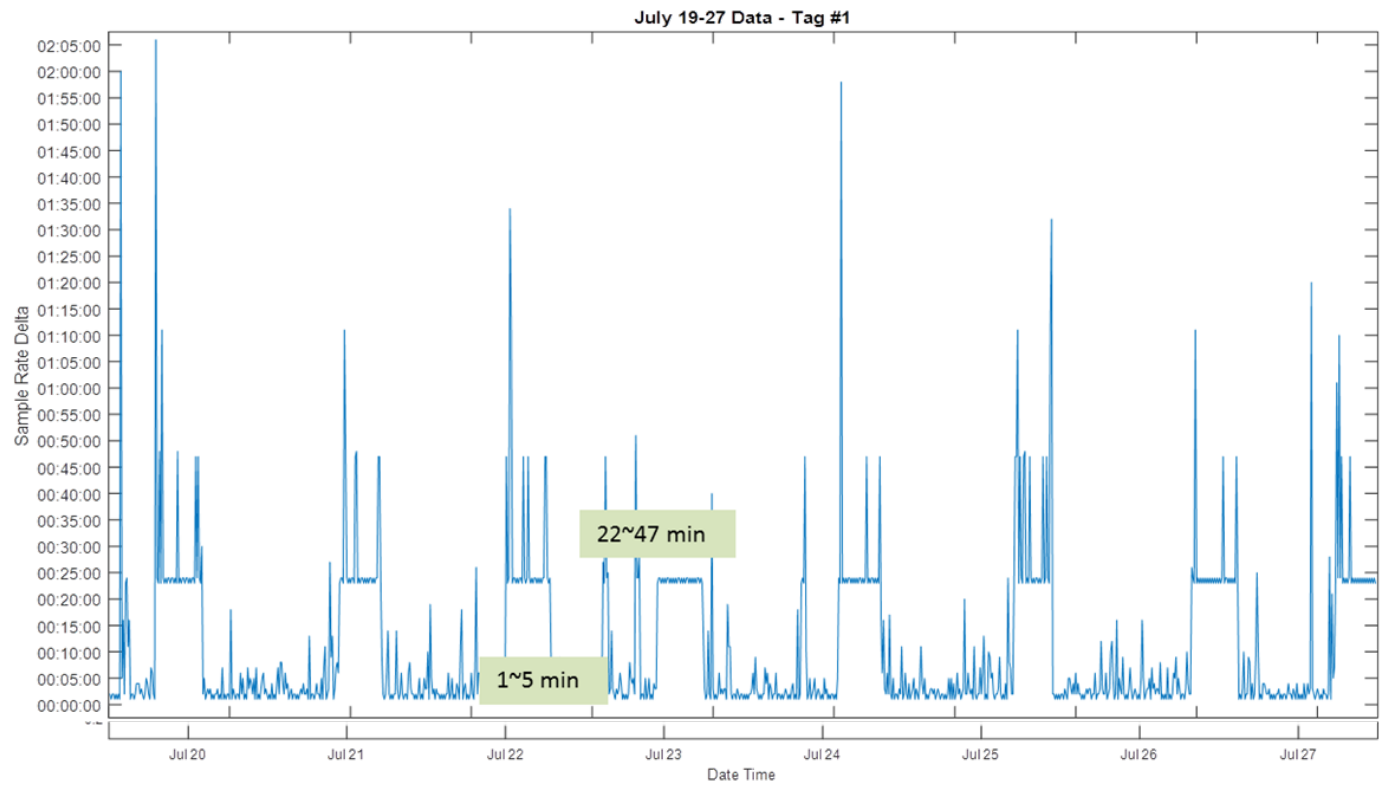

Figure 102 FRP\#2 sensor tag \#1 data sample rate from June 30 to July 19.

Tag \#2 Transmit Rate

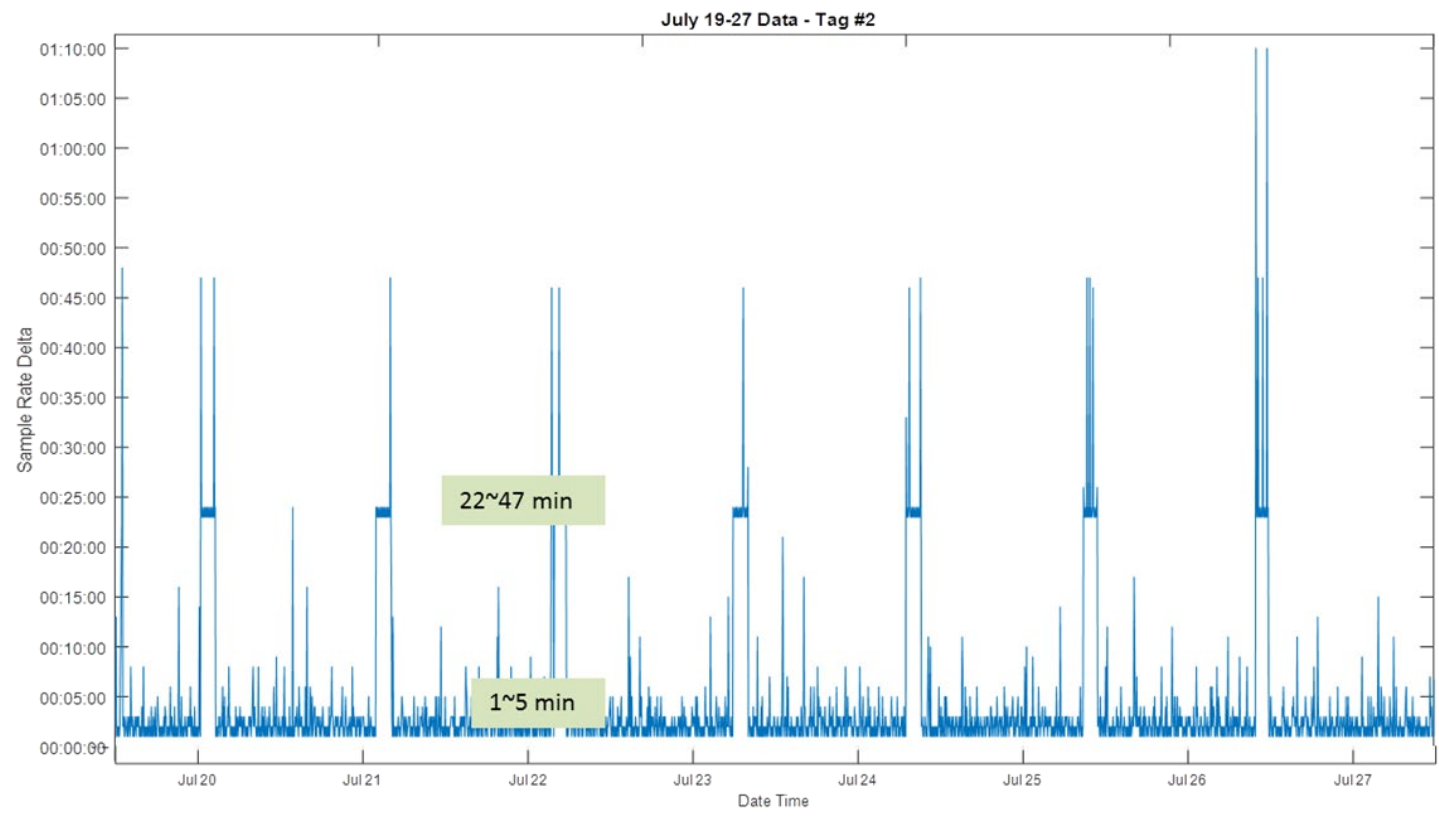

Figure 103 FRP\#2 sensor tag \#2 data sample rate from June 30 to July 19. 
Tag \#11 Data Transmit Rate

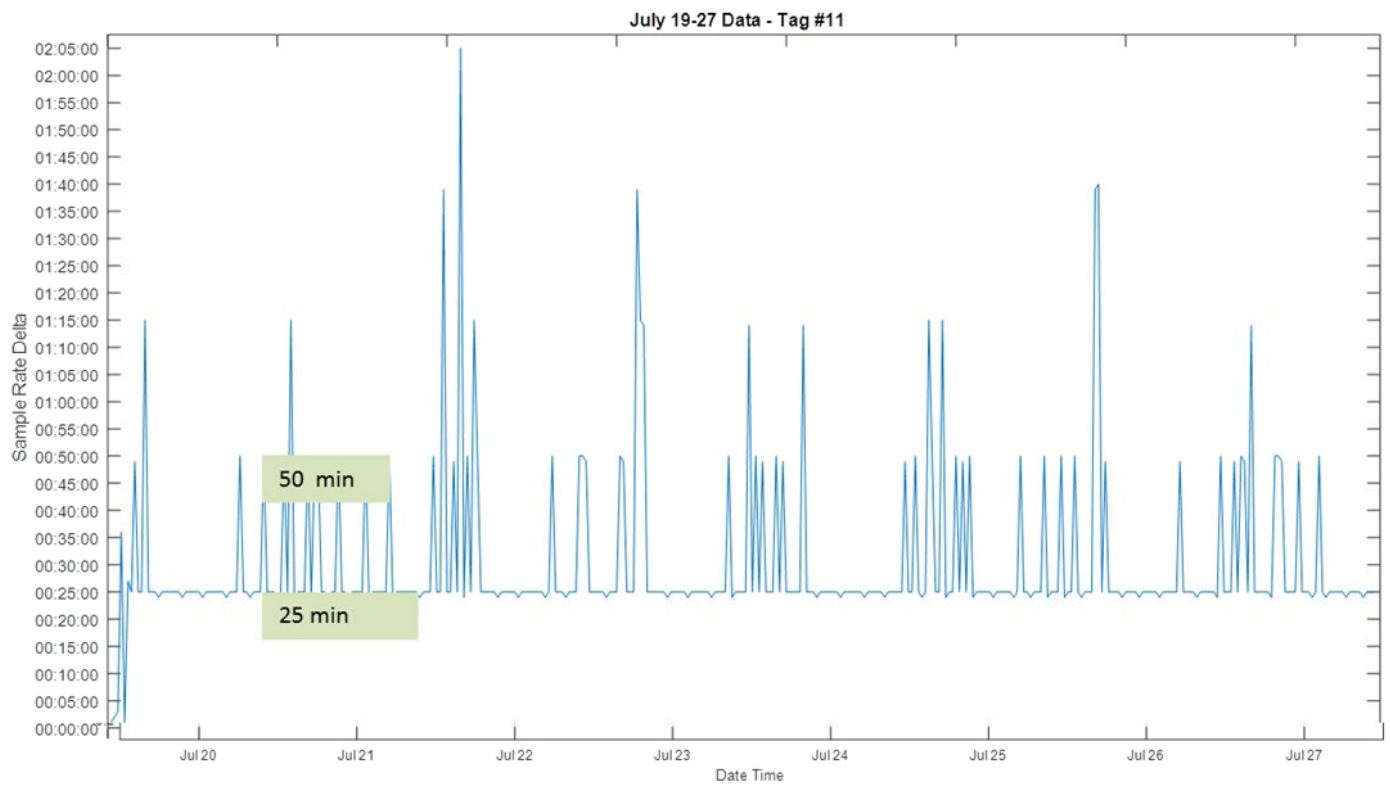

Figure 104 FRP\#2 sensor tag \#11 data sample rate from June 30 to July 19. 


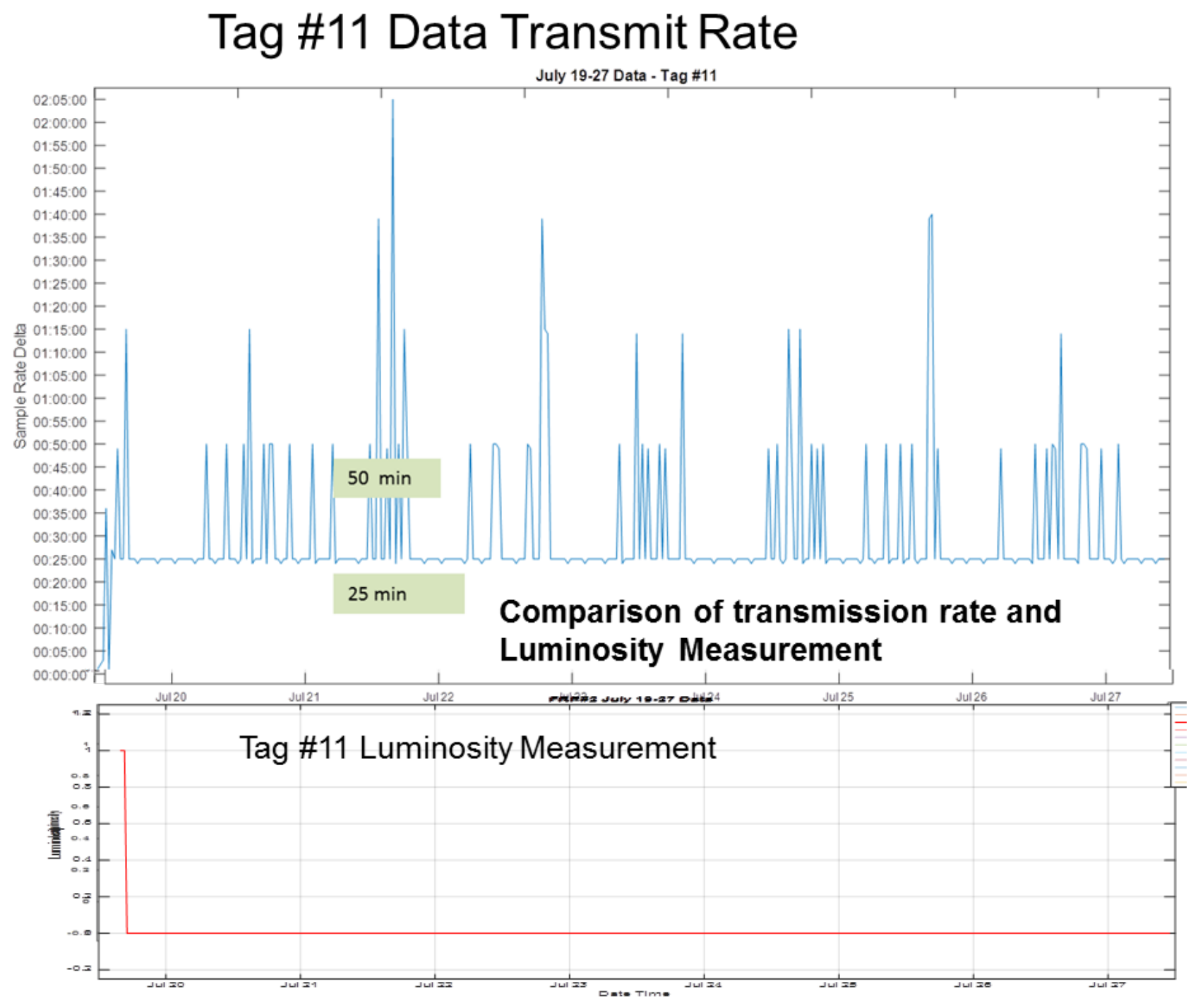

Figure 105 FRP\#2 sensor tag \#11 data sample rate from June 30 to July 19. 


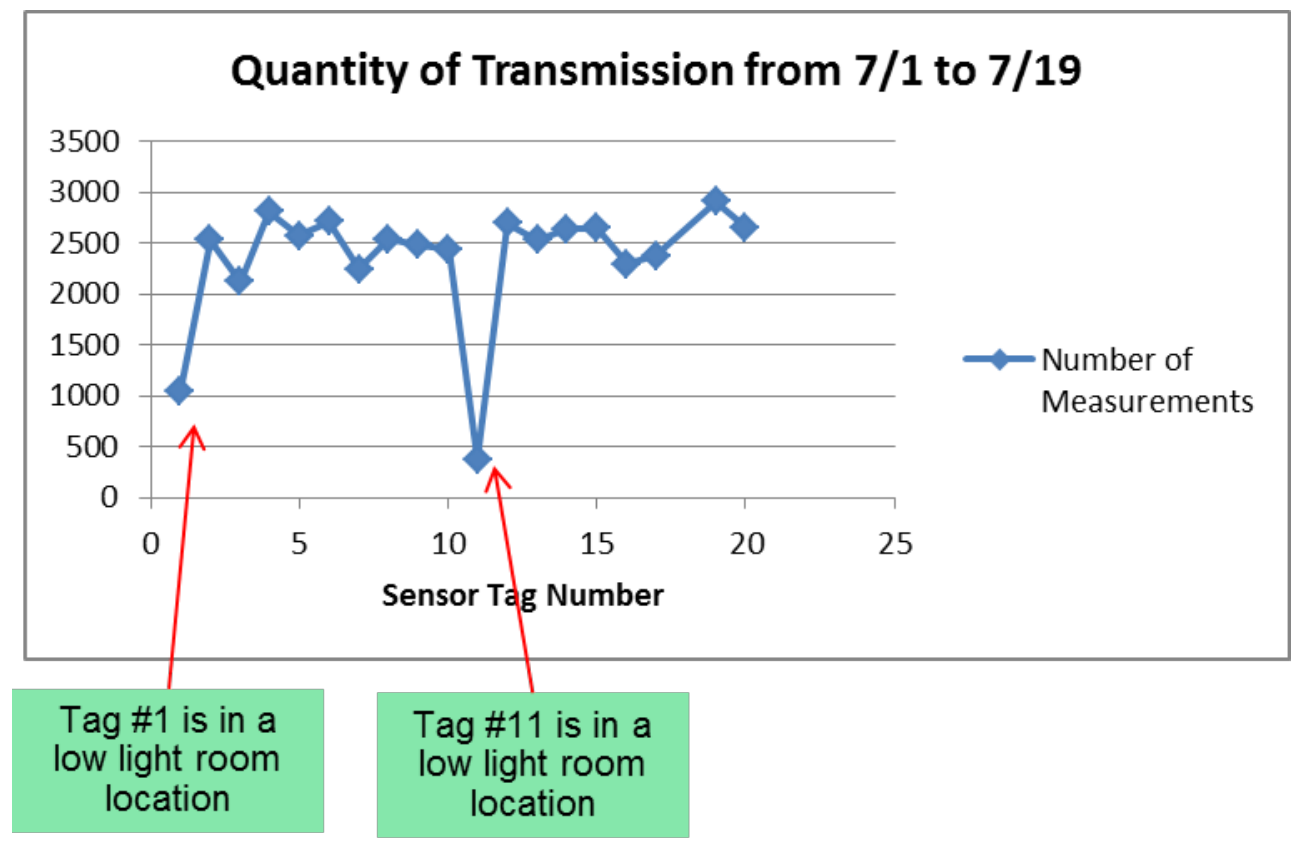

Figure 106 FRP\#2 sensor tag data transmission event analysis. 